\title{
Metabolite Profiling of a Robust Cyanobacterium for Industrial Biotechnology
}

\author{
Bethan Kultschar MChem
}

Submitted to Swansea University in fulfilment of the requirements for the Degree of Doctor of Philosophy 2020

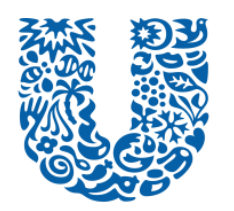

Unilever

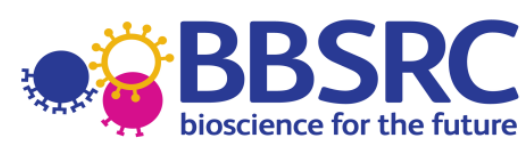





\section{Summary}

Cyanobacteria produce a variety of metabolites with diverse functions and bioactive properties that have potential uses in industrial biotechnology. Metabolomic profiles closely relate to the physiology of an organism and can be used to investigate any alteration in metabolism and production of industrially relevant metabolites. Little work has been conducted on metabolomic time-course profiles within cyanobacteria with the majority of research on targeted metabolite level changes during abiotic stress such as ultraviolet (UV) radiation. In this thesis the metabolite profile analysis of the less well investigated cyanobacterium; Chlorogloeopsis fritschii (C. fritschii) PCC 6912 was undertaken. The main aim was to evaluate changes in low molecular weight metabolite levels during standard growth and UV exposure using gas chromatographymass spectrometry (GC-MS). The focus was on identifying biologically relevant metabolites with roles in cyanobacterial metabolism associated with growth phases and adaptation to UV stress. Extracts were assessed using in vitro assays for extraction of potential anti-inflammatory and antibacterial activity from the cyanobacteria with complementary metabolomic analysis utilised for identification of potential active metabolites. Results demonstrated a significant reduction in intracellular metabolites involved in carbon and nitrogen metabolism during UV-B exposure with a higher proportion of metabolites increasing in levels during UV-A exposure. Pre-treatment of C. fritschii to low dose UV prior to high dose UV had a lesser effect on metabolism indicating stress tolerance and adaptation to UV stress. Metabolite levels were seen to reflect the changing growth phases over 120 days during standard conditions with overall similarity in metabolite levels between axenic and xenic cultures over 28 days. Finally, extracts showed promising anti-inflammatory and antibacterial activity with identification of potential active metabolites with relevance to personal care products. This is the first GC-MS based metabolite profiling of $C$. fritschii during standard growth and UV exposure. This builds on the experimental data and knowledge-base already available for cyanobacteria, including C. fritschii, for their potential use in industrial biotechnology. 


\section{Declaration and Statements}

\section{DECLARATION}

This work has not previously been accepted in substance for any degree and I not being concurrently submitted in candidature for any degree.

Signed (candidate)

Date $30 / 109 / 19$

\section{STATEMENT 1}

This thesis is the result of my own investigations, except where otherwise stated.

Other sources are acknowledged by footnotes giving explicit references. A bibliography is appended.

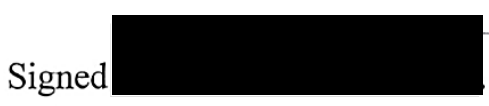
(candidate)

Date $30 / 109 / 19$

\section{STATEMENT 2}

I hereby give consent for my thesis, if accepted, to be available for photocopying and for inter-library loans after expiry of a bar on access approved by the Swansea University.

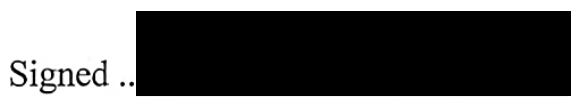
(candidate)

Date $30 / 09 / 19$ 


\section{Authorship Declaration}

The following people contributed as co-authors on a publication as part of this thesis (General Introduction):

\begin{tabular}{lll}
\hline & Name & \multicolumn{1}{c}{ Affiliation } \\
\hline Candidate & Bethan Kultschar (B.K) & Swansea University \\
Author & $\begin{array}{l}\text { Carole A. Llewellyn } \\
\text { (C.A.L) }\end{array}$ & Swansea University \\
\hline
\end{tabular}

Book chapter: Kultschar, B.; Llewellyn, C. Secondary Metabolites in Cyanobacteria. In Secondary Metabolites-Sources and Applications; InTech: London, UK, 2018; Volume 2, p.64. DOI: 10.5772/intechopen.75648

Author Contributions:

Authors contributed in the following areas: B.K was responsible for writing the manuscript with input from C.A.L. Supervision by C.A.L.

\section{Candidate Signature:}

Author Signature (C.A.L): 


\section{Authorship Declaration}

The following people contributed as co-authors on a publication as part of this thesis (Chapter 3):

\begin{tabular}{llc}
\hline & Name & Affiliation \\
\hline First Author (candidate) & Bethan Kultschar (B.K) & Swansea University \\
Author & Ed Dudley (E.D) & Swansea University \\
Author & Steve Wilson (S.W) & Unilever \\
Author & $\begin{array}{l}\text { Carole A. Llewellyn } \\
\text { (C.A.L) }\end{array}$ & Swansea University \\
\hline
\end{tabular}

Paper: Kultschar, B.; Dudley, E.; Wilson, S.; Llewellyn, C.A. Intracellular and Extracellular Metabolites from the Cyanobacterium Chlorogloeopsis fritschii, PCC 6912, During 48 Hours of UV-B Exposure. Metabolites 2019, 9, 74; DOI:10.3390/metabo9040074.

Authors contributed in the following areas: B.K was responsible for the design of the experiment, execution of experimental work and data analysis. E.D supported experimental and data acquisition. B.K wrote the manuscript with input from C.A.L, E.D and S.W. Supervision by C.A.L., E.D. and S.W.

Candidate Signature $(\square \mathrm{K})$ :

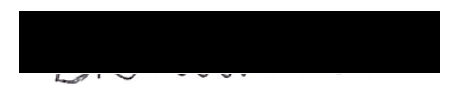

Author Signature (E.D):

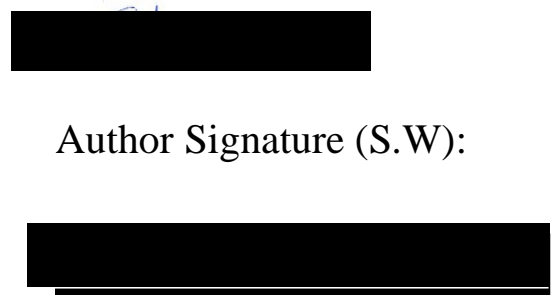

Author Signature (C.A.L): 


\section{Authorship Declaration}

The following people contributed as co-authors on a manuscript, submitted to European Journal of Phycology (in review), as part of this thesis (Chapter 3):

\begin{tabular}{llc}
\hline & Name & Affiliation \\
\hline First Author (candidate) & Bethan Kultschar (B.K) & Swansea University \\
Author & Ed Dudley (E.D) & Swansea University \\
Author & Steve Wilson (S.W) & Unilever \\
Author & $\begin{array}{l}\text { Carole A. Llewellyn } \\
\text { (C.A.L) }\end{array}$ & Swansea University \\
\hline
\end{tabular}

Manuscript: Response of key metabolites during a UV-A exposure time-series in the cyanobacterium Chlorogloeopsis fritschii, PCC 6912.

Authors contributed in the following areas: B.K was responsible for the design of the experiment, execution of experimental work and data analysis. E.D supported experimental and data acquisition. B.K wrote the manuscript with input from C.A.L, E.D and S.W. Supervision by C.A.L., E.D. and S.W.

Candidate Signature (B.K):

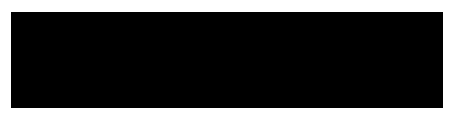

Author Signature (E.D):

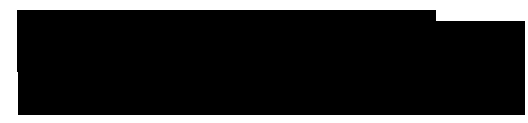

Author Signature (S.W):

Author Signature (C.A.L): 


\section{Disclaimer}

Funding: This research was funded by the Biotechnology and Biological Sciences Research Council (BBSRC iCASE studentship), UK; grant number BB/N503630/1 acquired by Prof Carole Llewellyn and Mr. Steve Skill.

Supervisors: Prof Carole A. Llewellyn, Dr Ed Dudley and Mr Steve Wilson.

The following contributions were made to the following research chapters:

Chapter 2: B.K was responsible for execution of experimental work and data analysis. E.D supported GC-MS data acquisition. Supervision by C.A.L, E.D and S.W.

Chapter 3: B.K was responsible for execution of experimental work and data analysis. E.D supported GC-MS data acquisition. Initial HPLC training was conducted by Alla Silkina and E.D. Supervision by C.A.L, E.D and S.W.

Chapter 4: B.K was responsible for execution of experimental work and data analysis. E.D supported GC-MS data acquisition. Initial conceptualisation of experiment by S.W. Supervision by C.A.L, E.D and S.W.

Chapter 5: Cultivation of cyanobacteria was carried out at SU Biosciences department and the Centre of Sustainable Aquatic Research (CSAR) with help from Claudio Fuentes Grünewald and Rob Smith. Initial bioactivity experiments were conducted at SU by B.K and Kadie Edwards (data not presented) in the school of medicine supported by Claire Morgan (mammalian cell cultivation training) and E.D. Bioactivity analysis was conducted at Unilever Research \& Development at Colworth during a 3 month placement. S.W and Mark Fowler (M.F) were responsible for design of anti-inflammatory experiments. M.F and Karen Barret supported experimental and data acquisition and data analysis. S.W and Diana Cox (D.C) were responsible for design of antibacterial experiments with D.C supporting experimental and data acquisition. 


\section{Acknowledgements}

I would firstly like to thank my supervisors for giving me the opportunity to pursue this PhD. Prof. Carole Llewellyn for the support during my time at Swansea and for giving me many opportunities within and outside of my research project, thank you Carole for everything! Dr Ed Dudley, thank you for always being on hand to source equipment, supply knowledge and expertise. I could not have done this project without you! Steve Wilson, thank you for looking after me during my 3 months at Unilever and going the extra mile with support via skype with many discussions about rugby.

Thanks to the Algae lab group espeically Alla for the advice and understanding of HPLC/PhD stress! To the PhD group at Swansea past and present, thank you to those who kept me going over the years (usually with coffee, cake and chocolate), especially Jess Ware for the last year as a great flatmate and friend. To Mark, Karen and Diana for the lab support at Unilever and sharing your knowledge with me as well as everyone else involved in my Unilever experience for making me feel so welcome! Thank you to the Unilever PhD group for always making the yearly workshops so fun, good luck for the future! Thank you to BBSRC and Unilever for funding the project.

With special thanks to my best friend Kristina Lyons for the dancing, singing and nights with prosecco in Manchester to keep me going! Finally, with the biggest thanks to my family who have always supported me in whatever I've pursued especially during the last four emotional years. My parents, Phil and Judy for always being there for me especially when I needed to come home to reset and recharge. Thanks for always being on the end of the phone, full of wise words and wisdom to get me through the day! My amazing brothers, Tom and Mike thank you for always checking up on me and giving the best advice! Thanks to Tom, Mike and Lozzie for letting me escape to London (London club). I can't wait to join you up there in November Mike! Tom, Lozzie and Duglas (woof) for now letting me escape to Caerphilly with nights full of wine (Dino) and puppy cwtches! Diolch o'r galon, I couldn't have done it without you! 
"Take a deep breath, all things will pass"

This thesis is dedicated to my family;

Phil, Judy, Tom, Mike and my Gran (Elvira)

In loving memory of my grandparents;

Bram and Betty 


\section{Contents}

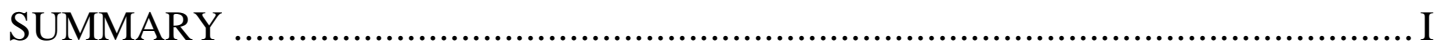

DECLARATION AND STATEMENTS...............................................................

AUTHORSHIP DECLARATION..................................................................II

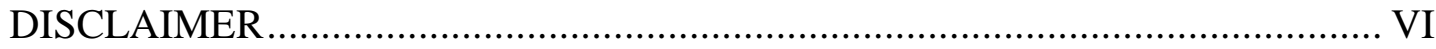

ACKNOWLEDGEMENTS …………………………………………....... VII

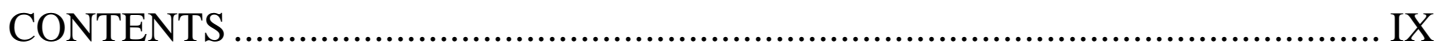

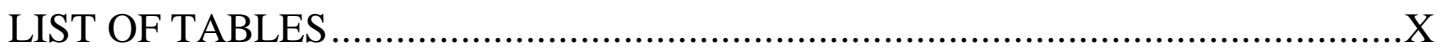

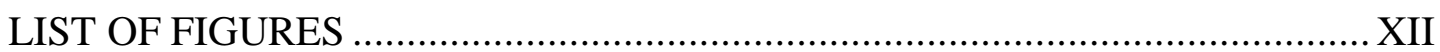

LIST OF EQUATIONS .....................................................................................

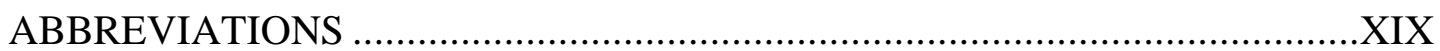

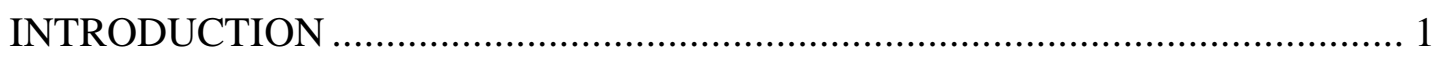

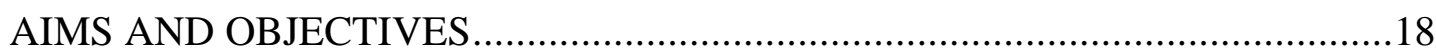

CHAPTER 1: MATERIALS AND METHODS ……………………………….....

CHAPTER 2: TIME-SERIES METABOLITE LEVEL INVESTIGATION OF CHLOROGLOEOPSIS FRITSCHII, PCC 6912, DURING STANDARD GROWTH CONDITIONS.

CHAPTER 3: INTRACELLULAR AND EXTRACELLULAR METABOLITE LEVEL CHANGES OF CHLOROGLOEOPSIS FRITSCHII, PCC 6912, DURING ULTRAVIOLET (UV) A AND UV-B EXPOSURE

CHAPTER 4: ACCLIMATION VS. SHOCK: THE STRESS RESPONSE OF CHLOROGLOEOPSIS FRITSCHII, PCC 6912, TO UV EXPOSURE

CHAPTER 5: BIOACTIVITY ASSESSMENT OF CHLOROGLOEOPSIS FRITSCHII, PCC 6912 IN IN-VITRO ANTI-INFALMMATORY AND

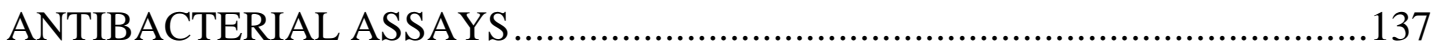

CHAPTER 6: GENERAL DISCUSSION ……………………........................169

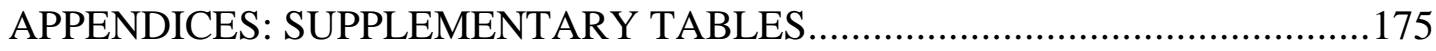

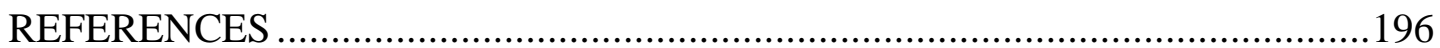




\section{List of Tables}

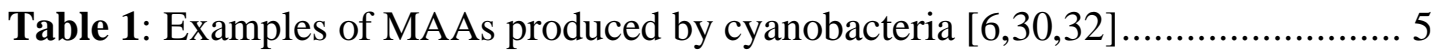

Table 2: Untargeted and targeted metabolite extraction methods in varying cyanobacterial species.

Table 3: Potential applications of cyanobacterial natural products in pharmaceuticals and beauty personal care products $[6,17]$.

Table 4: High-performance liquid chromatography (HPLC) gradient protocol for mycosporine-like amino acid (MAA) separation used throughout method development. .25

Table 5: Dilution series for BSA standard curve.................................................30

Table 6: Dilution series for glucose standard....................................................... 32

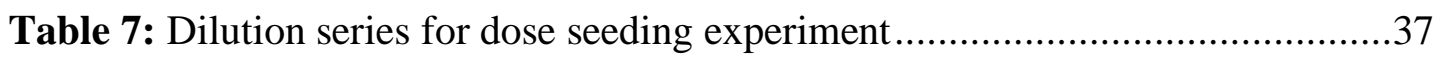

Table 8: Dilution series for TNF- $\alpha$ and IL-1 $\beta$ dose response ................................39

Table 9: Extended dilution series for TNF- $\alpha$ dose response .................................40

Table 10: Dilution series for HC anti-inflammatory dose response .......................41

Table 11: Dilution series for dose response of $C$. fritschii extracts ........................42

Table 12: Dilution series for m-gly anti-inflammatory dose response. ...................43

Table 13: Preparation of IL-6 and IL- 8 reagents. IL- 6 reagent diluent $=1 \%$ BSA in PBS, IL- 8 reagent diluent $=0.1 \%$ BSA, $0.05 \%$ TWEEN® 20 in Tris-Buffered Saline.

Table 14: BCA standard preparation for protein assay

Table 15: Combination of disruption techniques and solvents for extraction of metabolites from $C$. fritschii.

Table 16: Comparison of significantly different $(p<0.05)$ peaks (out of 564) and number of those significantly different peaks with a positive or negative $\log 2 \mathrm{FC}$ within axenic cultures.

Table 17: Comparison of significantly different $(p<0.05)$ peaks (out of 535) and number of those significantly different peaks with a positive or negative $\log 2 \mathrm{FC}$ within xenic cultures.

Table 18: Number of significantly different $(p<0.05)$ biologically relevant metabolite levels from axenic and xenic cultures including comparison between the two

Table 19: Comparison of significantly different $(p<0.05)$ peaks (out of 137) and number of those significantly different peaks with a positive or negative $\log 2 \mathrm{FC}$ during PAR only exposure

Table 20: Resolution factor (Rs) between detected peaks separated using different RPcolumns

Table 21: Retention time (RT), relative standard deviation (RSD \%) and UVabsorbance maxima ( $\lambda \max )$ for each peak detected using different RP-columns......91 
Table 22: Peak identifications including retention time (RT), relative standard deviation (RSD \%), resolution factor (Rs) between peaks and UV-absorbance maxima $(\lambda \max )$.

Table 23: Comparison of statistically significant $(p<0.05)$ peaks (out of 175) and number of those significant peaks with a positive or negative $\log 2 \mathrm{FC}$. 106

Table 24: Comparison of statistically significant $(p<0.05)$ peaks and number of those significant peaks with a positive or negative $\log 2 \mathrm{FC}$ during supplemented UV-B exposure 108

Table 25: PAR, UV-B and UV-A intensities during acclimation and shock experiments. PAR + UV-B treatments were carried out separately from PAR + UV-A. 120

Table 26: Identified metabolites in control $C$. fritschii extracts (hex, EtOAc, EtOH and $\mathrm{MeOH}$ ) including chemical class, KEGG pathway and \% normalised abundance across all 4 extracts. 162

Table 27: Metabolites identified in $C$. fritschii extracts with potential activity with relevance to beauty personal care. 165

\section{List of Supplementary Tables}

Table S1: Identified intracellular metabolites detected during $28 \mathrm{~d}$ experiment (axenic) including chemical class and possible biosynthetic pathways (KEGG)...175

Table S2: Identified intracellular metabolites detected during $28 \mathrm{~d}$ experiment (xenic) including chemical class and possible biosynthetic pathways (KEGG).

Table S3: Identified intracellular metabolites detected during 120 d experiment including chemical class and possible biosynthetic pathways (KEGG)

Table S4: Identified intracellular metabolites detected during $48 \mathrm{~h}$ of PAR only including chemical class and possible biosynthetic pathways (KEGG). 184

Table S5: Identified intracellular and extracellular metabolites detected during UV-B + PAR including chemical class and possible biosynthetic pathways (KEGG)......186

Table S6: Identified intracellular metabolites detected during UV-A + PAR including chemical class and possible biosynthetic pathways (KEGG).

Table S7: Identified intracellular metabolites detected during acclimation \& shock and shock only UV-A + PAR experiments including chemical class and possible biosynthetic pathways (KEGG). 191

Table S8: One-way ANOVA results showing metabolites with overall significance ( $p$ $<0.05$ ) including Tukey's Honestly Significant difference (HSD) post-hoc test results of significant pairs of solvent extracts. 195 


\section{List of Figures}

Figure 1: Effect of Ultraviolet (UV) radiation on cyanobacteria .......................... 4

Figure 2: Biosynthetic pathways for the production of mycosporine-like amino acids (MAAs) via 1) Shikimate pathway and 2) pentose phosphate pathway via the common intermediate 4-deoxygadusol (4-DG) [29].

Figure 3: Final metabolite profiling workflow using GC-MS

Figure 4: Generalised biosynthetic pathway schematic of key metabolite production from $\mathrm{CO}_{2}$ assimilation in cyanobacteria.

Figure 5: Lowry assay standard curve using BSA. Straight line equation: $\mathrm{y}=0.6981 \mathrm{x}$ $+0.0027, R^{2}=0.9975$.

Figure 6: Phenol-sulfuric acid assay standard curve using glucose. Straight line equation: $\mathrm{y}=1.9516 \mathrm{x}-0.0102, \mathrm{R}^{2}=0.9987$.

Figure 7: Two-fold dilution series of IL-6 and IL-8 standards.

Figure 8: Principle component analysis (PCA) of metabolites extracted using different techniques and solvents.

Figure 9: ANOVA-Simultaneous Component Analysis (ASCA) scree plots showing major patterns associated with A) technique and B) solvent generated from total peaks detected across all samples.

Figure 10: Hierarchial heatmap visualisation of the significanty different peaks $(p<0.05)$, using identified peaks only, comparing each extraction protocol using a twoway between samples ANOVA in MetaboAnalyst.

Figure 11: Total number of peaks detected across all extraction protocols including number of identified and unassigned peaks.

Figure 12: Number of identified peaks extracted from $C$. fritschii cells by chemical class for each extraction protocol.

Figure 13: Dry weight of axenic and xenic $C$. fritschii cultures $(\mathrm{n}=3)$ during growth over 28 days under control conditions sampling at $0,4,8,14,20$ and 28 days. Each data point is the mean value of biological triplicates \pm standard error $(\mathrm{SE})$.

.58

Figure 14: Specific growth rate $(\mu)$ of axenic and xenic $C$. fritschii cultures between time points showing mean values \pm SE.

Figure 15: (A) Bulk carbohydrate content of axenic and xenic $C$. fritschii cultures during 28 days of growth. (B) Bulk protein content of axenic and xenic C. fritschii cultures during 28 days of growth.

Figure 16: PCA of detected peaks over 28 days from A) axenic and B) xenic $C$. fritschii cultures. Each ring represents distribution of replicates from each time points.

Figure 17: Overview of biologically relevant metabolites detected in axenic cultures over 28 days. A) Overall \% composition of detected metabolites by chemical class. B, C, D and E) Identified metabolites by class as acids (involved in TCA), amino acids, fatty acids and sugars (and derivatives) over the whole time-series.

Figure 18: Overview of biologically relevant metabolites detected in xenic cultures over 28 days. A) Overall \% composition of detected metabolites by chemical class. B, 
C, D and E) Identified metabolites by class as acids (involved in TCA), amino acids, fatty acids and sugars (and derivatives) over the whole time-series.

Figure 19: Dry weight of $C$. fritschii cultures (n=3) during growth over 120 days under control conditions sampling every 8 days between 0 and 120 days, day 32 data omitted. Each data point is the mean value of biological triplicates \pm SE.

Figure 20: Dry weight of individual $C$. fritschii cultures during growth over 120 days under control conditions sampling every 8 days between 0 and 120 days, day 32 data omitted.

Figure 21: PCA of identified peaks (367) over 120 days from $C$. fritschii cultures. Samples from day 48 (S3) and 104 (S3) have been omitted.. .68

Figure 22: Overview of biologically relevant metabolites detected over the 120 days. A) Overall \% composition of detected metabolites by chemical class. B) Overall \% of metabolites identified as acids by associated pathway. C, D, E and F) Identified metabolites by class as acids (involved in TCA), amino acids, fatty acids and sugars (and derivatives) over the whole time-series.

Figure 23: Composition of identified biologically relevant metabolites over time by chemical class.

Figure 24: Metabolites involved in TCA cycle identified using GC-MS presented as mean values of normalised abundance \pm SE.

Figure 25: Metabolites involved in amino acid biosynthesis identified using GC-MS presented as mean values of normalised abundance \pm SE.

Figure 26: Metabolites involved in fatty acid biosynthesis identified using GC-MS presented as mean values of normalised abundance \pm SE. . .

Figure 27: Metabolites involved in sugar biosynthesis identified using GC-MS presented as mean values of normalised abundance \pm SE. .

Figure 28: Dry weight of $C$. fritschii cultures $(\mathrm{n}=3)$ during growth over $48 \mathrm{~h}$ under control conditions sampling at 0,2, 6, 12, 24 and $48 \mathrm{~h}$. .74

Figure 29: Schematic representation of a generalised reduced carbon metabolism in the cyanobacterium $C$. fritschii showing glycolysis, TCA cycle, amino acid and fatty acid biosynthesis .76

Figure 30: Metabolites involved in amino acid biosynthesis identified using GC-MS presented as mean values of normalised abundance \pm SE. .77

Figure 31: Metabolites involved in sugar biosynthesis identified using GC-MS presented as mean values of normalised abundance \pm SE.

Figure 32: Metabolites involved in the TCA cycle identified using GC-MS presented as mean values of normalised abundance \pm SE.

Figure 33: Metabolites involved in fatty acid biosynthesis identified using GC-MS presented as mean values of normalised abundance \pm SE.

Figure 34: A) Resolution factor (Rs) between peaks detected separated using the three different compositions of eluent A and B) Relative standard deviation (RSD \%) of the detected peak areas for the three compositions of eluent $\mathrm{A}$.

Figure 35: HPLC chromatograms of the MAA profile of crude extract 1 monitored at $330 \mathrm{~nm}$ using different columns: A) Alltima ${ }^{\circledR}$ Altech RP C18 column $(5 \mu \mathrm{m}, 4.6 \mathrm{x}$ 
$150 \mathrm{~mm})$; B) Synergi ${ }^{\mathrm{TM}}$ Fusion RP C18 column ( $\left.4 \mu \mathrm{m}, 4.6 \times 150 \mathrm{~mm}, 80 \AA\right)$; C) Hichrom RP ACE excel super-C18 column ( $3 \mu \mathrm{m}, 4.6$ x $250 \mathrm{~mm}, 80 \AA$ A); D) Hichrom RP ACE excel C18- Amide column ( $3 \mu \mathrm{m}, 4.6$ x $250 \mathrm{~mm}, 100 \AA)$, E) Hypercarb® RP C30 column ( $5 \mu \mathrm{m}, 4.6 \times 100 \mathrm{~mm}, 250 \AA)$ and F) Develosil@ C30-UG column (5 $\mu \mathrm{m}$, 4.6 x $250 \mathrm{~mm}, 140 \AA)$. .90

Figure 36: Optimisation protocol showing the different concentrations of TFA in eluent A followed by the different columns used. . .92

Figure 37: UV-visible spectra (295 - $455 \mathrm{~nm})$ of crude extract 1 (+ usujirene), crude extract 2 (- usujirene) and crude extract 3 (Shinorine \& Porphyra-334).

Figure 38: HPLC chromatograms of the MAA profiles of; A) crude extract 1 at 330 $\mathrm{nm}$; B) crude extract 1 at $360 \mathrm{~nm}$; C) crude extract 2 at $330 \mathrm{~nm}$ and D) crude extract 3 at $330 \mathrm{~nm}$.

Figure 39: HPLC chromatograms of the MAA profile of the crude $P P$ extract showing absorbance at $330 \mathrm{~nm}$ (top) and $360 \mathrm{~nm}$ (bottom): 1) Shinorine, $\lambda \max =332 \mathrm{~nm} ; 2$ ) Palythine, $\lambda \max =320 ; 3)$ Asterina, $\lambda \max =332 \mathrm{~nm} ; 4$ ) Porphyra-334, $\lambda \max =334 ; 5$ ) Usujirene, $\lambda \max =356 \mathrm{~nm}$ and 6) Palythene, $\lambda \max =358 \mathrm{~nm}$.

Figure 40: Dry weight measurements of $\mathrm{C}$. fritschii cultures during $48 \mathrm{~h}$ of supplemented UV-B exposure. All values are the mean of three biological replicates \pm SE. .96

Figure 41: Carotenoid and mycosporine-like amino acid (MAA) analysis of C. fritschii extracts during UV-B exposure. A) Total carotenoid concentration as measured by UV-visible spectroscopy and B) shinorine and mycosporine-glycine (mgly) content measured by HPLC analysis.

Figure 42: Principle component analysis (PCA) of A) intracellular and $\mathrm{B}$ ) extracellular GC-MS data of UV-B exposed (PAR + UV-B) C. fritschii cultures showing PC1 vs PC2 only. Each ring represents distribution of biological replicates.

Figure 43: Hierarchical heatmap visualisation of the significant $A$ ) intracellular and B) extracellular peak intensities ( $p<0.05)$ during UV-B exposure using a one-way repeated measure ANOVA in MetaboAnalyst..

Figure 44: Schematic representation of a generalised reduced carbon metabolism in the cyanobacterium $\mathrm{C}$. fritschii showing glycolysis, the TCA cycle, amino acid and fatty acid biosynthesis. Primary metabolites identified in intracellular samples using GC-MS are highlighted in blue with each insert presenting mean values of normalised abundance (normalised to internal standard and dry weight) \pm SE of each metabolite during supplemented UV-B exposure (PAR + UV-B).Error! Bookmark not defined. 1

Figure 45: Time-series extracellular GC-MS data of $C$. fritschii over $48 \mathrm{~h}$ of UV-B exposure showing primary metabolites found in extracellular samples only.

Figure 46: Time-series GC-MS data of $C$. fritschii during $48 \mathrm{~h}$ of UV-B exposure showing primary metabolites found in both intra- and extracellular samples. A) Metabolites showing significantly different changes $(p<0.05)$ over time in both intraand extracellular data; B) metabolites showing significantly different changes $(p<0.05)$ over time in intracellular samples only. 104 
Figure 47: A) Dry weight (mg L-1) and B) carotenoid content of $C$. fritschii cultures $(\mathrm{n}=3)$ during supplemented UV-A exposure over $48 \mathrm{~h}$.

1075

Figure 48: Schematic representation of a generalised reduced carbon metabolism in the cyanobacterium C. fritschii. Ten primary metabolites were identified in intracellular samples using GC-MS and are highlighted in purple with each graph presenting mean values of normalised abundance (normalised to internal standard and dry weight) \pm standard error of each metabolite during supplemented UV-A exposure (UV-A + PAR) Error! Bookmark not defined.7

Figure 49: Total number of statistically significant peaks $(p<0.05)$ with positive and negative $\log 2$ Fold Change (FC) comparing control $(0 \mathrm{~h})$ to each time point 2, 6, 12 , 24 and $48 \mathrm{~h}$ of UV exposed (UV-A + PAR or UV-B + PAR) and PAR only experiments.

Figure 50: $\log 2 \mathrm{FC}$ of commonly identified metabolites using GC-MS analysis during supplemented UV-A (purple), supplemented UV-B (blue) and PAR only (orange) exposure comparing $0 \mathrm{~h}$ and each time point $(2,6,12,24$ and $48 \mathrm{~h}$ ). 110

Figure 51: $\log 2 \mathrm{FC}$ of commonly identified metabolites using GC-MS analysis during supplemented UV-A (purple) and PAR only (orange) exposure comparing $0 \mathrm{~h}$ and each time point $(2,6,12,24$ and $48 \mathrm{~h})$....

Figure 52: $\log 2 \mathrm{FC}$ of commonly identified metabolites using GC-MS analysis during supplemented UV-A (purple) and UV-B (blue) exposure comparing no UV ( $0 \mathrm{~h})$ and each time point $(2,6,12,24$ and $48 \mathrm{~h})$

Figure 53: Dry weight analysis $\left(\mathrm{mg} \mathrm{L}^{-1}\right)$ of $C$. fritschii cultures during A) UV-B acclimation and shock and B) UV-B shock only. Data points are mean values of biological triplicates \pm SE.

Figure 54: Growth rate $(\mu)$ of UV-B acclimation and shock vs. shock only of $C$. fritschii cultures. Low UV-B and no UV-B accounts for the growth rates between 0 and $24 \mathrm{~h}$ and high UV-B accounts for growth rate between 24 and $48 \mathrm{~h}$. Data represents mean growth rates of biological triplicates \pm SE.

Figure 55: Pigment content ( $\mu \mathrm{g} / \mathrm{mg}$ dry weight) of $C$. fritschii cultures during A) UV$B$ acclimation and shock and B) UV-B shock only. Data points are mean values of biological triplicates \pm SE.

Figure 56: MAA content ( $\mu \mathrm{g} / \mathrm{mg}$ dry weight) of $C$. fritschii cultures during A) UV-B acclimation and shock and B) UV-B shock only. Data points are mean values \pm SE.

Figure 57: Number of detected peaks that show significant differences $(p<0.05)$ during acclimation and shock (total peaks $=428)$ and shock only (total peaks $=396)$ comparing 0 vs. $24 \mathrm{~h}$ (low UV-B or no UV-B) and 24 vs. $48 \mathrm{~h}$ (high UV-B) using a two-sample t-Test with equal variance..

125

Figure 58: Dry weight analysis $\left(\mathrm{mg} \mathrm{L}^{-1}\right)$ of $C$. fritschii cultures during A) UV-A acclimation and shock and B) UV-A shock only. Data points are mean values of biological triplicates \pm SE.

Figure 59: Growth rate $(\mu)$ of UV-A acclimation and shock vs. shock only of $C$. fritschii cultures. Low UV-A and no UV-A accounts for the growth rates between 0 and $24 \mathrm{~h}$ and high UV-A accounts for growth rate between 24 and $48 \mathrm{~h}$. Data represents mean growth rates of biological triplicates $\pm \mathrm{SE}$ 
Figure 60: Pigment content ( $\mu \mathrm{g} / \mathrm{mg}$ dry weight) of $C$. fritschii cultures during A) UV$A$ acclimation and shock and B) UV-A shock only. Data points are mean values of biological triplicates \pm SE.. 128

Figure 61: MAA content ( $\mathrm{pg} / \mathrm{mg}$ dry weight) of $C$. fritschii cultures during A) UV-A acclimation and shock and B) UV-A shock only. Data points are mean values \pm SE.

Figure 62: Total number of detected peaks that show significant differences $(p<0.05)$ during acclimation and shock (total peaks $=293)$ and shock only (total peaks $=332)$ comparing 0 vs. $24 \mathrm{~h}$ (low UV-A or no UV-A) and 24 vs. $48 \mathrm{~h}$ (high UV-A) using a two-sample t-Test with equal variance.

Figure 63: Common metabolites identified in both UV-A acclimation \& shock and shock only cultures. Data points are mean values of biological replicates \pm SE. . ...133

Figure 64: Microscope images ( $\mathrm{x} 4$ lens) after $24 \mathrm{~h}$ and $48 \mathrm{~h}$ of growth of different cell densities of $\mathrm{HaCaT}$ cells in a 24 well plate.

Figure 65: Evaluation of IL-6 and IL-8 secretion in A \& B) TNF- $\alpha$ and C \& D) IL$1 \beta$ stimulated HaCaT cells. HaCaT's were exposed to concentrations of TNF- $\alpha(1,5$ and $\left.10 \mathrm{ng} \mathrm{mL}^{-1}\right)$ and IL-1 $\beta\left(0.1,0.5\right.$ and $\left.1 \mathrm{ng} \mathrm{mL}^{-1}\right)$ and vehicle control $\left(\mathrm{H}_{2} \mathrm{O}, 0 \mathrm{ng} \mathrm{mL}\right.$ $\left.{ }^{1}\right)$ for $24 \mathrm{~h}$. Cells were cultured in A \& C) 10\% FBS in DMEM and B \& D) $1 \%$ FBS in DMEM. Data represents mean values $(n=3) \pm$ standard deviation (SD).....146

Figure 66: Evaluation of A) IL-6 and B) IL-8 secretion in TNF- $\alpha$ stimulated HaCaT cells. HaCaT's were exposed to varying concentrations of TNF- $\alpha\left(0.01-10 \mathrm{ng} \mathrm{mL}^{-1}\right)$ and vehicle control $\left(\mathrm{H}_{2} \mathrm{O}\right)$ for $24 \mathrm{~h}$. Data represents mean values $(\mathrm{n}=3) \pm \mathrm{SD}$.

Figure 67: Hydrocortisone (HC) dose response of A) IL-6 and B) IL-8 secretion in TNF- $\alpha$ stimulated HaCaT cells. HaCaT's were exposed to varying concentrations of hydrocortisone $(10-0.0001 \mu \mathrm{M})$ or vehicle control (DMSO, $0 \mu \mathrm{M})$ for $24 \mathrm{~h}$ followed by incubation with TNF- $\alpha\left(10 \mathrm{ng} \mathrm{mL}^{-1}\right)$ and $\mathrm{HC}$ or vehicle control for a further $24 \mathrm{~h}$. Data represents mean values $(n=3) \pm S D$. 148

Figure 68: Anti-inflammatory method development workflow schematic. 149

Figure 69: Dose response of A) control $C$. fritschii pooled extracts and B) UV-B exposed $C$. fritschii extracts on IL-8 secretion in TNF- $\alpha$ stimulated HaCaT cells. HaCaT's were exposed to extracts or vehicle control (DMSO) for $24 \mathrm{~h}$ followed by incubation with TNF- $\alpha\left(1 \mathrm{ng} \mathrm{mL}{ }^{-1}\right)$ and extracts for a further $24 \mathrm{~h}$. Data represents mean values $(n=3) \pm S D$. 150

Figure 70: Effects of individual control C. fritschii extracts A) Hex, B) EtOAc, C) EtOH and D) $\mathrm{MeOH}$ on IL-8 secretion in TNF- $\alpha$ stimulated HaCaT cells. HaCaT's were exposed to extracts or vehicle control (DMSO) for $24 \mathrm{~h}$ followed by incubation with TNF- $\alpha\left(1 \mathrm{ng} \mathrm{mL}^{-1}\right)$ and extracts for a further $24 \mathrm{~h}$. Data represents mean values $(\mathrm{n}=3) \pm$ SD. 151

Figure 71: Effects of individual UV-B exposed $C$. fritschii extracts A) Hex, B) EtOAc, C) $\mathrm{EtOH}$ and D) $\mathrm{MeOH}$ on IL-8 secretion in TNF- $\alpha$ stimulated HaCaT cells. HaCaT's were exposed to extracts or vehicle control (DMSO) for $24 \mathrm{~h}$ followed by incubation with TNF- $\alpha\left(1 \mathrm{ng} \mathrm{mL}^{-1}\right)$ and extracts for a further $24 \mathrm{~h}$. Data represents mean values $(\mathrm{n}=3) \pm \mathrm{SD}$. 152 
Figure 72: Effect of m-gly on IL-8 secretion in TNF- $\alpha$ stimulated HaCaT cells. HaCaT's were exposed to m-gly $(3.124-50 \mu \mathrm{M}), \mathrm{HC}(10 \mu \mathrm{M})$ or vehicle control $\left(\mathrm{H}_{2} \mathrm{O}\right)$ for $24 \mathrm{~h}$ followed by incubation with TNF- $\alpha(1 \mathrm{ng} \mathrm{mL}-1)$ and m-gly for a further $24 \mathrm{~h}$. Data represents mean values $(n=3) \pm S D$

Figure 73: iCELLigence data of cell density experiment over approx. $48 \mathrm{~h}$. Data points are mean values of 2 replicates $(n=2)$.

Figure 74: Rate of HaCaT cell proliferation with A) control extract treatment and B) UV-B extract treatment. Gradient of the slope between 24 and $72 \mathrm{~h}$ was investigated. Data represents mean values of 2 replicates $(n=2)$.

Figure 75: Antibacterial activity of pooled $C$. fritschii control and UV-B extracts at concentrations of $0.5,0.25,0.125,0.063,0.031$ and $0.016 \mathrm{mg} \mathrm{mL}^{-1}$ vs. S. epidermis. DMSO was used as a vehicle control. Data represents OD values from one reading $(\mathrm{n}=1)$. 156

Figure 76: Antibacterial activity of $C$. fritschii control extracts: A) Hex, B) EtOAc, C) $\mathrm{EtOH}$ and D) $\mathrm{MeOH}$ at concentrations of $0.03125,0.0625,0.125,0.25$ and $0.5 \mathrm{mg}$ $\mathrm{mL}^{-1}$ with DMSO as a vehicle control against $S$. epidermis using the microdilution method. Values are mean \pm range of two replicates $(n=2)$ 157

Figure 77: Antibacterial activity of $C$. fritschii UV-B extracts: A) Hex, B) EtOAc, C) EtOH and D) $\mathrm{MeOH}$ at concentrations of $0.03125,0.0625,0.125,0.25$ and $0.5 \mathrm{mg} \mathrm{mL}^{-}$ ${ }^{1}$ with DMSO as a vehicle control against $S$. epidermis using the microdilution method. Values are mean \pm range of two replicates $(n=2)$. 158

Figure 78: Antibacterial activity of A) control (EtOH and $\mathrm{MeOH})$ and $\mathrm{B}) \mathrm{UV}-\mathrm{B}$ (EtOAc, EtOH and $\mathrm{MeOH}$ ) C. fritschii extracts at concentrations of 0.0625, 0.125 and $0.25 \mathrm{mg} \mathrm{mL}^{-1}$ against $S$. epidermis with DMSO as a vehicle control. Data represents mean \pm range of two replicates $(n=2)$. 159

Figure 79: Number of identified peaks extracted from $C$. fritschii biomass by chemical class using hexane, EtOAc, EtOH and $\mathrm{MeOH}$. 160

Figure 80: Visualisation of the biologically relevant metabolites identified in each solvent extracts using A) PCA, 2D scores plot showing 95\% confidence regions and B) Hierarchial heatmap visualisation; data is of the mean values of each extraction solvent; $\mathrm{MeOH}, \mathrm{Hex}, \mathrm{EtOAc}$ and $\mathrm{EtOH}$ from left to right. 161 


\section{List of Equations}

Equation 1: Specific growth rate $(\mu)$, where $N_{1}$ and $N_{2}$ are the dry biomass values at time $1\left(\mathrm{t}_{1}\right)$ and $2\left(\mathrm{t}_{2}\right)$ respectively.

Equation 2: Equations for the (a) calculation of resolution between two peaks and (b) calculation of the relative standard deviation $(\%)$.

Equation 3: Estimation of MAA concentration using the Beer-lambert law where; $\mathbf{A}$ $=$ absorbance at $\lambda \max$ (shinorine, $\lambda \max =334 \mathrm{~nm} ; \mathrm{m}$-gly, $\lambda \max =310 \mathrm{~nm}$ ), $\boldsymbol{\varepsilon}=$ extinction coefficient (shinorine, $\boldsymbol{\varepsilon}=44700 \mathrm{M}^{-1} \mathrm{~cm}^{-1} ; \mathrm{m}-\mathrm{gly}, \boldsymbol{\varepsilon}=28790 \mathrm{M}^{-1} \mathrm{~cm}^{-1}$ ), $\mathbf{c}=$ concentration, $\mathbf{l}=$ path length and $\mathbf{M W}=$ molecular weight (shinorine, $\mathbf{M W}=332.306$

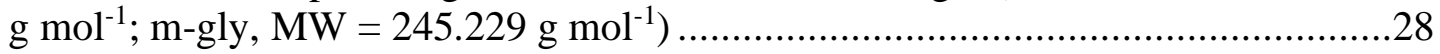

Equation 4: Equation for a) chl $a$ and b) total carotenoids concentrations. $\mathrm{A}_{(\mathrm{XXX})}=$ Absorbance at $665 \mathrm{~nm}, 470 \mathrm{~nm}$ or $666 \mathrm{~nm} . \mathrm{v}=$ volume of $\mathrm{MeOH}, 1=$ path length $(1$ $\mathrm{cm})$ and $\mathrm{V}=$ volume of sample.

Equation 5: Equations for the calculation of cell numbers (a) per $\mathrm{mL}$ of culture and (b) total per flask

Equation 6: Equations for the dilution of cells to experimental seeding density. (a) Calculation of dilution factor, (b) volume of initial cell volume needed and (c) volume of addition media needed.

Equation 7: General formula to calculate coefficient of variation (\%CV) to assess repeatability of assay. Where $\sigma=$ standard deviation and $\mu=$ mean. For ELISA assays a $\% \mathrm{CV} \leq 5$ and for protein assay a $\% \mathrm{CV} \leq 10$ shows good repeatability .46 


\section{Abbreviations}

$\mu \quad$ Specific growth rate

ALS Autosampler injector

AMDIS

Automated Mass Spectral Deconvolution and Identification System

ANOVA Analysis of variance

$\mathrm{aq}$

Aqueous

$\operatorname{ATCC}^{\circledR}$

American Type Culture Collection

BCA

Bicinchoninic acid

BSA

Bovine Serum Albumin

C. fritschii

Chlorogloeopsis fritschii

$\mathrm{CHCl}_{3}$

Chloroform

$\mathrm{Chl}$

Chlorophyll

$\mathrm{CO}_{2}$

Carbon dioxide

$\mathrm{CV}$

Coefficient of variation

d

Day

$\mathrm{Da}$

Daltons

DAD

Diode Array Detector

DEI

Desorption Electrospray Ionisation

DI

Direct Infusion

DMEM

Dulbecco's Minimum Eagle Media

DMSO

Dimethylsulfoxide

DNA

Deoxyribonucleic acid

dPBS

Dulbecco's Phosphate Buffered Saline

E. coli

Escherichia coli

EI

Electron ionisation

ELISA

Enzyme-Linked Immunosorbent Assays

ESI

Electrospray ionisation

EtOAc

Ethyl Acetate

$\mathrm{EtOH}$

Ethanol

FBS

Fetal Bovine Serum

GC-MS Gas chromatography-mass spectrometry

gly

Glycine

GMD

Golm Metabolome Database

GRAS

Genrally regarded as safe

$\mathrm{h}$

Hour

$\mathrm{H}_{2} \mathrm{O}$

Water

$\mathrm{H}_{2} \mathrm{O}_{2}$

Hydrogen peroxide

$\mathrm{HC}$

Hydrocortisone 


\begin{tabular}{|c|c|}
\hline $\mathrm{HCl}$ & Hydrochloric acid \\
\hline Hex & Hexane \\
\hline HPLC & High Performance Liquid Chromatography \\
\hline HRP & Horseradish Peroxidase \\
\hline IL & Interleukin \\
\hline IPTG & Isopropyl- $\beta$-D thiogalactopyranoside \\
\hline IR & Infrared \\
\hline KEGG & Kyoto Encyclopaedia of Genes and Genomes \\
\hline LC-MS & Liquid chromatography-mass spectrometry \\
\hline$m / z$ & Mass to charge \\
\hline MAA & Mycosporine-like amino acid \\
\hline MALDI & Matrix assisted laser desorption ionisation \\
\hline $\mathrm{MeCN}$ & Acetonitrile \\
\hline $\mathrm{MeOH}$ & Methanol \\
\hline m-gly & Mycosporine-glycine \\
\hline $\min$ & Minute \\
\hline MS & Mass spectrometry \\
\hline MSTFA & N-Methyl-N-trimethylsilyltrifluoroacetamide \\
\hline NIST & National Institute of Standards and Technology \\
\hline NMR & Nuclear magnetic resonance \\
\hline OD & Optical Density \\
\hline PAR & Photosynthetically Active Radiation \\
\hline PCA & Principle Component Analysis \\
\hline PCC & Pasteur Culture Collection \\
\hline ROS & Reactive oxygen species \\
\hline RSD & Relative standard deviation \\
\hline RTCA & Real-Time Cell Analyser \\
\hline s & Second \\
\hline S. epidermis & Staphylococcus epidermis \\
\hline SPE & Solid phase extraction \\
\hline TCA & Trichloroacetic acid \\
\hline TFA & Trifluoroacetic acid \\
\hline TMS & Trimethylsilyl \\
\hline TMCS & Trimethylchlorosilane \\
\hline TNF- $\alpha$ & Tumour necrosis factor $-\alpha$ \\
\hline TSB & Tryptic Soy Broth \\
\hline UV & Ultraviolet \\
\hline$v / v$ & Volume per volume \\
\hline$\varepsilon$ & Extinction coefficient \\
\hline$\lambda \max$ & Maximum aborbance \\
\hline$\sigma$ & Standard deviation \\
\hline
\end{tabular}




\section{Introduction}

\section{Cyanobacteria}

Cyanobacteria, also known as blue-green algae, are gram-negative bacteria that have the ability to photosynthesise [1]. They have evolved over billions of years and are thought to be the oldest microorganisms creating the earth's oxygenic atmosphere and responsible for the evolution of eukaryotic photosynthesis $[1,2]$. These photosynthetic organisms' are primary producers that contribute largely to global productivity $[3,4]$. With diverse morphologies, cyanobacteria encompass a variety of forms including rod, spherical and spirals as unicellular; Microcystis sp., Synechococcus sp. and Synechocystis sp. or filamentous species; Anabaena sp., Nostoc sp. and Athrospira platensis [5]. Taxonomically they are distributed into the five major sub-sections: Subsection I (order: Chroococcales), II (order: Pleurocapsales), III (order: Oscillatoriales), IV (order: Nostocales) and V (order: Stigonematales) [5,6]. With much debate on the classification of cyanobacteria, newer systems using phylogenetic analyses are constantly evolving $[7,8]$.

Cyanobacteria have adapted to survive in an array of ecological habitats such as marine, terrestrial and freshwater environments which can involve exposure to extreme environmental surroundings [9]. They have adapted to survive and thrive under biotic and abiotic stress conditions such as ultraviolet (UV) radiation, high and low light and varying temperatures [6].

Extremophilic cyanobacteria can be defined as thermophiles (high temperature tolerant), psychrophiles (cold tolerant), acidophiles (low $\mathrm{pH}$ tolerant), alkaliphiles (high $\mathrm{pH}$ tolerant) and halophiles (salt tolerant) [9]. Examples include the species Calothrix sp. and Leptolyngbya foveolaru living in high mountain habitats, dealing with temperature differences, limited water retention and high UV radiation [10]. In cold environments such as polar regions species such as Chroococcidiopsis sp. are adapted to persistent low temperatures, limited light, and high UV radiation $[9,11]$.

Adaptation of cyanobacteria to varying conditions is due to many factors including alterations in metabolism and production of unique secondary metabolites. 


\section{Cyanobacterial metabolism}

Cyanobacteria are photoautotrophic and perform oxygenic photosynthesis as their main source of energy in light conditions utilising water $\left(\mathrm{H}_{2} \mathrm{O}\right)$, carbon dioxide $\left(\mathrm{CO}_{2}\right)$, inorganic compounds and light. They also have the ability to switch to different means of metabolism in different conditions. Heterotrophic growth in dark conditions produces energy by use of organic carbon substrates in fermentation reactions [1]. The specialised cells, heterocysts, allow some filamentous species to perform nitrogen fixation by conversion of atmospheric nitrogen into ammonia [12].

Primary metabolites are involved in primary metabolism which is essential for growth within all organisms. End products of primary metabolism result in energy and intermediate production for the synthesis of proteins, lipids and carbohydrates [13].

Cyanobacteria are a prolific source of secondary metabolites. Also known as natural products these are not required directly for an organisms' primary metabolism but aid in survival by providing an advantage over other species [14]. They are often unique and species specific.

There is some overlap between the two terms (primary and secondary) where some metabolites are essential for primary metabolism that are specific and present during abiotic and biotic pressures [6].

Cyanobacteria have many adaptive capabilities to survive during environmental stress. Most interestingly is the rearrangement of metabolism and production of secondary metabolites. These unique metabolites are structurally diverse including; alkaloids, terpenoids, polyketides, peptides and UV-absorbing compounds. They possess a wide variety of functions to protect cyanobacterial cells such as photoprotection, defence against grazers, chemosensory and may also act as antioxidants[6,15]. There is much interest related to the use of cyanobacterial metabolites in industrial biotechnology as sustainable sources of natural ingredients [16] which will be discussed below and in

\section{Chapter 5.}




\begin{abstract}
Abiotic stress
Abiotic stress is the change in environmental parameters that affect cellular homeostasis in cyanobacteria and other organisms [17]. Adaptation and survival in these conditions are a result of many mechanism including metabolic pathway shifts [17]. Many studies have been carried out to determine the effects of abiotic stress on cyanobacterial growth and metabolism including salt stress on trehalse production in Nostoc commune [18], heavy metal stress on Anabaena flos-aquae [19] and UV and osmotic stress on Chlorogloeopsis fritschii, (C. fritschii), PCC 6912 [20]. There is much interest in the use of abiotic stressors such as UV in cyanobacterial research to induce protective mechanisms to enhance the production of secondary metabolites which have potential for industrial purposes [21,22].
\end{abstract}

\title{
Ultraviolet radiation
}

UV radiation is the most common abiotic stress applied to cyanobacteria. This is due to cyanobacteria's presence on earth during the Precambrian era, at a time with an absence of the ozone layer, which led to the adaptation to high solar UV radiation [23]. Solar light that reaches the earth's surface (terrestrial and marine/freshwater environments) contains photosynthetically active radiation (PAR, 400-700 nm), infrared radiation (IR, $>700 \mathrm{~nm}$ ) and approx. 5\% UV. 95\% of which is comprised of UV-A (320-400 nm) and 5\% of UV-B (280-320 nm) [24]. The majority of UV-B is absorbed by the stratospheric ozone layer (Figure 1).

The effect of UV on cyanobacteria has been widely researched including the interaction with biomolecules, production of reactive oxygen species (ROS) which cause oxidative stress, impaired growth, partial inhibition of photosynthesis and decreased enzyme activity [24,25].

Although a smaller proportion of UV-B reaches the earth, it has a more damaging effect on all organisms. A shorter wavelength results in higher energy $(E=\lambda v)$ owing to UV-B's direct and indirect damage (Figure 1) whereas UV-A causes indirect damage by the production of ROS. 
Cyanobacteria have many protective strategies to counteract the effects of UV radiation including avoidance, programmed cell death, DNA repair and production of the photoprotective compounds, such as mycosporine-like amino acids (MAAs) $[26,27]$. They also produce antioxidants which counteract the oxidative damage caused by ROS [2]. UV radiation therefore also has a role as an activator of secondary metabolite production such as MAAs [23] and other protective secondary metabolites [28].

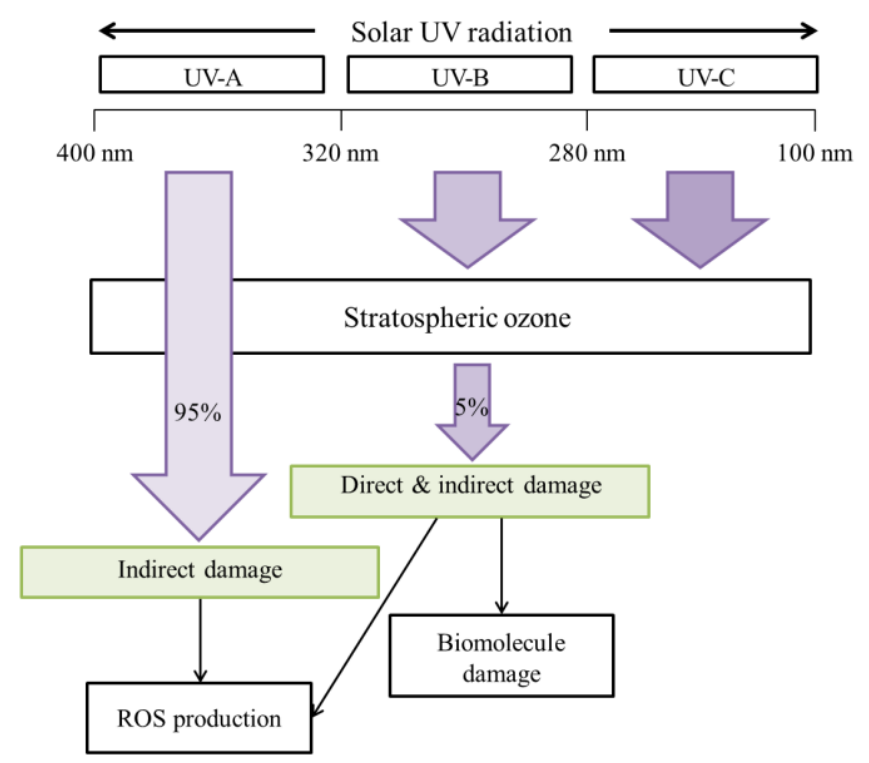

Figure 1: Effect of Ultraviolet (UV) radiation on cyanobacteria

\section{Mycosporine-like amino acids (MAAs)}

MAAs are water soluble, low molecular weight $(<400 \mathrm{Da})$ colourless molecules produced by cyanobacteria and other organisms including microalgae, macroalgae and fungi [29]. They have strong absorption in the UV region (310-365 nm) with high molar extinction coefficients $\left(\varepsilon=28100-500001 \mathrm{~mol}^{-1} \mathrm{~cm}^{-1}\right)$ (Table 1) [30]. They have the ability to absorb harmful UV and dissipate the energy without the production of ROS [23].

They are a group of around 20 molecules which consist of a cyclohexenone or cyclohexenimine central ring that is responsible for absorption of the high energy UV photons [31]. Conjugation to nitrogen substituents from amino acids or imino alcohols also allows varying absorptions [29,31] (Table 1). 
Table 1: Examples of MAAs produced by cyanobacteria [6,29,31]

\begin{tabular}{llll}
\hline MAA & $\begin{array}{l}\lambda \max (\mathrm{nm}), \\
\varepsilon\left(1 \mathrm{~mol}^{-1} \mathrm{~cm}^{-1}\right)\end{array}$ & $\begin{array}{l}\text { Species of } \\
\text { cyanobacteria }\end{array}$ \\
\hline Mycosporine- & 310,28800 & $\begin{array}{l}\text { C. fritschii, PCC } \\
\text { glycine }\end{array}$ \\
Porphyra-334 [20] & \\
Asterina-330 & & C. fritschii, PCC \\
\end{tabular}

There are two biosynthetic routes involved in the production of MAAs (Figure 2). The first is the shikimate pathway (biosynthesis of aromatic acids) [33]. Deoxy-Darabinoheptulosonate-7-phosphate (DAHP) is formed from phosphoenolpyruvate (PEP) and erythrose-4-phosphate (E4P) using DAHP synthase. DAHP is then converted to 3-dehydroquinate and subsequent transformation into 4-deoxygadusol (4DG). The primary MAA mycosporine-glycine (m-gly) is then formed from the addition of glycine to 4-DG which can be further converted into a secondary MAA by addition of other amino acids such as serine (to produce shinorine) and threonine (to produce porphyra-334) [34]. The other pathway involves the pentose-phosphate 
pathway by forming the common intermediate 4-DG from sedoheptulose-7-phosphate via 2-epi-5-epi-violiolone [28].

1) Shikimate pathway
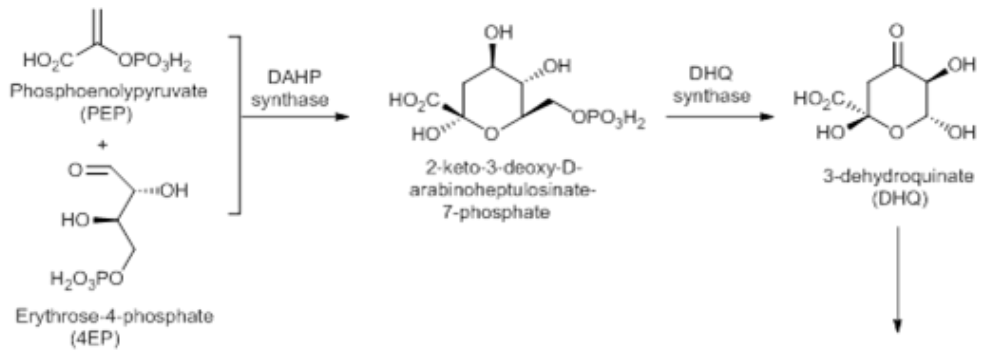

2) Pentose phosphate pathway
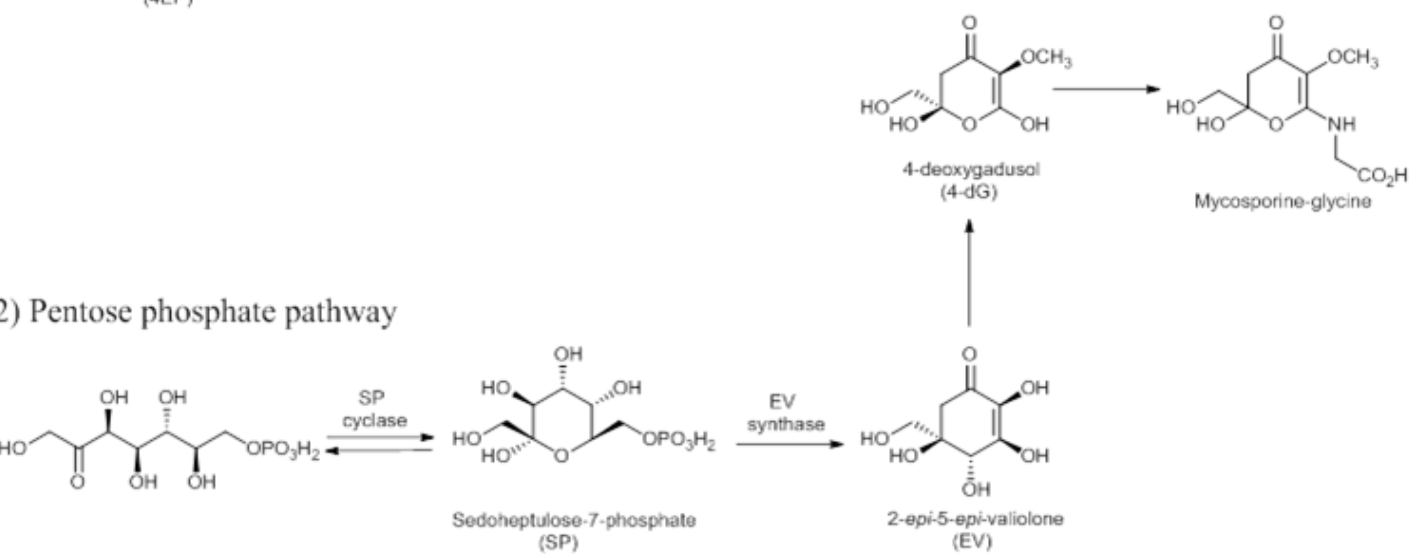

Figure 2: Biosynthetic pathways for the production of mycosporine-like amino acids (MAAs) via 1) Shikimate pathway and 2) pentose phosphate pathway via the common intermediate 4-deoxygadusol (4-DG) [28].

To understand secondary metabolite production, primary metabolite levels need to be investigated which will be discussed further throughout the thesis within Chapters 2, 3 and 4. 


\section{Metabolomics}

Metabolomics is the newest member of the -omics group which includes genomics, proteomics and transcriptomics. It is a systems biology technology for the study of metabolism in a variety of biological systems. It is used to estimate and analyse the low molecular weight metabolites $(<1000 \mathrm{Da})$ produced by biological samples such as; whole organisms, cells, tissues and biological fluids [35,36]. As it closely relates to the physiology of an organism, it can be used to analyse the altered metabolite levels in respect to external stimuli. This principle has been thoroughly developed within human health for biomarker discovery for improved diagnostics of diseases including cancer [37] and cardiovascular diseases [38]. It is also used in chemical ecology [39], plant sciences [40] and in microbial research [41].

There are five main types of analysis used in metabolomics research [36]:

i. Targeted analysis: Pre-selection of specific metabolites which are usually involved in common pathways.

ii. Metabolite profiling: Investigates a group of known metabolites using a common technique.

iii. Metabolite fingerprint: A non-biased, untargeted approach to analyse intracellular metabolite pools, often referred to as endo-metabolome.

iv. Metabolite footprint: Analysis of excreted metabolites (extracellular metabolites), usually referred to as exo-metabolome analysis.

v. Flux analysis: Application of stable isotopes $\left({ }^{13} \mathrm{C},{ }^{15} \mathrm{~N}\right.$ or $\left.{ }^{18} \mathrm{O}\right)$ to analyse pathways in organisms or cells.

A detailed experimental design is essential for successful metabolomics investigations. From biological material to sampling and quenching of metabolism, sample preparation, sample analysis using high throughput analytical techniques, data export and data analysis [42]. 


\section{Cell harvest, quenching and metabolite extraction}

In order to capture a true in situ metabolome of an organism, a snap-shot of the cellular metabolic activity at an exact time point must be taken. This is a difficult aspect of the analysis as the metabolites are constantly changing over time with changes in environmental conditions. Steps for harvesting and inactivation need to be rapid. Light intensity, temperature and other environmental conditions must be maintained in order to prevent metabolite degradation [43].

Many different methods of harvesting (removal of cells from growth media) followed by quenching (halting metabolism) can be achieved [44]. These include; fast filtration and centrifugation which can be done at cold temperatures to separate and remove cells from growth media [45] or using a super cooled $\left(-50^{\circ} \mathrm{C}\right)$ buffer such as $70 \%$ cold methanol $(\mathrm{MeOH})$ [44] to quench before separation of cells from media [45]. These cells and supernatant are then preserved using liquid nitrogen or freezing at $-80{ }^{\circ} \mathrm{C}$ followed by sample concentration by freeze drying [44]. The efficiency of these protocols are dependent on the microorganism [46].

Extraction of metabolites from cyanobacterial cells and other microorganisms is dependent on the analysis to be used. No single method has the ability for efficient global extraction of all metabolites [47]. A variety of cell disruption techniques combined with solvent extractions have been evaluated for untargeted and targeted metabolomics (Table 2).

Method development for the extraction of metabolites from C. fritschii for gaschromatography-mass spectrometry (GC-MS) analysis will be discussed further at the beginning of Chapter 2. 
Table 2: Untargeted and targeted metabolite extraction methods in varying cyanobacterial species.

\begin{tabular}{|c|c|c|c|c|}
\hline Metabolite & $\begin{array}{c}\text { Species of } \\
\text { cyanobacteria }\end{array}$ & Extraction & Technique & Reference \\
\hline \multirow[t]{4}{*}{ MAAs } & $\begin{array}{l}\text { Chlorogloeopsis } \\
\text { fritschii }\end{array}$ & $\begin{array}{l}25 \%(\mathrm{v} / \mathrm{v}) \mathrm{MeOH}_{(\mathrm{aq})} \\
\text { with shaking at } 45^{\circ} \mathrm{C}\end{array}$ & HPLC & [48] \\
\hline & Lyngbya sp., & $\begin{array}{c}100 \% \mathrm{MeOH}, \\
\text { incubation at } 4{ }^{\circ} \mathrm{C} \text {. }\end{array}$ & HPLC & [49] \\
\hline & $\begin{array}{l}\text { Leptolyngbya } \\
\text { sp. }\end{array}$ & $\begin{array}{c}100 \% \mathrm{MeOH}, \\
\text { incubation at } 4{ }^{\circ} \mathrm{C} \text {. }\end{array}$ & LC-MS & {$[50]$} \\
\hline & Nostoc sp. & $\begin{array}{c}100 \% \mathrm{MeOH} \\
\text { incubation at } 4{ }^{\circ} \mathrm{C} \\
\text { followed by sonication }\end{array}$ & $\begin{array}{l}\text { HPLC, } \\
\text { LC-MS }\end{array}$ & {$[32]$} \\
\hline \multirow[t]{2}{*}{ Pigments } & $\begin{array}{l}\text { Chlorogloeopsis } \\
\text { fritschii }\end{array}$ & $\begin{array}{l}100 \% \text { acetone with } \\
\text { bead beating }\end{array}$ & $\begin{array}{l}\text { HPLC, } \\
\text { LC-MS }\end{array}$ & [51] \\
\hline & $\begin{array}{c}\text { Nostoc } \\
\text { punctiforme }\end{array}$ & $\begin{array}{c}100 \% \text { acetone }\left(-20^{\circ} \mathrm{C}\right) \\
\text { with sonication }\end{array}$ & HPLC & {$[52]$} \\
\hline \multirow[t]{3}{*}{$\begin{array}{l}\text { Intracellular } \\
\text { metabolites }\end{array}$} & $\begin{array}{c}\text { Nostoc } \\
\text { punctiforme }\end{array}$ & $\begin{array}{l}\mathrm{MeOH}: \mathrm{CHCl}_{3}: \mathrm{H}_{2} \mathrm{O} \\
\text { with sonication, bead } \\
\text { beating and shaking }\end{array}$ & GC-MS & [52] \\
\hline & $\begin{array}{l}\text { Synechocystic } \\
\text { sp. }\end{array}$ & $\begin{array}{c}\mathrm{MeOH}: \mathrm{CHCl}_{3}: \mathrm{H}_{2} \mathrm{O} \\
\text { with agitation at } 37^{\circ} \mathrm{C} \text {. }\end{array}$ & GC-MS & {$[53]$} \\
\hline & $\begin{array}{c}\text { Synechococcus } \\
\text { elongatus }\end{array}$ & $\begin{array}{c}\mathrm{MeCN}: \mathrm{MeOH}: \mathrm{H}_{2} \mathrm{O} \\
\text { (40:40:200) + 0.1 M } \\
\text { formic acid with } \\
\text { sonication }\end{array}$ & LC-MS & {$[54]$} \\
\hline \multirow[t]{2}{*}{$\begin{array}{l}\text { Extracellular } \\
\text { metabolites }\end{array}$} & $\begin{array}{c}\text { Nostoc } \\
\text { punctiforme }\end{array}$ & $\begin{array}{c}\text { 9:1:0.001 } \\
\left(\mathrm{H}_{2} \mathrm{O}: \mathrm{MeCN}: \text { formic }\right. \\
\text { acid; v/v/v) }\end{array}$ & LC-MS & {$[55]$} \\
\hline & $\begin{array}{c}\text { Synechococcus } \\
\text { elongatus }\end{array}$ & $\begin{array}{l}\text { Solid phase extraction } \\
\text { (SPE) }\end{array}$ & LC-MS & {$[54]$} \\
\hline
\end{tabular}




\section{Analytical techniques}

The two most successful techniques in metabolomics include nuclear magnetic resonance (NMR) and mass spectrometry (MS). NMR has lower sensitivity than MS (described below) but can detect and quantify more abundant compounds within samples [56]. The sample itself can be re-used which is important in experiments with limited amounts. Many compounds are hard to ionise and derivatisation is needed in MS which can be avoided with NMR. Structure elucidation of unknowns is also possible, including stereochemistry [56,57].

MS based metabolomics can analyse a variety of biological samples at speed, with high sensitivity and a wide dynamic range [58]. MS generates gas phase ions by use of ionisation techniques such as; electrospray ionisation (ESI-MS) and electron ionisation (EI-MS). Ions are accelerated into a magnetic field and are separated according to their mass-to-charge ratio $(\mathrm{m} / \mathrm{z})$. Ions are then detected at different intensities. Separation free MS technologies include direct infusion (DI)-MS, matrix assisted laser desorption ionisation (MALDI) MS and desorption electrospray ionisation (DEI) MS [42].

MS is most commonly used in combination with high resolution separation techniques including liquid chromatography (LC) and gas chromatography (GC) [42].

GC-MS is the most standardised method in metabolomics [59] and the technique of choice within this research project. Analysis of low molecular weight metabolites (<650 Da) including; acids, alcohols, hydroxyl-acids, amino acids, sugars, fatty acids and sterols can be achieved [59]. It does not require prior knowledge (i.e. genomic data) and can be applied to a variety of biological or environmental samples especially with limited amounts. It is highly sensitive with high resolution and reproducibility with repeatable fragmentation patterns produced, which allows for metabolite identification [58].

Disadvantages include the need for sample processing and derivatisation of nonvolatile compounds. Derivatisation is required to increase thermal stability and volatility of polar metabolites. Trimethylsilation and methoxymation are the most common types of derivatisation methods [58].

Active hydrogens, $-\mathrm{COOH},-\mathrm{OH},-\mathrm{NH}$ and $-\mathrm{SH}$, can be protected by replacement with alkylsilyl groups to produce trimethylsilyl (TMS) derivatives. These TMS derivatives 
produce better mass spectral profiles. Methoxymation provides protection of exchangeable protons and converts carbonyl groups into oximes which can result in stabilisation of $\alpha$-ketoacids and open-ring conformation of sugars. Conversion reactions can also occur as well as formation of artefacts that can interfere with data interpretation $[42,60]$.

Advantages of GC-MS include the standardised public libraries that have been extensively developed to include mass spectral data and retention times of many different chemicals. The most popular includes the publicly available NIST Mass Spectral Library collection of the U.S. National Institute of Standards and Technology (NIST) [61]. Other examples include the Golm Metabolome Database (GMD) [62].

Open access, and commercial software/websites are readily available for peak alignment, extraction, identification and pathway analysis of GC-MS data (and other MS techniques). All software/websites will provide varying results which affect the interpretation [58] and therefore reporting of all metadata associated with metabolomics experiments is important to provide biological and empirical context of the data. Proposed minimum reporting standards for chemical analysis have been reported by the Chemical Analysis Working Group (CAWG) as part of the Metabolomics Standards Initiative (MSI) [63].

In this thesis a consistent freely available workflow was employed for all experimental data analysis (Figure 3). 

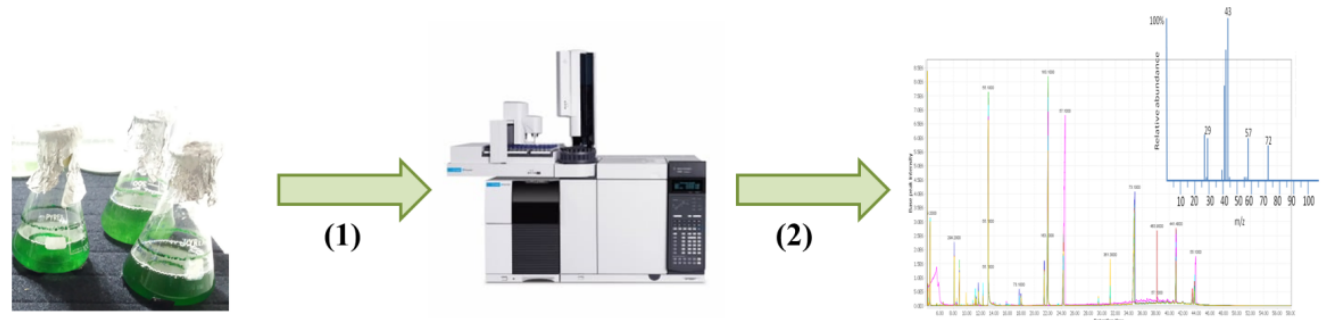

\section{Metabolite profiling workflow}

(2)
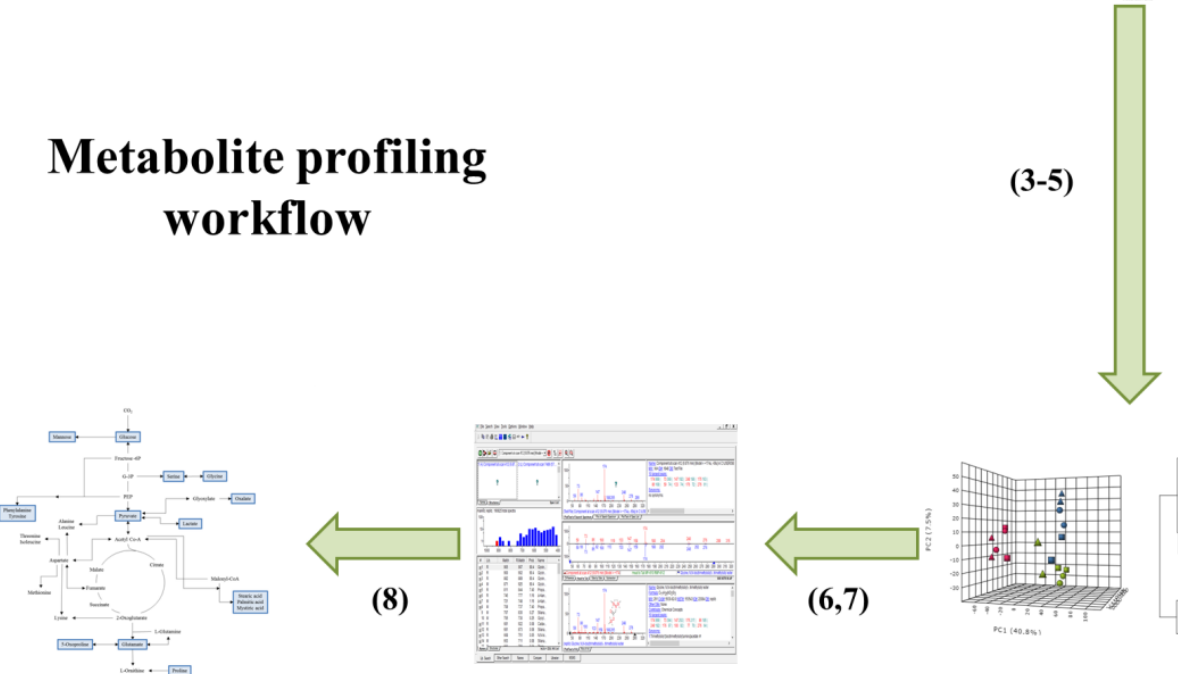

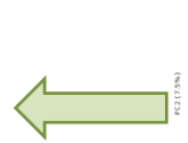

$(6,7)$

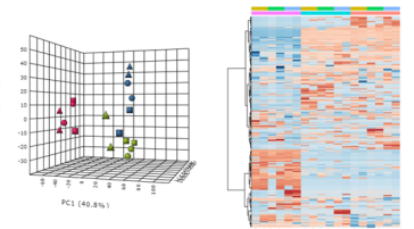

Figure 3: Final metabolite profiling workflow using GC-MS. After cultivation and harvest of biomass; (1) metabolite extraction and derivatisation, (2) GC-MS data acquisition, (3) deconvolution and peak detection using AMDIS, (4) conservative peak alignment using SpectConnect, (5) statistical analysis using MetaboAnalyst (PCA, ANOVA, heatmap) and Excel (t-Test), (6) semi-quantification by normalisation to dry weight (intracellular only) and internal standard, (7) identification using GMD and NIST library and (8) pathway analysis of biologically relevant metabolites (KEGG and MetaboAnalyst). 


\section{Cyanobacterial metabolomics}

As metabolomics closely relates to the physiology of an organism, it is useful in cyanobacterial research due to their adaptation to a variety of biotic and abiotic factors (as described above) [43].

Many studies have been conducted to identify targeted intracellular metabolites within cyanobacteria during abiotic stress. Investigations include the production of MAAs using high performance-liquid chromatography (HPLC) in Lyngbya sp. CU2555, Nostoc commune, Anabaena variabilis PCC 7937, Calothrix sp. and C. fritschii PCC 6912 [10,48,49,64,65], pigment concentrations using UV-visible spectroscopy in Nostoc flagelliforme [66] and pigment composition of Nostoc punctiforme ATCC 29133 using HPLC [52].

Less research has been conducted on metabolite profiling of intracellular metabolites within cyanobacteria. Some examples include; the analysis of the model cyanobacterium Synechocystis sp. PCC 6803 from high to low $\mathrm{CO}_{2}$ using GC-MS [53], GC-MS analysis of Nostoc punctiforme ATCC 29133 during UV-A exposure [52] and a comparison between strains using both LC-MS and GC-MS; Synechococcus elongates PCC 7942, Synechococcus sp. PCC7002 and Synechocystis sp. PCC6803 [67].

Cyanobacteria, like many other microorganisms release carbon-based primary and secondary metabolites into their surroundings. Released metabolites are by-products of metabolism. The uptake and release of metabolites change with varying environments [54]. Monitoring industrially relevant metabolites released by microorganisms into their surroundings is a widely used technique in the fermentation industry. It can be used in bioprocess monitoring, fermentation biomarker identification, for monitoring metabolite levels in fermentation processes and microbial contamination $[68,69]$.

Combining intracellular and extracellular analysis is useful to provide a more holistic picture of metabolite production during growth and in response to different environmental conditions [54,69,70] (Chapter 3). 


\section{Industrial biotechnology}

With the depletion of fossil fuels and growing concerns of global warming, a more sustainable approach is necessary to continue to produce consumer goods and pharmaceuticals for growing global markets. A considerable amount of research has been conducted to find new sources of industrially relevant compounds to reduce the carbon footprint and increase sustainability by replacing oil-based ingredients with naturally derived products $[21,22]$.

Cyanobacteria have received much interest in becoming a promising alternative due to their diversity, simple growth needs and simple genetic background which are easily manipulated to form cell factories [1,71]. Many industrial processes rely on heterotrophic bacteria to produce many different useful compounds. These fermentation processes rely on carbon substrates which can be costly. Cyanobacteria are able to utilise $\mathrm{CO}_{2}$ from flue gas as a carbon source for photosynthesis [1,72].

The main drawback with the use of cyanobacteria in industry is the difficulty in achieving regular high production yields for commercialisation. The use of bioengineering to increase yields has been researched by introducing enzymes from different organisms to increase production of valuable compounds $[1,72]$.

Useful compounds include bulk (commodity) chemicals of low value but high productivity to high value products that are usually produced in low concentrations (Figure 4). 


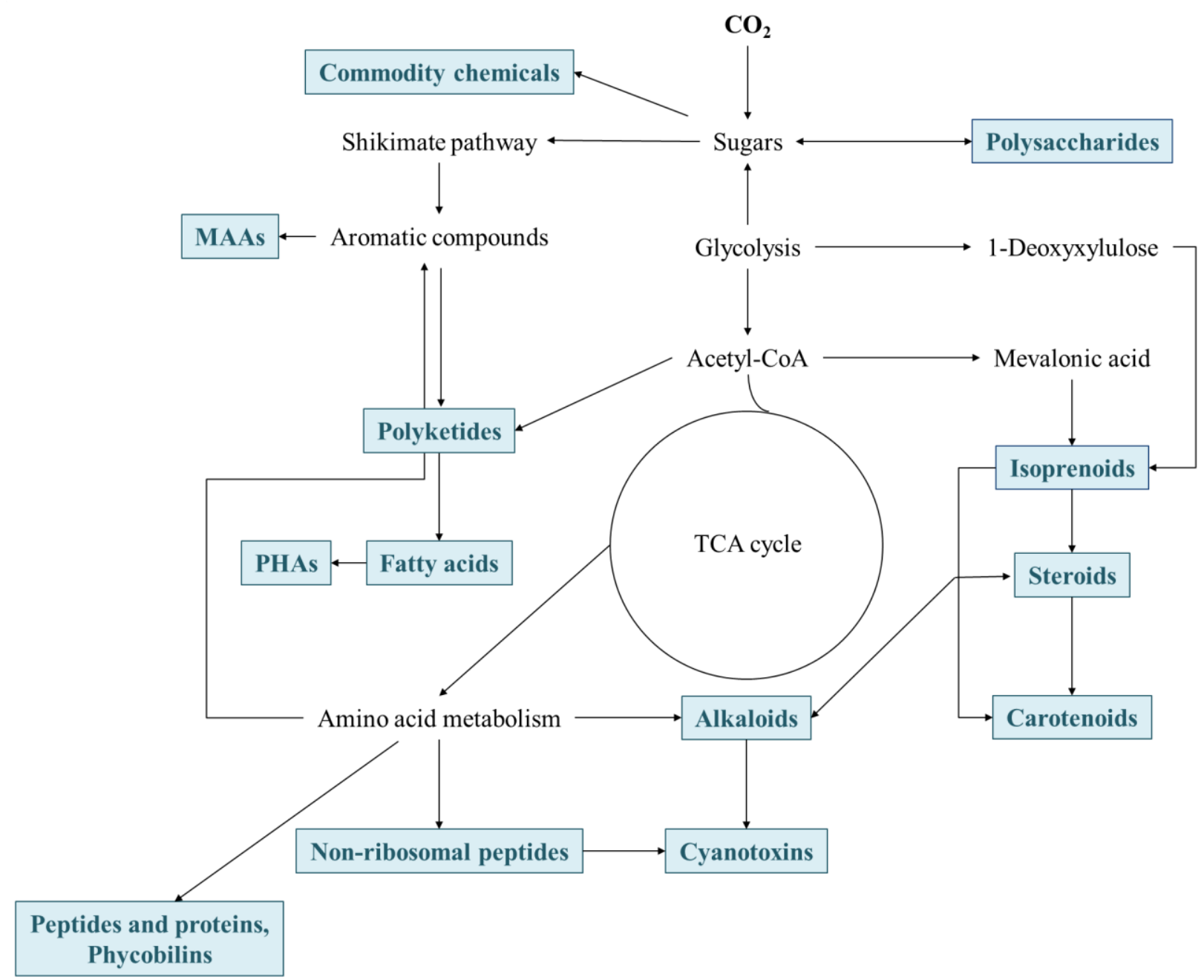

Figure 4: Generalised biosynthetic pathway schematic of key metabolite production from $\mathrm{CO}_{2}$ assimilation in cyanobacteria. 
Some strains of cyanobacteria are readily used in industry, including the edible Arthrospira (Spirulina) and Nostoc which have been used as a food source for thousands of years [73].

Spirulina has been well researched for its application within industry. With a GRAS status (Generally Regarded As Safe) it is used as a health food due to its extensive source of proteins, polyunsaturated fatty acids ( $\gamma$-linoleic acid, GLA), antioxidants (phycocyanin and carotenoids) and vitamins [74].

C. fritschii is a less well-known species and a potential candidate for use in industrial biotechnology. C. fritschii is a sub-section V cyanobacterium, first isolated from soil crusts in India with diverse morphology and function [75]. This strains potential is due to its robustness and scalability [76]. It is moderately thermophilic which enables growth at higher temperatures thus reducing contamination risk by other nonthermophilic species [20] and tolerant to a variety of growth conditions $[20,51,75]$. It also has auto-flocculating capabilities providing an easier and cheaper way of large scale separation [76]. A complete metabolome analysis has not yet been conducted on C. fritschii which would assist in determining its feasibility within the biotechnology industry.

Natural products from cyanobacteria usually have bioactive properties. They can be used in the pharmaceutical industry as lead compounds in drug discovery. They can be used as templates for synthesis of new drugs to treat complex diseases. Cyanobacteria have been widely researched for their applications in this field. They have found to possess a wide range of potential antimicrobial, anticancer, antiviral and anti-inflammatory activities [16] (Table 3). 
Table 3: Potential applications of cyanobacterial natural products in pharmaceuticals and beauty personal care products $[6,16]$.

\begin{tabular}{|c|c|c|c|}
\hline $\begin{array}{c}\text { Species of } \\
\text { cyanobacteria }\end{array}$ & $\begin{array}{l}\text { Bioactive } \\
\text { compound }\end{array}$ & Biological activity & References \\
\hline \multirow[t]{3}{*}{ Spirulina platensis } & Spirulan & Antiviral & [13] \\
\hline & $\gamma$-linolenic acid & $\begin{array}{c}\text { Precursor to } \\
\text { prostaglandins }\end{array}$ & [77] \\
\hline & Phycocyanin & Cosmetic colourants & [30] \\
\hline Lyngbya majuscule & Apratoxins & Anticancer & \\
\hline \multirow[t]{3}{*}{ Nostoc commune } & Nostodione & Antifungal & [13] \\
\hline & Carotenoids & Antioxidant & [74] \\
\hline & MAAs & Sunscreen & [78] \\
\hline Anabaena circinalis & Anatoxin-a & Anti-Inflammatory & {$[13,16]$} \\
\hline \multirow{3}{*}{$\begin{array}{c}\text { Fischerella } \\
\text { muscicola }\end{array}$} & Fischerellin & Antifungal & [16] \\
\hline & Scytonemin & Anti-inflammatory & [79] \\
\hline & & Anti-proliferation & \\
\hline
\end{tabular}

Secondary metabolites can also be used as natural ingredients in the cosmetics industry. Uses include the photoprotective MAAs in sunscreens to protect the skin from harmful UV exposure [31]. Pigments such as carotenoids and phycobiliproteins can be used as natural colourants but also as antioxidants to protect the skin from damage caused by UV exposure [16].

A challenge remains in assessing and understanding the ability of cyanobacteria to produce target metabolites in sufficient quantities under repeatable conditions.

By combining both metabolomics and bioactivity via in vitro assays, other cyanobacterial strains, including $C$. fritschii, can be profiled for their potential use in industrial biotechnology (Chapter 5). 


\section{Aims and objectives}

The overall aim of this thesis is to evaluate the metabolite profile of the less investigated cyanobacterium Chlorogloeopsis fritschii (C. fritschii) PCC 6912, as a potential candidate for use in industrial biotechnology. To construct a GC-MS workflow to evaluate the changes in low molecular weight metabolite levels of $C$. fritschii over varying time-series under standard and UV stress growth conditions. The main goal is to identify biologically relevant metabolites with roles in cyanobacterial metabolism for evaluation of growth phases, adaptation to UV stress and applications for use in consumer goods.

A brief description of each chapter, including specific ojectives, is presented below:

\section{Chapter 1: Materials and Methods}

This chapter includes all general materials and methods used throughout the research study. Additional information about design of experiments specific to each research topic is included at the beginning of each chapter.

Chapter 2: Time-Series Metabolite Level Investigation of Chlorogloeopsis fritschii, PCC 6912, During Standard Growth Conditions

The first results chapter is an investigation into changes in the low molecular weight metabolite levels of $C$. fritschii over varying time-series during standard growth conditions. Firstly, extraction of metabolites were optimised for GC-MS analysis this was followed by an assessment into the changes in metabolite levels over short term $(48 \mathrm{~h})$ and long term cultivation (120 d) as well as between axenic and xenic cultures of C. fritschii (28 d).

Chapter 3: Intracellular and Extracellular Metabolite Level Changes of Chlorogloeopsis fritschii, PCC 6912, During Ultraviolet (UV) A and UV-B Exposure

The main objective was to investigate the changes in intracellular and extracellular metabolite levels changes of $C$. fritschii during UV-B exposure and intracellular metabolite level changes during UV-A exposure over $48 \mathrm{~h}$. The $48 \mathrm{~h}$ standard growth analysis in Chapter $\mathbf{2}$ is also referenced as a control experiment. 
Chapter 4: Acclimation vs. Shock: The Stress Response of Chlorogloeopsis fritschii, PCC 6912, to Ultraviolet Radiation

This chapter investigates the response of $C$. fritschii to pre-acclimation to low dose UV followed by shock UV exposure. The main objective was to evaluate the metabolite level changes and MAA levels during a low dose UV acclimation phase followed by shock UV exposure to assess shock response and tolerance of $C$. fritschii to UV exposure.

Chapter 5: Bioactivity Assessment of Chlorogloeopsis fritschii, PCC 6912, for use in Consumer Goods

The final results chapter assesses extracts from $C$. fritschii in in-vitro assays to evaluate anti-inflammatory, proliferation and antibacterial activity with complementary GCMS analysis for further elucidation of active ingredients with relevance to personal care products.

\section{Chapter 6: General Discussion}

A final discussion on the results including the potential application of $C$. fritschii in industrial biotechnology. 


\section{Chapter 1: Materials and Methods}

\subsection{Organism and growth conditions}

The cyanobacterium Chlorogloeopsis fritschii (C. fritschii), PCC 6912, was obtained from Pasteur Culture Collection (PCC) and grown in autoclaved deionised water with filtered BG-11 [5] growth medium (Product number: C3061, Sigma Aldrich, UK). The strain was maintained as a master culture in $50 \mathrm{~mL} \mathrm{BG}-11$ at a temperature of $27^{\circ} \mathrm{C} \pm$ $2{ }^{\circ} \mathrm{C}$ under continuous PAR illuminated at $15 \mu \mathrm{mol} \mathrm{m} \mathrm{m}^{-2} \mathrm{~s}^{-1}$ (measured using a PAR light sensor, Enviromonitors, UK). Experimental cultures were pre-grown in $300 \mathrm{~mL}$ BG-11 media under the same conditions with constant shaking at $80 \mathrm{rpm}$. Growth was monitored using optical density at $750 \mathrm{~nm}\left(\mathrm{OD}_{750 \mathrm{~nm}}\right)$ using a UV-visible spectrophotometer (Shimadzu, UV-2550, Kyoto, Japan). pH was also measured throughout growth using $\mathrm{pH}$ indicator paper (Fisherbrand ${ }^{\mathrm{TM}}$, product no. 10642751).

\subsection{Sample harvest and growth analysis}

Forty $\mathrm{mL}$ volumes (unless otherwise stated) of experimental cultures were harvested in triplicate (biological replicates) at each time point by centrifugation at $4400 \mathrm{rpm}$ for $20 \mathrm{~min}$ to produce a pellet and supernatant which were stored at $-80{ }^{\circ} \mathrm{C}$. The supernatants $(40 \mathrm{~mL})$ were collected and freeze-dried (Edwards, super modulyo) for $72 \mathrm{~h}$. The remaining pellets were transferred into pre-weighed Eppendorf's and freeze dried for $24 \mathrm{~h}$ (Scanvac, CoolSafe ${ }^{\mathrm{TM}}$, LaboGene ${ }^{\mathrm{TM}}$, Vassingerød, Denmark) and reweighed for dry weight measurements. Both pellets and dried supernatant were stored at $-20{ }^{\circ} \mathrm{C}$ until analysis. Specific growth rate of the cultures during each experiment was calculated using Equation 1 [80].

$$
\mu=\ln \frac{N_{2}-N_{1}}{t_{2}-t_{1}}
$$

Equation 1: Specific growth rate $(\mu)$, where $\mathrm{N}_{1}$ and $\mathrm{N}_{2}$ are the dry biomass values at time $1\left(\mathrm{t}_{1}\right)$ and 2 $\left(\mathrm{t}_{2}\right)$ respectively. 


\subsection{Analysis of metabolites using gas chromatography-mass spectrometry (GC-MS)}

\subsubsection{Evaluation of intracellular metabolite extraction techniques}

\subsubsection{C. fritschii growth and harvest}

$1 \mathrm{~mL}$ of culture (at $\mathrm{OD}_{750 \mathrm{~nm}}=1$ ) was harvested by centrifugation at $2500 \mathrm{rpm}$ for 5 min. Supernatant was removed and cell pellets were extracted immediately.

\subsubsection{Extraction of intracellular metabolites}

Three different mechanical disruption procedures (sonication, ice bath and freezethaw) were coupled with three solvent systems; methanol $(\mathrm{MeOH})$ chloroform $\left(\mathrm{CHCl}_{3}\right)$ and a combination of the two $\left(1: 1, \mathrm{MeOH}: \mathrm{CHCHl}_{3}\right)$ were tested for the extraction of intracellular metabolites from $C$. fritschii. Cell pellets $(\mathrm{n}=2)$ were resuspended in $1 \mathrm{~mL}$ cold $\mathrm{MeOH}, \mathrm{CHCl}_{3}$ or $\mathrm{MeOH}: \mathrm{CHCl}_{3}$ and placed in an ice-bath for $30 \mathrm{~min}$, sonicated for $30 \mathrm{~min}$ or frozen at $-80{ }^{\circ} \mathrm{C}$ and subjected to three freeze-thaw cycle. After extraction, cells were centrifuged at $2500 \mathrm{rpm}$ for 5 minutes. $200 \mu \mathrm{L}$ of each extract was aliquoted and evaporated to dryness using a rotary vacuum concentrator and derivatised as below (Section 1.3.3).

\subsubsection{Experimental $C$. fritschii sample preparation}

Polar and non-polar metabolites were extracted from dried cell pellets for GC-MS analysis from optimised extraction protocol above (Section 1.3.1.2). Briefly a known weight of dried biomass was re-suspended in $\mathrm{MeOH}: \mathrm{CHCl}_{3}: \mathrm{H}_{2} \mathrm{O}(1 \mathrm{~mL}, 2: 2: 1)$ and sonicated using a sonicator probe (Fisher Scientific, product no: FB50) using 6 cycles of $20 \mathrm{~s}$ pulses at $40 \mathrm{~Hz}$ at $0{ }^{\circ} \mathrm{C}$. After centrifugation ( $5 \mathrm{~min}$ at $12000 \mathrm{rpm}$ ), $100 \mu \mathrm{L}$ of each solvent layer (both methanolic and chloroform layers) was aliquoted into new Eppendorf's and evaporate to dryness using a rotary vacuum concentrator (Eppendorf concentrator 5301) and derivatisation as below.

Dried supernatant (where applicable) was re-suspended in $1 \mathrm{~mL}$ of methanol and centrifuged (4000 rpm, $5 \mathrm{~min}$ ). Two hundred $\mu \mathrm{L}$ was aliquoted into new Eppendorf's followed by evaporation to dryness and derivatisation as below.

\subsubsection{Sample derivatisation}

To each $200 \mu \mathrm{L}$ dried sample, $30 \mu \mathrm{L}$ of methoxyamine hydrochloride $(23 \mathrm{mg})$ in pyridine $(1.5 \mathrm{~mL})$ was added and samples were heated at $70{ }^{\circ} \mathrm{C}$ for $45 \mathrm{~min}$. Once 
cooled to room temperature, MSTFA $+1 \%$ TMCS $\left(50 \mu \mathrm{L}\right.$, Thermo Scientific ${ }^{\mathrm{TM}}$, product no: TS-48915) was added and samples heated for an additional $90 \mathrm{~min}$ at 40 ${ }^{\circ} \mathrm{C}$. Once cooled to room temperature, $10 \mu \mathrm{L}$ of tetracosane dissolved in hexane $(2 \mathrm{mg}$ $\mathrm{mL}^{-1}$ ) was added as an internal standard. Derivatised samples were transferred into auto-sample vials ready for analysis.

\subsubsection{GC-MS analysis}

Derivatised sample ( $1 \mu \mathrm{L})$ was loaded onto an Agilent HP-5MS capillary column (30 $\mathrm{m} \times 0.25 \mathrm{~mm} \times 0.25 \mu \mathrm{m})$ in splitless mode at $250{ }^{\circ} \mathrm{C}$. The $\mathrm{GC}$ was operated at a constant flow of $1 \mathrm{~mL} \mathrm{~min}{ }^{-1}$ helium. The temperature program started at $60{ }^{\circ} \mathrm{C}$ for $1 \mathrm{~min}$, followed by temperature ramping at $10^{\circ} \mathrm{C} \mathrm{min}^{-1}$ to a final temperature of $180{ }^{\circ} \mathrm{C}$, this was followed by a second temperature ramping at $4{ }^{\circ} \mathrm{C} \min ^{-1}$ to a final temperature of $300{ }^{\circ} \mathrm{C}$ and held constant at $300{ }^{\circ} \mathrm{C}$ for $15 \mathrm{~min}$. Data acquisition included a mass range of 50 to 650 and resulted in .D data files for analysis.

Chromatograms were deconvoluted using AMDIS (Automated Mass Spectral Deconvolution and Identification System) followed by alignments using the online portal SpectConnect, http://spectconnect.mit.edu/ [81], before identifying peaks using Golm metabolome database, www.gmd.mpimp-golm.mpg.de/, and the NIST 05 (National Institute of Standards and Technology) library. MetaboAnalyst, www.metaboanalyst.ca/, was used for statistical analysis [82,83].

\subsubsection{GC-MS data processing}

GC-MS data sets need deconvolution of co-eluting compounds, the freely available software AMDIS (Automated Mass Spectral Deconvolution and Identification System) was used to process the chromatograms (.D) and produce .ELU files. Settings for AMDIS were as followed [52]; resolution = medium, sensitivity = medium, shape requirement $=$ medium and component width was 10 . The resulting. ELU files were uploaded to SpectConnect, http://spectconnect.mit.edu/, for alignment and conservative component identification [59,81]. Aligned matrices were further analysed and processed using Excel 2010 (Microsoft, USA). The integrated signal (IS) matrix generated was used for relative quantification of peaks. Triplicate missing data points (within time points) were assumed to be lower than detection limit and replaced with half of the minimum integrated signal within each data set. Data normalisation was carried out using the peak area of the internal standard tetracosane and dry weight 
of each sample (intracellular samples only). All duplicate retention times were removed before further analysis.

\subsubsection{Identification}

Identification of peaks was carried out in AMDIS by analysing each chromatogram using the Golm metabolome database (GMD), www.gmd.mpimp-golm.mpg.de/, as a target library, followed by searching the National Institute of Standards and Technology (NIST) 05 library (Scientific Instrument Service, Inc., NJ, USA) with a match factor of $\geq 60 \%$ [62]. Identifications from GMD and NIST were as TMS/MeOX derivatives and names were converted to non-derivatised form for pathway analysis.

Reports were exported in .xls format from AMDIS with first hit only included for further processing using Excel 2010 (Microsoft, USA). A true hit was considered when two or more biological replicates (within the same time-point) contained the peak. If none of the time points contained 'true hits,' the peaks were removed before further analysis. The peaks were reported belonging to level 2 (putatively annotated compounds) or level 4 (unknown compounds) in accordance with the Metabolomics Standards Initiative [63].

\subsubsection{Pathway analysis}

Each identified peak was manually searched in the Kyoto Encylopedia of Genes and Genomes (KEGG) to find biologically relevant metabolites [84]. KEGG name and pathway information was noted to construct generalised pathway schematics.

\subsubsection{Statistical analysis}

MetaboAnalyst was used for multivariate statistical analysis including principle component analysis (PCA), Analysis of Variance (ANOVA) and hierachial heat-map clustering. Statistical analysis of the IS peak lists (in .csv format) of the time-series data was conducted using the time-series/two-factor module. Multivariate analysis was carried out using each column as a different time point and each row representing a metabolite $($ data type $=$ peak intensity table; study design $=$ time-series only; data format $=$ samples in columns) [83]. Missing data points were uploaded as blanks and replaced with half of the minimum integrated signal within each data set. Peaks were normalised to total sum of peaks, log transformed and mean centred prior to statistical analysis (unless otherwise stated). PCA, a one-way repeated ANOVA $(p<0.05)$ and hierachial heat map clustering was used to evaluate the data. A two sample t-Test with 
equal variance was also used as a univariate statistical tool to evaluate data comparing each treatment time point in Excel unless otherwise stated.

\subsection{Analysis of mycosporine-like amino acids (MAAs) using High Performance Liquid Chromatography (HPLC)}

\subsubsection{Method development}

\subsubsection{Crude MAA sample preparation}

Crude mycosporine-like amino acid (MAA) extracted from dulse was donated by Sarah Twigg and Steve Wilson (Unilever, Colworth) for use in High Performance Liquid Chromatography (HPLC) method development. Crude sample 1 was known to contain shinorine, palythine, porphyra, usijurene and palythene. Crude sample 2 was known to contain shinorine, palythine and porphyra and crude sample 3 contained porphyra and shinorine.

Each extract was re-constituted in $1 \mathrm{~mL}$ of water and absorbance was measured in quartz cuvettes using a UV-visible spectrophotometer between 200-450 nm. Extracts were further diluted until an absorbance of 1 AU was measured.

\subsubsection{Macroalgal sample preparation}

The rhodophyta (red alga) Palmaria palmata ( $P$. palmata) was collected from Langland bay, Wales in June 2017. The biomass was washed with deionised $\mathrm{H}_{2} \mathrm{O}$ to remove sediment and stored at $-80{ }^{\circ} \mathrm{C}$ until analysis.

\subsubsection{Macroalgal MAA extraction}

Three replicate $P$. palmata samples were ground using a pestle and mortar and transferred into a $50 \mathrm{~mL}$ centrifuge tube for extraction. $10 \mathrm{~mL} 50 \%$ aqueous (aq) $\mathrm{MeOH}$ was added and the samples were sonicated on ice using a sonicator probe for 5 $\min$ at $50 \mathrm{~Hz}$. The samples were centrifuged at $4400 \mathrm{rpm}$ for $10 \mathrm{~min}$ and the supernatant removed. The extraction protocol was repeated 3 times and supernatants combined.

$\mathrm{MeOH}$ was removed under vacuum and water was removed by freeze-drying for $24 \mathrm{~h}$. Dried extracts were re-constituted with $90 \%$ Ethanol (EtOH, aq) and left at $-20{ }^{\circ} \mathrm{C}$ overnight. Samples were centrifuged for $5 \mathrm{~min}$ at $4400 \mathrm{rpm}$ and supernatant retained. 
EtOH was removed under vacuum and samples were freeze-dried to remove water. Dried extracts were re-dissolved in $1 \mathrm{~mL}$ of water and absorbance measured in quartz cuvettes using a UV-visible spectrophotometer between 200-450 nm. Samples referred to as crude $P P$.

\subsubsection{HPLC analysis}

Method development was performed using a HP Agilent 1100 HPLC system equipped with a binary pump (G1312A), an autosampler (ALS) injector (G1313A), thermostatted column compartment (G1316A) and diode array detector (DAD, G1315A) connected via an interface module to a computer running ChemStation software. All analyses were carried out at $35^{\circ} \mathrm{C}$ with a flow rate of $0.5 \mathrm{~mL} \mathrm{~min}{ }^{-1}$ and injection volume of $5 \mu \mathrm{L}$. Detection of peaks were made by monitoring absorbance at $310,320,330$ and $360 \mathrm{~nm}$ as well as absorption spectra measured between 200-400 $\mathrm{nm}$ which were recorded and stored within each detected peak.

\subsubsection{Mobile phase optimisation}

The three crude extracts ( $n=5$, technical replicates) were separated firstly using an Alltima Altech polymeric double encapped reverse phase C18 column $(5 \mu \mathrm{m}, 4.6 \mathrm{x}$ $150 \mathrm{~mm}$ ) for mobile phase development. Eluent A was a solution of (1) $0.06 \%$ aqueous trifluoroacetic acid (TFA) (v/v) or (2) $0.01 \%$ aqueous TFA (v/v) or (3) $0.001 \%$ aqueous TFA (v/v) while eluent B was kept constant as $70 \% \mathrm{MeOH}(\mathrm{aq})$ containing $0.054 \%$ TFA (v/v). The optimum gradient used was kept constant as in (Table 4)

\subsubsection{Stationary phase optimisation}

The crude extract 1 only ( $\mathrm{n}=5$, technical replicates) was then separated using six different RP-HPLC columns : (Column A) Alltima ${ }^{\circledR}$ Altech C18 column (5 $\mu$ m, 4.6

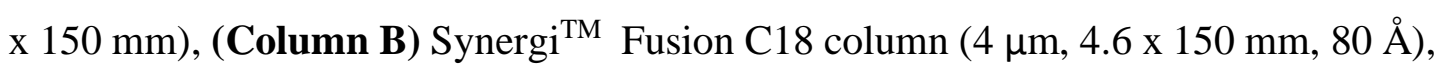
(Column C) Hichrom ACE excel super-C18 column ( $3 \mu \mathrm{m}, 4.6$ x 250 mm, $80 \AA$ ), (Column D) Hichrom ACE excel C18- Amide column (3 $\mu \mathrm{m}, 4.6$ x 250 mm, $100 \AA$ ),

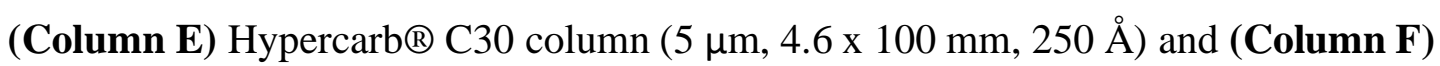

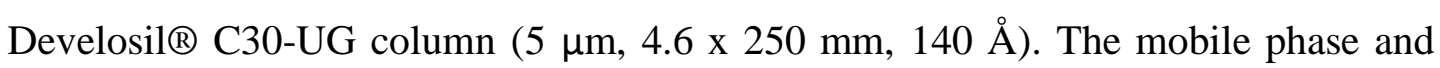
gradient used were as in Section 1.4.1.5 (Mobile phase 2) and Table 4.

Table 4: High-performance liquid chromatography (HPLC) gradient protocol for mycosporine-like amino acid (MAA) separation used throughout method development. 


\begin{tabular}{ccc}
\hline Time (min) & Eluent A (\%) & Eluent B (\%) \\
\hline 0 & 99 & 1 \\
10 & 99 & 1 \\
15 & 80 & 20 \\
20 & 1 & 99 \\
23 & 1 & 99 \\
25 & 99 & 1 \\
30 & 99 & 1 \\
\hline
\end{tabular}

\subsubsection{Data analysis}

For both mobile and stationary phase optimisation, mean values of five replicates and standard error was calculated for each crude extract. Peaks were identified using online absorption spectra and maximum absorbance readings ( $\lambda$ max). Resolution (Rs) between peaks was calculated using Equation 2a, where $\mathrm{RT}_{1}$ and $\mathrm{RT}_{2}$ are the retention times of the two peaks and $\mathrm{Wt}_{1}$ and $\mathrm{Wt}_{2}$ are the widths of those peaks at the baseline [33]. Relative standard deviation (RSD\%) of each detected peak was also calculated to analyse precision and repeatability of each method using Equation $\mathbf{2 b}$, where $\bar{x}$ is the mean peak area and $\sigma$ is the standard deviation of the peak areas of the technical replicates.

$$
\begin{array}{ll}
\mathrm{Rs}=\frac{2(\mathrm{RT} 2-\mathrm{RT} 1)}{\mathrm{Wt} 2+\mathrm{Wt} 1} & (\text { Equation 2a) } \\
\mathrm{RSD} \%=\left(\frac{\sigma}{\bar{x}}\right) \times 100 & \text { (Equation 2b) }
\end{array}
$$

Equation 2: Equations for the (a) calculation of resolution between two peaks and (b) calculation of the relative standard deviation $(\%)$.

\subsubsection{Method validation}

All crude extracts (1,2 and 3$)$ as well as crude $P P$ samples $(\mathrm{n}=3$ biological replicates, $\mathrm{n}=3$ technical replicates) were used to validate the HPLC method as known and 
unknown samples. Extracts were separated using an Alltima Altech polymeric double encapped reverse phase $\mathrm{C} 18$ column $(5 \mu \mathrm{m}, 4.6 \times 150 \mathrm{~mm})$. The mobile phases were $0.01 \%$ aqueous TFA (v/v, eluent $\mathrm{A}$ ) and $70 \% \mathrm{MeOH}$ (aq) containing $0.054 \%$ TFA (v/v, eluent B). The gradient used was as in Table 4.

\subsubsection{C. fritschii MAA extraction}

Each pellet of a known weight was re-suspended in 100\% HPLC grade MeOH (1 mL) and left in the dark at $4{ }^{\circ} \mathrm{C}$ overnight ( $24 \mathrm{~h}$ ). After centrifugation (5 min at $12000 \mathrm{rpm}$ ), the supernatant was removed and evaporated to dryness using a rotary vacuum concentrator. The dried extract was re-dissolved in $600 \mu \mathrm{L}$ of deionised water and transferred to autosample vials for HPLC analysis [78].

\subsubsection{Developed HPLC analysis}

HPLC analysis was performed using an Agilent 1100 system equipped with a binary pump (G1312A), an autosampler injector (ALS, G1313A), thermostatted column compartment (G1316A) and diode array detector (DAD, G1315A) connected via an interface module to a computer running ChemStation software. The stationary phase was an Alltima ${ }^{\mathrm{TM}}$ Altech ${ }^{\mathrm{TM}} \mathrm{C} 18,4.6 \times 150 \mathrm{~mm}, 5 \mu \mathrm{m}$ column heated to $35^{\circ} \mathrm{C}$. The resuspended extracts were injected $(100 \mu \mathrm{L})$ using an auto-sampler. The mobile phases consisted of; Eluent A: Water $(0.01 \%$ TFA, $v / v)$ and Eluent B: $70 \%$ methanol $(0.054 \%$ TFA, $v / v$ ) with a gradient of; $99 \%$ A for $10 \mathrm{~min}$, to $80 \%$ A over $5 \mathrm{~min}$, to $1 \%$ A over $5 \mathrm{~min}$, held for $3 \mathrm{~min}$ and increased to $99 \%$ A over 2 min (Table 4). MAA's were monitored at wavelengths of; 310,320 and $330 \mathrm{~nm}$, absorption spectra between 200 $400 \mathrm{~nm}$ were stored within each detected peak.

\subsubsection{Data analysis}

For each detected MAA peak, retention time, peak area and absorbance at the maximum wavelength $(\lambda \max )$ were collected. The estimated concentration $\left(\mathrm{g} \mathrm{L}^{-1}\right)$ of each MAA was calculated using the Beer-lambert law (Equation 3a, b, c); 


$$
\begin{aligned}
& \mathrm{A}=\text { c. c.l } \\
& \mathrm{c}(\mathrm{M})=\frac{\mathrm{A}}{\varepsilon . l} \\
& \mathrm{c}\left(\mathrm{g} \mathrm{L}^{-1}\right)=\mathrm{c}(\mathrm{M}) \times \mathrm{MW}
\end{aligned}
$$

Equation 3: Estimation of MAA concentration using the Beer-lambert law where; $\mathbf{A}=$ absorbance at $\lambda \max ($ shinorine,$\lambda \max =334 \mathrm{~nm} ; \mathrm{m}$-gly, $\lambda \max =310 \mathrm{~nm}$ ), $\boldsymbol{\varepsilon}=$ extinction coefficient $($ shinorine, $\boldsymbol{\varepsilon}=$ $44700 \mathrm{M}^{-1} \mathrm{~cm}^{-1}$; m-gly, $\left.\boldsymbol{\varepsilon}=28790 \mathbf{M}^{-1} \mathrm{~cm}^{-1}\right)$, $\mathbf{c}=$ concentration, $\mathbf{l}=$ path length and $\mathbf{M W}=$ molecular weight (shinorine, $\mathrm{MW}=332.306 \mathrm{~g} \mathrm{~mol}^{-1} ; \mathrm{m}-\mathrm{gly}, \mathrm{MW}=245.229 \mathrm{~g} \mathrm{~mol}^{-1}$ )

All values were normalised to dry weight of samples $\left(\mathrm{mg} \mathrm{mL}^{-1}\right)$ and expressed as $\mathrm{mg}$ of MAA per mg of dry weight unless otherwise stated. Statistical analysis was carried out in Excel using a two-sample t-Test with equal variance comparing each time point.

\subsection{Pigment analysis}

\subsubsection{Sample preparation for UV-Visible spectroscopy}

To each pellet of a known weight, 100\% HPLC grade MeOH (1 mL) was added and vortexed to re-suspend. Samples were sonicated under low light conditions using a sonicator probe for 6 cycles of $20 \mathrm{~s}$ pulses at $40 \mathrm{~Hz}$ at $0{ }^{\circ} \mathrm{C}$. After centrifugation $(5 \mathrm{~min}$ at $12000 \mathrm{rpm}$ ) the supernatant was removed and absorbance spectra measured using a UV-visible spectrophotometer between $400-800 \mathrm{~nm}$ with $100 \% \mathrm{MeOH}$ as a blank. Chlorophyll (Chl) $a$ and carotenoid concentrations were calculated using the equations as described in Equation 4a and b [85,86].

$$
\begin{gathered}
\operatorname{chl} a\left(\mu g m L^{-1}\right)=13.43 \times A_{665} \times \frac{v}{l V} \quad(\mathbf{4 a}) \\
\text { Total carotenoids }\left(\mu g m L^{-1}\right)=\frac{1000 \times A_{470}-44.76 \times A_{666}}{221} \times \frac{v}{l V}
\end{gathered}
$$

Equation 4: Equation for a) chl $a$ and b) total carotenoids concentrations. $\mathrm{A}_{(\mathrm{XXX})}=$ Absorbance at 665 $\mathrm{nm}, 470 \mathrm{~nm}$ or $666 \mathrm{~nm} . \mathrm{v}=$ volume of $\mathrm{MeOH}, \mathrm{l}=$ path length $(1 \mathrm{~cm})$ and $\mathrm{V}=$ volume of sample. 


\subsection{Total protein analysis}

Total protein analysis was measured using a modified version Lowry protein assay using bovine serum albumin (BSA) as a standard [87,88]. All measurements were carried out in triplicate.

\subsubsection{Preparation of reagents standards}

Lowry A $\left(2 \%(\mathrm{w} / \mathrm{v})\right.$ anhydrous sodium carbonate, $\mathrm{Na}_{2} \mathrm{CO}_{3}$, in $0.1 \mathrm{M}$ sodium hydroxide, $\mathrm{NaOH}$ ) was prepared by dissolving $2 \mathrm{~g}$ of $\mathrm{Na}_{2} \mathrm{CO}_{3}$ in $0.1 \mathrm{M} \mathrm{NaOH}$ (aq) (4 $\mathrm{g}$ of $\mathrm{NaOH}$ in $1 \mathrm{~L} \mathrm{H}_{2} \mathrm{O}$ ). Lowry $\mathrm{B}$ consisted of $1 \mathrm{~g}$ potassium sodium tartrate tetrahydrate, $\mathrm{KNaC}_{4} \mathrm{H}_{4} \mathrm{O}_{6} .4 \mathrm{H}_{2} \mathrm{O}$, in $100 \mathrm{~mL}$ of $\mathrm{H}_{2} \mathrm{O}\left(1 \%\right.$ (w/v) $\mathrm{KNaC}_{4} \mathrm{H}_{4} \mathrm{O}_{6} .4 \mathrm{H}_{2} \mathrm{O}$ in $\mathrm{H}_{2} \mathrm{O}$ ) and Lowry $\mathrm{C}$ was $0.5 \mathrm{~g}$ of copper sulfate pentahydrate, $\mathrm{CuSO}_{4} .5 \mathrm{H}_{2} \mathrm{O}$, in $100 \mathrm{~mL}$ $\mathrm{H}_{2} \mathrm{O}\left(0.5 \%\right.$ (w/v) $\mathrm{CuSO}_{4} .5 \mathrm{H}_{2} \mathrm{O}$ in $\left.\mathrm{H}_{2} \mathrm{O}\right)$. Lowry D was then prepared on the day of amalysis by combining Lowry A, B and C in the ratio of 48:1:1 respectively and the solution was incubated for $10 \mathrm{~min}$ before use. $1 \mathrm{M}$ Folin-Ciocalteu's phenol reagent was prepared by diluting a $2 \mathrm{M}$ solution (Sigma-Aldrich, product no.F9525) with $\mathrm{H}_{2} \mathrm{O}$ in ratio of 1:1 on the day of assay. $24 \%$ Trichloroacetic acid (TCA) was prepared by adding $2.4 \mathrm{~g}$ of TCA to $10 \mathrm{~mL}$ of $\mathrm{H}_{2} \mathrm{O}$. BSA stock solution was prepared by dissolving $10 \mathrm{mg}$ of BSA in $10 \mathrm{~mL}$ of $\mathrm{H}_{2} \mathrm{O}$ to a final concentration of $1 \mathrm{mg} \mathrm{mL}^{-1}$.

\subsubsection{BSA standard curve}

Standard BSA concentrations of $1,0.8,0.6,0.4,0.2,0.1,0.08,0.06,0.04,0.02$ and 0 $\mathrm{mg} \mathrm{mL} \mathrm{m}^{-1}$ were prepared using the table below (Table 5)

BSA standard concentrations $(50 \mu \mathrm{L})$ were added to new $1.5 \mathrm{~mL}$ Eppendorf's along with Lowry D solution $(950 \mu \mathrm{L})$ in triplicate. Samples were vortexed for $30 \mathrm{~s}$ and incubated for $10 \mathrm{~min}$ at room temperature. $1 \mathrm{M}$ Folin-Ciocalteu's phenol reagent $(0.1$ $\mathrm{mL}$ ) was added and samples vortexed immediately to mix. Samples were incubated for a further $30 \mathrm{~min}$ at room temperature and absorbance read at $750 \mathrm{~nm}$ using a UVvisible spectrophotometer. A plot of OD at $750 \mathrm{~nm}$ vs. standard concentration was created using Excel (Figure 5). 
Table 5: Dilution series for BSA standard curve.

\begin{tabular}{ccc}
\hline Standard $\left(\mathrm{mg} \mathrm{mL}^{-1}\right)$ & Volume Stock $(\mathrm{mL})$ & Volume $\mathrm{H}_{2} \mathrm{O}(\mathrm{mL})$ \\
\hline 1 & 1 & 0 \\
0.8 & 0.8 & 0.2 \\
0.6 & 0.6 & 0.4 \\
0.4 & 0.4 & 0.6 \\
0.2 & 0.2 & 0.8 \\
0.1 & 0.1 & 0.9 \\
0.08 & 0.08 & 0.92 \\
0.06 & 0.06 & 0.94 \\
0.04 & 0.04 & 0.96 \\
0.02 & 0.02 & 0.98 \\
0 & 0 & 1 \\
\hline
\end{tabular}

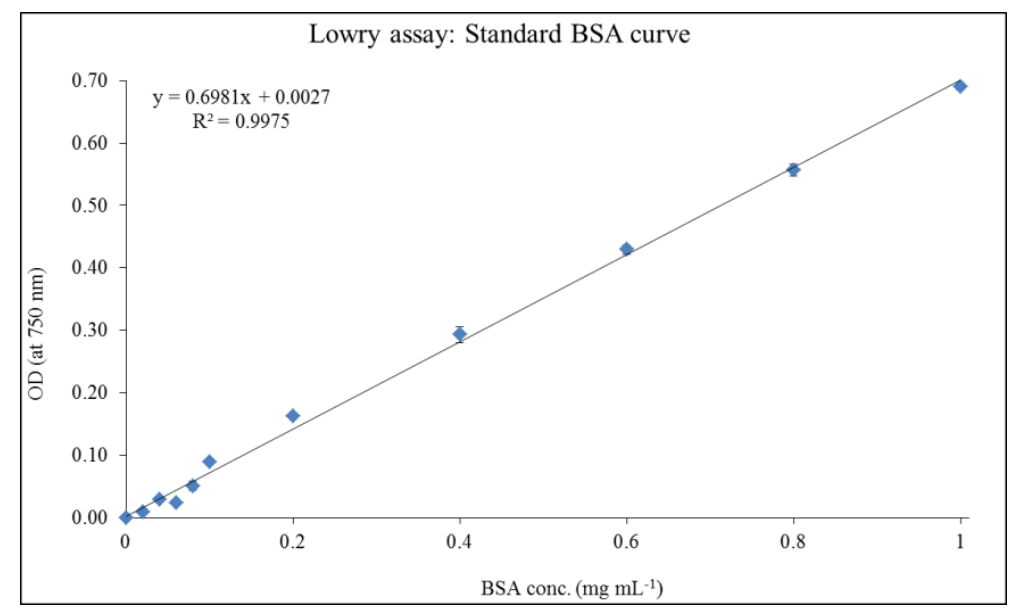

Figure 5: Lowry assay standard curve using BSA. Straight line equation: $y=0.6981 x+0.0027, R^{2}=$ 0.9975 .

\subsubsection{C. fritschii sample analysis}

Dry C. fritschii biomass of a known weight was re-suspended in TCA $(200 \mu \mathrm{L}, 24 \%$ $(\mathrm{w} / \mathrm{v}))$ and incubated at $95^{\circ} \mathrm{C}$ for $15 \mathrm{~min}$. Once cooled, the suspension was diluted to $6 \%$ (w/v) TCA by adding $600 \mu \mathrm{L}$ of $\mathrm{H}_{2} \mathrm{O}$. Samples were centrifuged (5500 rpm, $5 \mathrm{~min}$ ) 
and the supernatant was discarded. The remaining pellets were re-suspended in Lowry $\mathrm{D}(500 \mu \mathrm{L})$ with mixing and incubation at $55^{\circ} \mathrm{C}$. Samples centrifuged $(5500 \mathrm{rpm}, 5$ min) and supernatant retained for analysis.

C. fritschii protein extracts $(50 \mu \mathrm{L})$ were added to new $1.5 \mathrm{~mL}$ Eppendorf's along with Lowry D reagent $(950 \mu \mathrm{L})$. Samples were vortexed for $30 \mathrm{~s}$ and incubated for $10 \mathrm{~min}$ at room temperature. $1 \mathrm{M}$ Folin-Ciocalteu's phenol reagent $(0.1 \mathrm{~mL})$ was added and samples vortexed immediately to mix. Samples were incubated for a further $30 \mathrm{~min}$ at room temperature and absorbance read at $750 \mathrm{~nm}$ using a UV-visible spectrophotometer. Concentration was calculated using straight line equation above followed by data normalisation using dry weight $\left(\mathrm{mg} \mathrm{mL}^{-1}\right)$ for final protein concentration reported as $\mathrm{mg} / \mathrm{mg}$ dry weight.

\subsection{Total carbohydrate analysis}

Total carbohydrates within $C$. fritschii cells were analysed using the colourimetric phenol-sulfuric acid method where simple sugars, oligosaccharides, polysaccharides and derivatives are hydrolysed using $1 \mathrm{M}$ sulfuric acid $\left(\mathrm{H}_{2} \mathrm{SO}_{4}\right)$ followed by dehydration using concentrated $\mathrm{H}_{2} \mathrm{SO}_{4}$ which then react with phenol to produce a yellow colour change $[89,90]$.

\subsubsection{Preparation of reagents and standards}

A 5\% phenol solution was prepared by dissolving $5 \mathrm{~g}$ of phenol in $100 \mathrm{~mL}$ of deionised water. $1 \mathrm{M} \mathrm{H}_{2} \mathrm{SO}_{4}$ (aq) was prepared by adding $55.6 \mathrm{~mL}\left(98 \% \mathrm{H}_{2} \mathrm{SO}_{4}\right)$ to $944.4 \mathrm{~mL}$ of deionised water (total volume $1 \mathrm{~L}$ ). $1 \mathrm{mg} \mathrm{mL}^{-1}$ of glucose stock solution was prepared by adding $100 \mathrm{mg}$ of glucose to $100 \mathrm{~mL}$ of deionised water.

\subsubsection{Glucose standard curve}

Standard glucose concentrations of $1,0.8,0.6,0.4,0.2,0.1,0.08,0.06,0.04,0.02$ and $0 \mathrm{mg} \mathrm{mL}^{-1}$ were prepared using the table below (Table 6).

$0.1 \mathrm{~mL}$ of each glucose concentration (in triplicate) was added to $2 \mathrm{~mL}$ Eppendorf's followed by addition of $1 \mathrm{~mL}$ conc. $\mathrm{H}_{2} \mathrm{SO}_{4}$ and rapid addition of $5 \%$ phenol to surface of liquid for good mixing. Once cool, colour change was measured in acid resistant 
cuvettes at $485 \mathrm{~nm}$ using a UV-visible spectrophotometer. A plot of OD at $485 \mathrm{~nm}$ vs. standard concentration was created using Excel (Figure 6).

Table 6: Dilution series for glucose standard.

\begin{tabular}{ccc}
\hline Standard $\left(\mathrm{mg} \mathrm{mL}^{-1}\right)$ & Volume Stock $(\mathrm{mL})$ & Volume $\mathrm{H}_{2} \mathrm{O}(\mathrm{mL})$ \\
\hline 1 & 1 & 0 \\
0.8 & 0.8 & 0.2 \\
0.6 & 0.6 & 0.4 \\
0.4 & 0.4 & 0.6 \\
0.2 & 0.2 & 0.8 \\
0.1 & 0.1 & 0.9 \\
0.08 & 0.08 & 0.92 \\
0.06 & 0.06 & 0.94 \\
0.04 & 0.04 & 0.96 \\
0.02 & 0.02 & 0.98 \\
0 & 0 & 1 \\
\hline
\end{tabular}

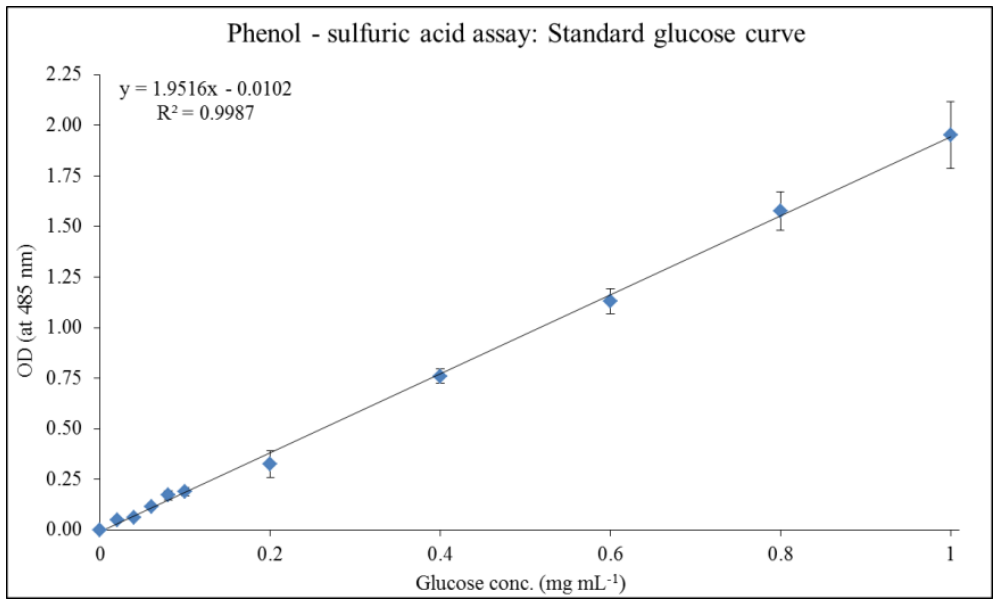

Figure 6: Phenol-sulfuric acid assay standard curve using glucose. Straight line equation: $y=1.9516 x-$ $0.0102, \mathrm{R}^{2}=0.9987$. 


\subsubsection{C. fritschii sample analysis}

To each dry weight pellet, $0.5 \mathrm{~mL}$ of $1 \mathrm{M} \mathrm{H}_{2} \mathrm{SO}_{4}$ was added and heated to $90{ }^{\circ} \mathrm{C}$ for 1 h. Once cooled samples were centrifuged (5500 rpm, $10 \mathrm{~min}$ ) and $0.1 \mathrm{~mL}$ was added to a new Eppendorf. $1 \mathrm{~mL}$ conc. $\mathrm{H}_{2} \mathrm{SO}_{4}$ was added followed by rapid addition of $5 \%$ phenol to surface of liquid for good mixing. Once cool, colour change was measured in acid resistant cuvettes at $485 \mathrm{~nm}$ using a UV-visible spectrophotometer. Concentration was calculated using straight line equation above and data was normalised using dry weight $\left(\mathrm{mg} \mathrm{mL}^{-1}\right)$ for final carbohydrate concentration reported as $\mathrm{mg} / \mathrm{mg}$ dry weight.

\subsection{Anti-inflammatory and Antibacterial activity assessment of $C$. fritschii extracts}

\subsubsection{Cultivation of $C$. fritschii under industrial conditions}

C. fritschii was pre-cultivated in autoclaved deionised water $\left(20 \mathrm{~min}\right.$ at $121{ }^{\circ} \mathrm{C}$ ) from $50 \mathrm{~mL}$ to $1 \mathrm{~L}$ flasks using BG-11 media at $27{ }^{\circ} \mathrm{C} \pm 2{ }^{\circ} \mathrm{C}$ with continuous light at 15 $\mu \mathrm{mol} \mathrm{m} \mathrm{s}^{-1}$ and shaking at $100 \mathrm{rpm}$. Once a dense $1 \mathrm{~L}$ culture was obtained, up-scaling and acclimatisation to industrial conditions was required.

Conditioning of indoor cultures was carried out in the Centre for Sustainable Aquatic Research (CSAR). A $1 \mathrm{~L}$ culture was used to inoculate $4 \mathrm{~L}$ of autoclaved tap water with $50 \mathrm{~mL}$ of BG-11 in a $20 \mathrm{~L}$ carboy. Once dense, $5 \mathrm{~L}$ of filtered tap water and standard $\mathrm{F} / 2$ commercial media $\left(3.5 \mathrm{~mL} \mathrm{~L}^{-1}\right.$ of $\mathrm{H}_{2} \mathrm{O}$, Cell-Hi F2P, Varicon Aqua Solutions, UK) was added and cultures were left to grow until an OD of 0.9-1 was achieved. Once this density was reached, the final $10 \mathrm{~L}$ of tap water was added, which was sterilised by adding $10 \mathrm{~mL}$ of sodium hypochlorite and left running overnight. The sodium hypochlorite was neutralised using sodium thiosulfate $\left(0.3 \mathrm{~g} \mathrm{~L}^{-1}\right.$ of treated water). The sterilised water was added to the carboy along with F/2 media. This culture was left to grow until dry weight of $1.2 \mathrm{~g} \mathrm{~L}^{-1}$ was achieved. Fresh F/2 media was added every 5 days to maintain nutrient levels. The abiotic parameters were a temperature of $20{ }^{\circ} \mathrm{C} \pm 1{ }^{\circ} \mathrm{C}$, aeration was achieved with filtered $(0.2 \mu \mathrm{m})$ ambient air $\left(0.039 \% \mathrm{CO}_{2}\right)$ at a rate of $0.6 \mathrm{~min}^{-1}(\mathrm{v} / \mathrm{v})$ using a $1 \mathrm{~mm}$ capillary glass tube inside the carboy and a light/dark cycle of $18 / 6 \mathrm{~h}$ with cool white fluorescent tubes at $60 \mu \mathrm{mol} \mathrm{m} \mathrm{m}^{-2} \mathrm{~s}^{-1}$, pH was measured every other day and remained at 7-8 throughout growth. 


\subsubsection{Harvest of $20 \mathrm{~L}$ carboy}

After 3 months of growth, $17 \mathrm{~L}$ of culture was harvested and concentrated to $2 \mathrm{~L}$ using a crossflow membrane filtration system (Membranology, UK). The $2 \mathrm{~L}$ of culture was washed thoroughly with deionised water and freeze-dried yielding $20 \mathrm{~g}$ of dry weight biomass.

\subsubsection{UV-B exposure}

$3 \times 1 \mathrm{~L}$ of the $20 \mathrm{~L}$ culture at OD of 1 was harvested and transferred into Quartz flasks (H. Baumbach \& Co.LTD, Suffolk, UK) for UV-B experiments. Cultures were exposed to $24 \mathrm{~h}$ of UV-B at an intensity of $3 \mu \mathrm{mol} \mathrm{m} \mathrm{m}^{-2} \mathrm{~s}^{-1}$ and continuous white light at $15 \mu \mathrm{mol} \mathrm{m} \mathrm{m}^{-2}$ and a temperature of $27^{\circ} \mathrm{C} \pm 2{ }^{\circ} \mathrm{C}$ with continuous shaking at 100 rpm. Cultures were harvested via centrifugation and freeze-dried for $48 \mathrm{~h}$ to yield approx. $1 \mathrm{~g}$ of biomass for extraction.

\subsubsection{Extraction of dried biomass for analysis}

Both UV-B treated ( $3 \times 1 \mathrm{~g})$ and control (no UV-B) (4 x $2 \mathrm{~g}$ ) biomass was extracted using sequential solvent extraction. $20 \mathrm{~mL}$ of hexane (hex) was added to each $50 \mathrm{~mL}$ Falcon tube containing weighed biomass. Cells were sonicated for $10 \mathrm{~min}$ on ice to extract non-polar metabolites into the solvent. The suspension was centrifuged (20 $\left.\min , 8000 \mathrm{rpm}, 4^{\circ} \mathrm{C}\right)$ and the hex supernatant was removed and filtered $(0.2 \mu \mathrm{m})$ into a new Falcon tube, $200 \mu \mathrm{L}$ was used for GC-MS analysis as described above (Section 1.3). The solvent was removed using a vacuum concentrator by drying in aliquots of 2 $\mathrm{mL}$ and combining all extracts to a single Eppendorf. This process was repeated for ethyl acetate (EtOAc), ethanol (EtOH) and $\mathrm{MeOH}$.

\subsubsection{Extract preparation}

All extracts were re-suspended in 100\% dimethylsulfoxide (DMSO) to a final concentration of $10 \mathrm{mg} \mathrm{mL}^{-1}$. Extracts were also combined to form pooled samples of UV-B and control extracts for analysis in anti-inflammatory and antibacterial assays.

\subsubsection{Heterologous expression of mycosporine-glycine in Escherichia coli}

Conducted by Steve Wilson at Unilever, Colworth

The bacterium Escherichia coli (E. coli) was genetically modified to include the Myl synthase gene cluster for the biosynthesis of mycosporine-glycine (m-gly). A starter culture of the expression strain was grown overnight (approx. 20 h) in M9 minimal 
medium at $37{ }^{\circ} \mathrm{C}$ and continuous shaking. This starter culture was used to inoculate a total of $2 \mathrm{~L}$ of M9 minimal media supplemented with glycerol (20 g), kanamycin (50

$\left.\mu \mathrm{g} \mathrm{mL}{ }^{-1}\right)$ and chloramphenicol $\left(34 \mu \mathrm{g} \mathrm{mL}^{-1}\right)$. The cultures were incubated at $37{ }^{\circ} \mathrm{C}$ with continuous shaking until an optical density at $600 \mathrm{~nm}\left(\mathrm{OD}_{600}\right)$ between 0.5 and 0.7 was reached (approx. $4 \mathrm{~h}$ ). The culture was cooled to room temperature by placing on ice and isopropyl- $\beta-\mathrm{D}$ thiogalactopyranoside (IPTG) was added and cultures incubated with shaking at $25^{\circ} \mathrm{C}$ for $24 \mathrm{~h}$.

$2 \mathrm{~L}$ cell culture was harvested by centrifugation $\left(5000 \mathrm{rcf}, 20 \mathrm{~min}\right.$ at $4{ }^{\circ} \mathrm{C}$ ) and supernatant was collected followed by filtration. The filtered supernatant was clarified using a cross-flow membrane filtration system to remove proteins. $20 \mathrm{~g}$ of activated charcoal was added and gently mixed for $10 \mathrm{~min}$. The suspension was filtered (supernatant kept) and washed with $50 \% \mathrm{MeOH}$. UV-visible spectrum of supernatant fraction was collected and absorbance at $310 \mathrm{~nm}$ was noted as m-gly. Fractions with $\mathrm{m}$-gly present were further purified by ion exchange chromatography, acidifying with $\mathrm{HCl}$ and eluting with $\mathrm{H}_{2} \mathrm{O}$. Fractions were freeze-dried to yield partially purified (16\%) m-gly.

\subsubsection{M-gly extract preparation}

$891 \mu \mathrm{L}$ of $\mathrm{H}_{2} \mathrm{O}$ was added to the partially pure m-gly to make up a $2 \mathrm{mM}$ stock solution. This was further diluted with DMEM (10\% FBS) to make up concentrations of 50, 25, 12.5, 6.25, 3.125 and $0 \mu \mathrm{M}$ for use in anti-inflammatory assays (Table 12,

\section{Section 1.8.3).}

\subsubsection{Mammalian Cell Culture for in vitro anti-inflammatory assessment}

\subsubsection{Cell line, media composition and growth conditions}

Immortalised human keratinocyte (HaCaT) cells were donated by Dr Mark Fowler (Unilever, UK). The cells were maintained in a T175 flasks with approximately $2 \times 10^{6}$ cells per flask containing $25 \mathrm{~mL}$ of Dulbecco's Minimum Eagle Medium (DMEM) with GlutaMAX ${ }^{\mathrm{TM}}\left(\mathrm{Gibco}^{\mathrm{TM}}\right.$, product no. 31966021) supplemented with heat inactivated $10 \%$ Fetal Bovine Serum (FBS, Gibco ${ }^{\mathrm{TM}}$, product no. A3160802). The flasks were incubated in $5 \% \mathrm{CO}_{2}$ atmosphere in a humidified incubator at $37{ }^{\circ} \mathrm{C}$ for approximately 3-4 days. 


\subsubsection{Maintenance of cell culture}

The cells were split once a confluency of $80 \%$ was reached. Briefly, the cells washed thoroughly with $10 \mathrm{~mL}$ Dulbecco's Phosphate Buffered Saline (dPBS, Sigma Aldrich, product no: D8537). The dPBS was aspirated and $4 \mathrm{~mL}$ of TrypLE ${ }^{\mathrm{TM}}$ Express $\left(\right.$ Gibco $^{\mathrm{TM}}$, product no: 12604013$)$ was added and cells incubated for $8 \mathrm{~min}$ to detach the cells (tryptanization). $8 \mathrm{~mL}$ fresh media was added to re-suspend the trytanized cells. The volume of cell suspension was measured (approx. $12 \mathrm{~mL}$ ) and added to a universal tube.

$100 \mu \mathrm{L}$ of the cell suspension was removed from the universal and added to $9.9 \mathrm{~mL}$ of ISOTON® II solution for cell counts (Z-series Coulter Counter, Beckman Coulter, Inc, US). Cell numbers were counted twice (blanks of ISOTON® II only were run before and after cell counts) and total cell number per flask was calculated using Equation $\mathbf{5 a}$ and $\mathbf{5 b}$ below;

$($ Cell count $1+$ Cell count 2$) \times 100=$ Cells $m L^{-1}$

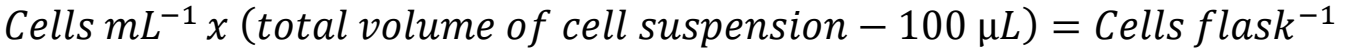

Equation 5: Equations for the calculation of cell numbers (a) per mL of culture and (b) total per flask.

The cell suspension was centrifuged (1000 $\mathrm{rcf}, 5 \mathrm{~min}$ ) and the old medium was aspirated. The remaining cell pellet was re-suspended in the equivalent amount of fresh medium to make up a suspension of $1 \times 10^{6}$ cells $\mathrm{mL}^{-1}$ (e.g. $8.5 \mathrm{~mL}$ of medium added to $8.5 \times 10^{6}$ total cells to make $1 \times 10^{6}$ cells $\mathrm{mL}^{-1}$ ). $23 \mathrm{~mL}$ of fresh medium was added into new T175 flasks and $2 \mathrm{~mL}$ of the $1 \times 10^{6}$ cells $\mathrm{mL}^{-1}$ suspension was added to a final concentration of $2 \times 10^{6}$ cells $\mathrm{mL}^{-1}$. Flasks were incubated at $37{ }^{\circ} \mathrm{C}$ in $5 \% \mathrm{CO}_{2}$ atmosphere in a humidified incubator until confluent (3-4 days).

\subsubsection{Anti-inflammatory assay}

\subsection{Seeding density experiment}

For the HaCaT cell seeding density experiment, a dilution series of cell concentrations was prepared as below (

Table 7). Cells were tryptanized using TrypLE ${ }^{\mathrm{TM}}$ Express and cells counted as above (Section 1.8.3.2). The cell suspension in the universal tube was centrifuged (1000 rcf, 
$5 \mathrm{~min}$ ) and the old medium was aspirated (removing bubbles first then slowly aspirating the medium). The cell pellet was agitated by tapping the tube to prevent clumping during re-suspension. Cells were diluted to 1 x $10^{6}$ cells $\mathrm{mL}^{-1}$ (e.g. $9.9 \mathrm{~mL}$ of fresh medium added to $9.9 \times 10^{6}$ cells) and pipetted repeatedly to re-suspend the cells homogeneously; this dilution was known as cell stock $\mathbf{A}$.

The dilution of cell stock $\mathbf{A}$ to the working stocks of $1 \times 10^{5}, 5 \times 10^{4}, 1 \times 10^{4}, 5 \times 10^{3}$ and $1 \times 10^{3}$ cells $\mathrm{mL}^{-1}$ was calculated as below (

\section{Table 7).}

Each dilution was added to a $24\left(1 \mathrm{~mL} \mathrm{well}^{-1}\right)$ and an E8 iCELLigence plate $(320 \mu \mathrm{L}$ well ${ }^{-1}$ ). Plates were rested for $15 \mathrm{~min}$ before placing in an incubator for $48 \mathrm{~h}$ at $37{ }^{\circ} \mathrm{C}$ in $5 \% \mathrm{CO}_{2}$ atmosphere in a humidified incubator. Cell density was monitored using a microscope and iCELLigence real time measurements (Section 1.8.4).

Table 7: Dilution series for dose seeding experiment

\begin{tabular}{ccccc}
\hline Dilution & Cells mL & DMEM $(\mathrm{mL})$ & Stock $(\mathrm{mL})$ & Stock/dilution \\
\hline Stock A & $1 \times 10^{6}$ & 3.5 & - & $\begin{array}{c}\text { Re-suspended } \\
\text { cell pellet }\end{array}$ \\
A & $1 \times 10^{5}$ & 9 & 1 & Stock A \\
B & $5 \times 10^{4}$ & 9.5 & 0.5 & Stock A \\
C & $1 \times 10^{4}$ & 9 & 1 & A \\
D & $5 \times 10^{3}$ & 9 & 1 & B \\
E & $1 \times 10^{3}$ & 9 & 1 & C \\
\hline
\end{tabular}

\subsection{Cell seeding}

For the dose response experiments, cells were tryptanized using TrypLE ${ }^{\mathrm{TM}}$ Express and cells counted as above (Section 1.8.3.2) to create cell stock $\mathbf{A}$ at $1 \times 10^{6}$ cells $\mathrm{mL}^{-}$ ${ }^{1}$ (Section 1.8.3.3.1). The dilution of cell stock $\mathbf{A}$ to a working stock of 1 x $10^{5}$ cells $\mathrm{mL}^{-1}$ was calculated as followed: 
A total volume of $28 \mathrm{~mL}$ was needed to seed a 24 well plate at $1 \mathrm{~mL}^{-1}$ well $^{-1}$ (plus pipetting error). The total volume of fresh media and volume of cell stock A needed was calculated using Equation $\mathbf{6 a}, \mathbf{b}$ and $\mathbf{c}$ below;

$\frac{\text { Initial cell concentration }\left(1 \times 10^{6} \text { cells } m L^{-1}\right)}{\text { Final cell concentration }\left(1 \times 10^{5} \text { cells } m L^{-1}\right)}=$ Dilution factor $(10)$

$\frac{\text { Total volume }(28 \mathrm{~mL})}{D F(10)}=$ Volume of stock $A$ needed $(2.8 \mathrm{~mL})$

Total volume $(28 \mathrm{~mL})$ - Volume of stock $B(2.8 \mathrm{~mL})=$

Volume of additional media $(25.2 \mathrm{~mL})$

Equation 6: Equations for the dilution of cells to experimental seeding density. (a) Calculation of dilution factor, (b) volume of initial cell volume needed and (c) volume of addition media needed.

$1 \mathrm{~mL}$ of $1 \times 10^{5}$ cells $\mathrm{mL}^{-1}$ was seeded in each well of a 24 -well plate. Plates were left to rest for 15 min followed by incubation at $37{ }^{\circ} \mathrm{C}$ in $5 \% \mathrm{CO}_{2}$ atmosphere in a humidified incubator for 24 - $48 \mathrm{~h}$ until a monolayer of cells had formed.

\subsection{Pro-inflammatory dose response in HaCaT cells}

After $48 \mathrm{~h}$ of $\mathrm{HaCaT}$ cell incubation (Section 1.8.3.3.2), the spent media was aspirated and the cells were washed with $1 \mathrm{~mL}$ of dPBS. $1 \mathrm{~mL}$ of pro-inflammatory mediator stocks, Tumour necrosis factor- $\alpha$ (TNF- $\alpha$ ) and Interleukin-1 $\beta$ (IL-1 $\beta$ ) (Table 8) were added to the corresponding wells $(n=3)$, each stock was vortexed to ensure even mixing. For cell only control samples, mediators were replaced with $\mathrm{H}_{2} \mathrm{O}$ as a vehicle control $(\mathrm{n}=3)$. The plates were incubated for $24 \mathrm{~h}$ in $5 \% \mathrm{CO}_{2}$ atmosphere in a humidified incubator at $37^{\circ} \mathrm{C}$. 
Table 8: Dilution series for TNF- $\alpha$ and IL-1 $\beta$ dose response

\begin{tabular}{cccc}
\hline TNF- $\alpha\left(\mu \mathrm{g} \mathrm{mL}{ }^{-1}\right)$ & TNF- $\alpha$ stock $(\mu \mathrm{L})$ & $\mathrm{H}_{2} \mathrm{O}(\mu \mathrm{L})$ & $\begin{array}{c}\text { Stock used }(\mu \mathrm{g} \\
\left.\mathrm{mL}^{-1}\right)\end{array}$ \\
\hline 10 & 10 & 0 & 10 \\
5 & 5 & 5 & 10 \\
1 & 2 & 18 & 10 \\
0 & 0 & 10 & - \\
\hline $\mathrm{IL}-1 \beta\left(\mu \mathrm{g} \mathrm{mL} \mathrm{L}^{-1}\right)$ & $\mathrm{IL}-1 \beta$ stock $(\mu \mathrm{L})$ & $\mathrm{H}_{2} \mathrm{O}(\mu \mathrm{L})$ & $\begin{array}{c}\text { Stock used }(\mu \mathrm{g} \\
\left.\mathrm{mL}^{-1}\right)\end{array}$ \\
\hline 1 & 10 & 0 & 1 \\
0.5 & 5 & 5 & 1 \\
0.1 & 2 & 18 & - \\
\hline 0 & 0 & 10 & 1 \\
\hline
\end{tabular}

*1:1000 dilution: $3.5 \mu \mathrm{L}$ of TNF- $\alpha / \mathrm{IL}-1 \beta$ stocks or vehicle control into $3.5 \mathrm{~mL}$ DMEM (10\% and $1 \%$ FBS)

\subsection{Pro-inflammatory dose response of TNF- $\alpha$ (10\% FBS in DMEM)}

After $48 \mathrm{~h}$ of HaCaT cell incubation (Section 1.8.3.3.2), the spent media was aspirated and the cells were washed with $1 \mathrm{~mL}$ of dPBS. $1 \mathrm{~mL}$ of pro-inflammatory mediator stocks (Table 9) were added to the corresponding wells $(\mathrm{n}=3$, each stock was vortexed to ensure even mixing). For cell only control samples, mediators were replaced with $\mathrm{H}_{2} \mathrm{O}$ as a vehicle control $(\mathrm{n}=3)$. The plates were incubated for $24 \mathrm{~h}$ in $5 \% \mathrm{CO}_{2}$ atmosphere in a humidified incubator at $37^{\circ} \mathrm{C}$. 
Table 9: Extended dilution series for TNF- $\alpha$ dose response

\begin{tabular}{cccc}
\hline TNF- $\alpha\left(\mu \mathrm{g} \mathrm{mL}^{-1}\right)$ & TNF- $\alpha$ stock $(\mu \mathrm{L})$ & $\mathrm{H}_{2} \mathrm{O}(\mu \mathrm{L})$ & $\begin{array}{c}\text { Stock used } \\
\left(\mu \mathrm{g} \mathrm{mL}^{-1}\right)\end{array}$ \\
\hline $10($ Stock C) & 10 & 0 & 10 \\
5 & 5 & 5 & 10 \\
1 & 2 & 18 & 10 \\
0.5 & 2 & 18 & 5 \\
0.1 & 2 & 18 & 1 \\
0.01 & 2 & 18 & 0.1 \\
$0(\mathrm{VC})$ & 0 & 10 & - \\
\hline
\end{tabular}

${ }^{*}$ 1:1000 dilution: $3.5 \mu \mathrm{L}$ of TNF- $\alpha$ or vehicle control $\left(\mathrm{H}_{2} \mathrm{O}\right)$ into $3.5 \mathrm{~mL}$ DMEM (10\% FBS)

\subsection{Anti-inflammatory dose response of hydrocortisone}

After $48 \mathrm{~h}$ of HaCaT cell incubation (Section 1.8.3.3.2), anti-inflammatory dose response of hydrocortisone was investigated. Spent medium was aspirated and washed with $1 \mathrm{~mL}$ of dPBS. $1 \mathrm{~mL}$ of each hydrocortisone (HC) stock (Table 10) was added to the monolayer of cells $(n=3$, each stock was vortexed to ensure even mixing). For cell only control samples, HC was replaced with DMSO as a vehicle control $(\mathrm{n}=3)$. The plates were incubated for $24 \mathrm{~h}$ at $37{ }^{\circ} \mathrm{C}$ in $5 \% \mathrm{CO}_{2}$ atmosphere in a humidified incubator.

After $24 \mathrm{~h}$, the spent medium was aspirated and cells were washed with $1 \mathrm{~mL}$ of dPBS. Active stock concentrations were freshly prepared as above with the addition of $1 \mathrm{ng}$ $\mathrm{mL}^{-1}$ TNF- $\alpha$ (1:1000 dilution). For cell only control samples, actives were replaced with DMSO and TNF- $\alpha$ was replaced with $\mathrm{H}_{2} \mathrm{O}$ as vehicle controls $(\mathrm{n}=3)$. The plate was incubated for a further $24 \mathrm{~h}$ in $5 \% \mathrm{CO}_{2}$ atmosphere in a humidified incubator at $37^{\circ} \mathrm{C}$. 
Table 10: Dilution series for HC anti-inflammatory dose response

\begin{tabular}{cccc}
\hline $\mathrm{HC}(\mathrm{mM})$ & HC stock $(\mu \mathrm{L})$ & DMSO $(\mu \mathrm{L})$ & Stock used $(\mathrm{mM})$ \\
\hline 10 & 10 & 0 & 10 \\
1 & 2 & 18 & 10 \\
0.1 & 2 & 18 & 1 \\
0.01 & 2 & 18 & 0.1 \\
0.001 & 2 & 18 & 0.01 \\
0.0001 & 2 & 18 & 0.001 \\
$0(\mathrm{VC})$ & 0 & 20 & - \\
\hline
\end{tabular}

*1:1000 dilution: $3.5 \mu \mathrm{L}$ of $\mathrm{HC}$ or vehicle control (DMSO) into $3.5 \mathrm{~mL}$ DMEM (10\% FBS)

\subsection{Anti-inflammatory dose response of pooled C. fritschii extracts}

After $48 \mathrm{~h}$ of $\mathrm{HaCaT}$ cell incubation (Section 1.8.3.3.2), anti-inflammatory dose response of $C$. fritschii pooled extracts (Section 1.8.1.3 and 1.8.1.4) was investigated at concentrations of $10,5,1,0.5,0.1,0.01$ and $0 \mu \mathrm{g} \mathrm{mL}^{-1}$. Spent medium was aspirated and washed with $1 \mathrm{~mL}$ of dPBS. $1 \mathrm{~mL}$ of each $C$. fritschii stock (Table 11) was added to the monolayer of cells with $\mathrm{HC}(10 \mu \mathrm{M})$ as a positive control $(\mathrm{n}=3$, each stock was vortexed to ensure even mixing). For cell only control samples, extracts were replaced with DMSO as a vehicle control $(\mathrm{n}=3)$. The plates were incubated for $24 \mathrm{~h}$ at $37^{\circ} \mathrm{C}$ in $5 \% \mathrm{CO}_{2}$ atmosphere in a humidified incubator.

After $24 \mathrm{~h}$, the spent medium was aspirated and cells were washed with $1 \mathrm{~mL}$ of dPBS. Active stock concentrations were freshly prepared as above with the addition of $1 \mathrm{ng}$ $\mathrm{mL}^{-1}$ TNF- $\alpha$ (1:1000 dilution). For cell only control samples, actives were replaced with DMSO and TNF- $\alpha$ was replaced with $\mathrm{H}_{2} \mathrm{O}$ as vehicle controls in triplicate. The plate was incubated for a further $24 \mathrm{~h}$ in $5 \% \mathrm{CO}_{2}$ atmosphere in a humidified incubator at $37^{\circ} \mathrm{C}$. 
Table 11: Dilution series for dose response of $C$. fritschii extracts

\begin{tabular}{cccc}
\hline $\begin{array}{c}\text { C. fritschii extracts } \\
\left(\mathrm{mg} \mathrm{mL}^{-1}\right)\end{array}$ & $\begin{array}{c}\text { Extract stock } \\
(\mu \mathrm{L})\end{array}$ & DMSO $(\mu \mathrm{L})$ & $\begin{array}{c}\text { Stock used } \\
\left(\mathrm{mg} \mathrm{mL}^{-1}\right)\end{array}$ \\
\hline 10 & 10 & 0 & 10 \\
5 & 5 & 5 & 10 \\
1 & 2 & 18 & 10 \\
0.5 & 5 & 5 & 1 \\
0.1 & 2 & 18 & 0.1 \\
0.01 & 2 & 18 & - \\
$0(\mathrm{VC})$ & 0 & 20 & 1 \\
\hline
\end{tabular}

*1:1000 dilution: $3.5 \mu \mathrm{L}$ of $C$. fritschii extracts or vehicle control (DMSO) into $3.5 \mathrm{~mL}$ DMEM (10\% FBS) to produce final concentration of $10-0 \mu \mathrm{g} \mathrm{mL}^{-1}$.

\subsection{Anti-inflammatory dose response of individual C. fritschii extracts}

The procedure above was repeated with individual $C$. fritschii extracts (Section 1.8.1.3 and 1.8.1.4) at concentrations of $1,0.5 .0 .1$ and $0 \mu \mathrm{g} \mathrm{mL}^{-1}$ with $\mathrm{HC}$ as a control (10

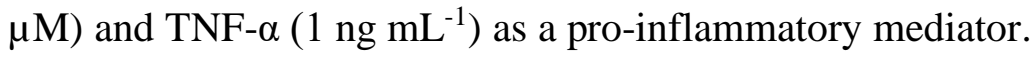

\subsection{Anti-inflammatory dose response of $m$-gly}

The procedure above was repeated with partially pure m-gly (Section 1.8.2) at concentrations of $50,25,12.5,6.25,3.125$ and $0 \mu \mathrm{M}$ (Table 12). $3.5 \mathrm{~mL}$ of each stock was added to the corresponding wells $(\mathrm{n}=3)$ with $\mathrm{HC}$ as a control $(10 \mu \mathrm{M})$ and TNF- $\alpha$ $\left(1 \mathrm{ng} \mathrm{mL} \mathrm{m}^{-1}\right)$ as a pro-inflammatory mediator. 
Table 12: Dilution series for m-gly anti-inflammatory dose response.

\begin{tabular}{cccc}
\hline M-gly $(\mu \mathrm{M})$ & M-gly stock $(\mathrm{mL})$ & DMEM $(\mathrm{mL})$ & Stock used $(\mathrm{mM})$ \\
\hline 50 & 0.2 & 8 & $2 \mathrm{mM}$ \\
25 & 4 & $4 *$ & $50 \mu \mathrm{M}$ \\
12.5 & 4 & $4 *$ & $25 \mu \mathrm{M}$ \\
6.25 & 4 & $4 *$ & $12.5 \mu \mathrm{M}$ \\
3.125 & 4 & $4 *$ & $6.25 \mu \mathrm{M}$ \\
0 & 0 & $4 *$ & - \\
\hline
\end{tabular}

*Vehicle control $=23.4 \mathrm{~mL}$ of DMEM $(10 \% \mathrm{FBS})+600 \mu \mathrm{L}$ of $\mathrm{H}_{2} \mathrm{O}$, to use as diluent to take into account $\mathrm{H}_{2} \mathrm{O}$ added to cells.

\subsection{Supernatant and cell lysate preparation}

After 24 or $48 \mathrm{~h}$ the supernatant of each well was collected and centrifuged in a deepwell 96 microplate (600 rcf, $5 \mathrm{~min}$ ). The cell free supernatant was then aliquoted into two round-bottomed 96 microplates and frozen at $-20{ }^{\circ} \mathrm{C}$ until cytokine analysis. 250 $\mu \mathrm{L}$ of RIPA buffer (Sigma Aldrich, product no: R0278) was added to the remaining cells and the plates were frozen at $-20{ }^{\circ} \mathrm{C}$ until protein analysis.

\subsection{Analysis of secreted inflammatory cytokines using an Enzyme-linked immunosorbent assay (ELISA)}

For cytokine quantification, IL-6 and IL-8 secretion was monitored using enzymelinked immunosorbent assays (Human IL-6 DuoSet® ELISA, DY206 and Human IL-8/CXCL8 DuoSet® ELISA, DY208, R\&D systems, UK). The following procedure was carried out according to the manufacturer's instructions with preparation of IL-6 and IL-8 reagents as in Table 13.

\subsubsection{Plate preparation}

To prepare the 96-well plates for IL-6 and IL-8 analysis, $100 \mu \mathrm{L}$ of capture antibody was added to each well (IL-6 \& IL-8; $58.3 \mu \mathrm{L}$ of stock into $7 \mathrm{~mL}$ of PBS) and the plates were sealed and left at room temperature overnight (approx. $15 \mathrm{~h}$ ). The wells 
were washed (3 x $300 \mu \mathrm{L}$ well $\left.^{-1}\right)$ with wash buffer $\left(0.05 \% \mathrm{TWEEN}^{\circledR}\right.$ in PBS) followed by the addition of $300 \mu \mathrm{L}$ well ${ }^{-1}$ of block buffer (1\% BSA in PBS). The plates were sealed and left for $1 \mathrm{~h}$ at room temperature.

\subsubsection{Assay procedure}

The wells were washed with wash buffer $\left(3 \times 300 \mu \mathrm{L}_{\text {well }}{ }^{-1}\right)$, for IL-6 analysis, 100 $\mu \mathrm{L}$ well ${ }^{-1}$ of sample was added and for IL- 8 analysis, $50 \mu \mathrm{L}$ of sample was diluted with $50 \mu \mathrm{L}$ of IL-8 reagent diluent $\left(0.1 \%\right.$ BSA, $0.05 \%$ TWEEN $\left.^{\circledR}\right)$. IL-6 and IL-8 standards were added to each plate using a two-fold serial dilution (Figure 7). The plates were sealed and incubated at room temperature for $2 \mathrm{~h}$ with shaking at $300 \mathrm{rpm}$. Wells were washed with wash buffer $\left(3 \times 300 \mu \mathrm{L}_{\text {well }}{ }^{-1}\right)$ and $100 \mu \mathrm{L}$ well ${ }^{-1}$ of detection antibody was added (IL-6; $116.67 \mu \mathrm{L}$ in $7 \mathrm{~mL} 1 \%$ BSA and IL-8; $116.67 \mu \mathrm{L}$ in $7 \mathrm{~mL} 0.1 \%$ BSA), the plates were sealed and left shaking at $300 \mathrm{rpm}$ for an additional $2 \mathrm{~h}$ at room temperature. Plates were washed again with wash buffer $\left(3 \times 300 \mu \mathrm{L}\right.$ well $\left.{ }^{-1}\right)$ and 100 $\mu \mathrm{L}$ well ${ }^{-1}$ of Streptavidin-HRP (IL-6; $175 \mu \mathrm{L}$ in $7 \mathrm{~mL} \mathrm{1 \%} \mathrm{BSA} \mathrm{and} \mathrm{IL-8;} 175 \mu \mathrm{L}$ in 7 $\mathrm{mL} 0.1 \%$ BSA) was added, the plates were sealed and incubated in the dark at room temperature for $20 \mathrm{~min}$. Plates were washed with wash buffer $\left(4 \times 300 \mu \mathrm{L}\right.$ well $\left.{ }^{-1}\right)$ and $100 \mu \mathrm{L}$ well ${ }^{-1}$ of the substrate solution was added (1:1 dilution; $7 \mathrm{~mL}$ of colour reagent $\mathrm{A}\left(\mathrm{H}_{2} \mathrm{O}_{2}\right)$ and $7 \mathrm{~mL}$ of colour reagent $\mathrm{B}$ (tetramethylbenzidine)), the plates were sealed and left in the dark for $15 \mathrm{~min}$ (IL-6) or $10 \mathrm{~min}$ (IL-8) for colour development. $50 \mu \mathrm{L}$ well ${ }^{-1}$ of stop solution $\left(1 \mathrm{~N} \mathrm{H}_{2} \mathrm{SO}_{4}\right)$ was added after the specified times and a colour change from blue to yellow was observed. The plates were read immediately at 450 and $540 \mathrm{~nm}$ using a Multiskan ${ }^{\mathrm{TM}}$ FC Multiplate Photometer (Thermo Scientific). 
Table 13: Preparation of IL-6 and IL-8 reagents. IL-6 reagent diluent $=1 \%$ BSA in PBS, IL- 8 reagent diluent $=0.1 \%$ BSA, $0.05 \%$ TWEEN® 20 in Tris-Buffered Saline.

\begin{tabular}{|c|c|c|c|}
\hline Description & $\begin{array}{c}\text { Stock conc. }(\mu \mathrm{g} \\
\left.\mathrm{mL}^{-1}\right)\end{array}$ & $\begin{array}{l}\text { Working conc. } \\
\qquad\left(\mu \mathrm{g} \mathrm{mL}^{-1}\right)\end{array}$ & Dilution \\
\hline \multicolumn{4}{|l|}{ IL-6 } \\
\hline Capture Antibody & 240 & 2 & 1:120 (PBS) \\
\hline Detection Antibody & 3 & 0.05 & $1: 60(1 \%$ BSA $)$ \\
\hline Standard & $180\left(\mathrm{ng} \mathrm{mL}^{-1}\right)$ & $0.6\left(\mathrm{ng} \mathrm{mL}^{-1}\right)$ & $1: 300(1 \% \text { BSA })^{*}$ \\
\hline Streptavidin-HRP & & & $1: 40(1 \%$ BSA $)$ \\
\hline \multicolumn{4}{|l|}{ IL-8 } \\
\hline Capture Antibody & 480 & 4 & 1:120 (PBS) \\
\hline Detection Antibody & 1.2 & 0.02 & $1: 60(0.1 \% \mathrm{BSA})$ \\
\hline Standard & $100\left(\mathrm{ng} \mathrm{mL}^{-1}\right)$ & $2\left(\mathrm{ng} \mathrm{mL} L^{-1}\right)$ & $1: 50(0.1 \% \mathrm{BSA})^{*}$ \\
\hline Streptavidin-HRP & & & $1: 40(0.1 \% \mathrm{BSA})$ \\
\hline
\end{tabular}



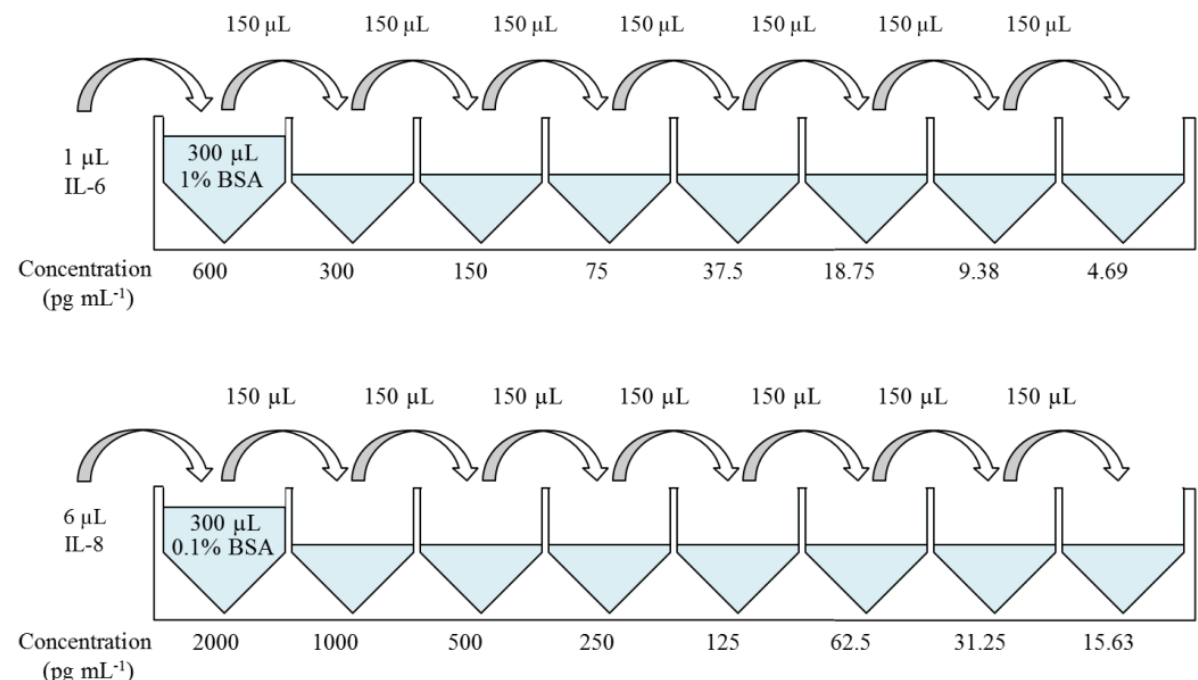

Figure 7: Two-fold dilution series of IL-6 and IL-8 standards. Standards are analysed in duplicate and used to calculate concentration of secreted IL- 6 and IL-8 in samples using quadratic equation of plotted values (concentration vs. absorbance); $\mathrm{y}=\mathrm{ax}^{2}+\mathrm{bx}+\mathrm{c}$.

\subsubsection{ELISA Data analysis}

All absorbance values at $540 \mathrm{~nm}$ were subtracted from absorbance's read at $450 \mathrm{~nm}$. These values were then used to calculate the concentration using the IL- 6 and IL- 8 standard curve quadratic equation. Coefficient of variation $(\% \mathrm{CV})$ was used to determine repeatability of assay using Equation 7;

$$
\% C V=\frac{\sigma}{\mu} \times 100
$$

Equation 7: General formula to calculate coefficient of variation (\%CV) to assess repeatability of assay. Where $\sigma=$ standard deviation and $\mu=$ mean. For ELISA assays a $\% \mathrm{CV} \leq 5$ and for protein assay a $\% \mathrm{CV}$ $\leq 10$ shows good repeatability.

\subsection{Protein concentration assay}

Protein concentration for each well was calculated using the Pierce ${ }^{\mathrm{TM}}$ BCA protein assay (Product no: 23225, Thermo Scientific ${ }^{\mathrm{TM}}$ ). The following procedure was carried out according to the manufacturer's instructions. Frozen cells containing RIPA buffer were thawed and lysate centrifuged in Eppendorf's to remove cell debris. $12.5 \mu \mathrm{L}$ of 
each lysate was added to each well of a 96-well microplate with $12.5 \mu \mathrm{L}$ of PBS. 25 $\mu \mathrm{L}$ of standards (Table 14) were added to the corresponding wells followed by 200 $\mu \mathrm{L}$ well ${ }^{-1}$ of working reagent (A:B, 50:1, $\left.20 \mathrm{~mL} \mathrm{~A}+0.5 \mathrm{~mL} \mathrm{~B}\right)$. The plates were incubated at $37{ }^{\circ} \mathrm{C}$ for $15 \mathrm{~min}$ and absorbance read at $540 \mathrm{~nm}$.

Table 14: BCA standard preparation for protein assay

\begin{tabular}{cccc}
\hline BCA $(\mathrm{mg} / \mathrm{mL})$ & Stock $(\mathrm{mL})$ & $\begin{array}{c}\text { RIPA:PBS* } \\
(50: 50)(\mathrm{mL})\end{array}$ & Stock used \\
\hline 2 & 1 & 0 & 2 \\
1 & 0.5 & 0.5 & 2 \\
0.5 & 0.5 & 0.5 & 1 \\
0.125 & 0.5 & 0.5 & 0.5 \\
0.0625 & 0.5 & 0.5 & 0.125 \\
0 & 0 & 1 & - \\
\hline
\end{tabular}

* Standards are supplied in PBS, need to dilute with RIPA to consider lysate composition (lysed using RIPA).

\subsubsection{Data analysis}

Protein concentration was calculated by using equation of standard curve. $\% \mathrm{CV}$ was used to determine repeatability of assay (Equation 7). IL-6 and IL-8 secretion was normalised to protein data to remove unwanted variation due to cell numbers.

\subsubsection{Cell proliferation assay using real time iCELLigence system}

Proliferation experiments were carried out using the label free real-time cell analyser (RTCA) iCELLigence ${ }^{\mathrm{TM}}$ system (ACEA Biosciences, Inc., USA). Two E8 well plates were incubated prior to analysis for $10 \mathrm{~min}$ in $5 \% \mathrm{CO}_{2}$ atmosphere in a humidified incubator at $37^{\circ} \mathrm{C}$.

Initially $150 \mu \mathrm{L}$ of cell free DMEM supplemented with $10 \%$ FBS was added to the wells and a background reading was recorded. HaCaT cells were harvested (Section 1.8.3.2) and seeded $(300 \mu \mathrm{L})$ at $5 \times 10^{3}, 1 \times 10^{4}, 5 \times 10^{4}$ and $1 \times 10^{5}$ cells $\mathrm{mL}^{-1}$ for the 
seeding density experiment and $2 \times 10^{4}$ cells $\mathrm{mL}^{-1}$ for subsequent proliferation assays with $C$. fritschii extracts.

For seeding density experiments, cell index was measured every $1 \mathrm{~min}$ for $2 \mathrm{~h}$ for attachment of cells followed by readings every $15 \mathrm{~min}$ for $48 \mathrm{~h}$ for proliferation.

For C. fritschii extract proliferation activity, cell index was measured every 1 min for $2 \mathrm{~h}$ for attachment of cells followed by readings every $5 \mathrm{~min}$ for $22 \mathrm{~h}$ for pre-treatment proliferation. After $24 \mathrm{~h}, 50 \mu \mathrm{L}$ of the pooled control extracts at 10, 5, 1, 0.5, 0.1 and $0.05 \mu \mathrm{g} \mathrm{mL}^{-1}(\mathrm{n}=2)$ were then added and measured every $1 \mathrm{~min}$ for $1 \mathrm{~h}$ to evaluate initial effect of extract followed by readings every $5 \mathrm{~min}$ for a further $24 \mathrm{~h}$ for post treatment proliferation. A blank (DMSO) was also used to see the effects of the vehicle control on proliferation.

The assay above was repeated with control and UV-B pooled extracts at concentrations of $0.5,0.25$ and $0.1 \mu \mathrm{g} \mathrm{mL}^{-1}(\mathrm{n}=2)$ and proliferation was measured over $72 \mathrm{~h}$.

\subsubsection{Antibacterial activity of extracts}

Antibacterial activity of extracts were assessed against the test bacterium Staphylococcus epidermis, ATCC ${ }^{\circledR} 12228^{\mathrm{TM}}$ (S. epidermis) using the microdilution method. A stock of S. epidermis was inoculated into $50 \mathrm{~mL}$ (TSB) and incubated overnight at $35^{\circ} \mathrm{C}$ with shaking at $120 \mathrm{rpm}$ for growth of an even cell suspension. The inoculum was centrifuged (3500 rcf, $10 \mathrm{~min}$ ), re-suspended and diluted to an OD of 0.1 using $10 \%$ TSB in $\mathrm{H}_{2} \mathrm{O}$ for use in the assay.

Pooled UV-B and control extracts (Section 1.8.1.3 and 1.8.1.4) were diluted with culture broth (10\% TSB) to a concentration of $0.5 \mathrm{mg} \mathrm{mL}^{-1}(1: 20)$. Further 1:2 serial dilutions were performed by addition of culture broth to produce concentrations ranging from $0.5-0.0156 \mathrm{mg} \mathrm{mL}^{-1} .270 \mu \mathrm{L}$ of each concentration was added to wells of a 96 microplate along with $30 \mu \mathrm{L}$ of bacterial suspension $(n=2)$. Growth controls were used with DMSO diluted as above and $30 \mu \mathrm{L}$ of bacterial suspension. Wells were included with diluted extracts only (no bacterial cells) to account for pigment interference. The plates were incubated for $24 \mathrm{~h}$ at $35{ }^{\circ} \mathrm{C}$ and bacterial growth was measured by an end-point growth reading measured at $590 \mathrm{~nm}$.

Antibacterial activity was repeated with individual UV-B extracts and control extracts at concentrations of $0.5-0.03125 \mathrm{mg} \mathrm{mL}^{-1}$. 


\section{Chapter 2: Time-Series Metabolite Level Investigation of Chlorogloeopsis fritschii, PCC 6912, During Standard Growth Conditions}

Aims

- Investigations into the metabolite profiles of $C$. fritschii during standard conditions.

- To optimise the extraction of metabolites from C. fritschii for GC-MS analysis.

- To evaluate the similarities and difference in growth, protein, carbohydrate content and GC-MS profiles of axenic and xenic C. fritschii cultures over 28 days.

- To identify metabolites produced by $C$. fritschii during 120 days of growth.

- Evaluation of metabolite level changes over $48 \mathrm{~h}$ to use as a control for UV exposure experiments (Chapter 3). 


\section{Introduction}

Use of metabolomics in cyanobacterial research is a useful tool. As candidates for industrial biotechnology it is important to evaluate the production of metabolites to help understand the mechanisms behind their growth and survival. This can also give an insight into secondary metabolite production. GC-MS is a well-used technique in metabolomics research giving advantages over other analytical techniques. It is a wellknown metabolomics tool to investigate metabolite levels in a variety of biological samples. The study of plants and microalgae under different growth and development phases [91-95], and during abiotic stress response [96-98] have been widely investigated. Little work has been conducted on metabolite profiles within cyanobacteria with the majority of research on metabolite changes during abiotic stress including; $\mathrm{CO}_{2}$ limitation [53] and UV-B/UV-A exposure [52,99].

Extraction of a wide variety of metabolites is well documented in metabolomics research and is dependent on the material to be extracted and the intended metabolites to be evaluated. Extraction methods for GC-MS analysis in a variety of biological material is well documented, from human tissue, plants, and microorganisms including; the yeast S. cerevisiae [100], the bacterium Escherichia coli [101] and the diatom Skeletonema marinoi [94]. A variety of metabolite classes need to be extracted to gain a good insight into the metabolome.

It is important to analyse the metabolome during standard conditions as well as abiotic stress to get a better understanding of metabolism within cyanobacteria. This can then be used to identify potentially relevant metabolites for use in industrial biotechnology. To begin, an assessment of extraction techniques of metabolites from $C$. fritschii was conducted to determine the best combination of solvent and mechanical disruption to extract a variety of metabolites from $C$. fritschii for GC-MS analysis. All subsequent experiments used the same techniques and analysis for consistency throughout the work. The analysis of axenic and xenic cultures investigates any differences in metabolites identified between the two culture conditions. A long term, 120 day timeseries experiment was then investigated to analyse the metabolite level changes over different growth phases. A $48 \mathrm{~h}$ control experiment was undertaken to analyse the changes in metabolite levels during standard conditions over a short time-series. These results were then used to compare to UV-A and UV-B exposure experiments (Chapter 
3). These experiments can be used to improve knowledge-base in novel non-model cyanobacterial strains such as $C$. fritschii. 


\subsection{Experimental design}

\subsubsection{Evaluation of intracellular metabolite extraction techniques}

See Section 1.3.1 for C. fritschii growth, harvest and extraction of intracellular metabolites and Section 1.3.3-1.3.6 for GC-MS derivatisation and analysis.

\subsubsection{Axenic vs. Xenic: 28 day analysis of $C$. fritschii}

C. fritschii cultures were pre-grown for 6 days prior to experimental analysis (Section 1.1). After 6 days of pre-growth, triplicate experimental axenic and xenic (non-axenic) C. fritschii cultures $\left(\mathrm{OD}_{750 \mathrm{~nm}}\right.$ of approx. 0.1 and 0.13 respectively) were grown at a temperature of $27^{\circ} \mathrm{C}$ under continuous PAR $\left(15 \mu \mathrm{mol} \mathrm{m}^{-1} \mathrm{~s}^{-1}\right)$ and shaking at $100 \mathrm{rpm}$. For time course analysis samples were harvested at $0,4,8,14,20$ and $28 \mathrm{~d}$ for dry weight (Section 1.2), total protein (Section 1.6), total carbohydrate (Section 1.7) and GC-MS analysis (Sections 1.3.2 - 1.3.6). Significant differeces in metabolite levels were calculated between time points (d); 0 vs. 4,4 vs. 8,8 vs. 14,14 vs. 20 and 20 vs. 28.

\subsubsection{0 day analysis of C. fritschii}

C. fritschii cultures were pre-grown for 6 days prior to experimental analysis (Section 1.1). After 6 days of pre-growth, triplicate experimental $C$. fritschii cultures (OD ${ }_{750 \mathrm{~nm}}$ of approx. 0.08) were grown at a temperature of $27{ }^{\circ} \mathrm{C}$ under continuous PAR (15 $\mu \mathrm{mol} \mathrm{m} \mathrm{m}^{-1} \mathrm{~s}^{-1}$ ) and shaking at $100 \mathrm{rpm}$. For time course analysis samples were harvested every 8 days for 120 days (0-120) for dry weight (Section 1.2) and GC-MS analysis (Sections 1.3.2 - 1.3.6). Significant differeces in metabolite levels were calculated between time points (d); 0 vs. 8,8 vs. 16,16 vs. 24,24 vs. 32,32 vs. 40,40 vs. 48,48 vs. 56,56 vs. 64,64 vs. 72,72 vs. 80,80 vs. 88,88 vs. 96, 96 vs. 104,104 vs. 112 and 112 vs. 120 unless otherwise stated.

\subsection{4 $48 \mathrm{~h}$ analysis of $C$. fritschii}

C. fritschii cultures were pre-grown for 6 days prior to experimental analysis (Section 1.1). After 6 days of pre-growth, triplicate experimental $C$. fritschii cultures $\left(\mathrm{OD}_{750 \mathrm{~nm}}\right.$ of approx. 0.13) were grown at a temperature of $27{ }^{\circ} \mathrm{C}$ under continuous PAR (15 $\mu \mathrm{mol} \mathrm{m} \mathrm{s}^{-1}$ ) and shaking at $100 \mathrm{rpm}$. For time course analysis samples were harvested at $0,2,6,12,24$ and $48 \mathrm{~h}$ for dry weight (Section 1.2) and GC-MS analysis (Sections

\subsection{2-1.3.6).}




\subsection{Results}

\subsubsection{Evaluation of intracellular metabolite extraction techniques}

Different solvents combined with disruption techniques were evaluated to determine the best extraction protocol of metabolites from $C$. fritschii. The number of peaks detected, the number of identifiable peaks and the recovery of different compounds of different polarity of each protocol was evaluated [94].

Table 15: Combination of disruption techniques and solvents for extraction of metabolites from $C$. fritschii

\begin{tabular}{ll}
\hline Disruption technique & Solvent \\
\hline (A) Ice-bath & (1) $\mathrm{MeOH}$ \\
& (2) $\mathrm{CHCl}_{3}$ \\
& (3) $\mathrm{CHCl}_{3}: \mathrm{MeOH}$ \\
\hline (B) Sonication & (1) $\mathrm{MeOH}$ \\
& (2) $\mathrm{CHCl}_{3}$ \\
& (3) $\mathrm{CHCl}_{3}: \mathrm{MeOH}$ \\
\hline (C) Freeze-thaw & (1) $\mathrm{MeOH}$ \\
& (2) $\mathrm{CHCl}_{3}$ \\
& (3) $\mathrm{CHCl}_{3}: \mathrm{MeOH}$ \\
\hline
\end{tabular}

To assess the extraction of metabolites, $C$. fritschii at an OD of 1 was extracted using different mechanical disruption techniques (ice-bath, sonication, freeze-thaw) combined with the extraction solvents $\mathrm{MeOH}, \mathrm{CHCl}_{3}$ and $\mathrm{CHCl}_{3}: \mathrm{MeOH}$ (Table 15). To visualise the differences between extraction procedures, the aligned IS matrix from SpectConnect was analysed using PCA (Figure 8) in MetaboAnalyst. This plot condenses the data into three principle components and shows the total variation between samples as 53.9\%. A clear separation was seen between $\mathrm{CHCl}_{3}$ and the other solvent types with $40.8 \%$ of the variation explained by PC1. Less variation was seen between $\mathrm{MeOH}$ and $\mathrm{CHCl}_{3}: \mathrm{MeOH}$ indicating these extraction solvents are more closely related. Strong clustering was observed within each solvent. No clustering was observed between disruption techniques. 


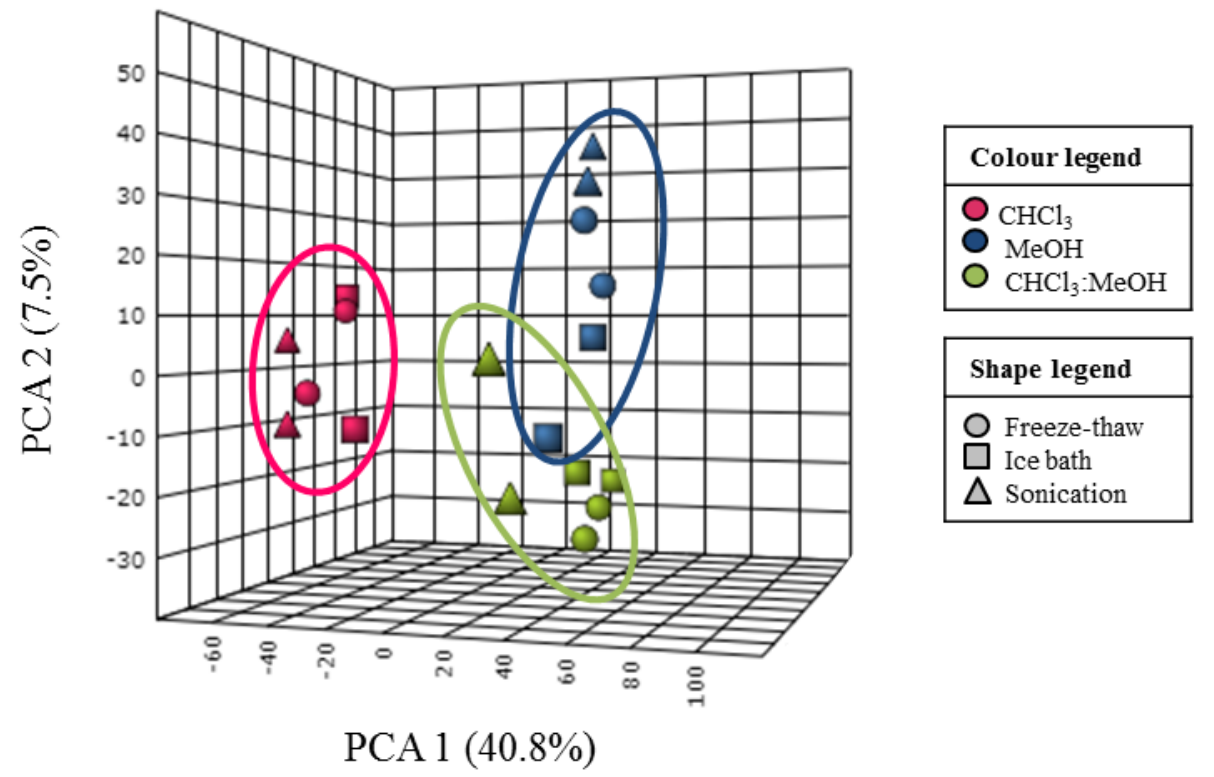

Figure 8: Principle component analysis (PCA) of metabolites extracted using different techniques and solvents. Plot generated from 288 peaks detected across all samples. Each ring represents the distribution of the three different solvents used.

The results of the PCA were confirmed by analysis using ANOVA- Simultaneous Component Analysis (ASCA). 54.92\% of the variation between samples can be explained by the technique in component 1 with sonication samples showing more variation than freeze thaw and ice bath (Figure 9a). 87.1\% of the variation can be explained by the solvent used (Figure 9b) with $\mathrm{CHCl}_{3}$ less similar to $\mathrm{MeOH}$ and $\mathrm{MeOH}: \mathrm{CHCl}_{3}$ extraction as seen in the PCA (Figure 8).
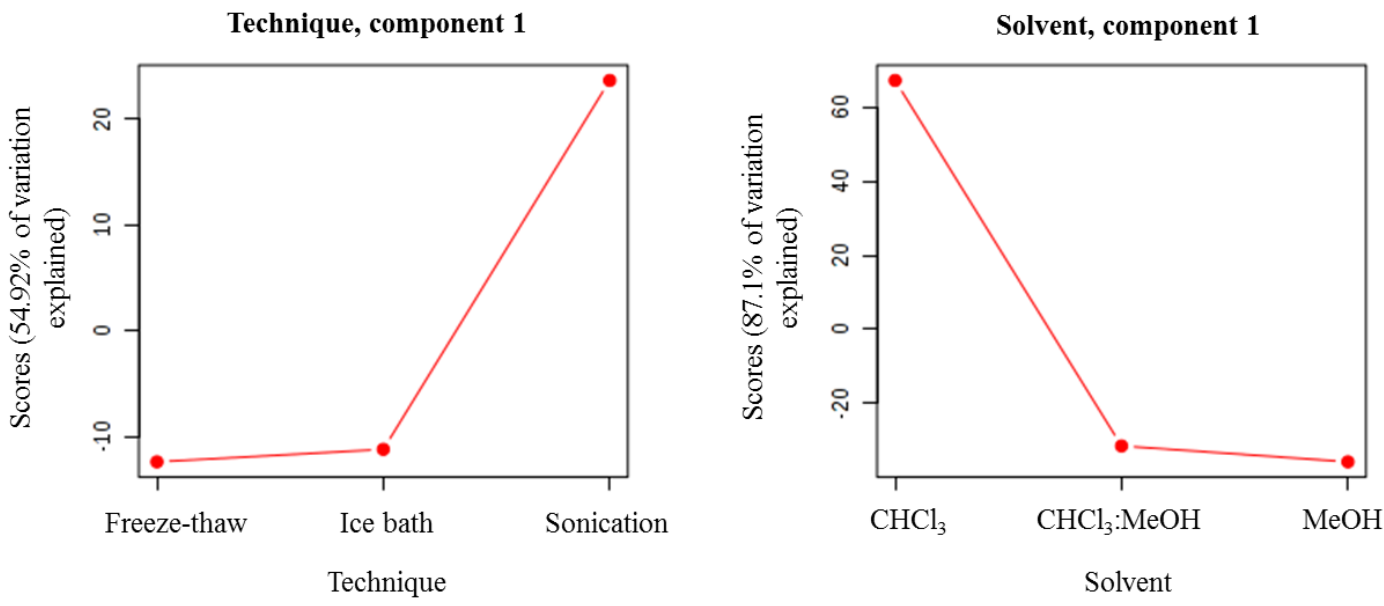

Figure 9: ANOVA-Simultaneous Component Analysis (ASCA) scree plots showing major patterns associated with A) technique and B) solvent generated from total peaks detected across all samples. 
The hierarchial heatmap (Figure 10) again shows a high statistical significance $(p<0.05)$ between solvents (57 peaks), with less significance observed between technique (21).

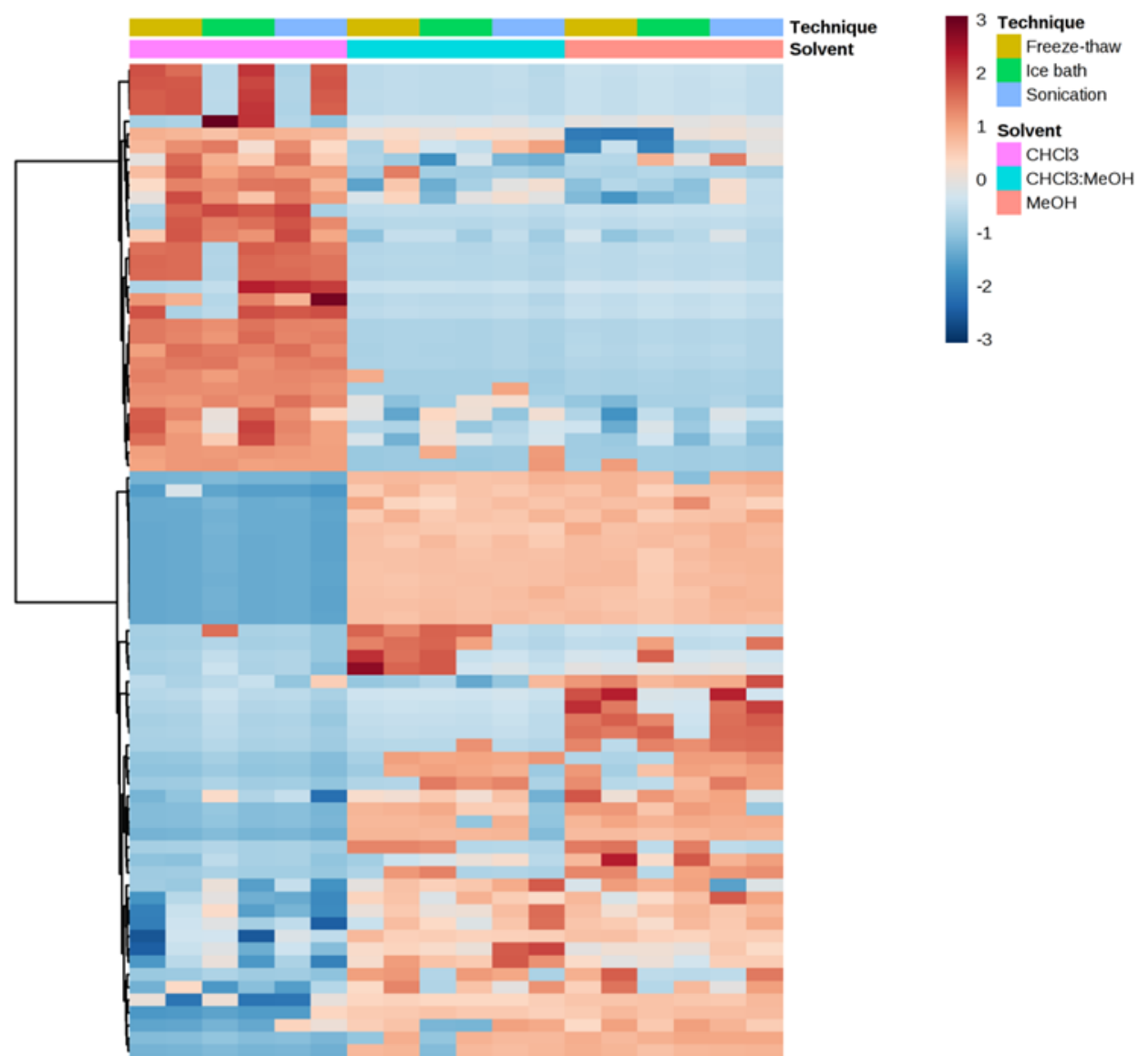

Figure 10: Hierarchial heatmap visualisation of the significanty different peaks $(p<0.05)$, using identified peaks only, comparing each extraction protocol using a two-way between samples ANOVA in MetaboAnalyst. Clustering distance was measured using Euclidean and the clustering algorithm was ward. The samples are ordered by solvent; $\mathrm{CHCl}_{3}, \mathrm{CHCl}_{3}: \mathrm{MeOH}$ and $\mathrm{MeOH}$ from left to right respectively.

Overall a total of 288 peaks were detected across the whole data series. Using the Golm Metabolome Database (GMD) and NIST library, a total of 158 peaks were identified (Level 2 [63]) with a match factor $\geq 60 \%$ with 130 remaining unassigned (Level 4 [63]). The identified compounds were classified according to their identification using GMD/NIST library as; acids, alcohols, hydrocarbons, amino acids/alcohols, aromatics, esters, ethers and ketones, fatty acids and fatty alcohols, heterocycles, N-compounds, other, sterols and sugars (and derivatives). 
Peak numbers across $\mathrm{MeOH}$ and $\mathrm{CHCl}_{3}: \mathrm{MeOH}$ for all disruption techniques were similar, with peak identification of all $\mathrm{CHCl}_{3}: \mathrm{MeOH}$ protocols slightly higher overall. All $\mathrm{CHCl}_{3}$ extractions had the lowest peak detection and number of identifications. Sonication had the highest number of detected peaks with each solvent system (Figure 11).

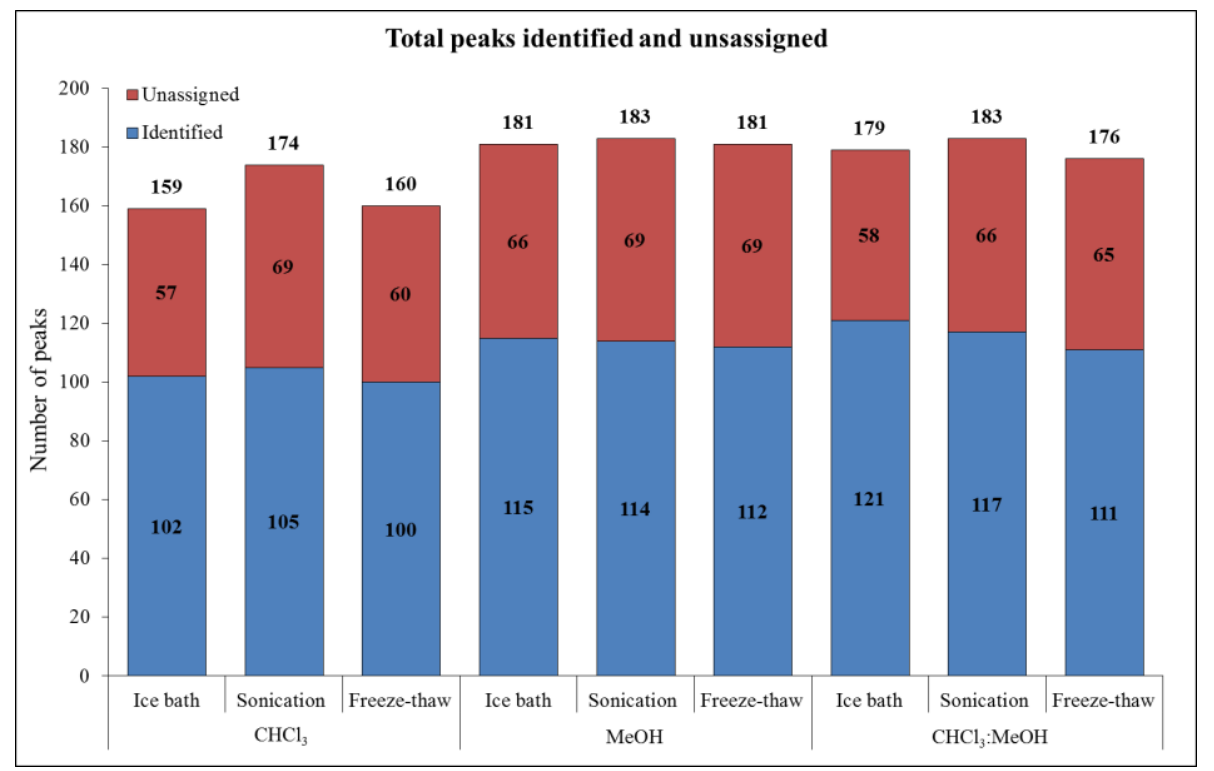

Figure 11: Total number of peaks detected across all extraction protocols including number of identified and unassigned peaks.

The different procedures extracted compounds of varying chemical classes. The nonpolar solvent $\mathrm{CHCl}_{3}$ extracted more non-polar compounds such as fatty acids and sterols. The polar protic solvent $\mathrm{MeOH}$ extracted a higher percentage of polar compound classes such as sugars and amino acids (Figure 12). 


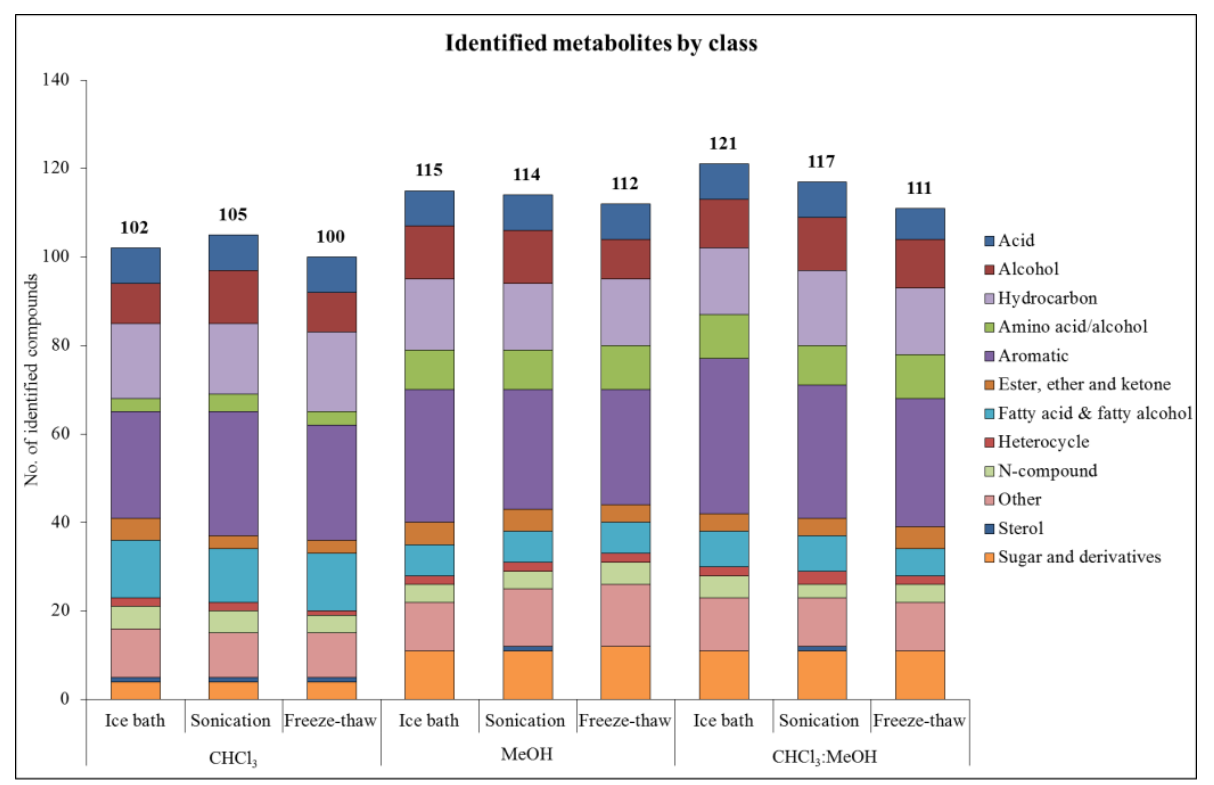

Figure 12: Number of identified peaks extracted from $C$. fritschii cells by chemical class for each extraction protocol.

49 metabolites were identified with biological relevance to metabolic pathways within cyanobacteria including fatty acid and amino acid biosynthesis, galactose, fructose and mannose metabolism, glycerophospholipid metabolism to name a few. Four metabolites involved in fatty acid biosynthesis were extracted in all extraction protocols. Sugars involved in fructose, mannose, galactose and sucrose metabolism were extracted in $\mathrm{MeOH}$ and $\mathrm{CHCl}_{3}: \mathrm{MeOH}$ protocols. Whereas sugar alcohols involved in fructose and mannose metabolism were extracted in all solvents. Acyl glycerols involved in glycerolipid biosynthesis were extracted in $\mathrm{CHCl}_{3}$ protocols only.

From these results, a biphasic liquid extraction protocol using $\mathrm{CHCl}_{3}: \mathrm{MeOH}$ coupled with sonication was chosen for subsequent experiments. A sonicator bath was replaced with a sonicator probe to ensure maximum cell disruption and extraction of metabolites. $\mathrm{H}_{2} \mathrm{O}$ added as a phase separator to enhance separation of polar and nonpolar phases.

\subsubsection{Axenic vs. xenic: Metabolite profiling of $C$. fritschii over 28 days}

Both axenic and xenic $C$. fritschii cultures were compared to determine any differences between the two culture conditions. In this work an axenic culture is defined as the cultivation of $C$. fritschii only (no contamination with other microorganisms) whereas a xenic culture refers to $C$. fritschii cultures grown with a small proportion of other 
microorganisms such as other bacteria (as seen by streaking samples on nutrient agar). The analyses of metabolite levels of both axenic and xenic cultures were examined to see any major differences over 28 days of cultivation.

The starting biomass concentration $\left(\mathrm{mg} \mathrm{L}^{-1}\right)$ was lower for axenic compared to xenic cultures due to its slower growth. Similar growth rates were measured overall between days 8 and 28 (axenic, $\mu=0.233 \pm 0.009$; xenic, $0.234 \pm 0.012$, Figure 13). Differences in growth rate can be seen up to day 8 (Figure 14). A lag phase was observed with xenic cultures up to day 4 with a growth rate of $\mu=0.55 \pm 0.012$ compared to the axenic cultures, $\mu=0.88 \pm 0.035$ ( 0 vs. $4 \mathrm{~d}, p<0.01)$ this was followed by significant increase between days 4 and 8 in the xenic cultures $(\mu=1.06 \pm 0.098, p<0.01)$ compared to axenic cultures $(\mu=0.78 \pm 0.089, p>0.05)$.

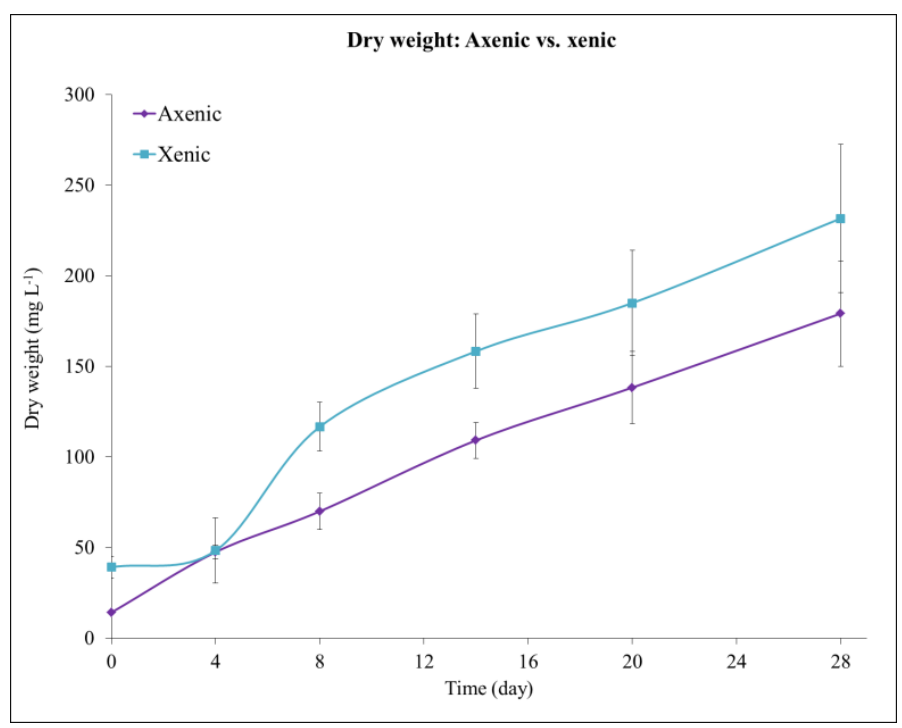

Figure 13: Dry weight of axenic and xenic $C$. fritschii cultures $(\mathrm{n}=3)$ during growth over 28 days under control conditions sampling at $0,4,8,14,20$ and 28 days. Each data point is the mean value of biological triplicates \pm standard error $(\mathrm{SE})$. 


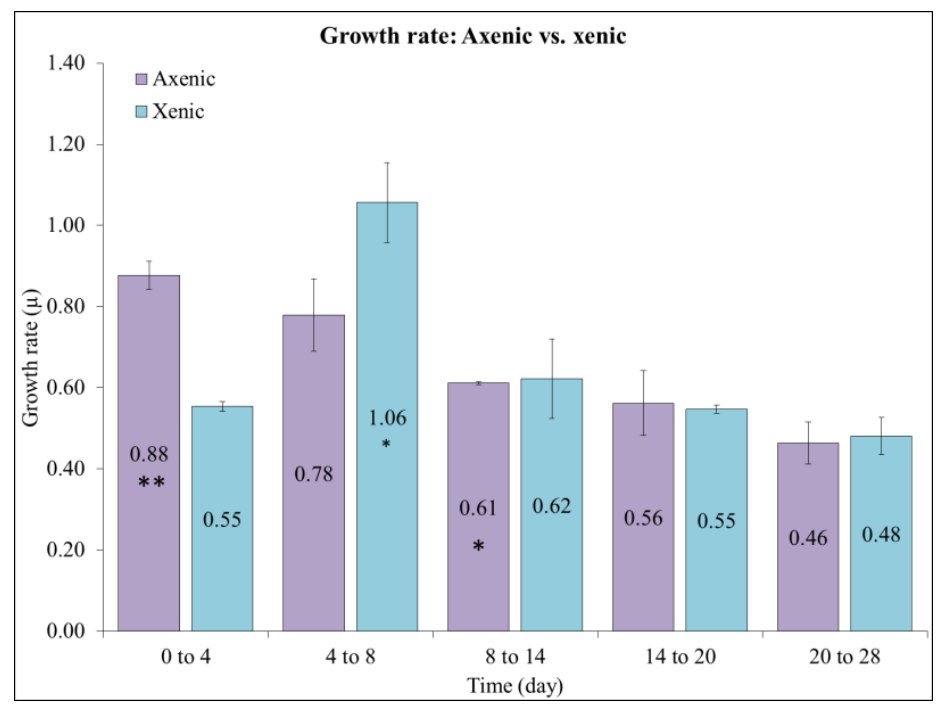

Figure 14: Specific growth rate $(\mu)$ of axenic and xenic $C$. fritschii cultures between time points showing mean values \pm SE. Significant difference was measured using a two-sample t-Test with equal variance comparing each time-point, $*=0.05>p \geq 0.01, * *=0.01>p \geq 0.001$ and $* * *=p<0.001$.

Bulk carbohydrate and protein content was measured using the colourimetric sulfuric acid-phenol [89] and Lowry [87] method respectively. Results showed significant differences in carbohydrate content between days 4 and 8 in axenic cultures $(p<0.05)$ with no significant difference in xenic cultures (Figure 15A). Protein levels remained steady throughout the time series with no significance in both axenic and xenic cultures $(p>0.05)$ (Figure 15B). Comparing the two cultures, a statistical significance was observed at day $0(p<0.05)$ and day $8(p<0.001)$ for carbohydrate content but no significance was observed comparing protein content (axenic vs. xenic, $p>0.05$ ). 


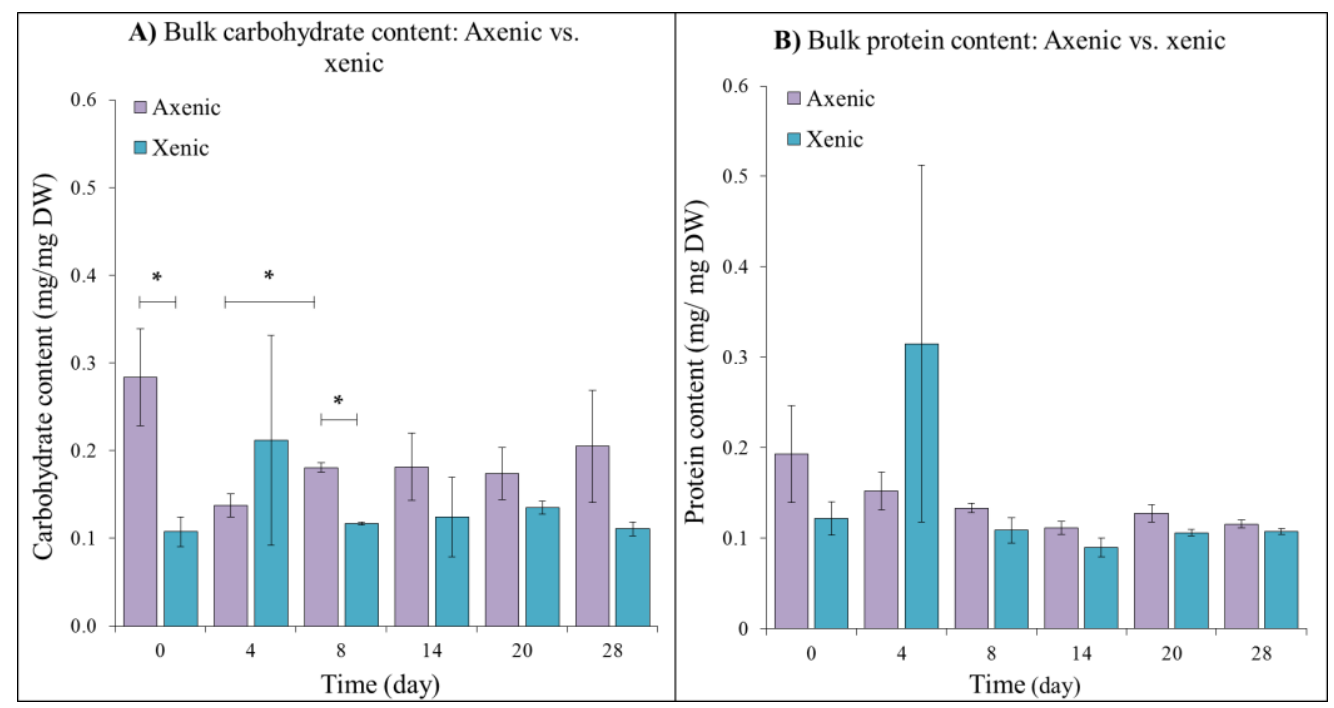

Figure 15: (A) Bulk carbohydrate content of axenic and xenic $C$. fritschii cultures during 28 days of growth. (B) Bulk protein content of axenic and xenic C. fritschii cultures during 28 days of growth. Significant difference was measured using a two-sample t-Test with equal variance comparing each time-point and between treatments, $*=0.05>p \geq 0.01,{ }^{* *}=0.01>p \geq 0.001$ and ${ }^{* * *}=p<0.001$.

\subsubsection{Intracellular metabolites}

564 and 535 peaks were detected across the whole 28 day time-series in axenic and xenic cultures respectively. From axenic cultures 241 peaks were identified with a match factor $\geq 60 \%$ (level 2 [63]) with 323 remaining unassigned (level 4 [63]) whereas 238 peaks from xenic cultures were identified with a match factor $\geq 60 \%$ (level 2 ) with 297 peaks left unassigned (level 4).

In axenic cultures, the greatest significant difference in metabolite levels (two-sample t-Test, $p<0.05$ ) was observed between 0 and 4 days with $93 \%$ of peaks decreasing in abundance (433 with negative $\log 2$ Fold Change (FC)). Significant difference in metabolite levels between time points decreased with increasing time. The lowest significant differences was calculated between 20 and 28 days with 93 peaks reducing in levels and 42 increasing in levels between the two time points (Table 16).

In xenic cultures the greatest significant difference in metabolite levels was seen between days 0 and 4 with $95 \%$ of peaks decreasing in abundance (negative $\log 2 \mathrm{FC}$ ). The lowest significant difference was calculated comparing days 14 and 20 with the majority of peaks increasing in abundance (66\%, Table 17). 
Table 16: Comparison of significantly different $(p<0.05)$ peaks (out of 564) and number of those significantly different peaks with a positive or negative log2 Fold Change (FC) within axenic cultures. Both significant different and $\log 2 \mathrm{FC}$ was calculated comparing between time points (d); 0 vs. 4,4 vs. 8,8 vs. 14,14 vs. 20 and 20 vs. 28 .

\begin{tabular}{cccc}
\hline $\begin{array}{c}\text { Axenic } \\
\text { (d) }\end{array}$ & $\begin{array}{c}\text { No. of peaks, } \\
\boldsymbol{p}^{<0.0 \mathbf{0 .}^{\mathbf{a}}}\end{array}$ & $\begin{array}{c}\text { No. of peaks, } \\
\text { positive log2FC }\end{array}$ & $\begin{array}{c}\text { No. of peaks, } \\
\text { negative log2FC }\end{array}$ \\
\hline $\mathbf{0}$ vs. $\mathbf{4}$ & 466 & 33 & 433 \\
$\mathbf{4}$ vs. 8 & 407 & 58 & 349 \\
$\mathbf{8}$ vs. $\mathbf{1 4}$ & 183 & 113 & 70 \\
$\mathbf{1 4}$ vs. $\mathbf{2 0}$ & 141 & 60 & 81 \\
$\mathbf{2 0}$ vs. $\mathbf{2 8}$ & 135 & 42 & 93 \\
\hline
\end{tabular}

${ }^{\mathrm{a}}=$ Significant difference was calculated using a two sample t-Test with equal variance.

Table 17: Comparison of significantly different $(p<0.05)$ peaks (out of 535) and number of those significantly different peaks with a positive or negative $\log 2 \mathrm{FC}$ within xenic cultures. Both significance and $\log 2 \mathrm{FC}$ was calculated comparing between time points (d); 0 vs. 4,4 vs. 8,8 vs. 14,14 vs. 20 and 20 vs. 28.

\begin{tabular}{cccc}
\hline $\begin{array}{c}\text { Xenic } \\
\text { (d) }\end{array}$ & $\begin{array}{c}\text { No. of peaks, } \\
\boldsymbol{p}<\mathbf{0 . 0 5}^{\mathbf{a}}\end{array}$ & $\begin{array}{c}\text { No. of peaks, } \\
\text { positive log2FC }\end{array}$ & $\begin{array}{c}\text { No. of peaks, } \\
\text { negative log2FC }\end{array}$ \\
\hline $\mathbf{0}$ vs. $\mathbf{4}$ & 181 & 10 & 171 \\
$\mathbf{4}$ vs. 8 & 177 & 163 & 14 \\
$\mathbf{8}$ vs. $\mathbf{1 4}$ & 170 & 50 & 120 \\
$\mathbf{1 4}$ vs. $\mathbf{2 0}$ & 131 & 86 & 45 \\
$\mathbf{2 0}$ vs. $\mathbf{2 8}$ & 151 & 37 & 114 \\
\hline
\end{tabular}

${ }^{\mathrm{a}}=$ Significant difference was calculated using a two sample t-Test with equal variance. 
The peak lists were further analysed using PCA. The normalised abundance data (by dry weight and internal standard) was further processed in MetaboAnalyst by normalising by the sum followed by log transformation and Pareto scaling. Total variance was calculated as 38.8\% (PC1 17\%, PC2 11.8\% and PC3 10\%) for axenic cultures. Clustering of peaks was observed between day 0,4 and 8 with separate clustering of day 14 and 20 across PC1. Day 28 showed most separation compared to each time point (Figure 16A). Total variance for xenic cultures was calculated as 42.8\% (PC1 16.1\%, PC2 $15.6 \%$ and PC3 11.1\%). Clustering of peaks by replicates was observed with good separation of time points (Figure 16B).
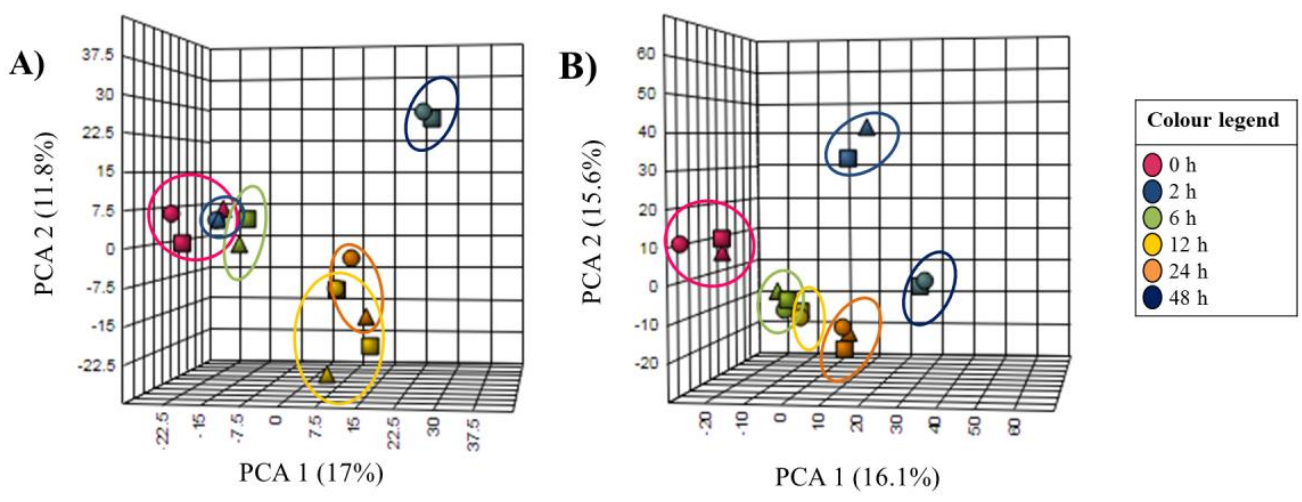

Figure 16: PCA of detected peaks over 28 days from A) axenic and B) xenic $C$. fritschii cultures. Each ring represents distribution of replicates from each time points. Samples from axenic day 4 (S2), 8 (S1) and 28 (S3) and xenic day 4 (S1), 14 (S3) and 28 (S3) have been omitted. Data was normalised by sum, $\log$ transformed with Pareto scaling.

Peak matrices for both axenic and xenic cultures were analysed for significant differences using a one-way ANOVA. 100 peaks were found to be significantly different $(p<0.05)$ across the whole time-series in axenic cultures with 22 remaining significant after Bonferroni (BF) correction. This correction was chosen to adjust the $p$ values (by multiplying by number of tests, $k$, in this case 137 ) thus reducing the amount of false-positives due to the multiple analysis of a single data set [44,102]. 135 peaks with significant difference $(p<0.05)$ across the whole time-series were found in xenic cultures, 14 of those remained significant after BF correction.

A total of 70 metabolites were identified within the two data sets with biological relevance to cyanobacterial metabolism $73 \%$ (51) of those were identified in both axenic and xenic cultures. 27\% (19) were different between the two with 11 found exclusively in axenic cultures (Table S1) and 8 from xenic cultures only (Table S2). 
The greatest significant difference in metabolite levels was seen within the first 4 days between axenic and xenic cultures with 34 significantly different metabolites (axenic vs. xenic, 0 vs. 4 d) this then decreased with increasing time to a minimum of 7 significantly different metabolites comparing days 14 and 20 (axenic vs. xenic, Table 18). Overall axenic cultures showed more significant differences between time points compared to xenic cultures, but little differences were observed after 4 days comparing axenic and xenic results (Table 18).

Table 18: Number of significantly different $(p<0.05)$ biologically relevant metabolite levels from axenic and xenic cultures including comparison between the two. Significant difference was calculated between axenic and xenic by comparing $\log 2 \mathrm{FC}$ between the time points (d); 0 vs. 4,4 vs. 8,8 vs. 14 , 14 vs. 20 and 20 vs. 28.

\begin{tabular}{cccc}
\hline Days (d) & $\begin{array}{c}\text { No. of axenic } \\
\text { peaks, } \boldsymbol{p}<\mathbf{0 . 0 5}^{\mathrm{a}}\end{array}$ & $\begin{array}{c}\text { No. of xenic peaks, } \\
\boldsymbol{p}<\mathbf{0 . 0 5}^{\mathrm{a}}\end{array}$ & $\begin{array}{c}\text { No. of peaks } \\
\boldsymbol{p}<\mathbf{0 . 0 5}^{\mathbf{a}} \text {, axenic vs. } \\
\text { xenic }\end{array}$ \\
\hline 0 vs. $\mathbf{4}$ & 46 & 20 & 34 \\
$\mathbf{4}$ vs. 8 & 34 & 28 & 11 \\
$\mathbf{8}$ vs. $\mathbf{1 4}$ & 14 & 21 & 8 \\
$\mathbf{1 4}$ vs. $\mathbf{2 0}$ & 12 & 9 & 7 \\
$\mathbf{2 0}$ vs. 28 & 15 & 19 & 11 \\
\hline
\end{tabular}

${ }^{\mathrm{a}}=$ Significant difference was calculated using a two sample t-Test with equal variance.

The $\%$ composition of all detected metabolites over the 28 days was very similar between the two cultures (Figure 17A and Figure 18A).

Axenic and xenic cultures detected 13 and 12 amino acids respectively across the whole time-series with varying abundances and significance. These included the hydrophobic aliphatic; alanine (ala), isoleucine (ile), and valine (val), the hydrophic aromatic; phenylalanine (phe) and tyrosine (tyr), the polar neutral; serine (ser) and threonine (thr), the acidic-polar; aspartate (asp) and glutamate (glu) the basic-polar; arginine (arg) and lysine (lys) as well as glycine (gly) and proline (pro).

Six (6, axenic and xenic) metabolites were found to be involved in fatty acid biosynthesis with 9 (axenic) and 7 (xenic) involved in sugar metabolism with 3 identified metabolites involved in the tricarboxylic acid (TCA) cycle in both cultures. 
Differences in axenic cultures included the amino acid ile and tyr as well as the sugars mannose, fructose and galactose (Figure 17). Difference in xenic cultures included ala and glucose to name a few (Figure 18).

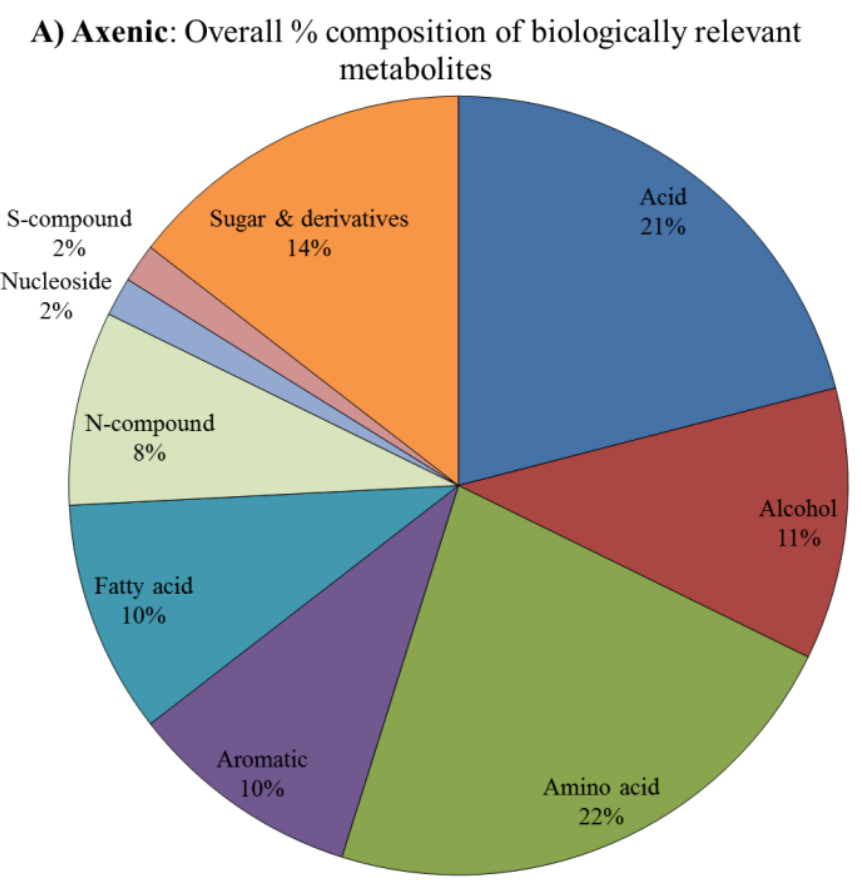

\begin{tabular}{|c|c|c|c|}
\hline B) Acids (TCA) & C) Amino acids & D) Fatty acids & E) Sugars \& derivatives \\
\hline $\begin{array}{ll}\text { - } & \text { Fumarate } \\
\text { - } & \text { Malate } \\
\text { - } & \text { Succinate }\end{array}$ & $\begin{array}{ll}\text { - } & \text { Isoleucine, Valine } \\
\text { - } & \text { Phenylalanine, } \\
\text { Tyrosine } \\
\text { - } & \text { Serine, Threonine } \\
\text { - } & \text { Aspartate, Glutamate } \\
\text { - } & \text { Arginine, Lysine } \\
\text { - } & \text { Glycine, Proline } \\
\text { - } & \text { Ornithine }\end{array}$ & $\begin{array}{ll}\text { - } & \text { Capric acid } \\
\text { - } & \text { Lauric acid } \\
\text { - } & \text { Mystiric acid } \\
\text { - } & \text { Palmitic acid } \\
\text { - } & \text { Stearic acid } \\
\text { - } & \text { Eicosanoic acid }\end{array}$ & $\begin{array}{l}\text { - Rhamnose } \\
\text { - Mannose } \\
\text { - Fructose } \\
\text { - Galactose } \\
\text { - Raffinose } \\
\text { - Sucrose } \\
\text { - Lyxose } \\
\text { - Trehalose } \\
\text { - Xylulose-5-phosphate }\end{array}$ \\
\hline
\end{tabular}

Figure 17: Overview of biologically relevant metabolites detected, at each time-point combined, in axenic cultures over the whole time-series of 28 days. A) Overall \% composition of detected metabolites by chemical class. B, C, D and E) Identified metabolites by class as acids (involved in TCA), amino acids, fatty acids and sugars (and derivatives) over the whole time-series. Metabolites in bold were identified in axenic experiment only. 
A) Xenic: Overall \% composition of biologically relevant metabolites
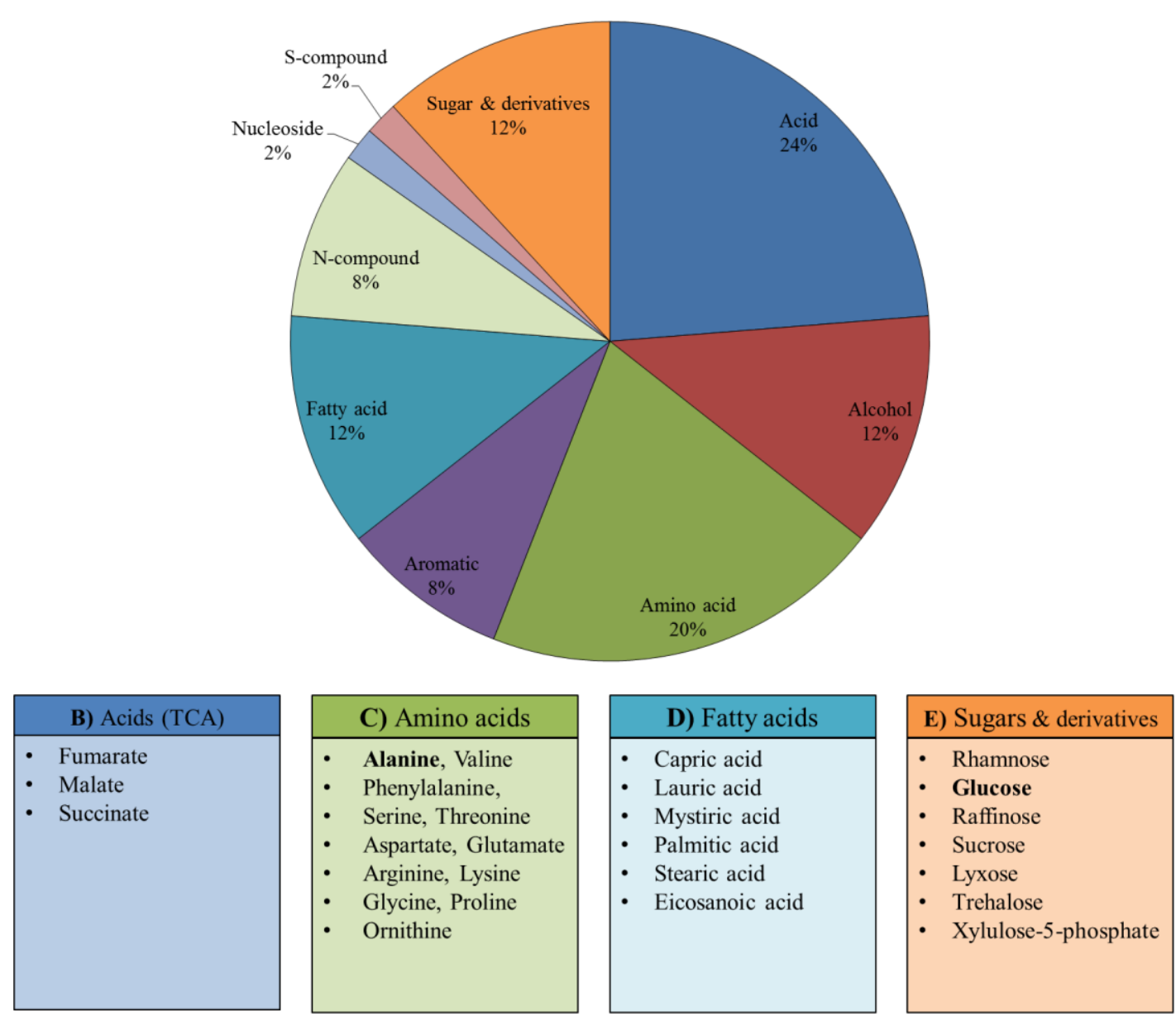

Figure 18: Overview of biologically relevant metabolites detected at each time-point combined, in xenic cultures over the whole time-series of 28 days. A) Overall \% composition of detected metabolites by chemical class. B, C, D and E) Identified metabolites by class as acids (involved in TCA), amino acids, fatty acids and sugars (and derivatives) over the whole time-series. Metabolites in bold were identified in xenic experiment only. 


\subsubsection{Metabolite profile of $C$. fritschii over 120 days}

A long term experiment was conducted over a 120 day time-series to analyse the changes in metabolite levels during the growth of $C$. fritschii. Samples were harvested every 8 days for 120 days from three separate flasks (biological replicates, $n=3$ ) and metabolite profiles analysed. As expected, significant differences in dry weight comparing day 0 and each time point was observed (day 0 vs. 16 up to $120, p<0.05$, Figure 19). Less significant difference in dry weight values was seen between time points and was only observed comparing day 8 vs.16 ( $p<0.05), 16$ vs. $24(p<0.01)$ and 24 vs. $40(p<0.001)$.

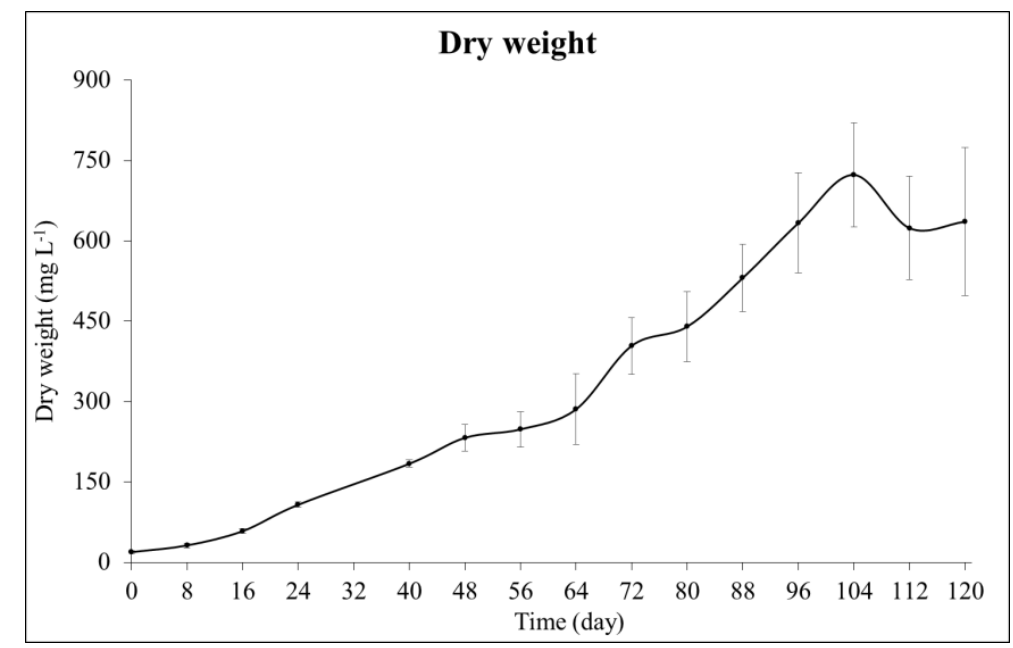

Figure 19: Dry weight of $C$. fritschii cultures $(\mathrm{n}=3)$ during growth over 120 days under control conditions sampling every 8 days between 0 and 120 days, day 32 data omitted. Each data point is the mean value of biological triplicates $\pm \mathrm{SE}$.

Triplicate flasks had similar dry weight values up to day 40. After this, an increased SE was calculated between the replicates (Figure 20). All GC-MS data was normalised to dry weight to counteract variations in biomass between each sample. 


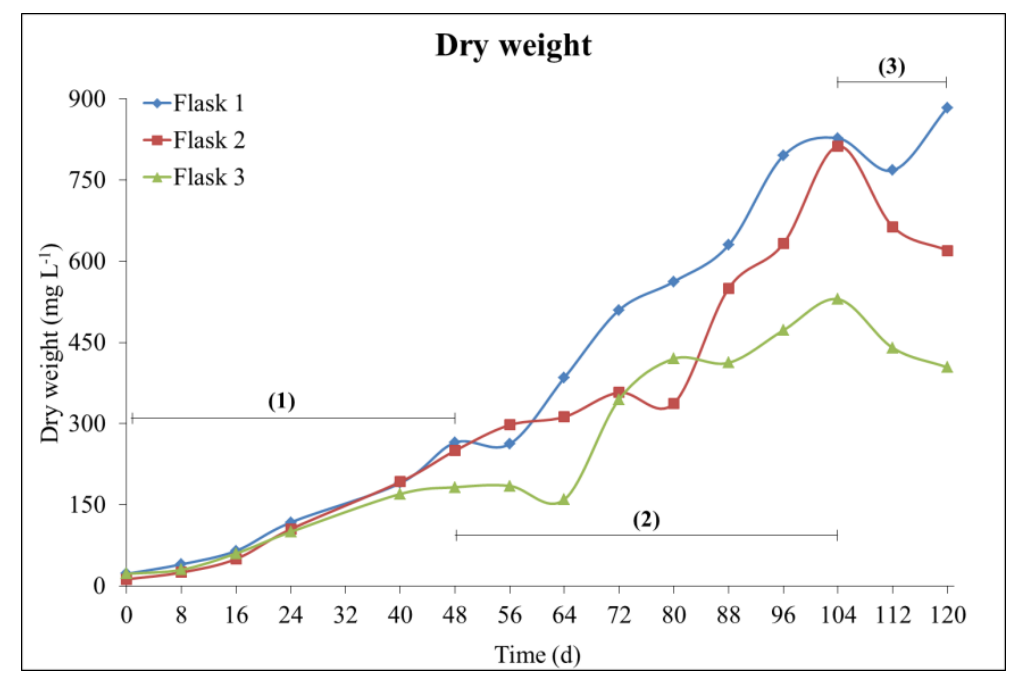

Figure 20: Dry weight of individual C. fritschii cultures during growth over 120 days under control conditions sampling every 8 days between 0 and 120 days, day 32 data omitted. Each data point is the dry weight value of each flask. Graph phases are represented by: (1) Phase 1, (2) phase 2 and (3) phase 3.

\section{Intracellular metabolite level changes}

GC-MS was used to analyse metabolite level changes during the different growth phases of $C$. fritschii over 120 days, sampling every 8 days. Due to the large amount of data produced over the time-series only identifiable peaks were investigated (match factor $\geq 60 \%$, level 2). A total of 369 were identified. One-way ANOVA revealed 277 peaks which were significantly different $(p<0.05)$ across the whole time-series with 95 of those remaining significant after BF correction. In general, the majority of identified peak levels reduced significantly comparing day 0 with each time point (t-Test $p<0.05$ ). Highest significant difference was calculated at day 80 (174 peaks) followed by day 112 (154 peaks) and day 120 (160 peaks). t-Test results between time points revealed highest significant differences in metabolite levels between days 32 and 40 with a total of 89 peaks. 48 of those reduced significantly with 41 increaing significantly. Comparing days 56 and 64, 79 peaks showed significant difference in normalised abundances with 39 peaks decreasing significantly and 40 increasing significantly in levels. Lowest significant difference was calculated between days 16 and 24 (total of 44 peaks) with 21 increasing significantly and 23 peaks reducing significsntly in levels.

PCA revealed a total variance of $37.3 \%(\mathrm{PC} 1=24.3 \%, \mathrm{PC} 2=7.7 \%$ and $\mathrm{PC} 3=5.3 \%)$ with good clustering of time points by replicates. Day 48 (sample 3) and day 104 
(sample 3) were omitted from analysis as anomalies. Separation can be seen across PC1 with increasing time. Separation between days 0 to 56 and 64 to 120 was observed (Figure 21).
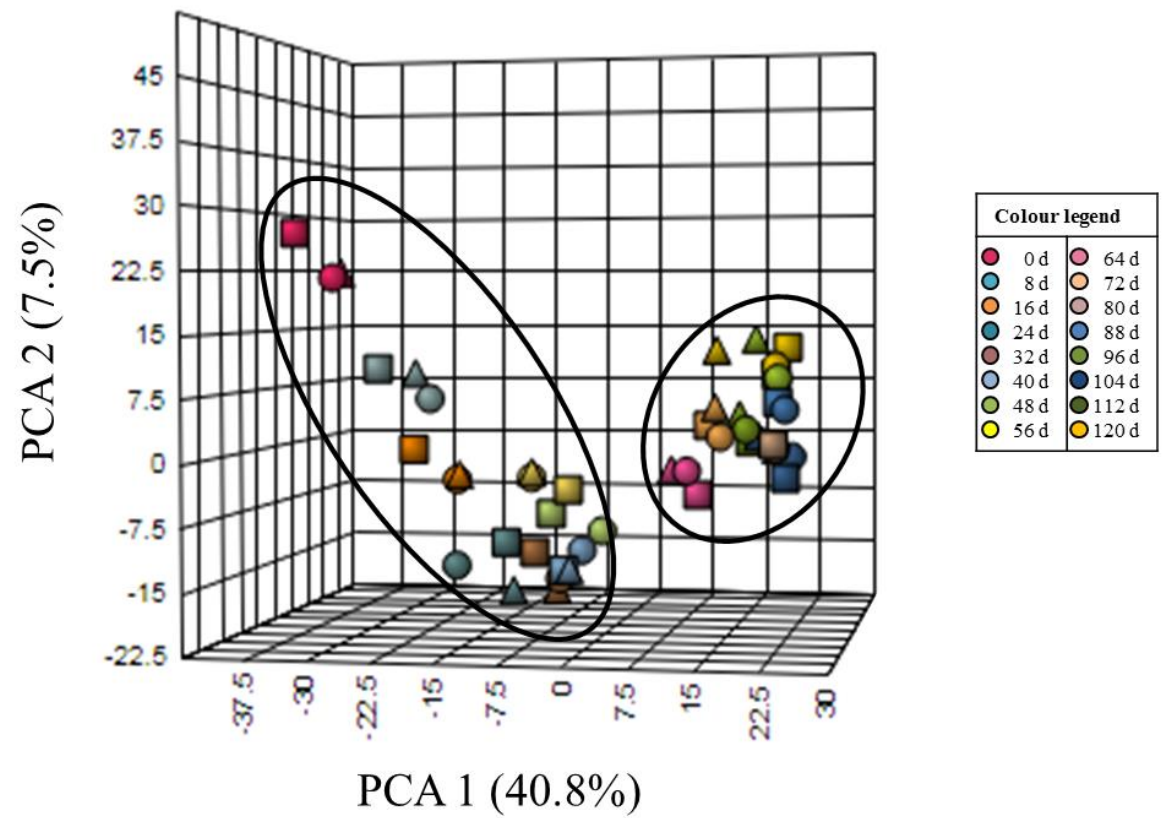

Figure 21: PCA of identified peaks (367) over 120 days from C. fritschii cultures. Samples from day 48 (S3) and 104 (S3) have been omitted. Data was normalised by sum, log transformed with Pareto scaling.

55 metabolites were identified overall with biological relevance to cyanobacterial metabolism (Table S3). Metabolites were classified according to chemical class as; acids, alcohols, amino acids, aromatics, fatty acids and sugars involved in a variety of metabolic pathways (Figure 22). The highest percentage of chemical class detected was acids (25\%). This included the four TCA metabolites fumarate, malate, citrate and succinate (Figure 22C). 

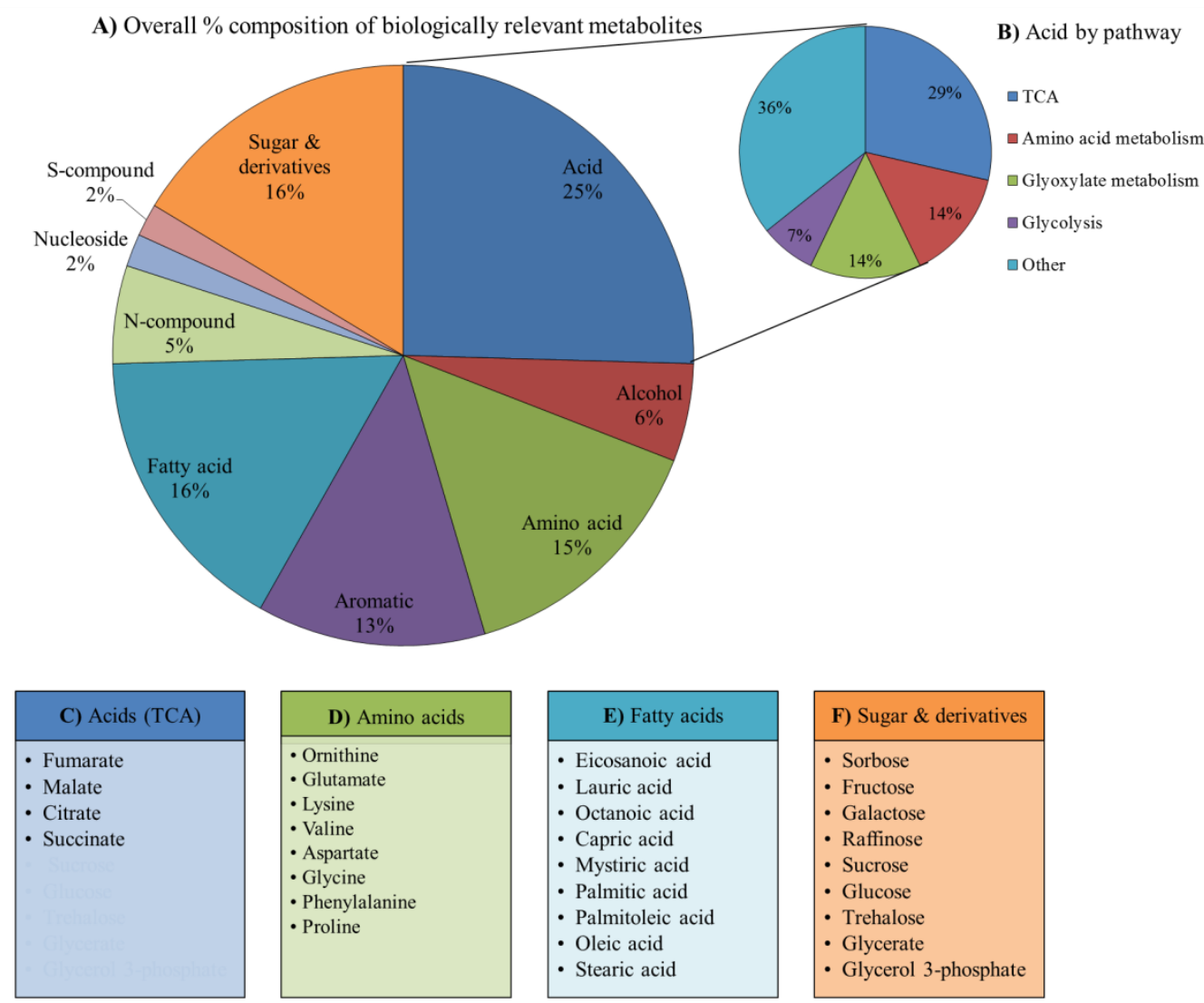

\begin{tabular}{|l|}
\hline F) Sugar \& derivatives \\
\hline - Sorbose \\
- Fructose \\
- Galactose \\
- Raffinose \\
- Sucrose \\
- Glucose \\
- Trehalose \\
- Glycerate \\
- Glycerol 3-phosphate \\
\hline
\end{tabular}

Figure 22: Overview of biologically relevant metabolites detected at each time-point combined, over the whole time-series of 120 days. A) Overall \% composition of detected metabolites by chemical class. B) Overall $\%$ of metabolites identified as acids by associated pathway. C, D, E and F) Identified metabolites by class as acids (involved in TCA), amino acids, fatty acids and sugars (and derivatives) over the whole time-series.

Overall, the number of detected biologically relevant metabolites increased with increasing time (Figure 23). Acids detected increased with time, with highest values at days 88, 112 and 120. The number of metabolites classified as alcohols remained steady throughout the time-series. The fatty acids detected increased at day 56 (9 detected) and remained consistent until the end of the time-series. Sugars and derivatives also increased in numbers with time with the exception of days 56 to 72 . Amino acids numbers varied with the highest numbers at day 32 (5) with a decrease in numbers until day 112, with (6 out of 8 ) detected (Figure 23). 


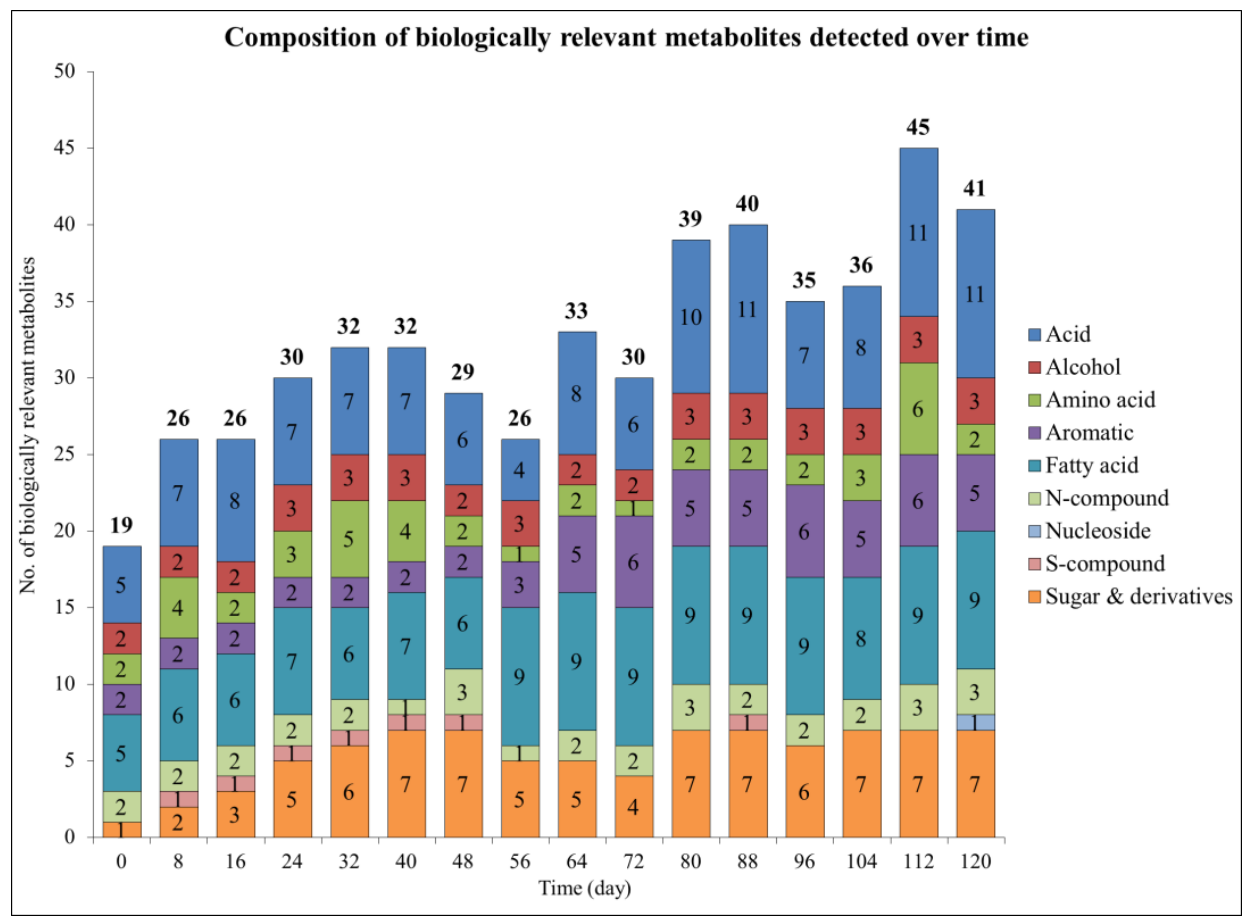

Figure 23: Composition of identified biologically relevant metabolites over time by chemical class.

45 biologically relevant metabolites were found to be significantly different after oneway ANOVA $(p<0.05)$ with 25 remaining significant after BF correction. Only $\sim 18 \%$ of metabolites were consistently identified across the whole time-series.

Malate and succinate, involved in the TCA cycle, showed an initial increase in levels on day 8 (0 vs $8 \mathrm{~d}, p<0.01)$ and day 16 ( 0 vs $16 \mathrm{~d}, p<0.001)$ respectively. This was followed by a decreasing trend over time Figure 24).
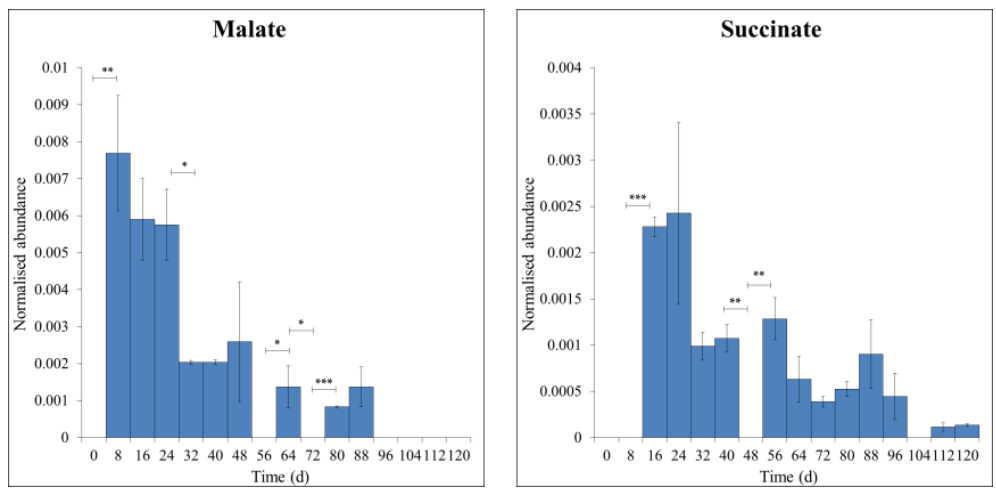

Figure 24: Metabolites involved in TCA cycle identified using GC-MS presented as mean values of normalised abundance \pm SE. Statistical significanct difference comparing day 0 to each time point (not displayed) as well as between time points was measured using a two sample t-Test with equal variance; $*=0.05>p \geq 0.01, * *=0.01>p \geq 0.001$ and $* * *=p<0.001$. 
Metabolites involved in amino acid biosynthesis (Figure 25) had varying detection and changes in levels over time. Glu increased significantly up to day 40 (0 vs 8, 16 and $40 \mathrm{~d}, p<0.05)$ whereas a significant decrease was measured up to 112 days ( 0 vs. 64, 72, 80, 88, 96 and $112 \mathrm{~d}, p<0.05$ ). Lysine (lys) accumulation was seen at day 104 (96 vs. $104 \mathrm{~d}, p<0.05$ ), with val levels increasing significant at days 40 (0 vs. $40 \mathrm{~d}$, $p<0.01)$ and 64 ( 0 vs. $64 \mathrm{~d}, p<0.05)$ followed by a decrease in levels with time (64 vs. $120 \mathrm{~d}, p<0.05)$. Asp levels decreased significantly (0 vs. $96 \mathrm{~d}, p<0.01)$. Phe increased in normalised abundance in the first 8 days ( 0 vs. $8, p<0.01$ ), no significant changes were calculated up to day 48; phe was not detected from day 56 to 120 . Benzoic acid was detected with accumulation at day 56 (48 vs. $56 \mathrm{~d}, p<0.05$ ) followed by a decrease in levels after 120 days (112 vs. $120 \mathrm{~d}, p<0.05)$.
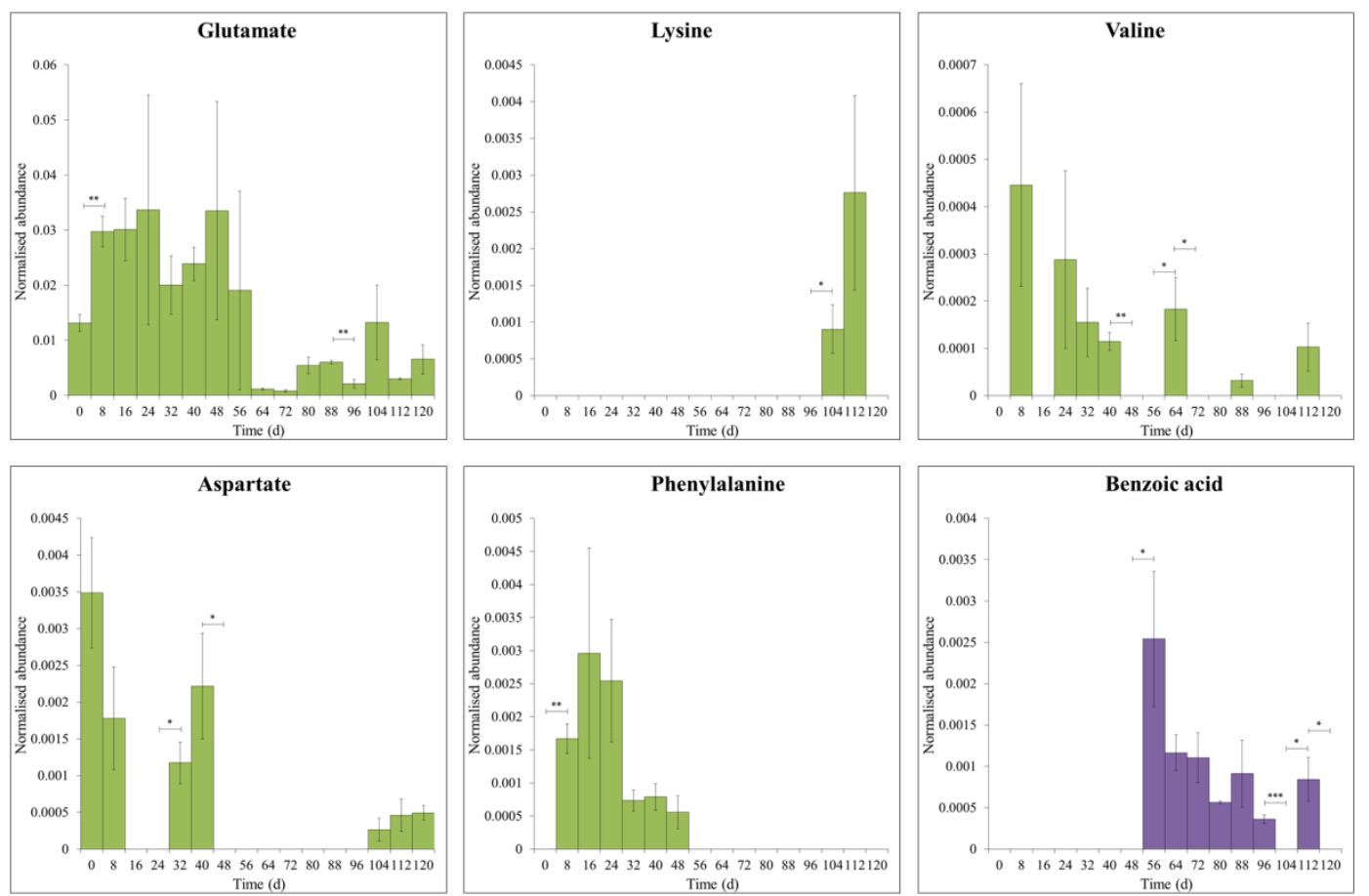

Figure 25: Metabolites involved in amino acid biosynthesis identified using GC-MS presented as mean values of normalised abundance \pm SE. Statistical significance comparing day 0 to each time point (not displayed) as well as between time points was measured using a two sample t-Test with equal variance; $*=0.05>p \geq 0.01, * *=0.01>p \geq 0.001$ and $* * *=p<0.001$.

The shorter chained saturated fatty acids (Figure 26); caprylic (C8:0) and capric (C10:0) were detected from day 56 only. They showed similar patterns of significant accumulation up to day 120 (0 vs. $120 \mathrm{~d}$, caprylic acid $p<0.05$, capric acid $p<0.001$ ). A similar pattern was observed for lauric acid (C12:0) but levels were detected during 
days 0 to 40, an overall decrease in levels was calculated ( 0 vs. $120 \mathrm{~d}, p<0.01)$. The longer chained saturated fatty acids (Figure 26); mystiric acid (C14:0), palmitic acid (C16:0), stearic acid (C18:0) and eicosanoic acid (C20:0) all showed similar patterns of decreasing levels ( 0 vs. $120 \mathrm{~d}, p<0.05)$. The unsaturated palmitoleic acid (C16:1) and oleic acid (C18:1) significantly increased in normalised abundance (0 vs $8 \mathrm{~d}$, $p<0.05$ and 0 vs. $24 \mathrm{~d}, p<0.05$ respectively).
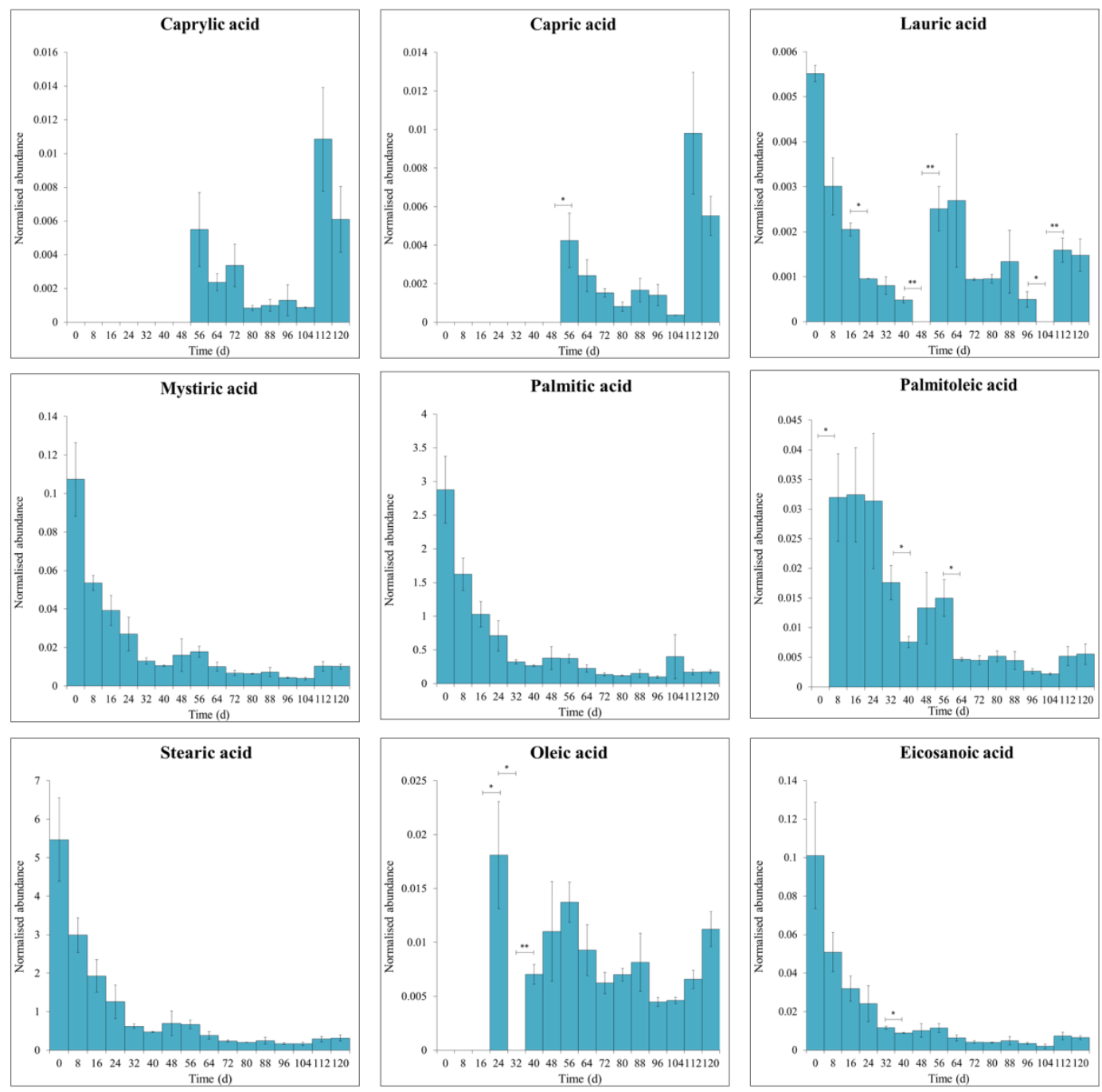

Figure 26: Metabolites involved in fatty acid biosynthesis identified using GC-MS presented as mean values of normalised abundance \pm SE. Statistical significance comparing day 0 to each time point (not displayed) as well as between time points was measured using a two sample t-Test with equal variance; $*=0.05>p \geq 0.01, * *=0.01>p \geq 0.001$ and $* * *=p<0.001$.

The sugar sorbose had an initial increase in levels ( 0 vs. $24 \mathrm{~d}, p<0.01)$ followed by a decrease after day 96 (96 vs. 104 d, $p<0.05$ ). Galactose also had an initial increase after 8 days ( 0 vs. $8 \mathrm{~d}, p<0.05)$ with an overall decrease in normalised abundance ( 0 vs. 32 
to $120 \mathrm{~d}, p<0.05)$. Fructose had an overall accumulation with increasing time (0 vs. $80,88,112$ and $120 \mathrm{~d}, p<0.01)$. Sucrose, raffinose and trehalose levels remained relatively consistent throughout. The alcohol glycerol showed decreasing levels throughout ( 0 vs. 8 to $120 \mathrm{~d}, p<0.01$ ) with glycerol 3-phosphate levels accumulating compared to day 0 ( 0 vs. 24, 32, 40, 72, 80, 96, 104 and $120 \mathrm{~d}, p<0.05$ ) (Figure 27).
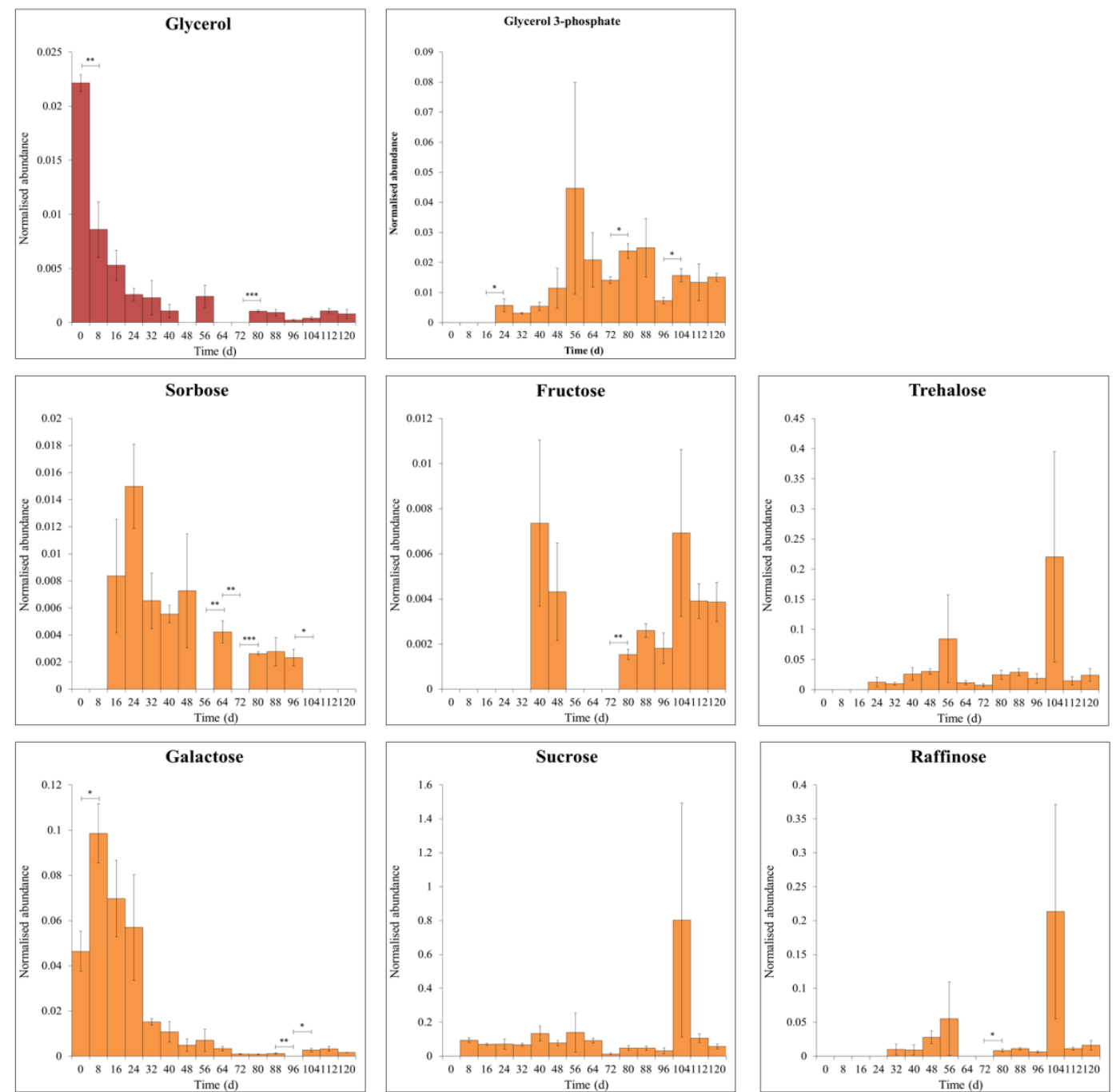

Figure 27: Metabolites involved in sugar biosynthesis identified using GC-MS presented as mean values of normalised abundance \pm SE. Statistical significance comparing day 0 to each time point (not displayed) as well as between time points was measured using a two sample t-Test with equal variance; $*=0.05>p \geq 0.01, * *=0.01>p \geq 0.001$ and $* * *=p<0.001$. 


\subsubsection{Time-series analysis of $C$. fritschii over $48 \mathrm{~h}$}

A time-series analysis over $48 \mathrm{~h}$ was then undertaken to analyse the short-term changes in metabolite levels as measured by GC-MS. This experiment was also used as a control for standard growth conditions (PAR only) comparing to the UV exposure experiments (UV-B + PAR and UV-A + PAR, Chapter 3).

The dry weight (Figure 28) during the time-series was measured and an increase was observed after $48 \mathrm{~h}$ with a significant increase as measured by a two-sample t-Test $(p<0.05,0$ vs. $24 \mathrm{~h})$.

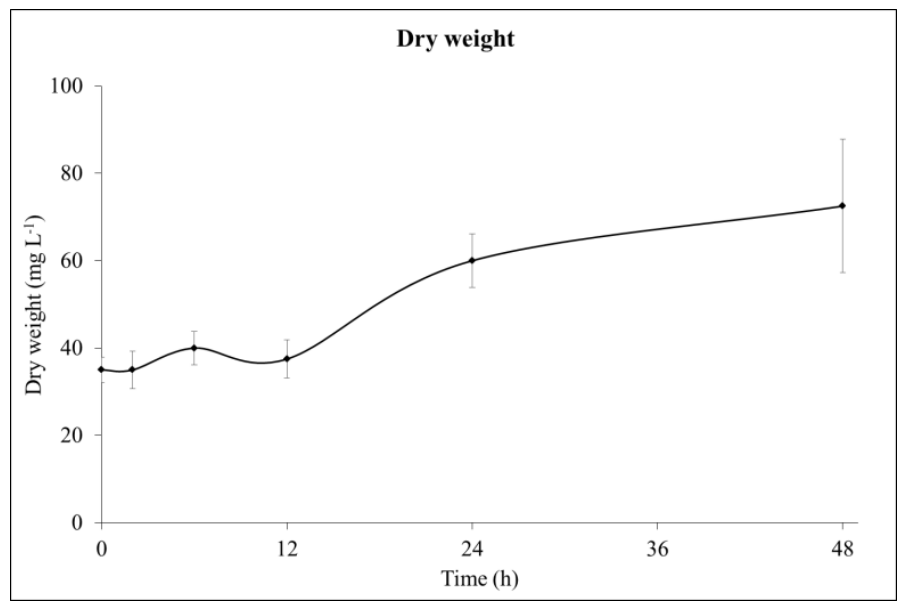

Figure 28: Dry weight of $C$. fritschii cultures ( $\mathrm{n}=3$ ) during growth over $48 \mathrm{~h}$ under control conditions sampling at $0,2,6,12,24$ and $48 \mathrm{~h}$. Each data point is the mean value of biological triplicates \pm SE.

\subsubsection{Intracellular metabolite level changes}

At each time point, samples were extracted using the optimised extraction protocol above (Section 2.2.1) and analysed using GC-MS. 169 peaks were detected with 137 peaks identified as 'true hits.' Of those 137 peaks, 70 were putatively identified (match factor $\geq 60 \%$, level 2) and 67 left unassigned (match factor $<60 \%$, level 4 ).

A two-sample t-Test with equal variance was used to evaluate the significant differences of normalised metabolite level changes comparing $0 \mathrm{~h}$ to each time-point $(2,6,12,24$, and $48 \mathrm{~h})$. Overall 76 peaks showed significance $(p<0.05)$ between one or more time point with 22 peaks remaining significant $(p<0.05)$ after BF correction. Twenty-four (24) $\mathrm{h}$ had the highest significant difference with 35 statistically significant peaks ( 0 vs. $24 \mathrm{~h}, p<0.05$ ), $71 \%$ of those had elevated levels (positive log2 FC) whereas $29 \%$ reduced in levels (negative $\log 2 \mathrm{FC}$, Table 19). This is consistent 
with the dry weight values (Figure 28) where a significant increase in biomass was observed after $24 \mathrm{~h}(0$ vs. $24 \mathrm{~h}, p<0.05)$.

Table 19: Comparison of significantly different $(p<0.05)$ peaks (out of 137) and number of those significantly different peaks with a positive or negative $\log 2 \mathrm{FC}$ during PAR only exposure. A positive $\log 2 \mathrm{FC}$ indicates an increase in metabolite level and a negative $\log 2 \mathrm{FC}$ value indicates a decrease in metabolite level. Both significance and $\log 2 \mathrm{FC}$ was calculated comparing control $(0 \mathrm{~h})$ and 2, 6, 12, 24 and $48 \mathrm{~h}$ of PAR only exposure.

\begin{tabular}{cccc}
\hline $\begin{array}{c}\text { PAR only } \\
\text { (h) }\end{array}$ & $\begin{array}{c}\text { No. of peaks, } \\
\boldsymbol{p}<\mathbf{0 . 0 5}^{\mathbf{a}}\end{array}$ & $\begin{array}{c}\text { No. of peaks, } \\
\text { positive log2FC }\end{array}$ & $\begin{array}{c}\text { No. of peaks, } \\
\text { negative log2FC }\end{array}$ \\
\hline 0 vs. $\mathbf{2}$ & 20 & 18 & 2 \\
0 vs. 6 & 11 & 4 & 7 \\
0 vs. $\mathbf{1 2}$ & 26 & 21 & 5 \\
0 vs. $\mathbf{2 4}$ & 35 & 25 & 10 \\
0 vs. $\mathbf{4 8}$ & 32 & 24 & 8 \\
\hline
\end{tabular}

${ }^{\mathrm{a}}=$ Significant difference was calculated using a two-sample t-Test with equal variance.

38 key primary metabolites were detected and identified during the time-series (20 highlighted for simplicity, Figure 29) which are involved in cyanobacterial metabolism including; the pentose phosphate pathway, glycolysis, TCA, amino acid and fatty acid biosynthesis (Table S4). 


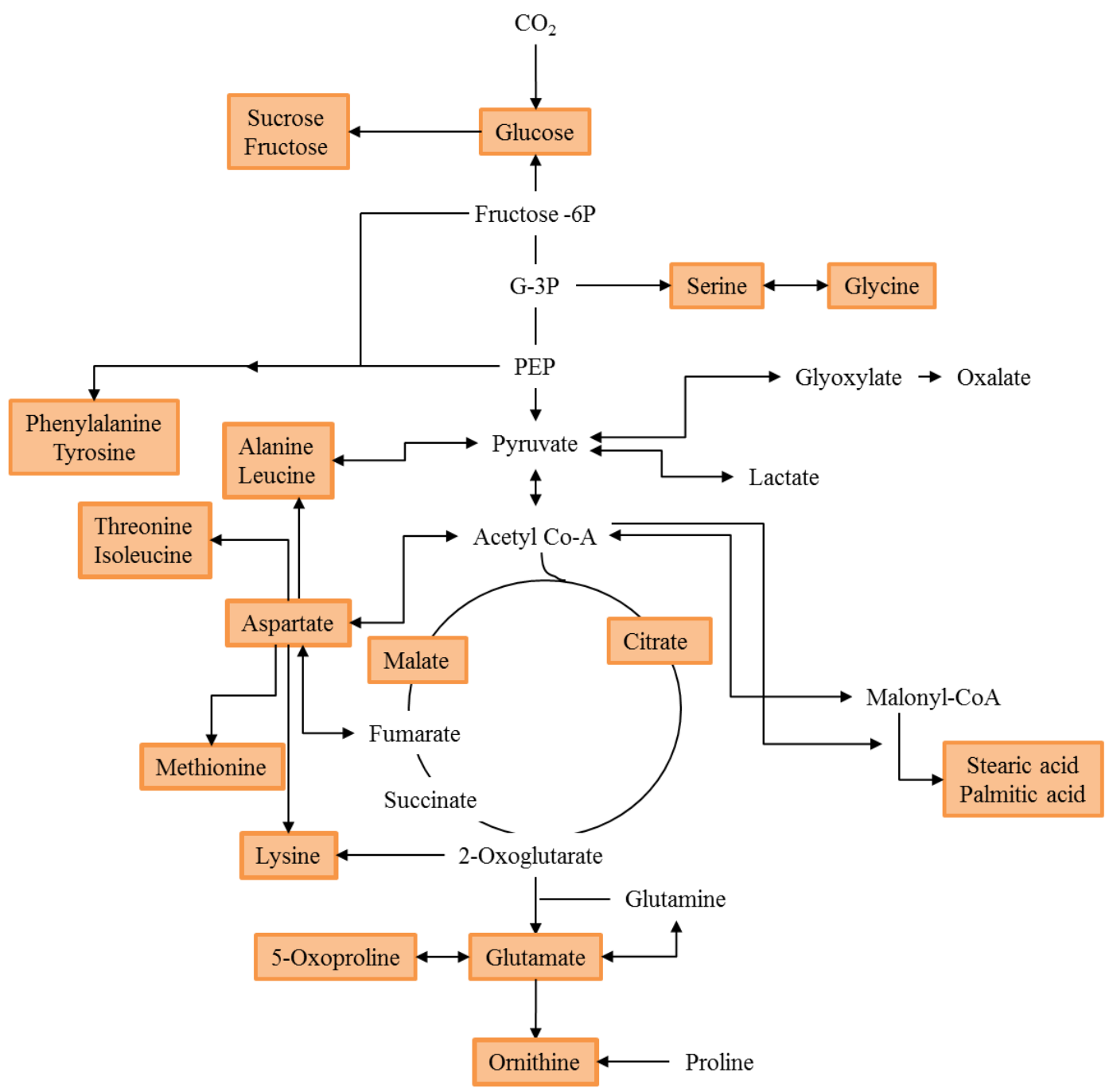

Figure 29: Schematic representation of a generalised reduced carbon metabolism in the cyanobacterium C. fritschii showing glycolysis, TCA cycle, amino acid and fatty acid biosynthesis. Primary metabolites identified in intracellular samples using GC-MS are highlighted in orange.

12 proteinogenic amino acids were detected during the $48 \mathrm{~h}$ time series. Five amino acids showed statistically significant accumulation; glu ( 0 vs. $12, p<0.01 ; 0$ vs. 24 h, $p<0.05$; 0 vs. $48 \mathrm{~h} p<0.05$ ), methionine (met, 0 vs. $12 \mathrm{~h}, p<0.001$ ), asp (0 vs. $48 \mathrm{~h}$, $p<0.05)$, tyr ( 0 vs. $2 \mathrm{~h}, p<0.01 ; 0$ vs. $12 \mathrm{~h}, p<0.001 ; 0$ vs. $24 \mathrm{~h}, p<0.05 ; 0$ vs. $48 \mathrm{~h}$ $p<0.001)$ and lys ( 0 vs. 2 h, $p<0.05 ; 0$ vs. 12 h, $p<0.001 ; 0$ vs. 24 h, $p<0.05 ; 0$ vs. 48 h $p<0.001$ ). Tyr and lys also showed significance after BF correction (Tyr: 0 vs. $12 \mathrm{~h}$, BF $p<0.05$; 0 vs. 48 h, BF $p<0.001$ and lys 0 vs. 48 h, BF $p<0.01$ ).

Three of the 12 amino acids showed decreasing levels throughout the time series comparing $0 \mathrm{~h}$ with each time point including; phe $(0$ vs. $24 \mathrm{~h}, p<0.01 ; 0$ vs. $48 \mathrm{~h}$, $p<0.05)$, leu ( 0 vs. $24 \mathrm{~h}, p<0.05)$ and ile (0 vs. $24 \mathrm{~h}, p<0.01)$. 
Ala, thr, ser and gly showed steady metabolite levels with no statistical significance $(p>0.05)$.
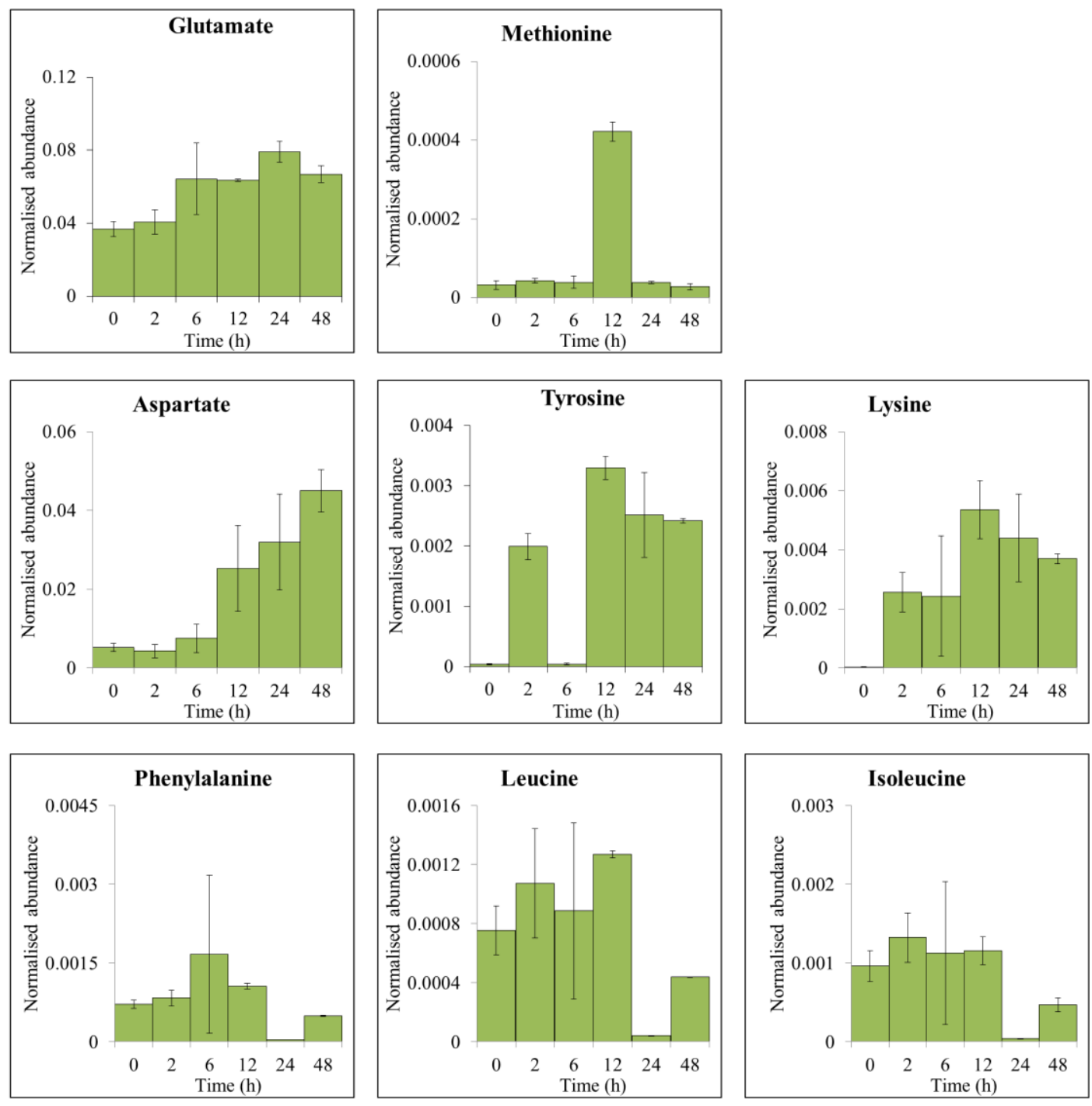

Figure 30: Metabolites involved in amino acid biosynthesis identified using GC-MS presented as mean values of normalised abundance \pm SE. Statistical significance comparing $0 \mathrm{~h}$ to each time point as well as between time points was measured using a two sample t-Test with equal variance; $*=0.05>p \geq 0.01$, $* *=0.01>p \geq 0.001$ and $* * *=p<0.001$.

Six sugars were identified during the time-series (Figure 31), glucose accumulation was seen after 2 h ( 0 vs. 2 h, p<0.01) with a decrease in levels after 24 h ( 2 vs. 24 h, $p<0.01)$. Sorbose levels were similar to glucose where an accumulation was observed after $2 \mathrm{~h}$ followed by a decrease after $24 \mathrm{~h}(0$ vs. $2 \mathrm{~h}, p<0.05 ; 2$ vs. $24 \mathrm{~h}, p<0.05)$. Sucrose accumulated after $2 \mathrm{~h}(0$ vs. $2, p<0.01)$ with levels remaining steady with increasing time. Rhamnose showed a decrease in normalised abundance after 6 h, 24 $\mathrm{h}$ and $48 \mathrm{~h}$ which remained significant after BF correction ( 0 vs. $6, p<0.001 ; 0$ vs. 24 , 
$p<0.001 ; 0$ vs.48 h, $p<0.001)$. Fructose and trehalose levels remained constant throughout with no significant changes $(p>0.05)$. The sugar-phosphate, mannose-6phosphate accumulated after 24 and $48 \mathrm{~h}$ and also remained significant after BF correction after $48 \mathrm{~h}(0$ vs. $24, p<0.05 ; 0$ vs. 48 h, $p<0.001$, BF $p<0.01)$.

The sugar acid glycerate increased in levels after 2 and $12 \mathrm{~h}(0$ vs. $2, p<0.01 ; 0$ vs. 12 , $p<0.001$, BF $p<0.05)$. Glycerol-3-phosphate, a phosphoric ester of glycerol, decreased in normalised abundance after 24 and $48 \mathrm{~h}$ ( 0 vs. $24 \mathrm{~h}, p<0.05 ; 0$ vs. $48 \mathrm{~h} p<0.05)$.
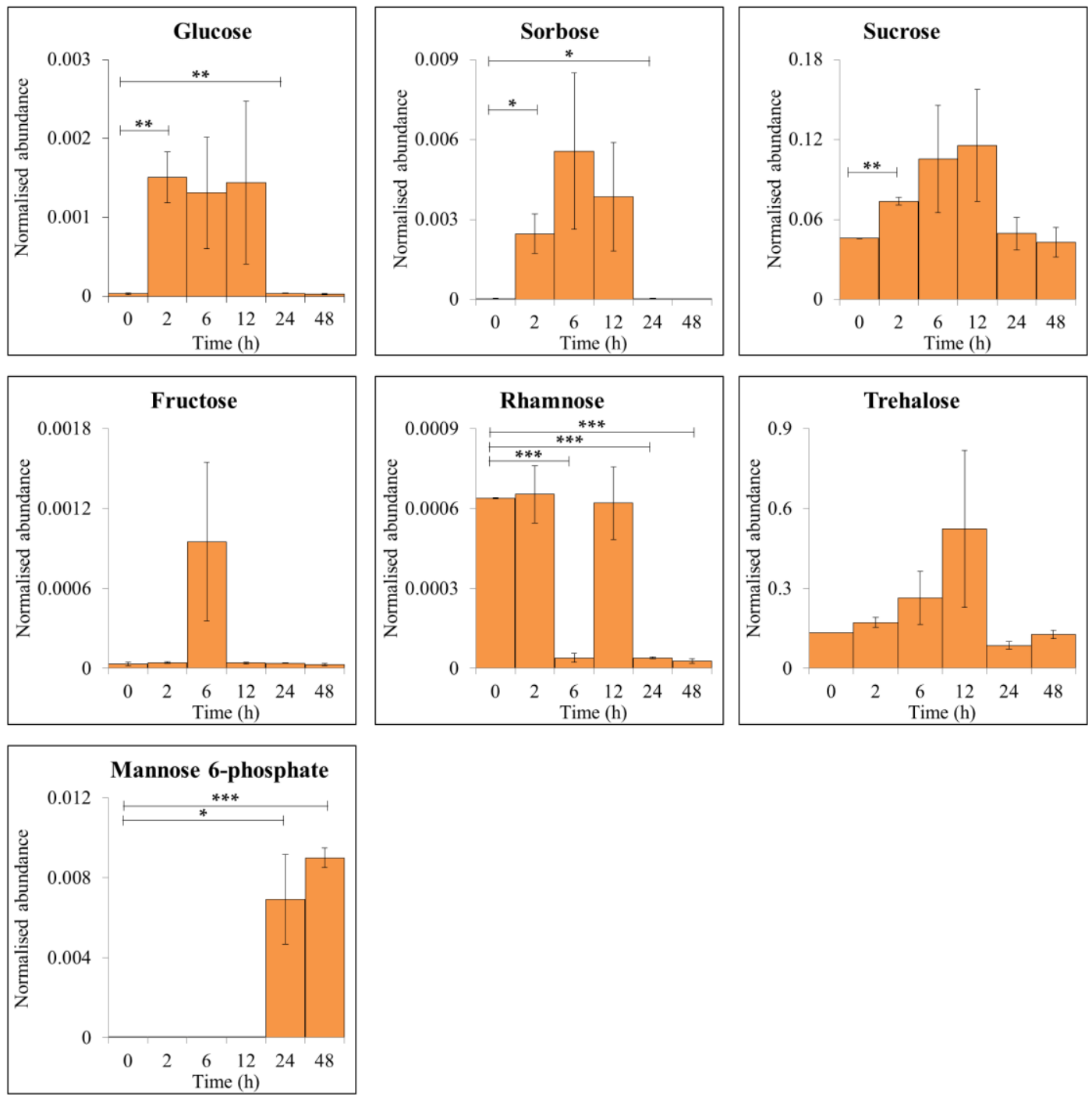

Figure 31: Metabolites involved in sugar biosynthesis identified using GC-MS presented as mean values of normalised abundance \pm SE. Statistical significance comparing $0 \mathrm{~h}$ to each time point as well as between time points was measured using a two sample $\mathrm{t}$-Test with equal variance; $*=0.05>p \geq 0.01$, $* *=0.01>p \geq 0.001$ and $* * *=p<0.001$. 
Two metabolites were identified as being involved in TCA cycle (Figure 32), a significant accumulation of malate was seen after 48 h ( 0 vs. 48 h, $p<0.01)$ whereas no significant changes in citrate levels were observed $(p>0.05)$.
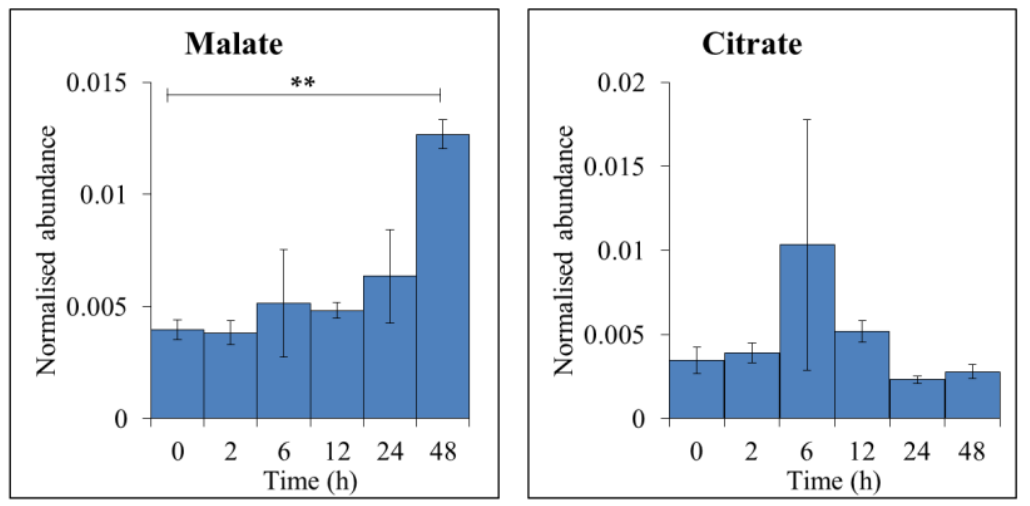

Figure 32: Metabolites involved in the TCA cycle identified using GC-MS presented as mean values of normalised abundance \pm SE. Statistical significance comparing $0 \mathrm{~h}$ to each time point as well as between time points was measured using a two sample t-Test with equal variance; $*=0.05>p \geq 0.01$, ** $=0.01>p \geq 0.001$ and $* * *=p<0.001$.

The fatty acids stearic and palmitic acid (Figure 33) had steady levels throughout with no significance $(p>0.05)$.
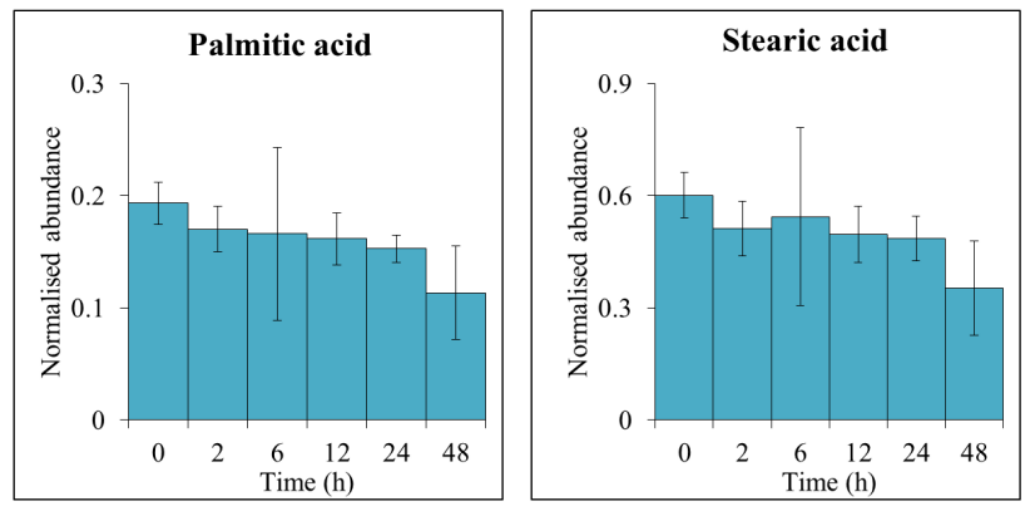

Figure 33: Metabolites involved in fatty acid biosynthesis identified using GC-MS presented as mean values of normalised abundance \pm SE. Statistical significance comparing $0 \mathrm{~h}$ to each time point as well as between time points was measured using a two sample t-Test with equal variance; $*=0.05>p \geq 0.01$, $* *=0.01>p \geq 0.001$ and $* * *=p<0.001$. 


\subsection{Discussion}

This chapter presents an overview of the metabolites detected and identified using GCMS over a variety of time-series during standard growth conditions $\left(27{ }^{\circ} \mathrm{C}\right.$ with continuous PAR at $15 \mu \mathrm{mol} \mathrm{m} \mathrm{m}^{-1} \mathrm{~s}^{-1}$. Metabolite level changes were investigated and the differences between axenic and xenic cultures were explored.

Firstly, the extraction protocol was investigated. Unbiased characterisation of metabolites is difficult within metabolomics due to the chemical complexity within biological systems [45]. Results showed that solvent type was the main factor to consider when extracting metabolites. The more polar solvent $\mathrm{MeOH}$ extracted polar metabolites and non-polar $\mathrm{CHCl}_{3}$ extracting non-polar metabolites. These results confirmed that $\mathrm{CHCl}_{3}: \mathrm{MeOH}$ was the best combination of solvent to extract the most variety of metabolites. By adding $\mathrm{H}_{2} \mathrm{O}$, phase separation was possible to easily decant the same volume of both layers for GC-MS analysis, this step was added in further experimental extractions. For cyanobacteria, a suitable disruption technique is also required to break down the cell wall. Analysis of sonicated samples showed increased number of detected peaks throughout. Compared to the other disruption techniques (ice-bath and freeze-thaw), sonication was seen as a more aggressive form of disruption to break down the cell wall. As consistency is important in metabolomics analysis, the same procedure was used in all subsequent experiments using $\mathrm{CHCl}_{3}: \mathrm{MeOH}: \mathrm{H}_{2} \mathrm{O}$ with sonication (probe).

During this research, one of the main bottlenecks was the low biomass production and difficulty in maintaining axenic $C$. fritschii cultures. For the true metabolome to be identified, monocultures need to be analysed. In industrial biotechnology, cultivation of cyanobacteria and other microalgal species at large scale increases the risk of contamination. Cyanobacteria form symbiotic relationships with other bacteria that can increase growth and has potential in biotechnology by producing novel industrially relevant metabolites [4]. Exclusion of bacterial communities would not reflect the situation more closely related to their natural environment and could cause additional stress [94]. Metabolite production in both axenic and xenic cultures was therefore examined to observe any differences in growth, bulk carbohydrate, protein and metabolite identification over 28 days of growth. 
Initial biomass concentration was greater in xenic cultures due to their higher productivity in pre-experimental cultivation. Growth rate showed differences up to day 8 but were similar between the two cultures from day 8 to 28 (Figure 13 and Figure 14). Carbohydrate levels also differed up to day 8 with no significance observed in protein levels. Although axenic cultures detected more peaks overall during GC-MS analysis, $73 \%$ of biologically relevant metabolites were identified in both cultures. These metabolite levels differed significantly between the first 4 days (axenic vs. xenic) but significance reduced with increasing time. Overall composition was very similar between the two with differences including the amino acids ile, tyr (axenic only) and ala (xenic only) as well as the sugars mannose, fructose and galactose (axenic only) and glucose (xenic only). These differences were due to missing data points in either axenic or xenic cultures. Missing values in GC-MS analysis is a common drawback [103]. Within these biological experiments, missing values are assumed to be below detection limit.

The overall similarity in both cultures could indicate the low impact of additional microorganisms to the culture after the first 8 days with $C$. fritschii remaining the dominant species within the flask (evaluated using microscopic analysis). Variation in the first 8 days could be due to the inevitable varying growth of $C$. fritschii from separate cultures. These results could also indicate the contamination of axenic cultures after day 8. Further work into the characterisation of the specific microorganisms present within $C$. fritschii cultures, using a combination of metabolomics, metagenomics and transcriptomics [4], should be considered followed by controlled co-culture experiments to determine contributions to the metabolites detected [104].

Cyanobacterial growth relies on light to drive photosynthesis and nutrient levels and space within culture vessels. Typical growth profiles consist of an initial lag phase, exponential phase (cell proliferation), stationary phase, and declining phase [105]. Metabolites have varying levels during these different growth phases as seen in the diatom Skeletonema marinoi [94], green microalga Chlorella sorokiniana [106] the marine roseobacter Pharobacter gallaeciensis DSM 17395 [107] and the cyanobacterium Synechococcus elongates PCC 7942 [108]. 
Increasing SE bars can be explained by the inconsistent dry weight measurements between the replicate flasks (Figure 20) where flask 3 began to decline and had lower dry weight values overall after day 40 .

A pattern between days 0 to 48 (phase 1), 56 to 104 (phase 2) and 112 to 120 (phase 3) can be seen within the growth curve (Figure 19 and Figure 20). This could correspond to the different cyanobacterial growth phases as lag, exponential and stationary phases. The composition of biologically relevant metabolites detected over the whole time-series (Figure 23) also shows a pattern associated with this grouping of days. This can also be seen within individual metabolite level changes such as; succinate (Figure 24), phe, benzoic acid (Figure 25), caprylic aicd, capric acid and lauric acid (Figure 26).

In lag phase, growth is low while cells prepare for exponential phase. TCA substrates and amino acids (glu, val, asp and phe) accumulate to prepare for cell proliferation [108]. Amino acids are required for protein synthesis and formation of nitrogen containing metabolites as indicated by a decrease in levels. Phe was undetected after day 48 , possibly due to the conversion to benzoic acid, a precursor to many essential compounds and natural products, which accumulated at day 56 [109].

Many identified metabolites have potential uses in biotechnology including glycolic acid, lactic acid, pyruvic acid, succinate, malate and fumarate and adipic acid which can be expoited as platform chemicals [110]. Bioactive metabolites will be explored further in Chapter 5.

As seen in the axenic vs. xenic experiment, many metabolites were not identified within all time-points. Intracellular metabolites have a high turnover rate. With a gap of 8 days between each sampling point much biological data is lost and difficulties are presented in biological interpretation. Sampling more frequently within this time frame of 8 days would be more beneficial, increasing sampling points increases the complexity of data analysis as seen within this 120-day experiment. From an industrial biotechnology perspective, the identification of a variety of metabolites is essential for understanding the production of potential relevant metabolites for industry over the growth of $C$. fritschii and for further investigations during stress conditions which will be discussed in more detail throughout the remaining chapters. This study presents the 
first metabolomic data set for $C$. fritschii as measured by GC-MS over a long growth period and a good base platform for further systems biology investigation.

Time-series metabolomics is becoming more common to evaluate changes in metabolite levels over time and to allow better insight into the physiology of the cells [111]. As seen in the previous experiment the correct sampling points are needed for relevant biological interpretation due to the high turnover rates of intracellular processes.

The limited statistical significance in metabolite levels reflects the slow growth of $C$. fritschii over a short time-series of $48 \mathrm{~h}$ indicating little changes in metabolism during standard growth conditions. The majority of significant metabolites increased in levels preparing cells for further exponential growth (Table 19) [108].

This experimental analysis will be used as a control for the UV exposure experiments in Chapter 3.

\subsection{Conclusion}

During the course of this research, a more realistic approach was taken to use unialgal cultures (other microorganisms may be present in small amounts), because the presence of other microorganisms would have a limited effect on the metabolites produced as seen in the axenic vs. xenic experiment and would not produce the chosen targeted metabolites MAAs (target analysis in Chapter 3 and 4).

Metabolite level changes during $48 \mathrm{~h}$ showed little significant differences as expected in this short timescale. Combination of long-term analysis with shorter term samplings at each growth phase would give a better insight in the changes in metabolite levels. Analysing the metabolite levels can help to characterise the observable phenotype and production of useful industrially relevant metabolites over a time-series. This data can be used to further investigate $C$. fritschii with other-omic techniques including fluxomics for potential quantitative analysis of value-added chemicals. 


\section{Chapter 3: Intracellular and Extracellular Metabolite Level Changes of Chlorogloeopsis fritschii, PCC 6912, During Ultraviolet (UV) A and UV-B Exposure}

Aims

- To optimise a HPLC method (mobile and stationary phase optimisation) for the analysis of MAAs using macroalgal extracts with known MAA compositions.

- To analyse the intracellular untargeted metabolite profile of $C$. fritschii using GCMS during supplemented UV-B (PAR + UV-B) or UV-A (PAR + UV-A) over a $48 \mathrm{~h}$ time-series.

- To analyse extracellular low molecular weight compounds from $C$. fritschii using GC-MS during supplemented UV-B over a $48 \mathrm{~h}$ time-series. 


\section{Introduction}

The effect of UV radiation on cyanobacteria has been widely researched including the interaction with biomolecules, production of ROS which cause oxidative stress, impaired growth, partial inhibition of photosynthesis and decreased enzyme activity $[24,25,112]$. UV radiation also has a role as an activator of secondary metabolite production such as MAAs [23] and other protective secondary metabolites [28]. Many studies have been conducted to identify these targeted intracellular metabolites during UV-B and UV-A exposure in Lyngbya sp. CU2555 [49], Nostoc commune [64], Anabaena variabilis PCC 7937 [65], Calothrix sp. [10] and C. fritschii PCC 6912 [48] to name a few. Other studies conducted have sought to evaluate changes at the protein level [113,114], targeted and untargeted metabolomic analysis using different intensities of UV-B [99] and combined metabolomic and proteomic analysis during UV-A exposure [52].

Cyanobacteria convert $\mathrm{CO}_{2}$ into reduced carbon which forms the backbone of metabolites and are central to life. Like many other microorganisms, cyanobacteria release these carbon-based primary and secondary metabolites into their surrounding area which drives carbon cycling within microbial communities [54,115]. These released metabolites are by-products of metabolism within cells and make up a small proportion of the dissolved organic matter (DOM) pool within freshwater and marine ecosystems [54]. Consisting of a variety of chemical compositions such as; polysaccharides, proteins, lipids, organic compounds or inorganic molecules, they are released for communication, structural organisation, and defence against biotic and abiotic factors [115-117]. The uptake and release of metabolites change with varying environments; examples include the release of exopolysaccharides during high light and UV radiation $[64,118]$.

Monitoring industrially relevant metabolites released by microorganisms into their surroundings is a widely used technique in the fermentation industry. It can be used in bioprocess monitoring, fermentation biomarker identification, for monitoring metabolite levels in fermentation processes and microbial contamination $[68,69]$.

Combining intracellular and extracellular analysis is useful in the study of cyanobacteria providing a more holistic picture of metabolite production during growth and its response to different environmental conditions [69,70]. Metabolite 
profiling can be used to view the physiological changes at the metabolite level in response to changing environments and is a useful tool in cyanobacterial research [43].

Little work has been undertaken on monitoring both intracellular and extracellular metabolites in cyanobacteria. It is important to investigate the changes in primary metabolites in order to understand secondary metabolite production. Manipulating abiotic stress such as UV radiation can be used to enhance production of useful metabolites for uses in industrial biotechnology [16,21,22].

In this chapter, we observe the changes in metabolites produced by $C$. fritschii during $48 \mathrm{~h}$ of UV-A and UV-B exposure as detected by GC-MS. We were able to identify metabolites with altered levels comparing UV-B (PAR + UV-B) and UV-A (PAR+UV-A) treatments to cultures irradiated with PAR only (Chapter 2). 


\subsection{Experimental design}

\subsubsection{MAA HPLC method development}

See Section 1.4 for the HPLC method development for the detection of MAAs.

\subsubsection{UV-B exposure over $48 \mathrm{~h}$}

After 6 days of pre-growth (Section 1.1), triplicate experimental $C$. fritschii cultures were transferred into three Quartz Erlenmeyer flasks (H. Baumbach \& CO. LTD, Suffolk) at an $\mathrm{OD}_{750 \mathrm{~nm}}$ of approx. 0.14 to allow even UV-B exposure. The cultures were exposed to a total of $48 \mathrm{~h}$ of UV-B radiation using a UVB broadband (290-315 $\mathrm{nm}$, centered at $310 \mathrm{~nm}$ ) fluorescent tube (Philips TL 20W/12 RS SLV/25, Proflamps) emitting $3 \mu \mathrm{mol} \mathrm{m} \mathrm{m}^{-2} \mathrm{~s}^{-1}$ of UV-B radiation (measured using a UVR light sensor, Enviromonitors, UK). The experiment was carried out under continuous PAR at 15 $\mu \mathrm{mol} \mathrm{m} \mathrm{m}^{-2} \mathrm{~s}^{-1}$ and shaking at $100 \mathrm{rpm}$ for even UV-B exposure of cells. For time- series analysis samples were harvested at no UV-B $(0 \mathrm{~h}), 2,6,12,24$ and $48 \mathrm{~h}$ for dry weight (Section 1.2), pigment (Section 1.5.1), MAA (Section 1.4.2-1.4.3) and GC-MS analysis (Sections 1.3.2 - 1.3.6).

\subsubsection{UV-A exposure over $48 \mathrm{~h}$}

After 6 days of pre-growth (Section 1.1), triplicate experimental C. fritschii cultures were transferred into three Quartz Erlenmeyer flasks (H.Baumbach \& CO.LTD, Suffolk) at an $\mathrm{OD}_{750 \mathrm{~nm}}$ of approx. 0.16 to allow even UV-A exposure. UV-A was supplied by a Philips TL-D $18 \mathrm{~W}$ black light blue fluorescent tube (315-400 nm, centred at $360 \mathrm{~nm}$ ) emitting $11 \mu \mathrm{mol} \mathrm{m} \mathrm{m}^{-2} \mathrm{~s}^{-1}$ (measured using a UVR light sensor, Enviromonitors, UK). The experiment was carried out under continuous PAR at 15 $\mu \mathrm{mol} \mathrm{m} \mathrm{m}^{-2} \mathrm{~s}^{-1}$, a temperature of $27 \pm 2{ }^{\circ} \mathrm{C}$ and shaking at $100 \mathrm{rpm}$ for even UV-A exposure of cells. For time-series analysis samples were harvested at no UV-A $(0 \mathrm{~h})$, 2, 6, 12, 24 and $48 \mathrm{~h}$ for dry weight (Section 1.2), pigment (Section 1.5.1), MAA (Section 1.4.2-1.4.3) and GC-MS analysis (Sections 1.3.2 - 1.3.6). 


\subsection{Results}

\subsubsection{Method development for the detection of mycosporine-like amino acids (MAAs) using High Performance Liquid Chromatography (HPLC).}

Method optimisation for extracts with known MAA content was evaluated using the macroalgae, P. palmata due to its accumulation of the MAAs; shinorine, pathythine, porphyra, usijurene and palythene in high concentrations under natural sunlight. This optimised method was then used for the detection of MAAs in C. fritschi in subsequent experiments.

\subsubsection{Mobile and stationary phase optimisation}

TFA is a common ionic mobile phase additive, three concentrations were chosen to evaluate the lowest amount needed for good separation of peaks by calculating resolution (Rs, Figure 34A) and repeatability using relative standard deviation between technical replicates (RSD \%, Figure 34B). The results showed little difference between $0.06 \%$ and $0.01 \%$ TFA with a higher Rs between peaks 3 and 4 (asterina and porphyra-334 respectively) using 0.06\% TFA and a higher Rs between 4 and 5 (porphyra-334 and palythinol respectively) using 0.01\% TFA. The lowest Rs was seen using $0.001 \%$ TFA. The RSD\% between replicates $(n=5)$ showed $0.01 \%$ TFA to have better repeatability. A concentration of $0.01 \%$ was therefore chosen as all of the peaks were eluted with a good separation and good repeatability.

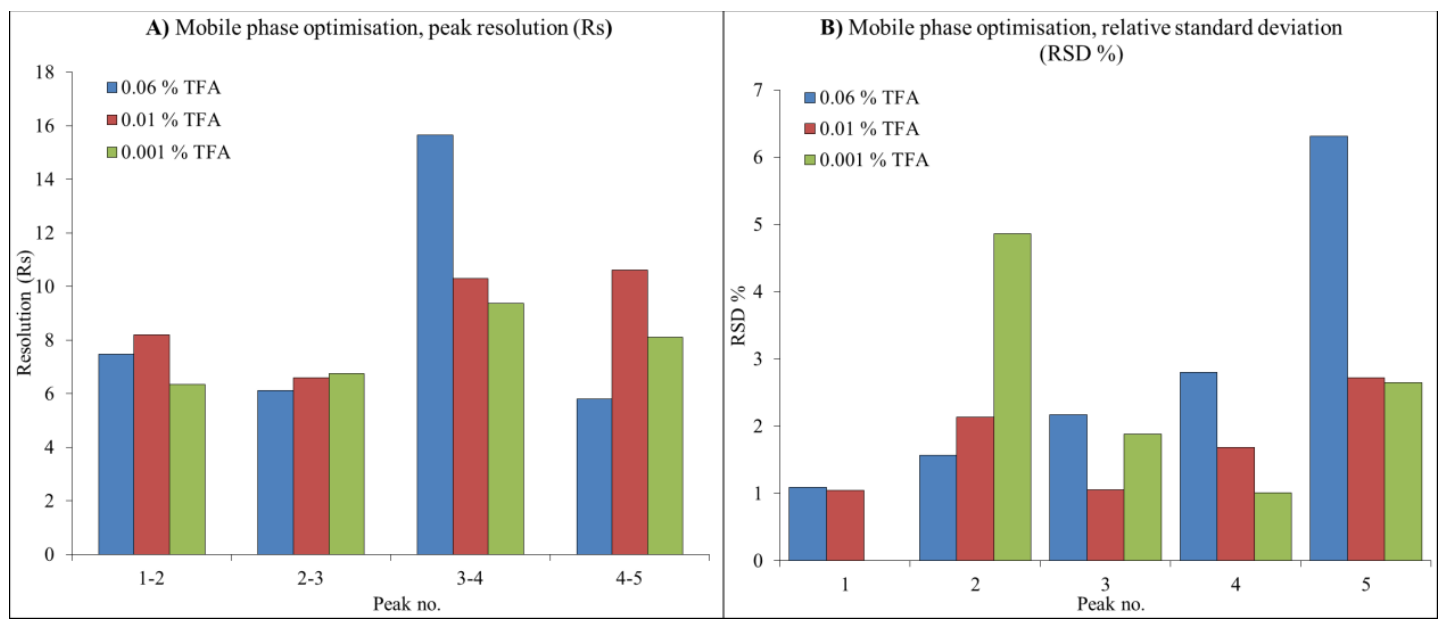

Figure 34: A) Resolution factor (Rs) between MAA peaks detected separated using the three different compositions of eluent A and B) Relative standard deviation (RSD \%) of the detected MAA peak areas 
for the three compositions of eluent A. Peak no: $1=$ shinorine; $\mathbf{2}=$ palythine; $3=$ asterina; $4=$ porphyra-334; 5 = palythinol.

Six different reverse phase (RP) columns were used to evaluate the resolution of peaks separated from crude extract 1 using varying column lengths and stationary phases; A) Alltima ${ }^{\circledR}$ Altech RP C18 column $(5 \mu \mathrm{m}, 4.6 \times 150 \mathrm{~mm})$; B) Synergi ${ }^{\mathrm{TM}}$ Fusion RP C18 column (4 $\mu \mathrm{m}, 4.6 \times 150 \mathrm{~mm}, 80 \AA$ A); C) Hichrom RP ACE excel super-C18 column ( $3 \mu \mathrm{m}, 4.6$ x 250 mm, $80 \AA$ A); D) Hichrom RP ACE excel C18- Amide column (3 $\mu \mathrm{m}, 4.6$ x 250 mm, $100 \AA$ ) , E) Hypercarb® RP C30 column $(5 \mu \mathrm{m}, 4.6$ x 100 mm,

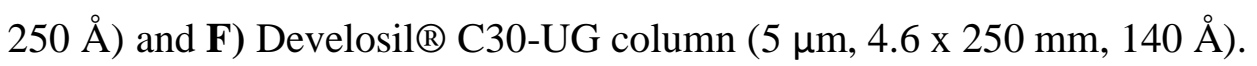

Only two columns (Figure 35A/2C) separated all peaks with good Rs (Table 20) and high repeatability, with RSD\% less than 5 for all peaks (Table 21). The best Rs was seen with ACE excel Super C18 column (C) with a length of $250 \mathrm{~mm}$ and a smaller particle size of $3 \mu \mathrm{m}$. Although the Rs is larger, the run time would need to be increased due to the final peak eluting at $25 \mathrm{~min}$ just before the equilibrating processes begins. Column A was therefore chosen as the Rs was still acceptable along with good repeatability and all peaks were eluted before the $25 \mathrm{~min}$.

Table 20: Resolution factor (Rs) between detected peaks separated using different RP-columns

\begin{tabular}{ccccccc}
\hline \multicolumn{7}{c}{ Resolution factor (Rs) } \\
\hline Peak & Column A & Column B & Column C & Column D & Column E & Column F \\
\hline $\mathbf{1 - 2}$ & 9.03 & 2.85 & 14.96 & 5.28 & 2.45 & 3.55 \\
$\mathbf{2 - 3}$ & 7.95 & 2.45 & 14.67 & 4.33 & - & 7.04 \\
$\mathbf{3 - 4}$ & 8.85 & 8.34 & 22.39 & 7.12 & - & 25.66 \\
$\mathbf{4 - 5}$ & 14.22 & - & 19.06 & - & - & 22.08 \\
$\mathbf{5 - 6}$ & 75.29 & - & 35.63 & - & - & - \\
$\mathbf{6 - 7}$ & 1.35 & - & 1.46 & - & - & - \\
\hline
\end{tabular}




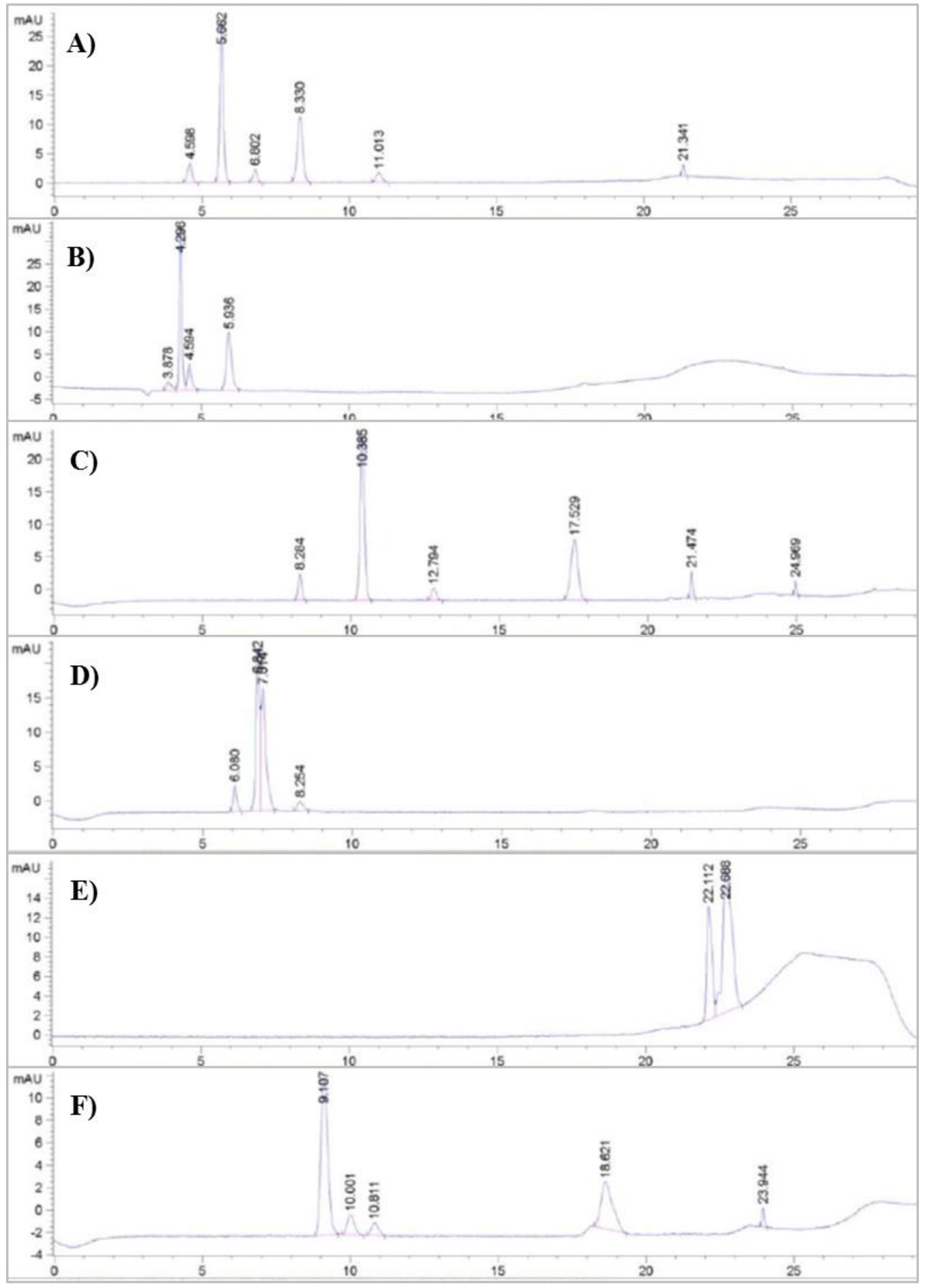

Figure 35: HPLC chromatograms of the MAA profile of crude extract 1 monitored at $330 \mathrm{~nm}$ using different columns: A) Alltima® Altech RP C18 column $(5 \mu \mathrm{m}, 4.6$ x $150 \mathrm{~mm})$; B) Synergi ${ }^{\mathrm{TM}}$ Fusion

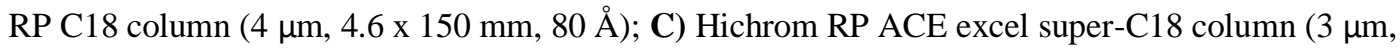

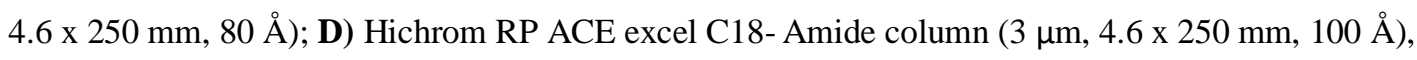
E) Hypercarb® RP C30 column $(5 \mu \mathrm{m}, 4.6$ x $100 \mathrm{~mm}, 250 \AA$ A $)$ and F) Develosil@ C30-UG column $(5$ $\mu \mathrm{m}, 4.6$ x $250 \mathrm{~mm}, 140 \AA$ ). 
Table 21: Retention time (RT), relative standard deviation (RSD \%) and UV-absorbance maxima ( $\lambda$ max) for each peak detected using different RP-columns. Non-detected peaks are represented by n.d.

\begin{tabular}{|c|c|c|c|c|c|c|c|c|c|c|c|c|c|}
\hline \multirow[b]{2}{*}{$\begin{array}{l}\text { Peak } \\
\text { no. }\end{array}$} & \multicolumn{2}{|c|}{ Column A } & \multicolumn{2}{|c|}{ Column B } & \multicolumn{2}{|c|}{ Column C } & \multicolumn{2}{|c|}{ Column D } & \multicolumn{2}{|c|}{ Column E } & \multicolumn{2}{|c|}{ Column F } & \multirow[b]{2}{*}{$\begin{array}{r}\lambda \max \\
(\mathbf{n m})\end{array}$} \\
\hline & $\begin{array}{c}\text { RT } \\
(\mathbf{m i n}) \\
\end{array}$ & $\begin{array}{c}\text { RSD } \\
\% \\
\end{array}$ & $\begin{array}{c}\text { RT } \\
(\mathrm{min}) \\
\end{array}$ & $\begin{array}{c}\text { RSD } \\
\% \\
\end{array}$ & $\begin{array}{c}\text { RT } \\
(\mathrm{min}) \\
\end{array}$ & $\begin{array}{c}\text { RSD } \\
\% \\
\end{array}$ & $\begin{array}{c}\text { RT } \\
(\mathrm{min})\end{array}$ & $\begin{array}{c}\text { RSD } \\
\% \\
\end{array}$ & $\begin{array}{c}\text { RT } \\
(\mathrm{min}) \\
\end{array}$ & $\begin{array}{c}\text { RSD } \\
\% \\
\end{array}$ & $\begin{array}{c}\text { RT } \\
(\mathbf{m i n}) \\
\end{array}$ & $\begin{array}{c}\text { RSD } \\
\% \\
\end{array}$ & \\
\hline 1 & 4.56 & 1.36 & 3.97 & 37.40 & 8.28 & 1.00 & 6.06 & 14.53 & 22.11 & 1.47 & 9.98 & 1.37 & 332 \\
\hline 2 & 5.65 & 1.84 & 4.31 & 15.12 & 10.40 & 0.95 & 6.83 & 32.75 & 22.68 & 1.76 & 9.09 & 1.18 & 320 \\
\hline 3 & 6.82 & 1.01 & 4.62 & 25.72 & 12.81 & 1.38 & 8.28 & 17.34 & n.d & - & 10.85 & 2.88 & 330 \\
\hline 4 & 8.34 & 0.68 & 6.00 & 30.68 & 17.60 & 0.39 & 6.98 & 15.63 & n.d & - & 18.69 & 2.65 & 332 \\
\hline 5 & 11.06 & 1.47 & n.d & - & 21.46 & 1.42 & n.d & - & n.d & - & n.d & - & 330 \\
\hline 6 & 21.34 & 3.30 & 18.20 & 13.86 & 24.96 & 2.01 & n.d & - & n.d & - & 23.95 & 2.12 & 356 \\
\hline 7 & 21.43 & 1.71 & n.d & - & 25.05 & 1.40 & n.d & - & n.d & - & n.d & - & 360 \\
\hline
\end{tabular}




\subsubsection{Method validation}

\section{Crude extracts 1, 2 and 3}

Following the optimisation of the protocol, all crude extracts were analysed to ensure good separation and repeatability with the chosen concentration of TFA and column type (Figure 36).

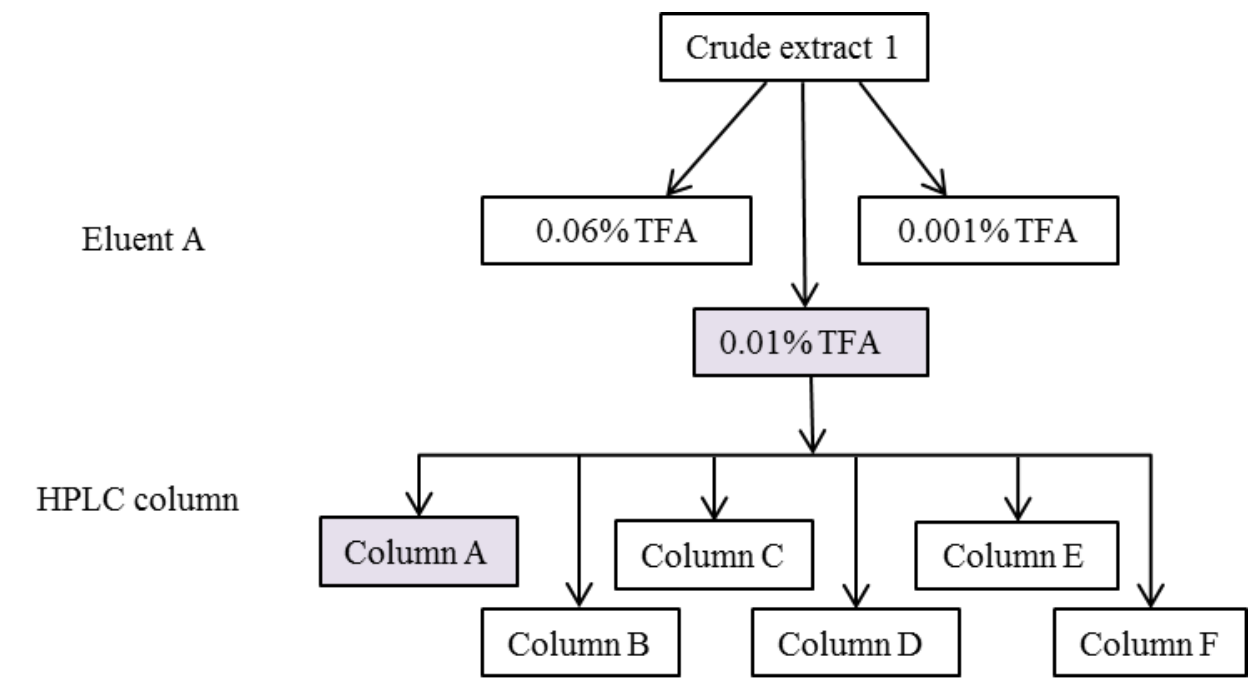

Figure 36: Optimisation protocol showing the different concentrations of TFA in eluent A followed by the different columns used.

Firstly, all three extracts were analysed using UV-visible spectroscopy (Figure 37) which showed the similar $\lambda \max$ of crude extract 1 and 2 with the characteristic usujirene shoulder seen in crude extract 1 only. Crude extract 3 is shifted to $\lambda$ max of $334 \mathrm{~nm}$ as it contains shinorine and porphyra-334 only.

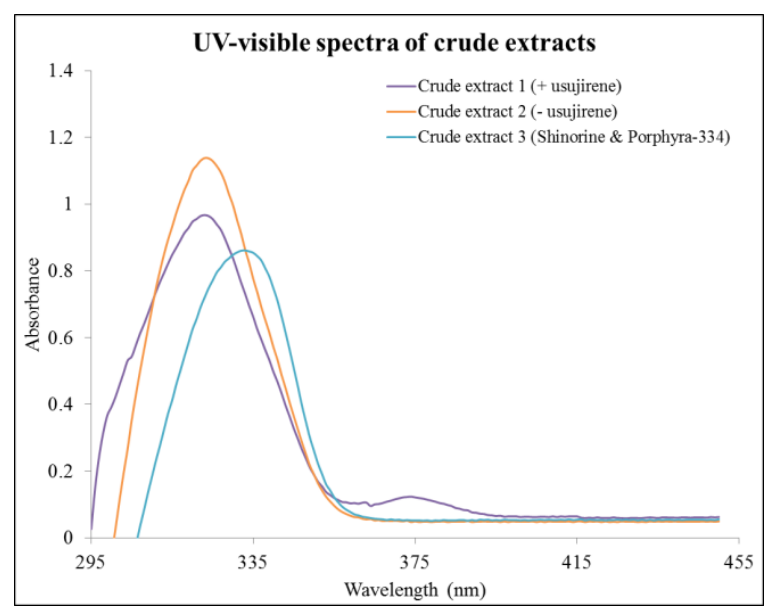

Figure 37: UV-visible spectra $(295-455 \mathrm{~nm})$ of crude extract 1 (+ usujirene), crude extract 2 (usujirene) and crude extract 3 (Shinorine \& Porphyra-334). 
Good separation was seen with all crude extracts (Figure 38) with RSD\% ( $n=3)$ less than 2.5 in all samples (Table 22) when using the optimised method.

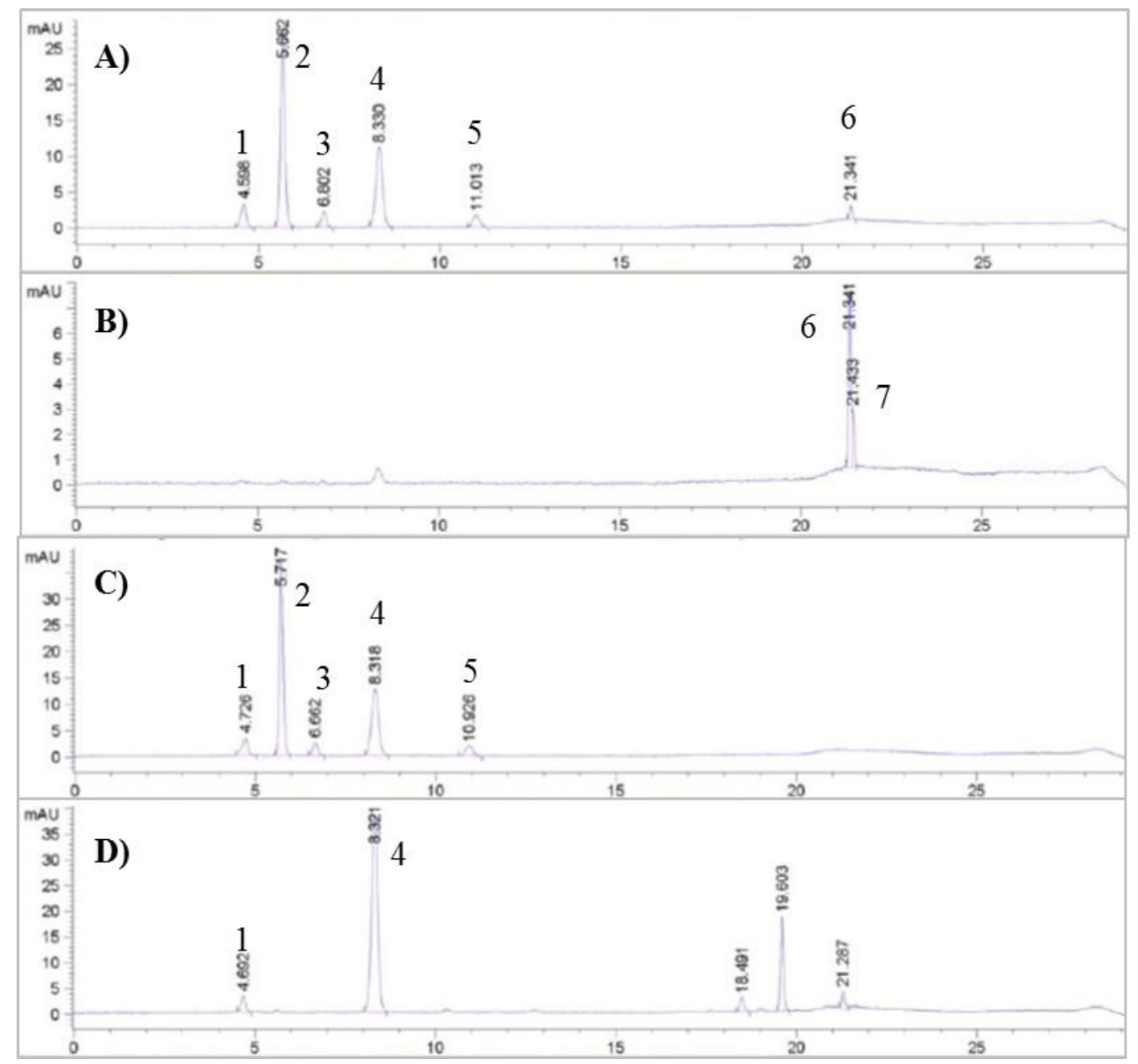

Figure 38: HPLC chromatograms of the MAA profiles of; A) crude extract 1 at $330 \mathrm{~nm}$; B) crude extract 1 at $360 \mathrm{~nm}$; C) crude extract 2 at $330 \mathrm{~nm}$ and D) crude extract 3 at $330 \mathrm{~nm}$. 
Table 22: Peak identifications including retention time (RT), relative standard deviation (RSD \%), resolution factor (Rs) between peaks and UV-absorbance maxima $(\lambda \max )$.

\begin{tabular}{|c|c|c|c|c|c|}
\hline \multicolumn{6}{|c|}{ Crude extract 1 (+ usujirene) } \\
\hline Peak no. & MAA & RT (min) & RSD \% & Rs (between peaks) & $\lambda \max (\mathbf{n m})$ \\
\hline 1 & Shinorine & 4.56 & 1.36 & - & 332 \\
\hline 2 & Palythine & 5.65 & 1.84 & $9.03(1 / 2)$ & 320 \\
\hline 3 & Asterina & 6.82 & 1.01 & $7.95(2 / 3)$ & 330 \\
\hline 4 & Porphyra-334 & 8.34 & 0.68 & $8.85(3 / 4)$ & 332 \\
\hline 5 & Palythinol & 11.06 & 1.47 & $14.22(4 / 5)$ & 330 \\
\hline 6 & Usujirene & 21.34 & 3.3 & $75.29(5 / 6)$ & 356 \\
\hline 7 & Palythene & 21.43 & 1.71 & $1.35(6 / 7)$ & 360 \\
\hline \multicolumn{6}{|c|}{ Crude extract 2 (- usujirene) } \\
\hline Peak no. & MAA & RT (min) & RSD \% & Rs (between peaks) & $\lambda \max (\mathbf{n m})$ \\
\hline 1 & Shinorine & 4.62 & 2.16 & - & 332 \\
\hline 2 & Palythine & 5.69 & 1.47 & $9.22(1 / 2)$ & 320 \\
\hline 3 & Asterina & 6.73 & 1.07 & $7.44(2 / 3)$ & 330 \\
\hline 4 & Porphyra-334 & 8.24 & 0.92 & $8.76(3 / 4)$ & 332 \\
\hline 5 & Palythinol & 10.97 & 0.41 & $13.76(4 / 5)$ & 330 \\
\hline 6 & Usujirene & n.d & - & - & - \\
\hline 7 & Palythene & n.d & - & - & - \\
\hline \multicolumn{6}{|c|}{ Crude extract 3 (Shinorine \& Porphyra-334) } \\
\hline Peak no. & MAA & RT (min) & RSD \% & Rs (between peaks) & $\lambda \max (\mathbf{n m})$ \\
\hline 1 & Shinorine & 4.62 & 2.1 & - & 332 \\
\hline 2 & Palythine & n.d & - & - & - \\
\hline 3 & Asterina & n.d & - & - & - \\
\hline 4 & Porphyra-334 & 8.21 & 1.22 & $24.84(1 / 4)$ & 332 \\
\hline 5 & Palythinol & n.d & - & - & - \\
\hline 6 & Usujerine & n.d & - & - & - \\
\hline 7 & Palythene & n.d & - & - & - \\
\hline
\end{tabular}




\subsubsection{P. palmata crude extracts}

P. palmata was collected from the Welsh coast (Langland) during summer 2017 to extract and use in method validation of the optimised HPLC analysis to use as an unknown sample. All MAAs were extracted and eluted with good separation (Figure 39).

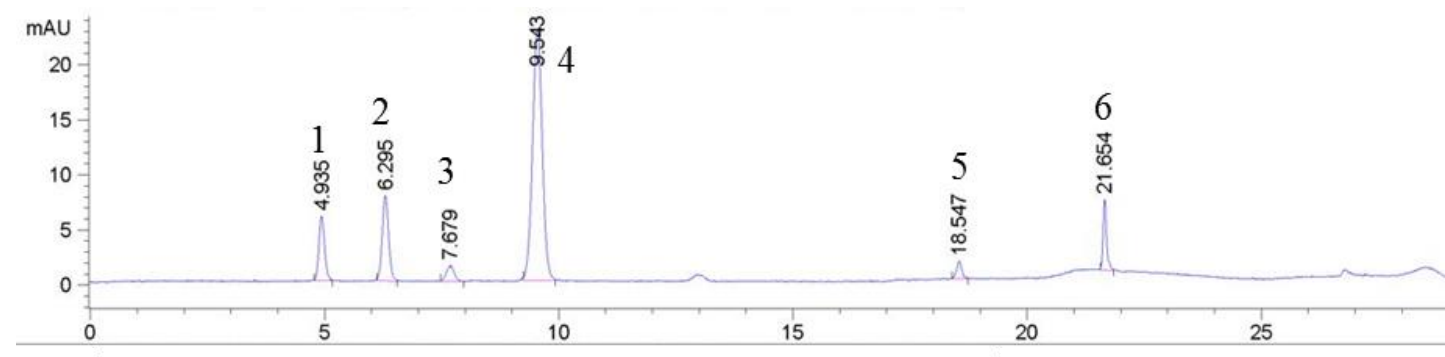

Figure 39: HPLC chromatograms of the MAA profile of the crude $P P$ extract showing absorbance at $330 \mathrm{~nm}$ (top) and $360 \mathrm{~nm}$ (bottom): 1) Shinorine, $\lambda \max =332 \mathrm{~nm}$; 2) Palythine, $\lambda \max =320 ; 3$ ) Asterina, $\lambda \max =332 \mathrm{~nm}$; 4) Porphyra-334, $\lambda \max =334 ; 5)$ Usujirene, $\lambda \max =356 \mathrm{~nm}$ and 6) Palythene, $\lambda \max$ $=358 \mathrm{~nm}$.

The validated method was used in subsequent analyses of MAA production in $C$. fritschii during UV exposure. 


\section{Time-series analysis of $C$. fritschii during UV exposure}

\subsubsection{Intracellular and extracellular analysis of $C$. fritschii during UV-B stress}

The metabolite profiles of $C$. fritschii cultures $(\mathrm{n}=3)$ were investigated during $48 \mathrm{~h}$ of UV-B exposure. At each time point $(0,2,6,12,24$ and 48 h) intracellular and extracellular metabolites were analysed by untargeted GC-MS to evaluate the global changes in metabolite production during UV-B stress. Dry weight, carotenoid and MAA analysis was also conducted at each time-point.

A reduction in average dry weight was measured over $24 \mathrm{~h}$ of UV-B exposure with recovery of the initial biomass concentration and further growth measured between 24 and 48 h (Figure 40Error! Reference source not found.).

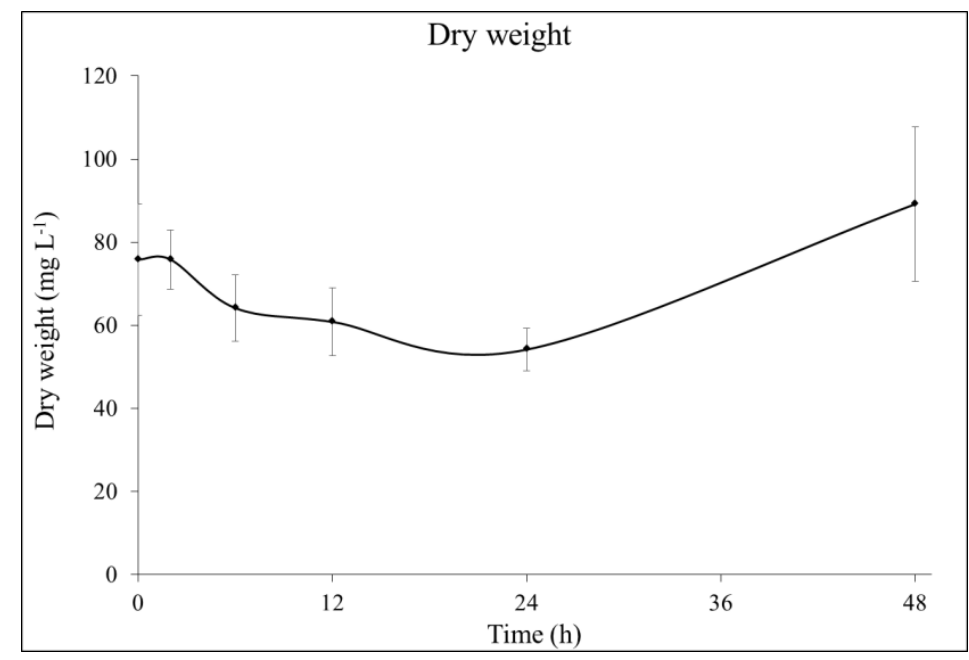

Figure 40: Dry weight measurements of $C$. fritschii cultures during $48 \mathrm{~h}$ of supplemented UV-B exposure. All values are the mean of three biological replicates \pm SE.

Carotenoid concentration (Figure 41A) and MAA content (Figure 41B) were analysed by UV-visible spectroscopy and high performance liquid chromatography (HPLC) respectively. Total carotenoid concentration decreased after $2 \mathrm{~h}(p<0.05)$; with a steady significant increase up to $48 \mathrm{~h}$ with a final concentration of $2.59 \mu \mathrm{g} / \mathrm{mg}$ dry weight $(p<0.05)$.

As described above, UV-B also induces the production of the photoprotective compounds, MAAs. The two forms found in C. fritschii are mycosporine-glycine (mgly) and shinorine [20], both were detected during this experiment with peaks identified using their retention time and absorption maxima ( $\lambda \max )$ values. As expected, an increase in shinorine (retention time $\sim 4.9 \min , \lambda \max =334 \mathrm{~nm}$ ) was 
observed with increasing length of UV-B exposure. No significance was observed with m-gly (retention time $\sim 10.8 \mathrm{~min}, \lambda \max =310 \mathrm{~nm}$ ) content during this experimental time series.

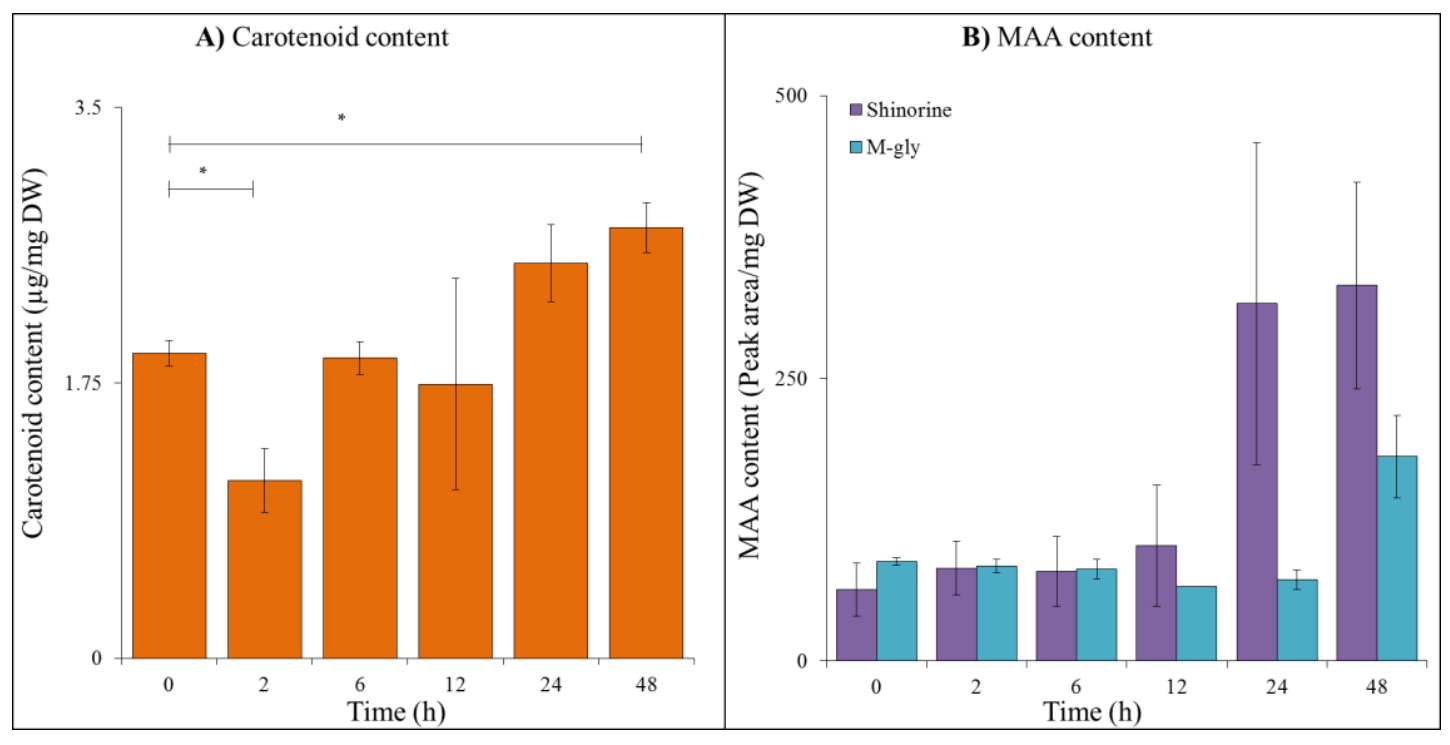

Figure 41: Carotenoid and mycosporine-like amino acid (MAA) analysis of $C$. fritschii extracts during UV-B exposure. A) Total carotenoid concentration as measured by UV-visible spectroscopy and B) shinorine and mycosporine-glycine (m-gly) content measured by HPLC analysis. All values are the mean of three biological replicates (normalised to dry weight) \pm standard error. Statistical significance was measured using a two sample T-test with equal variance; $*=0.05>p \geq 0.01, * *=0.01>p \geq 0.001$ and $* * *=p<0.001$.

A total of 300 and 412 peaks were detected from the intracellular and extracellular time-series data respectively. Using a match factor of $60 \%$ or above, 135 and 218 peaks were putatively identified within the intracellular and extracellular data respectively. The identified chemical structures belonged to a variety of classes such as; acids, alcohols, amino acids, aromatics, fatty acids, heterocycles and sugars. 

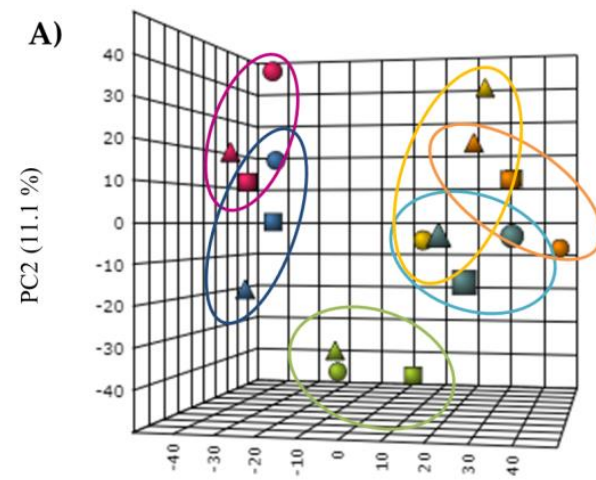

PC1 $(17 \%)$

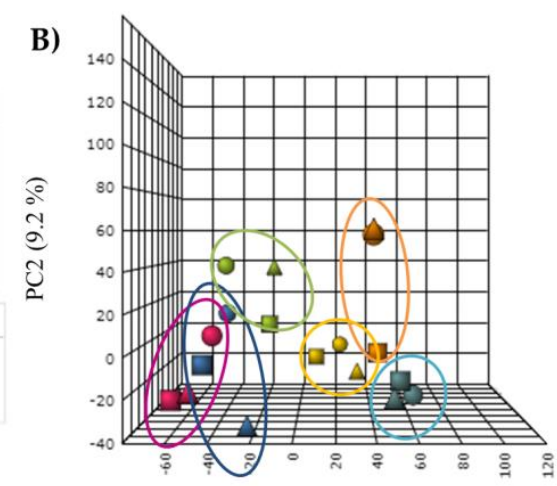

PC1 $(18.9 \%)$

Figure 42: Principle component analysis (PCA) of A) intracellular and B) extracellular GC-MS data of UV-B exposed (PAR + UV-B) C. fritschii cultures showing PC1 vs PC2 only. Each ring represents distribution of biological replicates.

A Principle Component Analysis (PCA) model was used as an unsupervised multivariate statistical tool to plot and visualise the variance between UV-B exposed samples over time. A total variance of $37.4 \%$ for intracellular (Figure 42A, PC1 = $17.4 \%, \mathrm{PC} 2=10.9 \%, \mathrm{PC} 3=9.1 \%)$ and $36.5 \%$ for extracellular (Figure 42B, PC1 = $18.9 \%, \mathrm{PC} 2=9.3 \%, \mathrm{PC} 3=8.3 \%)$ was observed.

The results of the PCA for intracellular samples (Figure 42A) showed good separation over time between the control $(0 \mathrm{~h})$ and $6,12,24$ and $48 \mathrm{~h}$ of UV-B. Less variation was observed between 0 and $2 \mathrm{~h}$ of UV-B with clustering seen between 12, 24 and 48 $\mathrm{h}$ of UV-B. This result was consistent with the two-sample t-Test results comparing control $(0 \mathrm{~h})$ with each time point where the number of significant features reducing in levels increases with length of UV-B exposure. After a one-way analysis of variance (ANOVA) with repeated measures, 109 peaks with overall significant differences within the time-series were observed with $p<0.05$ (Figure 43A), 10 of which remained significant after BF correction.

From the extracellular data PCA (Figure 42B) a similar pattern was observed with increasing variance with increasing length of UV-B exposure. Statistically significant changes between control $(0 \mathrm{~h})$ and each time point, measured using a two-sample tTest also showed increasing significance $(p<0.05)$ with increasing length of UV-B up to $24 \mathrm{~h}$. A one-way ANOVA with repeated measures calculated 117 statistically significant peaks with $p<0.05$ (Figure 43A). 

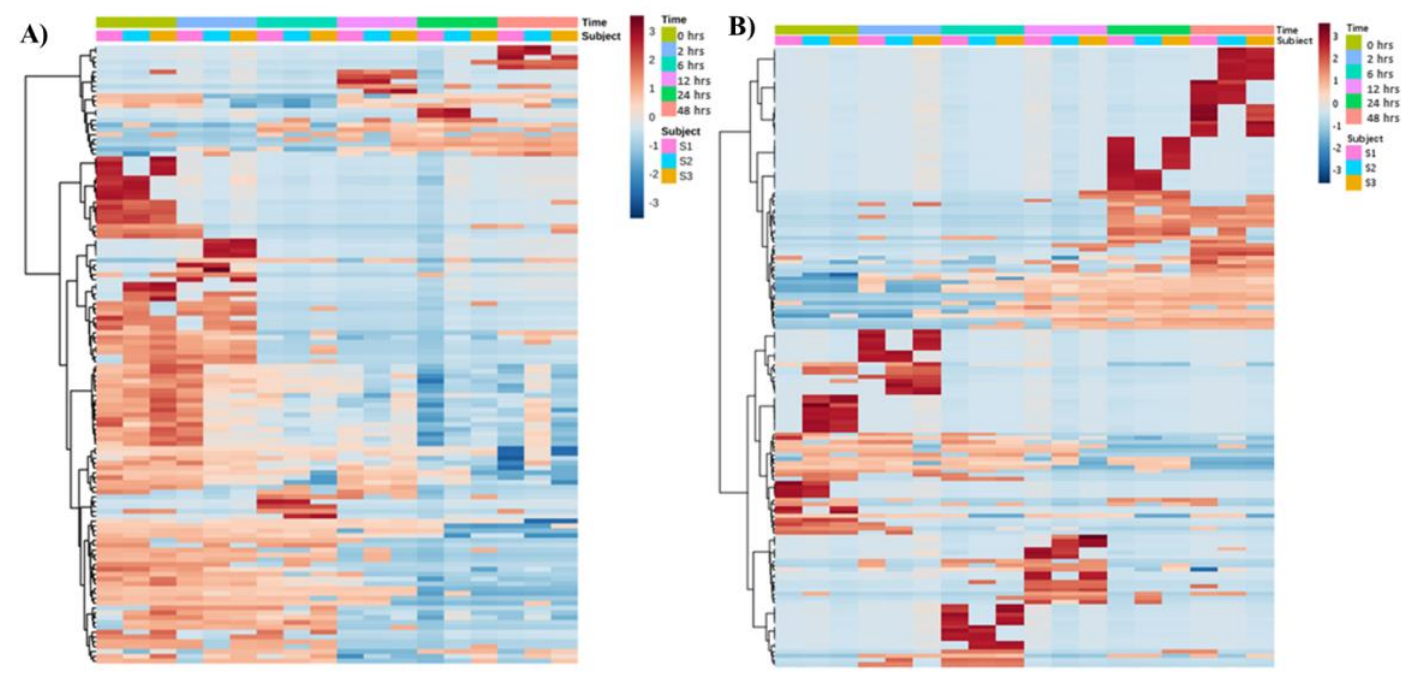

Figure 43: Hierarchical heatmap visualisation of the significant A) intracellular and B) extracellular peak intensities $(p<0.05)$ during UV-B exposure using a one-way repeated measure ANOVA in MetaboAnalyst. Data is arranged in triplicate with increasing length of UV-B exposure from left to right $(0-48 \mathrm{~h})$.

\subsubsection{Intracellular metabolites}

28 metabolites (13 represented for simplicity Figure 44), selected as being involved in the central carbon and nitrogen metabolism within cyanobacteria, were identified within the intracellular GC-MS results (Table S5). Many changes in metabolite levels were observed comparing between time points. Glucose, pyruvate and lactate all decreased in abundance after UV-B exposure with significant reduction after $2 \mathrm{~h}$ (pyruvate $p<0.05,0$ vs. $2 \mathrm{~h}$ ), $6 \mathrm{~h}$ (glucose $p<0.05,2$ vs. $6 \mathrm{~h}$ ) and $12 \mathrm{~h}$ (lactate $p<0.05$, 0 vs. $12 \mathrm{~h}$ ). Lactate was present during the whole time-course whereas glucose and pyruvate were not detected after $6 \mathrm{~h}(p<0.05)$ and $24 \mathrm{~h}(p<0.001)$ respectively.

6 proteinogenic amino acids were detected; ser, gly, glu, pro, tyr and phe. All detected amino acids decreased after 6 or $12 \mathrm{~h}$ of exposure with the exception of pro. A decrease in tyr, phe and gly was seen after $6 \mathrm{~h}$ (tyr $p<0.05,0$ vs. $6 \mathrm{~h}$; phe $p<0.01,2 \mathrm{vs.} 6 \mathrm{~h}$; gly $p<0.05,2$ vs. $6 \mathrm{~h}$ ) of UV-B followed by no detection at $6,12,24$, and $48 \mathrm{~h}$ (tyr $p<0.05$; phe $p<0.01$; gly $p<0.05)$. Ser and glu decreased significantly after $12 \mathrm{~h}$ of treatment ( $\operatorname{ser} p<0.05$, 0 vs. $12 \mathrm{~h}$; glu $p<0.05,0$ vs. $12 \mathrm{~h}$ ), ser was not detected between 12 and $48 \mathrm{~h}(p<0.05)$ whereas glu was detected throughout the time series. Proline showed no significant decrease after UV-B exposure with a significant increase observed after 24 h $(p<0.05,6$ vs. 24 h). 
The fatty acids stearic acid, palmitic acid and mystiric acid all decreased significantly after $2 \mathrm{~h}$ of treatment $(p<0.05)$ and their abundance remained lowered throughout the time series (stearic acid $p<0.01,0$ vs. 48 h; palmitic acid $p<0.05,0$ vs. 48 h; mystitric acid $p<0.01,0$ vs. $48 \mathrm{~h}$ ). 


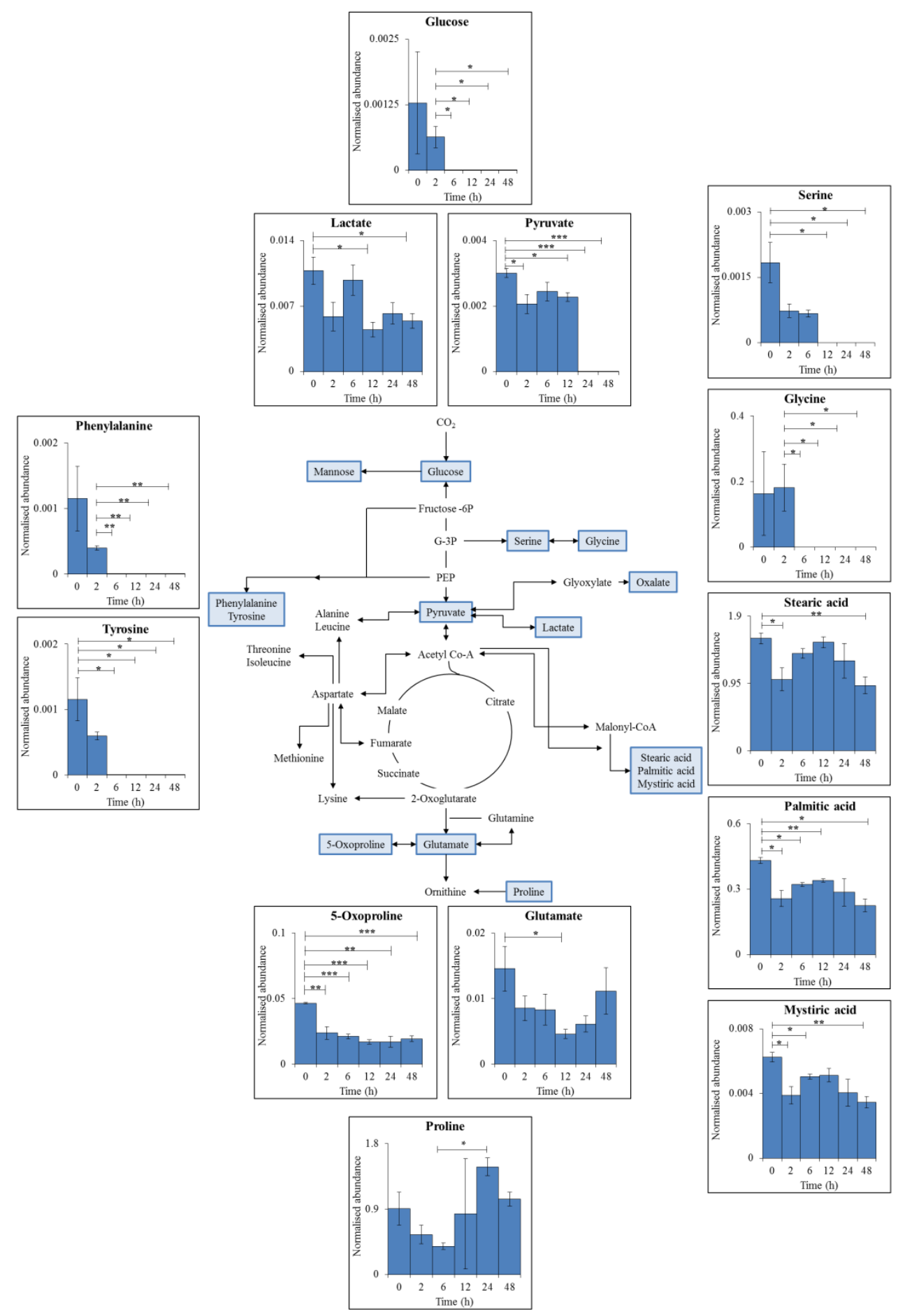

Figure 44: Schematic representation of a generalised reduced carbon metabolism in the cyanobacterium C. fritschii showing glycolysis, the TCA cycle, amino acid and fatty acid biosynthesis. Primary metabolites identified in intracellular samples using GC-MS are highlighted in blue with each insert presenting mean values of normalised abundance (normalised to internal standard and dry weight) $\pm \mathrm{SE}$ of each metabolite during supplemented UV-B exposure (PAR + UV-B). Statistical significance between control $(0 \mathrm{~h})$ and UV-B exposure $(2,6,12,24$ and $48 \mathrm{~h})$ and between each treatment time point was measured using a two sample $\mathrm{t}$-Test with equal variance; $*=0.05>p \geq 0.01, * *=0.01>p \geq 0.001$ and $* * *=p<0.001$. 


\subsubsection{Extracellular metabolites}

29 biologically relevant dissolved metabolites (Table S5) were detected within the extracellular data set (13 represented for simplicity, Figure 45). Citrate, a component of BG-11 medium [5] and involved in TCA cycle, was consistently detected throughout the time series (ANOVA, $p<0.05$ ) along with succinate. Other TCA substrates; malate and fumarate were also detected at $0 \mathrm{~h}$ with decreasing abundance after $2 \mathrm{~h}$ of UV-B (malate, $p<0.001$ ). Other metabolites detected at $0 \mathrm{~h}$ which decreased after UV-B exposure were leucine ( $p<0.01,0$ vs. $6 \mathrm{~h}$ ), putrescine $(p<0.05$, 0 vs. 2 h) octanoic acid ( $p<0.001,0$ vs. 24 h) mystiric acid ( $p<0.01,2$ vs. 24 h) and fructose ( $p<0.01,0$ vs. 2 h). Accumulation of the sugars galactose, xylose, lyxose and arabinose was seen after $6 \mathrm{~h}$ (galactose $p<0.01$; arabinose $p<0.01$ ) $12 \mathrm{~h}$ (arabinose $p<0.05$ ), $24 \mathrm{~h}$ (arabinose $p<0.05$; xylose $p<0.05$; lyxose $p<0.01$ ) and $48 \mathrm{~h}$ (galactose $p<0.05$; arabinose $p<0.001$; lyxose $p<0.01)$ of UV-B exposure. Trehalose was also identified throughout the time series with no significant changes. 

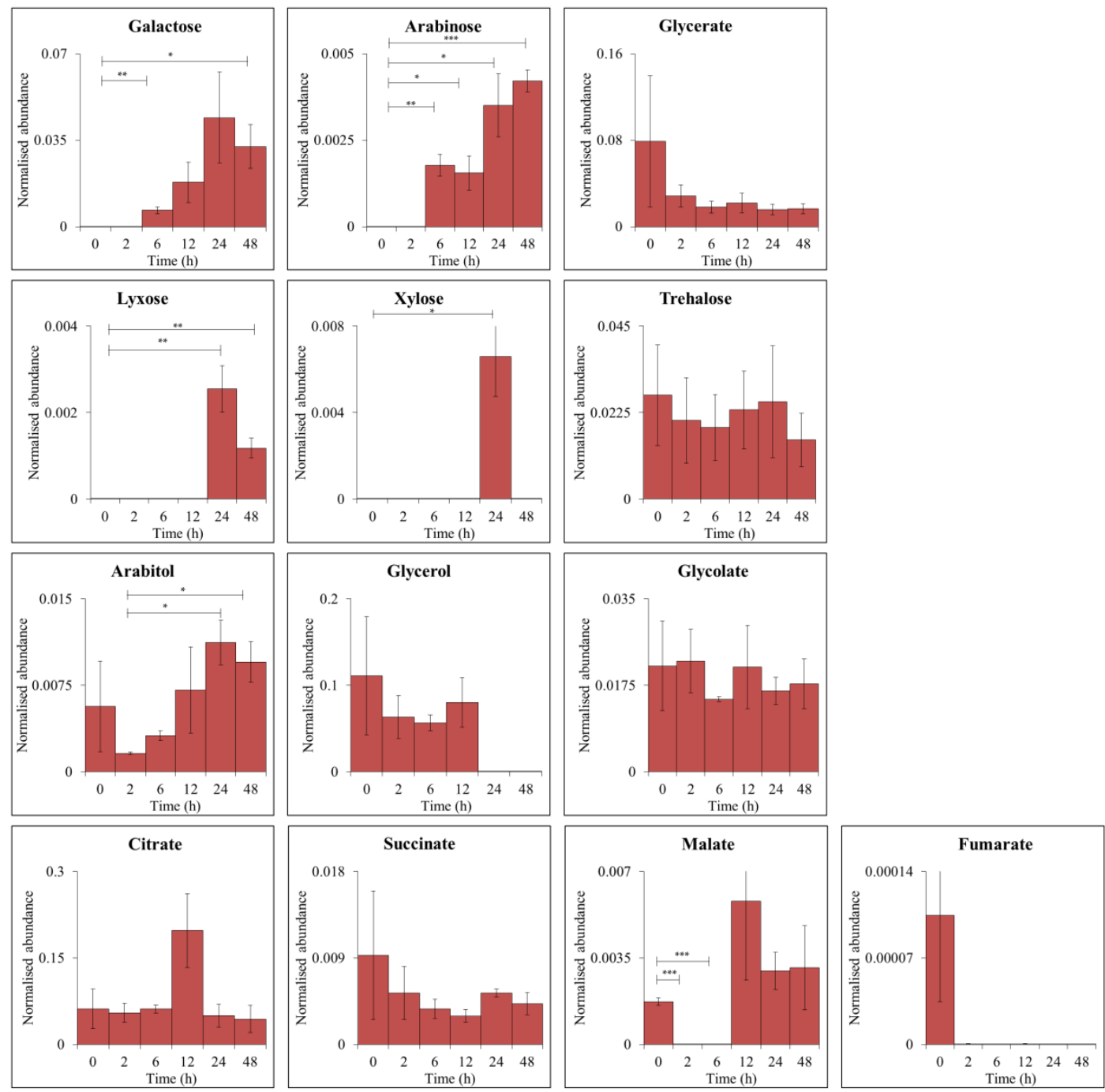

Figure 45: Time-series extracellular GC-MS data of $C$. fritschii over $48 \mathrm{~h}$ of UV-B exposure showing primary metabolites found in extracellular samples only. Significant difference in metabolite levels were measured using a two-sample t-Test comparing control $(0 \mathrm{~h})$ and UV-B exposure $(2,6,12,24$ and 48 h) and between each treatment time point, $*=0.05>p \geq 0.01, * *=0.01>p \geq 0.001$ and $* * *=p<0.001$.

7 metabolites from the identified biologically relevant pool were found in both intraand extracellular metabolite samples (Figure 46). The fatty acid mystiric acid shows a similar pattern of reduced abundance with increasing length of UV-B in both samples (intracellular 0 vs. 48 h, $p<0.05$; extracellular 2 vs. 24 h, $p<0.05$ ). Ethanolamine and 2-oxobutanoate show opposite patterns with intracellular levels decreasing ( 0 vs. $48 \mathrm{~h}$, $p<0.05$ ) and extracellular levels increasing (ethanolamine 0 vs. $24 \mathrm{~h}, p<0.01,2$ oxobutanoate 0 vs. 24 h, $p<0.001$ ). 
A)

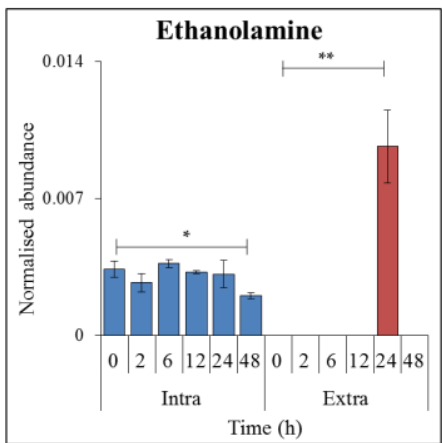

B)
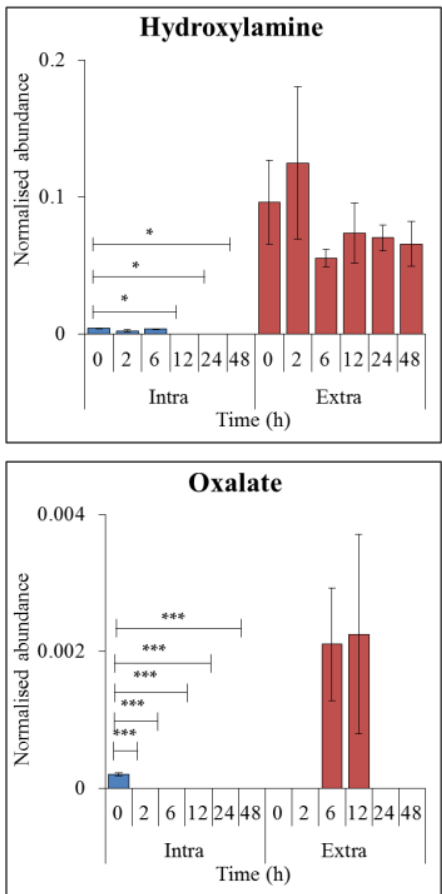
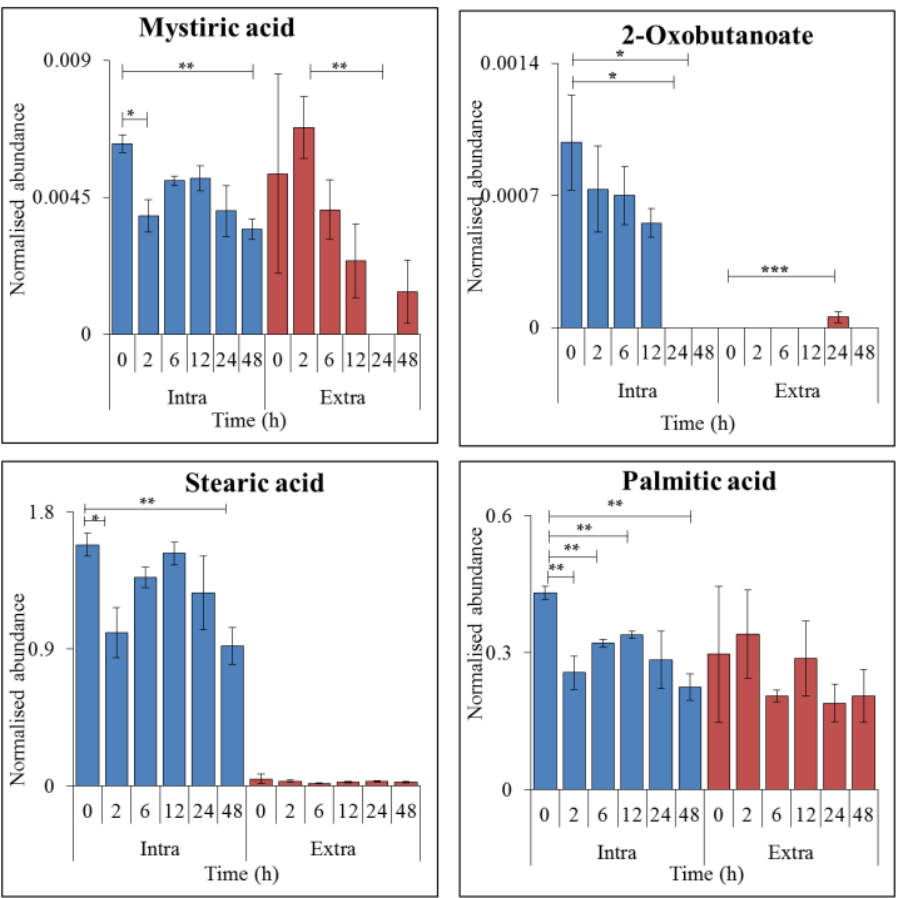

Figure 46: Time-series GC-MS data of $C$. fritschii during 48 h of UV-B exposure showing primary metabolites found in both intra- and extracellular samples. A) Metabolites showing significantly different changes $(p<0.05)$ over time in both intra- and extracellular data; B) metabolites showing significantly different changes $(p<0.05)$ over time in intracellular samples only. Significant differences were measured using a two-sample t-Test comparing control $(0 \mathrm{~h})$ to each treatment time point $(2,6$, 12,24 and $48 \mathrm{~h}$ ) and between treatment time points, $*=0.05>p \geq 0.01, * *=0.01>p \geq 0.001$ and $* * *=$ $p<0.001$. 


\subsubsection{Intracellular analysis of $C$. fritschii during UV-A stress response}

C. fritschii cultures were exposed to supplemented UV-A for $48 \mathrm{~h}$. The dry weight measured decreased significantly to an average of $54.2 \mathrm{mg} \mathrm{L}^{-1}$ after $6 \mathrm{~h}$ of supplemented UV-A exposure ( $p<0.01,0$ vs $6 \mathrm{~h}$ ) with an increase up to an average of $62.5 \mathrm{mg} \mathrm{L}^{-1}$ after $48 \mathrm{~h}$ with no significance measured (Figure 47A). Total carotenoid content (Figure 47B) decreased after $2 \mathrm{~h}$ of UV-A exposure ( $p>0.05$, no significance) with a statistically significant increase after $12 \mathrm{~h} \mathrm{(2} \mathrm{vs.} 12 \mathrm{~h}, p<0.05)$ and $48 \mathrm{~h}(2 \mathrm{vs}$. $48 \mathrm{~h}, p<0.05)$.

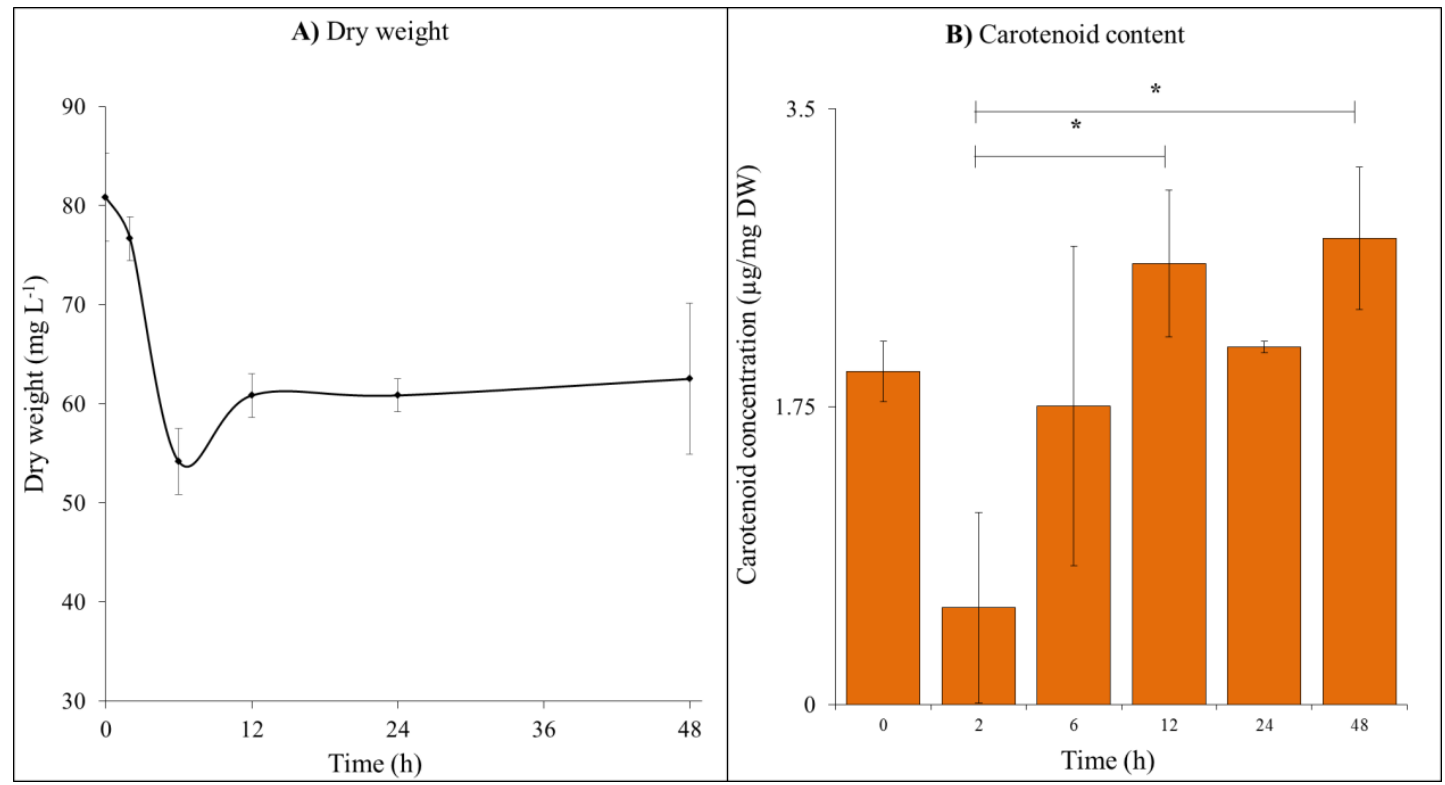

Figure 47: A) Dry weight $\left(\mathrm{mg} \mathrm{L}^{-1}\right)$ and B) carotenoid content of $C$. fritschii cultures $(\mathrm{n}=3)$ during supplemented UV-A exposure over $48 \mathrm{~h}$. Statistical significance was calculated comparing control $(0$ h) and each time point $(2,6,12,24$ and $48 \mathrm{~h})$ using a two-sample t-Test, $*=0.05>p \geq 0.01, * *=$ $0.01>p \geq 0.001$ and $* * *=p<0.001$.

Intracellular metabolite levels within $C$. fritschii were investigated during $48 \mathrm{~h}$ of UVA exposure using GC-MS analysis. After data processing the peaks were aligned in SpectConnect to yield a total of 210 detected peaks throughout the 48-h time-series. Of the 210 peaks detected, 175 were classified as 'true-hits' with 91 putatively identified (level 2; identified due to similarity to online spectra [63]) and 84 remaining unassigned (level 4; unknown [63]) using a match factor of $\geq 60 \%$ comparing to online spectra 
Comparing control $(0 \mathrm{~h})$ and each time point using a two sample t-Test, $24 \mathrm{~h}$ of UV-A had the highest significant difference in peak levels with 115 peaks (out of 175), of those 84 increased in levels (positive $\log 2 \mathrm{FC}$ ) and 31 reduced in levels (negative $\log 2 \mathrm{FC}$, (Table 23). $6 \mathrm{~h}$ of UV-A had the highest percentage (78\%) of significantly different peaks with elevated levels (81 with a positive $\log 2 \mathrm{FC}$ ). After $48 \mathrm{~h}$ of UV-A exposure a larger percentage (57\%) of significantly different peaks showed a reduction in normalised abundance ( 34 with a negative $\log 2 \mathrm{FC}$ ).

Table 23: Comparison of statistically significant $(p<0.05)$ peaks (out of 175) and number of those significant peaks with a positive or negative $\log 2 \mathrm{FC}$. A positive $\log 2 \mathrm{FC}$ indicates an increase in metabolite level and a negative $\log 2 \mathrm{FC}$ value indicates a decrease in metabolite level. Both significance and $\log 2 \mathrm{FC}$ were calculated comparing control $(0 \mathrm{~h})$ and 2, 6, 12, 24 and $48 \mathrm{~h}$ of UV-A exposure.

\begin{tabular}{cccc}
\hline $\begin{array}{c}\text { UV-A exposure } \\
\text { (h) }\end{array}$ & $\begin{array}{c}\text { No. of peaks, } \\
\boldsymbol{p}<\mathbf{0 . 0 5}^{\mathbf{a}}\end{array}$ & $\begin{array}{c}\text { No. of peaks, } \\
\text { positive log2FC }\end{array}$ & $\begin{array}{c}\text { No. of peaks, } \\
\text { negative log2FC }\end{array}$ \\
\hline 0 vs. $\mathbf{2}$ & 49 & 28 & 21 \\
0 vs. 6 & 104 & 81 & 23 \\
0 vs. $\mathbf{1 2}$ & 100 & 70 & 30 \\
0 vs. $\mathbf{2 4}$ & 115 & 84 & 31 \\
0 vs. $\mathbf{4 8}$ & 60 & 26 & 34 \\
\hline
\end{tabular}

${ }^{a}=$ Significanct differences wascalculated using a two sample $\mathrm{t}$-Test with equal variance comparing control $(0 \mathrm{~h})$ to each UV-A exposed time point.

Twenty-nine metabolites (10 shown for simplicity in, Figure 48) were identified throughout the time-series that have roles as primary metabolites within cyanobacterial metabolism including; glycolysis, the TCA cycle, amino acid and fatty acid biosynthesis (Table S6). The four amino acids; glu, leu, ser and phe were identified with a match factor of $\geq 60 \%$. A significant reduction in leu and phe levels was seen after $6 \mathrm{~h}(p<0.01$ and $p<0.001$ respectively) of supplemented UV-A exposure (UV-A + PAR), with glu decreasing significantly after $24 \mathrm{~h}(p<0.05)$. Ser levels increased significanty after $2 \mathrm{~h}$ of UV-A $(p<0.01)$.

Four saturated fatty acids were also putatively identified, 3 of which showed significant increases after $2 \mathrm{~h}$ (eicosanoic acid, $p<0.05$ ), $12 \mathrm{~h}$ (stearic acid, $p<0.05$ ) and 
$24 \mathrm{~h}$ (palmitic acid, $p<0.05$ ) of UV-A exposure with mystiric acid levels showing steady levels with no significant changes $(p>0.05)$.

The sugars mannose and sucrose were identified with significance during UV-A exposure, with sucrose levels decreasing significantly (0 vs. $24 \mathrm{~h}, p<0.05)$ and mannose increasing significantly ( 0 vs. 2 h, $p<0.01)$ during the time-series.

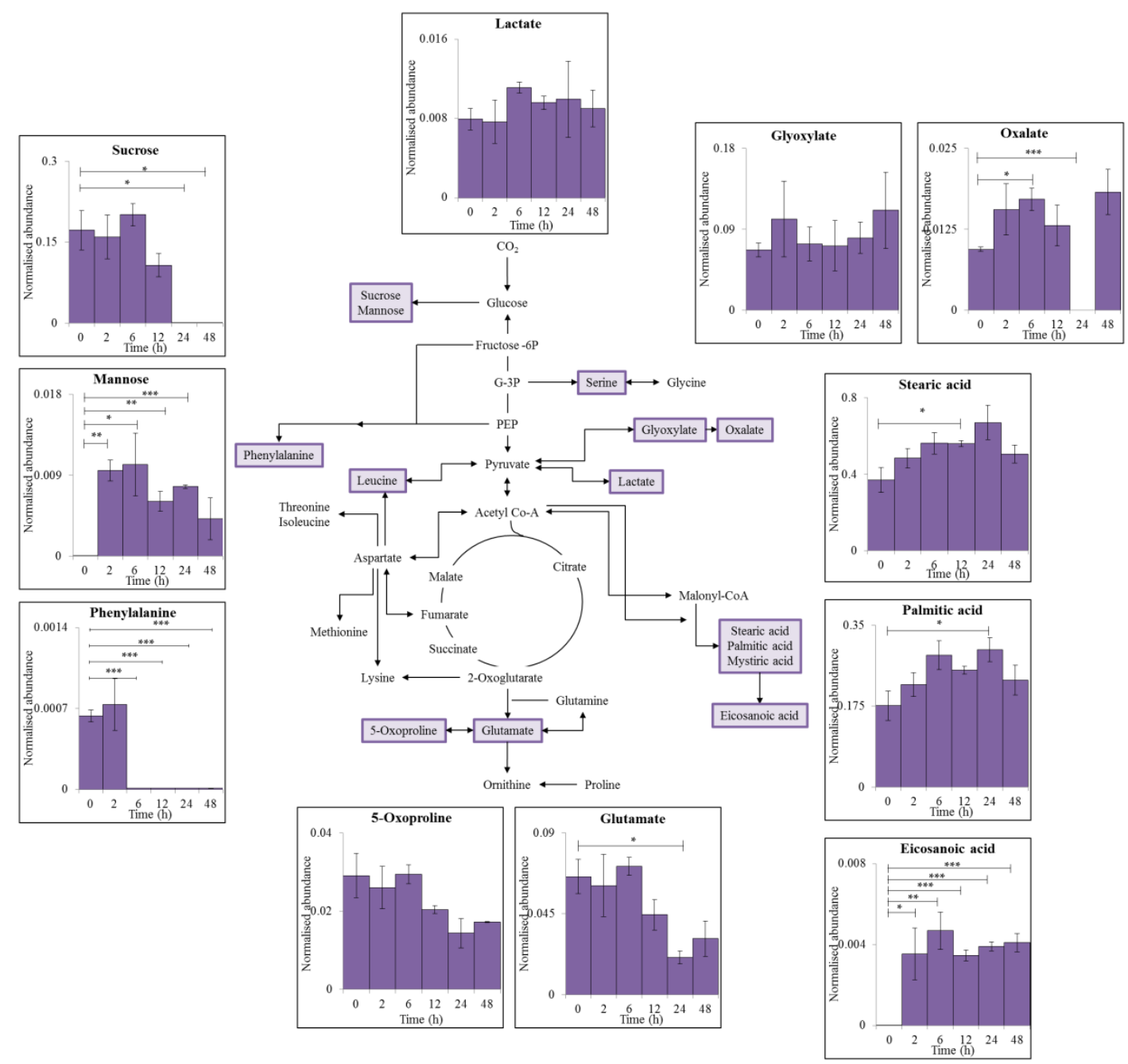

Figure 48: Schematic representation of a generalised reduced carbon metabolism in the cyanobacterium C. fritschii. Ten primary metabolites were identified in intracellular samples using GC-MS and are highlighted in purple with each graph presenting mean values of normalised abundance (normalised to internal standard and dry weight) \pm standard error of each metabolite during supplemented UV-A exposure (UV-A + PAR). Significant difference between control ( $0 \mathrm{~h}$ ) and UV-A exposure (2, 6, 12, 24 and $48 \mathrm{~h}$ ) was measured using a two-sample $\mathrm{t}$-Test with equal variance; $*=0.05>p \geq 0.01, * *=$ $0.01>p \geq 0.001$ and $* * *=p<0.001$. 


\subsubsection{UV-A vs. UV-B vs. PAR only exposure}

It is widely researched the effect of UV-B on cyanobacteria with less focus on UV-A exposure. UV-A and UV-B have different effects on photosynthetic organisms and mechanisms of protection will vary during supplemented UV-A and UV-B.

By comparing the UV-A results described (Section 3.2.3) with the UV-B (Section 3.2.2) and PAR only (Section 2.4.2) time-series experiments differences were observed. PAR only had the lowest overall number of significant peaks across the time-series comparing $0 \mathrm{~h}$ to each time point but, showed the highest percentage of elevated metabolite levels (Figure 49, Table 19).

Supplemented UV-B showed an increasing amount of significant peaks reducing in levels with increasing length of UV-B. $48 \mathrm{~h}$ of UV-B showed the highest amount of significance with 146 significant peaks, of those reduced in levels (0 vs. $48 \mathrm{~h}$ ) compared to the 63 decreasing in normalised abundance after $2 \mathrm{~h}$ of UV-B (Table 24).

UV-A showed the majority of significantly different peaks increasing in metabolite levels up to 24 h (positive $\log 2 \mathrm{FC}$, Table 23).

Table 24: Comparison of statistically significant $(p<0.05)$ peaks and number of those significant peaks with a positive or negative $\log 2 \mathrm{FC}$ during supplemented UV-B exposure. A positive $\log 2 \mathrm{FC}$ indicates an increase in metabolite level and a negative $\log 2 \mathrm{FC}$ value indicates a decrease in metabolite level. Both significance and $\log 2 \mathrm{FC}$ was calculated comparing control $(0 \mathrm{~h})$ and 2, 6, 12, 24 and $48 \mathrm{~h}$ of UVB exposure.

\begin{tabular}{cccc}
\hline $\begin{array}{c}\text { UV-B exposure } \\
\text { (h) }\end{array}$ & $\begin{array}{c}\text { No. of peaks, } \\
\boldsymbol{p}<\mathbf{0 . 0 5}^{\mathbf{a}}\end{array}$ & $\begin{array}{c}\text { No. of peaks, } \\
\text { positive log2FC }\end{array}$ & $\begin{array}{c}\text { No. of peaks, } \\
\text { negative log2FC }\end{array}$ \\
\hline 0 vs. $\mathbf{2}$ & 96 & 33 & 63 \\
0 vs. $\mathbf{6}$ & 98 & 29 & 69 \\
0 vs. $\mathbf{1 2}$ & 110 & 39 & 71 \\
0 vs. $\mathbf{2 4}$ & 105 & 27 & 78 \\
0 vs. $\mathbf{4 8}$ & 146 & 29 & 117 \\
\hline
\end{tabular}

${ }^{\mathrm{a}}=$ Significant difference was calculated using a two sample t-Test with equal variance. 


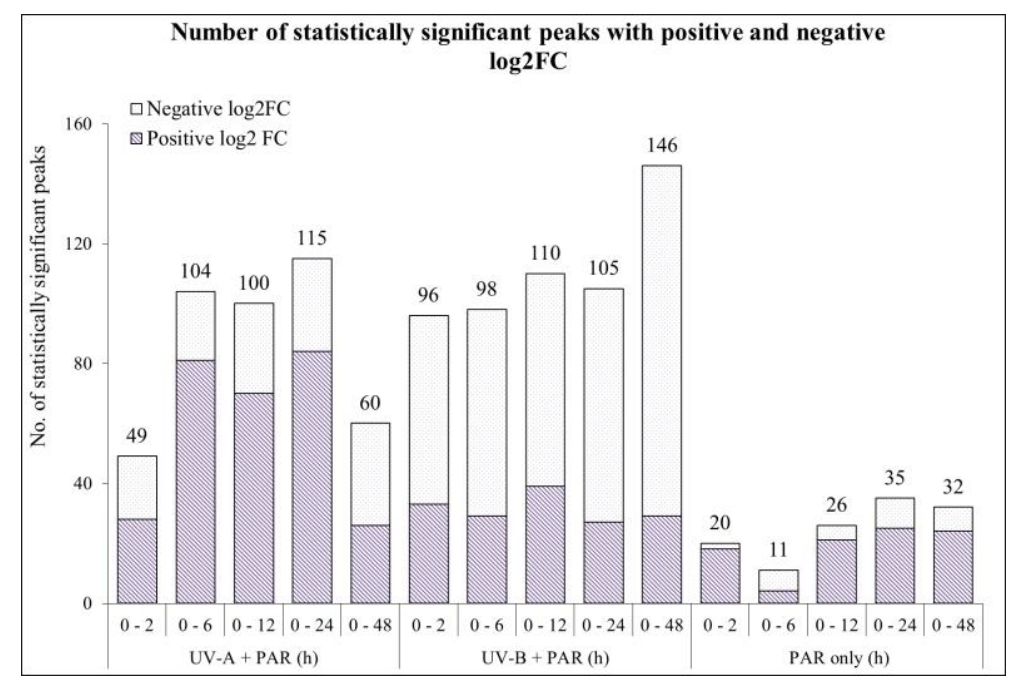

Figure 49: Total number of statistically significant peaks $(p<0.05)$ with positive and negative $\log 2 \mathrm{FC}$ comparing control $(0 \mathrm{~h})$ to each time point $2,6,12,24$ and $48 \mathrm{~h}$ of UV exposed (UV-A + PAR or UV$\mathrm{B}+\mathrm{PAR}$ ) and PAR only experiments. Significance was calculated using a two- sample t-Test. Diagonal stipes represents positive $\log 2 \mathrm{FC}$ and spots represents negative $\log 2 \mathrm{FC}$.

Eight (8) common metabolites were identified between supplemented UV-A, supplemented UV-B and PAR only exposure including glu, ser, palmitic acid and stearic acid. Using a one-way ANOVA-single factor, comparisons of $\log 2 \mathrm{FC}$ of the common metabolites (control, $0 \mathrm{~h}$ vs. each time point) between the three treatments were investigated (Figure 50).

Glu showed significance between the three treatments when comparing $\log 2 \mathrm{FC}$ at 0 vs. $12 \mathrm{~h}(\mathrm{~F}(2,6)=6.77, p<0.05), 0$ vs. $24 \mathrm{~h}(\mathrm{~F}(2,6)=32.70, p<0.001)$ and 0 vs. $48 \mathrm{~h}$ $(\mathrm{F}(2,6)=9.60, p<0.05)$ with the post-hoc t-Test (Two-sample assuming equal variances) showing significantly elevated levels in PAR only cultures compared to UV-A and UV-B with no significant differences observed between UV-A and UV-B. Ser showed significance comparing $\log 2 \mathrm{FC}$ between 0 vs. $48 \mathrm{~h}(\mathrm{~F}(2,6)=11.86$, $p<0.01$ ). Significancly reduced ser levels were observed in UV-B treated cultures (UVA vs. UV-B, $p<0.05$; PAR vs. UV-B, $p<0.05)$ with no significance comparing supplemented UV-A and PAR. 

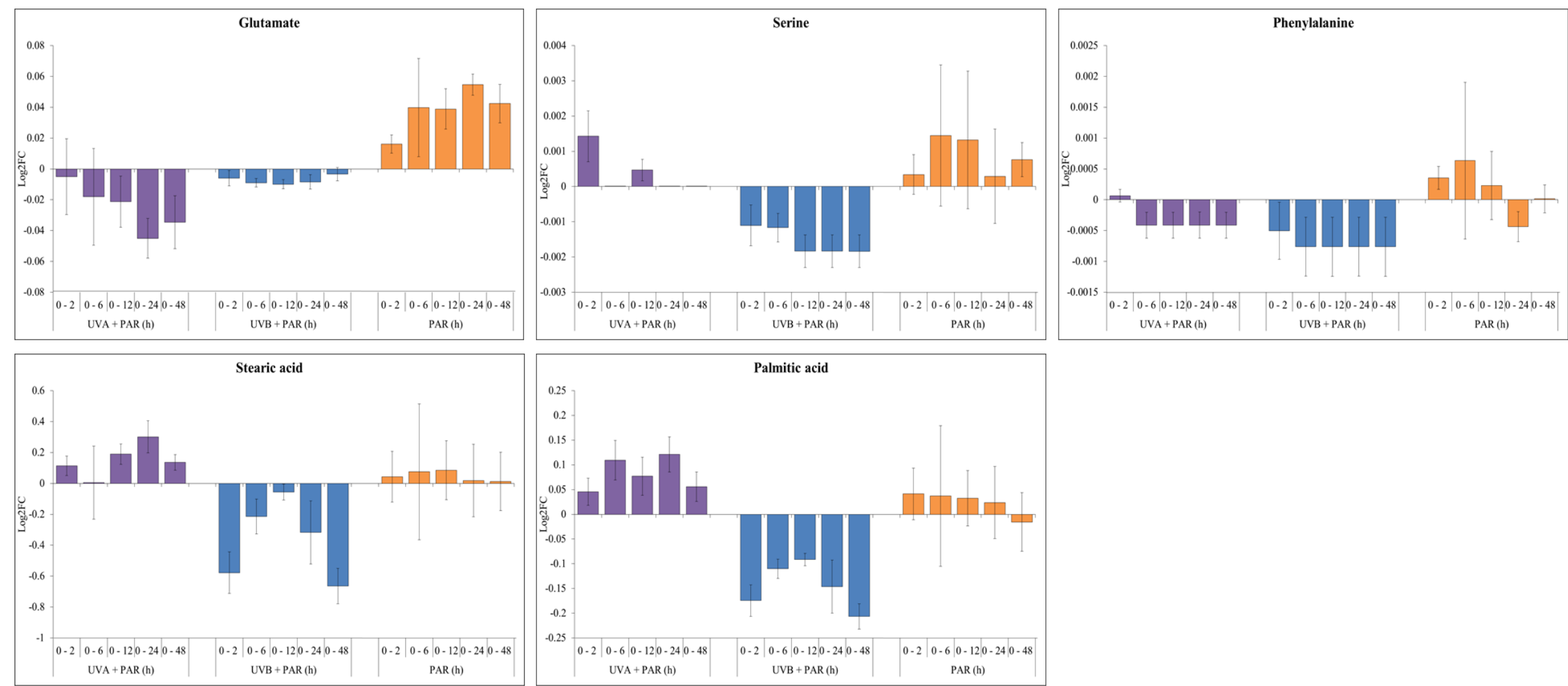

Figure 50: Log $2 \mathrm{FC}$ of commonly identified metabolites using GC-MS analysis during supplemented UV-A (purple), supplemented UV-B (blue) and PAR only (orange) exposure comparing $0 \mathrm{~h}$ and each time point $(2,6,12,24$ and $48 \mathrm{~h})$. A positive $\log 2 \mathrm{FC}$ indicates an increase in metabolite levels, and a negative log2 $\mathrm{FC}$ shows a decrease in metabolite levels. 
Palmitic acid shows significanc between the three treatments comparing 0 vs $2 \mathrm{~h}$ (F $(2,6)=10.59, p<0.05), 0$ vs. $24 \mathrm{~h}(\mathrm{~F}(2,6)=5.79, p<0.05)$ and 0 vs. $48 \mathrm{~h}(\mathrm{~F}(2,6)=$ $10.89, p<0.05)$. The t-Test results showed no significance between $\log 2 \mathrm{FC}$ of UV-A and PAR only exposure experiments but significant reduction in levels was observed between UV-A and UV-B as well as PAR and UV-B. Stearic acid shows significance at 0 vs. $2 \mathrm{~h}(\mathrm{~F}(2,6)=9.71, p<0.05)$ and 0 vs. $48 \mathrm{~h}(\mathrm{~F}(2,6)=10.22, p<0.05)$. A significant reduction was again observed in the UV-B exposed cultures (UV-A vs UVB and PAR only vs. UV-B).

An additional two metabolites were identified in both supplemented UV-A and PAR only exposure, leu and sucrose (Figure 51). Leu showed opposite $\log 2 \mathrm{FC}$ patterns with supplemented UV-A (UV-A + PAR) decreasing in normalised abundance compared to $0 \mathrm{~h}$, with these metabolites increasing in abundance during the PAR only time-series (with no significance, $p>0.05$ ) Sucrose also showed a similar opposite pattern with decreasing abundance with increasing length of UV-A and increasing during PAR only exposure ( 0 vs. 12 h, $p<0.05$; 0 vs. 24 h, $p<0.01$ and 0 vs. 48 h, $p<0.01)$.
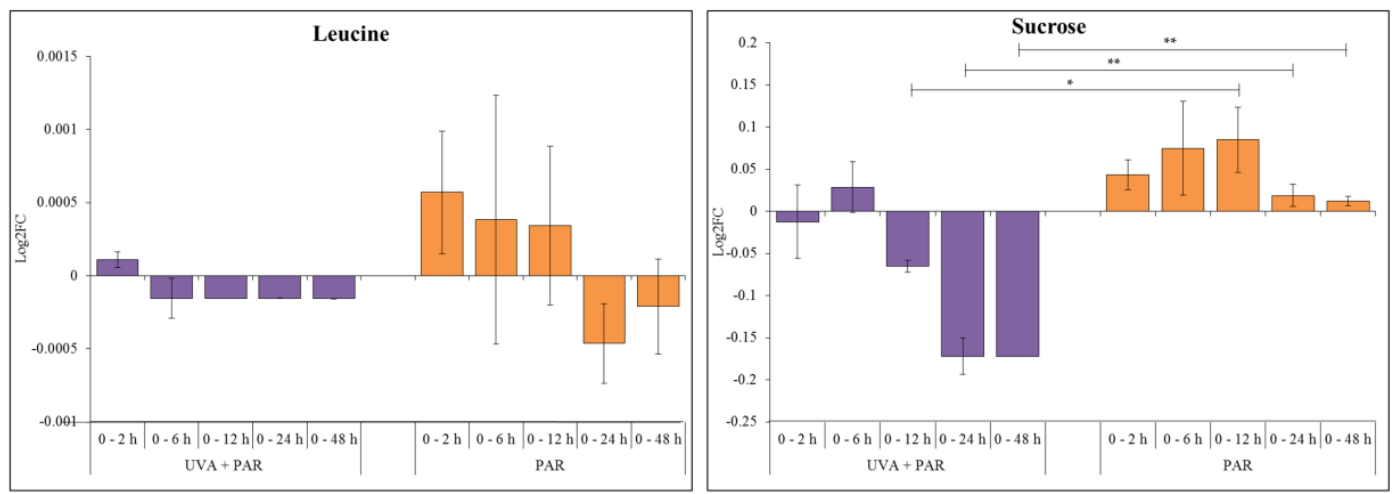

Figure 51: $\log 2$ FC of commonly identified metabolites using GC-MS analysis during supplemented UV-A (purple) and PAR only (orange) exposure comparing $0 \mathrm{~h}$ and each time point (2, 6, 12, 24 and $48 \mathrm{~h}$ ). A positive $\log 2 \mathrm{FC}$ indicates an increase in metabolite levels, and a negative log2FC shows a decrease in metabolite levels. Significant differences between $\log 2 \mathrm{FC}$ values was calculated using a two-sample t-Test, $*=0.05>p \geq 0.01, * *=0.01>p \geq 0.001$ and $* * *=p<0.001$.

These opposite $\log 2 \mathrm{FC}$ patterns are also observed between supplemented UV-A and supplemented UV-B time-series data (Figure 52). This includes significantly reduced oxalate levels ( 0 vs. 6 h, $p<0.01 ; 0$ vs. 24 h, $p<0.01$ ), lactate levels ( 0 vs. 12 h, $p<0.05$ ), mystiric acid ( 0 vs. 6 h, $p<0.05$; 0 vs. 24 h, $p<0.05$ and 0 vs. 48 h, $p<0.05$ ) and mannose levels ( 0 vs. $24 \mathrm{~h}, p<0.05)$ during UV-B exposure compared to UV-A. 

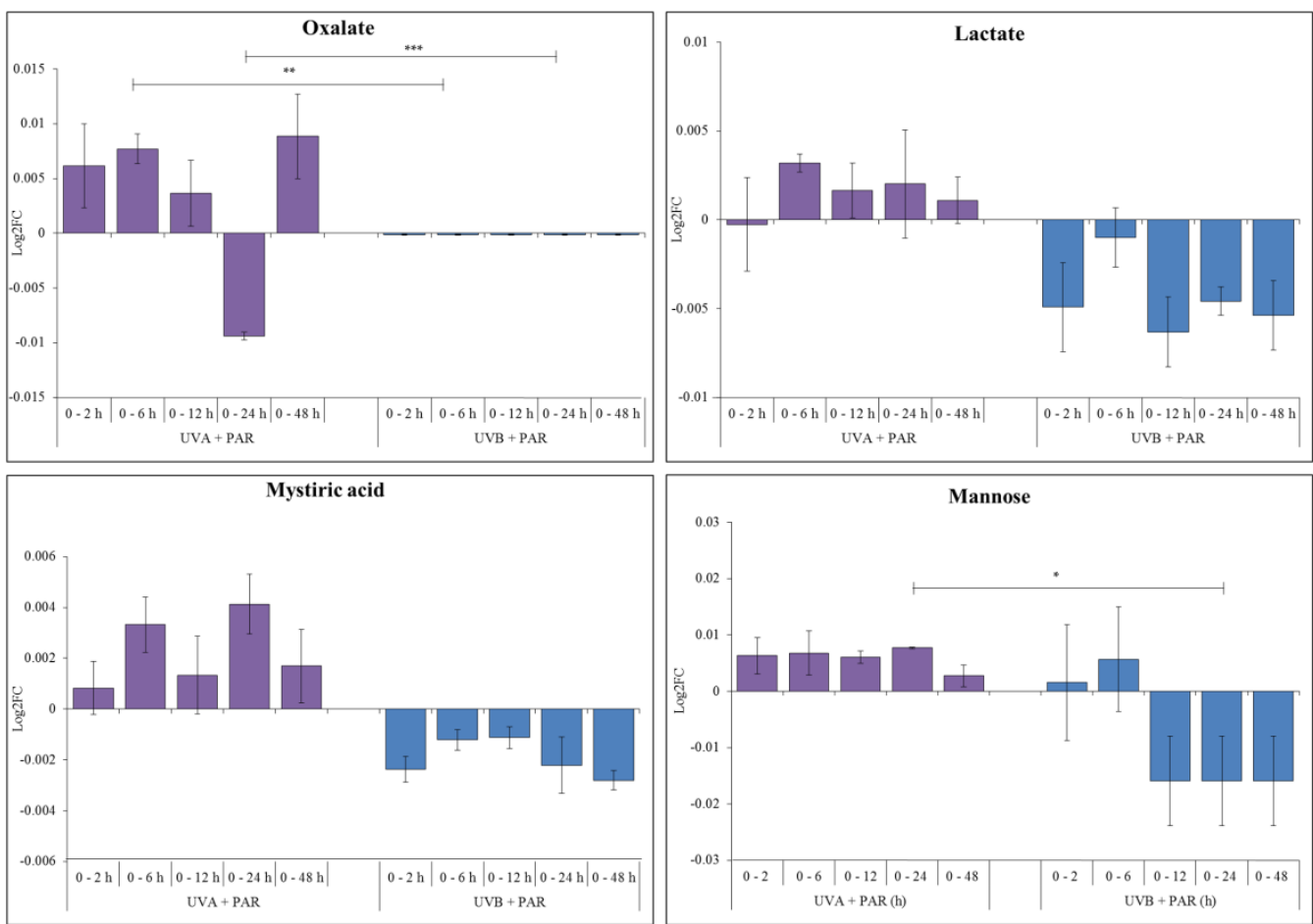

Figure 52: $\log 2 \mathrm{FC}$ of commonly identified metabolites using GC-MS analysis during supplemented UV-A (purple) and UV-B (blue) exposure comparing no UV (0 h) and each time point $(2,6,12,24$ and $48 \mathrm{~h})$. A positive $\log 2 \mathrm{FC}$ indicates an increase in metabolite levels, and a negative $\log 2 \mathrm{FC}$ shows a decrease in metabolite levels. Statistical significance between $\log 2 \mathrm{FC}$ values was calculated using a two-sample t-Test, $*=0.05>p \geq 0.01, * *=0.01>p \geq 0.001$ and $* * *=$ $p<0.001$. 


\subsection{Discussion}

\subsubsection{The effect of UVR on metabolite levels in $C$. fritschii}

UV-B exposure is known to reduce growth, photosynthesis and nitrogen fixation in cells to divert energy from key primary pathways to adaptive mechanisms such as; the production of secondary metabolites, MAAs; antioxidant production and DNA/protein repair [119]. These results showed no significance $(p>0.05)$ across the time series suggesting acclimation of cells to UV-B where damage to photosynthetic systems are counterbalanced by repair and mitigation strategies. A reduction in carotenoid concentration (Figure 41A) was observed after $2 \mathrm{~h}(p<0.05)$ due to damage to photosystems caused by UV-B. Accumulation of total carotenoids could be indicative of antioxidant activity as a response to ROS production $[6,7]$.

A reduction in glucose, pyruvate and lactate (Figure 44) could indicate a reduction in $\mathrm{CO}_{2}$ fixation via photosynthesis and further biochemical processes. This could be due to the reduced production of ATP and $\mathrm{NADPH}_{2}$ from photosynthesis [120].

The 13 selected intracellular metabolites (Figure 44) were reduced across the UV-B time series indicating the reduction of cellular processes. A decrease in phe and tyr could be due to their role as precursors to many secondary metabolites such as aromatic nitrogen containing alkaloids [10]. M-gly and shinorine are produced via a combination of the shikimate/pentose phosphate pathway which also involves the addition of gly and ser to form the final MAAs [6,28]. The reduction of these amino acids coincides with an increase in MAA levels (Figure 41B).

5-oxoproline and glu are involved in glutathione metabolism. 5-oxoproline reduction could be due to its interconversion into glutamate which is further converted into the antioxidant glutathione [121]. Glu is also produced from the assimilation of nitrogen during nitrogen fixation which is reduced during UV-B exposure [120]. C. fritschii is a nitrogen fixating species via formation of specialised cells known as heterocysts. During these experiments, $C$. fritschii was not grown under nitrogen limiting conditions.

Pro has been studied in many UV-B experiments involving different photosynthetic organisms and it's accumulation is thought to have a role in stress response by providing additional defense as a ROS scavenger and molecular chaperone [122-124]. Accumulation of proline has been observed in Nostoc punctiforme during $24 \mathrm{~h}$ of UV- 
A stress [52], in the model plant organism Arabidopsis after $24 \mathrm{~h}$ of UV-B treatment [96], and also in C. fritschii after 24 h of UV-B exposure within this study (Figure 44).

The movement of metabolites and substrates between cells and their surrounding environment (or vice versa) can occur via passive and active uptake and efflux systems. Reactions can also occur on cell surface membranes and as transformations of media components [125]. Identification of extracellular metabolite uptake and release from cyanobacteria is therefore a complex process due to the high turnover rates of intracellular processes [69]. Extracellular metabolites can be released during stress and as by-products of intracellular reactions [54]. Sugars such as galactose, arabinose, lyxose and xylose are produced/released during UV-B stress. As expected, this was seen in this UV-B experiment (Figure 45). Increased production of sugars is a strategy to avoid high intensity UVR. They can mitigate UV-B stress by providing an extra layer around the cells to absorb the harmful UV-B [24].

7 metabolites from the identified biologically relevant pool were found in both intraand extracellular metabolite samples (Figure 46). The fatty acid mystiric acid shows a similar pattern of reduced abundance in intracellular samples with concomitant decrease in extracellular samples with increasing length of UV-B. This could demonstrate the reduction in biosynthesis or the increase in intracellular turnover rate with an increase in the release of this metabolite into the supernatant [125]. As this is seen during UV-B exposure, the hypothesis of a reduction in biosynthesis is considered. Ethanolamine, involved in glycerophospholipid metabolism, and 2oxobutanoate, involved in amino acid biosynthesis show opposite patterns with intracellular levels decreasing with concomitant increase in extracellular levels. This could should evidence of metabolite flux, a movement from within cells to the supernatant which can occur via passive or active secretion mechanisms [125]. These results can help create hypotheses and give an insight into the production and movement of metabolites from within cells to their release into the supernatant. For verification, flux analysis would need to be conducted using isotopic-labelled metabolic intermediates to follow labelled metabolites within the cells to their potential release [42].

It is widely researched the effect of UV-B on cyanobacteria with less focus on UV-A exposure. UV-A and UV-B have differing wavelengths and therefore have varying 
effects on photosynthetic organisms and mechanisms of protection vary. However, they both indirectly damage cells via the production of ROS [27,126]. An increase in carotenoid levels (Figure 47B) was observed with increasing length of UV-A, this is consistent with previous experiments during UV-B exposure (Section 3.2.2). This accumulation could be due to the increased ROS production caused by increasing length of UV where carotenoids act as antioxidants. An initial decrease in dry weight (Figure 47A) could indicate the shock response of $C$. fritschii to initial UV-A exposure. An increase in biomass was then observed between $6 \mathrm{~h}$ and $48 \mathrm{~h}$ showing the acclimation of cells to UV-A, as supported by the GC-MS data, where less significance is seen between $0 \mathrm{~h}$ and $48 \mathrm{~h}$ of supplemented UV-A (PAR + UV-A, Figure 49).

The metabolites, glu and phe showed negative $\log 2 \mathrm{FC}$ in both supplemented UV-A and UV-B compared to the positive $\log 2 \mathrm{FC}$ during PAR only exposure (Figure 50) thus suggesting the roles of these amino acids during UVR stress. A decrease in phe levels is consistent with the need for aromatics such as phenylpropanoids which can absorb harmful UVR due to their unsaturated nature and for protein synthesis [10]. Glu is involved in glutathione synthesis which has a role as an antioxidant and protects cells from ROS, an example includes the conversion of $\mathrm{H}_{2} \mathrm{O}_{2}$ into $\mathrm{H}_{2} \mathrm{O}$ to prevent the formation of $\bullet \mathrm{OH}[121]$.

The soluble sugar sucrose, a disaccharide consisting of glucose and fructose, has been seen to have a role in stress tolerance in both plants and cyanobacteria $[99,127]$ which is consistent with the opposite effect between UV-A exposure and PAR only exposure (Figure 51).

UV-A and PAR only exposure have opposite effects on the saturated fatty acids stearic acid and palmitic acid compared to UV-B, with the accumulation during UV-A and PAR only and a decrease during UV-B exposure. This trend is also seen with mystiric acid (Figure 52, comparing UV-A and UV-B). Saturated fatty acids provide energy for the rebuilding of photosynthetic apparatus which could be damaged directly during UV-B exposure [99].

Overall, these results show that supplemented UV-A allows for better re-organisation of metabolism in $C$. fritschii cells compared to UV-B. Both cause changes in metabolite levels associated with UVR stress response such as phe, glu and sucrose. 
Reductions in fatty acids, oxalate, mannose and lactate levels could be associated with UV-B stress response due to its direct and indirect effects.

\subsection{Conclusion}

In summary, an untargeted GC-MS workflow was used to evaluate intra- and extracellular metabolites under supplemented UV-B (PAR + UV-B) and intracellular metabolites during supplemented UV-A (PAR + UV-A) exposure. Most significantly during UV-B exposure a reduction of intracellular metabolites such as the amino acids, tyr, phe, ser, gly and glu and the accumulation of pro, which to our knowledge has not been previously reported in $C$. fritschii. Significantly a higher proportion of metabolites increased in levels during UV-A exposure compared the UV-B study. An accumulation of the fatty acids; eicosanoic acid, stearic acid and palmitic acid was observed. The metabolites phe, glu and sucrose could be affected by both UV-A and UV-B whereas other metabolites such as fatty acids are affected by the UV-B portion of UVR only.

Although a time series analysis was conducted, this only represents a minuscule proportion of the true changes within the metabolome. This study is important to build on experimental data already available for cyanobacteria and other photosynthetic organisms exposed to UV-B and UV-A. It is important to investigate the changes in primary metabolites in order to understand secondary metabolite production as previously discussed. Manipulating abiotic stress such as UV radiation can be used to enhance production of useful metabolites for uses in industrial biotechnology $[16,21,22]$. Further studies are needed to understand and verify these processes within cyanobacteria to aid in the understanding of UV stress adaptation at the metabolite level. 


\section{Chapter 4: Acclimation vs. Shock: The Stress Response of Chlorogloeopsis fritschii, PCC 6912, to UV Exposure}

\section{Aims}

- Evaluation of the effect of acclimatising C. fritschii cultures to low levels of UV (acclimation phase) followed by exposure to high UV (shock phase).

- To investigate if cultures can tolerate the higher UV levels after acclimation.

- To evaluate the metabolite level changes over the time-series and comparing to non-acclimatised cultures (UV shock only) using GC-MS. 


\section{Introduction}

Adaptation of cyanobacteria to different abiotic factors is important for their survival in a variety of extreme habitats. They have many strategies to overcome harmful environmental stimuli such as rearrangement of metabolism for efficient energy uses as well as production of unique secondary metabolites [9]. The ability to manipulate abiotic stressors to increase production of specific metabolites can be exploited for biotechnological uses in industry and enhance stress tolerance in specific strains [128].

Cyanobacterial salt acclimation has been widely studied for industrial growth in seawater [127,129] as well as; high light acclimation [130], far-red light acclimation [131] and UV-B acclimation [113].

Selection of robust and tolerant cyanobacterial strains is essential for their use in industrial biotechnology [132]. Many strategies have been investigated such as use of extremophilic strains [133], genetic manipulation such as heterologous expression of heat shock proteins in Synechococcus elongates [134], supplementation of media [135], and pre-treatment with sub-lethal doses of specific abiotic stress condition as seen in rice plants [136] and in Anabaena doliolum where pre-treatment of increased temperature showed reduction in UV-B toxicity [137].

As previously described, UV affects cyanobacteria in a variety of ways. From targeting biomolecules directly to promoting the production of harmful ROS levels. They have various tolerance mechanisms to overcome the damage caused by UV [24]. This includes the accumulation of unique secondary metabolites such as carotenoids and MAAs [113].

The aim of the chapter is to evaluate $C$. fritschii's stress tolerance to UV (UV-A and UV-B) at the metabolite level and to build on results from the previous stress response chapter (Chapter 3). The effect of pre-treatment with low dose UV (acclimation phase) followed by increased levels (shock phase) to investigate if tolerance is enhanced to higher UV levels. These experiments will be used to evaluate if $C$. fritschii has acclimatised to the UV sufficiently to withstand the higher levels of UV (shock). 


\subsection{Experimental design}

\subsubsection{Acclimation and shock vs. shock only experiments}

All experiments were carried out under continuous PAR $\left(15 \mu \mathrm{mol} \mathrm{m} \mathrm{m}^{-2} \mathrm{~s}^{-1}\right)$, a temperature of $27 \pm 2{ }^{\circ} \mathrm{C}$ and shaking at $100 \mathrm{rpm}$ for even UV exposure of cells. For UV exposure, cultures were transferred into Quartz Erlenmeyer flasks (H. Baumbach \& CO.LTD, Suffolk). UV-A was supplied by a Philips TL-D $18 \mathrm{~W}$ black light blue fluorescent tube (315-400 nm, centred at $360 \mathrm{~nm}$ ) and UV-B was supplied by a Philips TL 20W UVB Broadband fluorescent tube (290-315 nm, centred at $310 \mathrm{~nm}$ ).

\subsubsection{Acclimation and shock}

After 6 days of pre-growth (Section 2.1), triplicate experimental $C$. fritschii cultures were acclimatised to UV-A $\left(11.5 \mu \mathrm{mol} \mathrm{m} \mathrm{m}^{-2} \mathrm{~s}^{-1}\right)$ or UV-B $\left(3 \mu \mathrm{mol} \mathrm{m} \mathrm{m}^{-2}\right)$ for $24 \mathrm{~h}$ with constant PAR $\left(15 \mu \mathrm{mol} \mathrm{m} \mathrm{m}^{-2}\right)$ followed by shock exposure of $20 \mu \mathrm{mol} \mathrm{m} \mathrm{m}^{-2} \mathrm{~s}^{-1} \mathrm{UV}-\mathrm{A}$ or $5.5 \mu \mathrm{mol} \mathrm{m} \mathrm{m}^{-2} \mathrm{UV}-\mathrm{B}$ for a further $24 \mathrm{~h}$ (Table 25). Samples were harvested at 0 , 24, 24.5, 25.5 and $48 \mathrm{~h}$ for dry weight measurements (Section 1.2), pigment (Section 1.5.1), MAA (Section 1.4.2-1.4.3) and GC-MS analysis (Sections 1.3.2 - 1.3.6).

\subsubsection{Shock only}

C. fritschii cultures were grown with PAR $\left(15 \mu \mathrm{mol} \mathrm{m} \mathrm{m}^{-2} \mathrm{~s}^{-1}\right)$ only for $24 \mathrm{~h}$. Cultures were then exposed to UV-A $\left(20 \mu \mathrm{mol} \mathrm{m} \mathrm{s}^{-2}\right)$ or UV-B $\left(5.5 \mu \mathrm{mol} \mathrm{m} \mathrm{m}^{-2} \mathrm{~s}^{-1}\right)$ for a further $24 \mathrm{~h}$ (Table 25). Samples were harvested at 0, 24, 24.5, 25.5 and $48 \mathrm{~h}$ for dry weight measurements (Section 1.2), pigment (Section 1.5.1), MAA (Section 1.4.2-1.4.3) and GC-MS analysis (Sections 1.3.2 - 1.3.6). 
Table 25: PAR, UV-B and UV-A intensities during acclimation and shock experiments. PAR + UV-B treatments were carried out separately from PAR + UV-A.

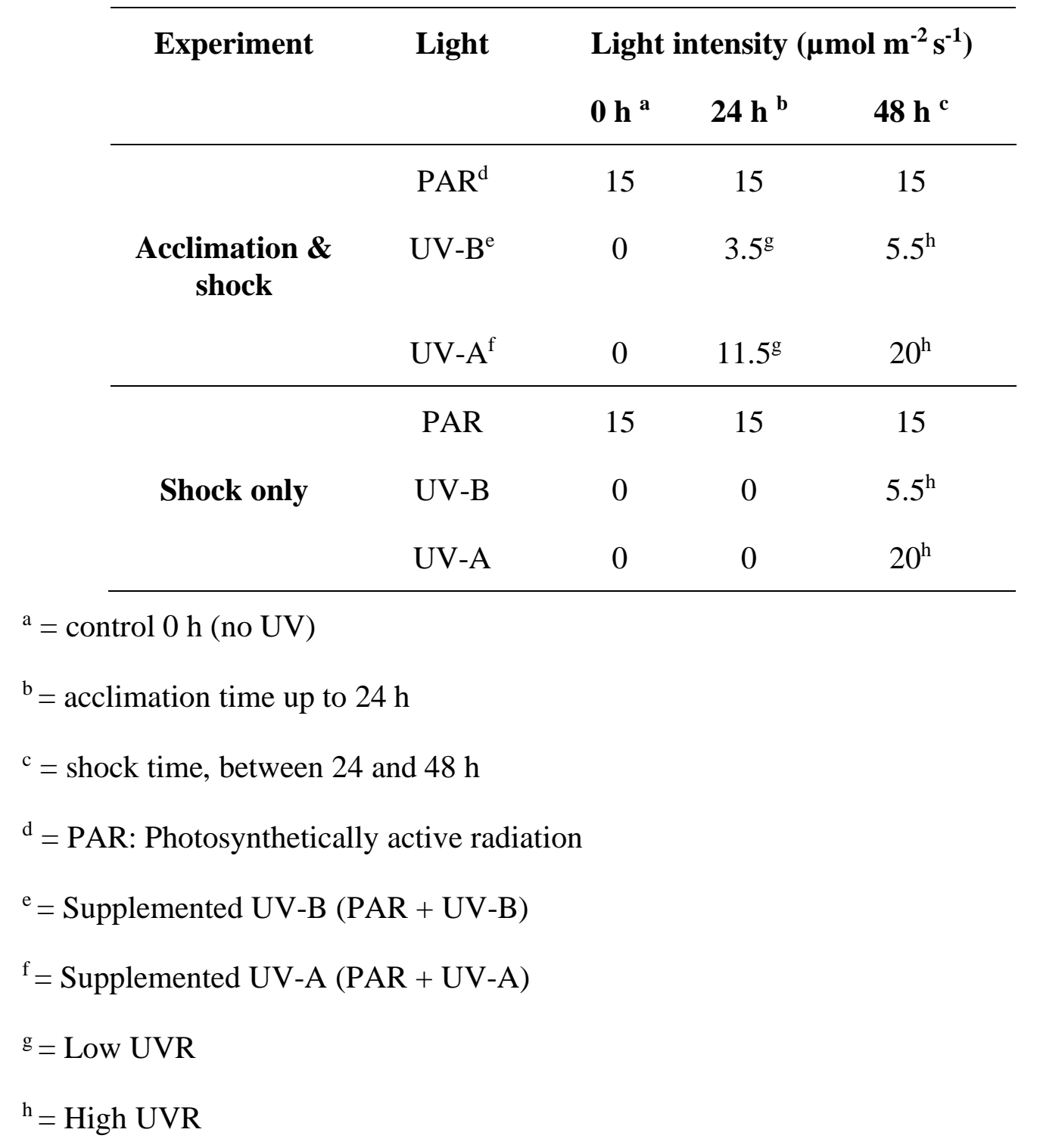




\subsection{Results}

\subsubsection{UV-B acclimation and shock vs. shock only}

\subsubsection{Dry weight, pigment analysis and production of MAAs}

During UV-B acclimation a decrease in dry weight (Figure 53A, $\mu=-0.0018$, Figure 54) was observed compared to non-acclimatised control cultures (Figure 53B, $\mu=$ 0.0047) which increased in dry weight (no significance, $p<0.05$ ). Both acclimatised (low UV-B) and non-acclimatised (No UV-B) cultures decreased in growth after 0.5 $\mathrm{h}$ of high UV-B and acclimatised cultures decreased further after $1.5 \mathrm{~h}$ to a minimum dry weight of $116.7 \mathrm{mg} \mathrm{L}^{-1}$, while non-acclimatised cultures increased in growth up to $145.6 \mathrm{mg} \mathrm{L}^{-1}$. A significantly lower dry weight was measured after $1.5 \mathrm{~h}$ of shock in the acclimatised cultures compared to the non-acclimatised cultures (acclimation and shock vs. shock only; $25.5 \mathrm{~h}, p<0.05)$. After 24 h of shock UV-B exposure, cultures acclimatised to UV-B (Figure 53A) increased in growth up to a final dry weight measurement of $134.4 \mathrm{mg} \mathrm{L}^{-1}(\mu=0.0003$, Figure 54) whereas the cultures not acclimatised to UV-B (Figure 53B) decrease in dry weight to $131.1 \mathrm{mg} \mathrm{L}^{-1}$ during the $24 \mathrm{~h}$ shock phase $(24$ to $48 \mathrm{~h}, \mu=-0.0053)$.

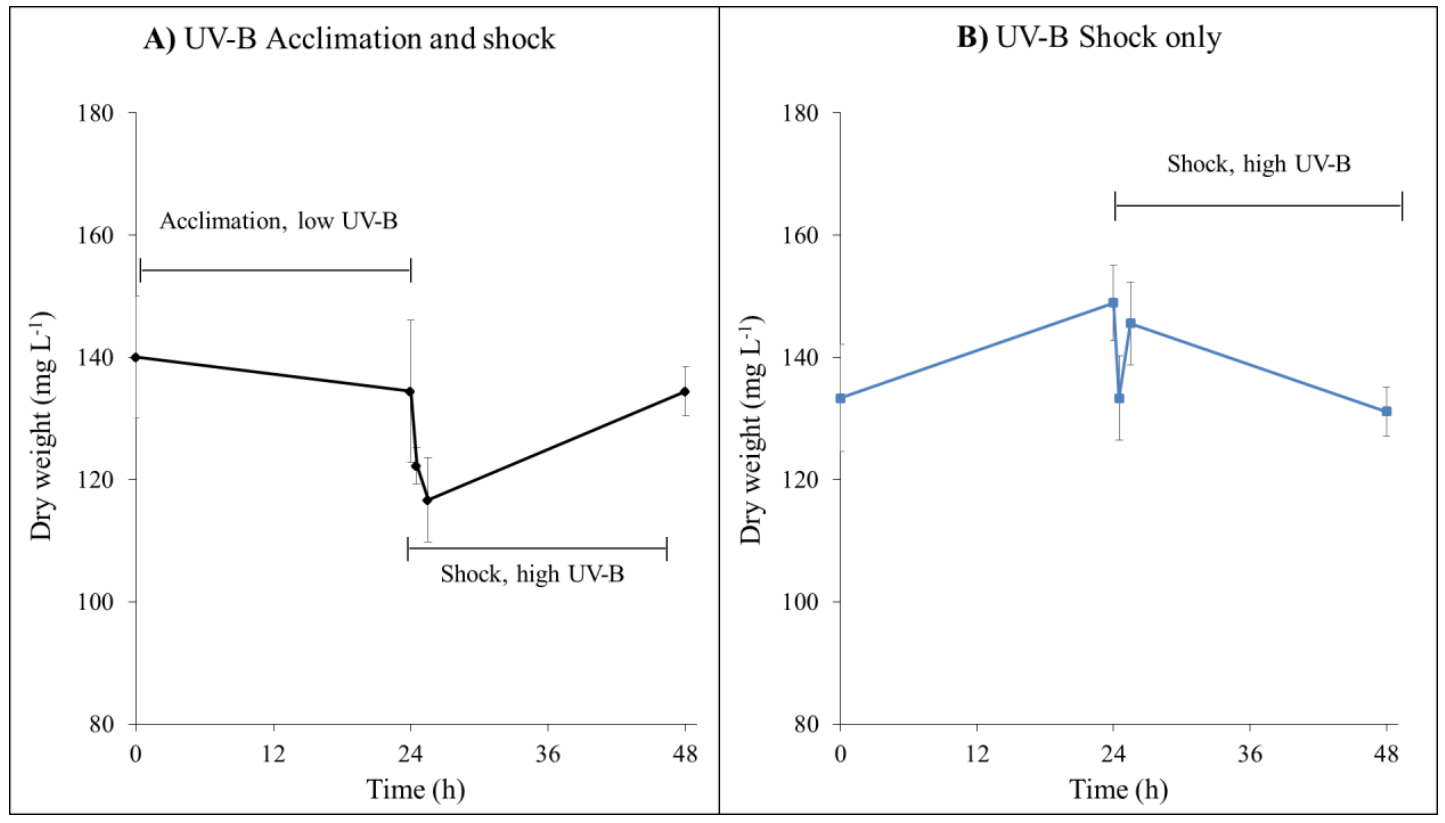

Figure 53: Dry weight analysis $\left(\mathrm{mg} \mathrm{L}^{-1}\right)$ of $C$. fritschii cultures during A) UV-B acclimation and shock and B) UV-B shock only. Data points are mean values of biological triplicates \pm SE. Significant differences were measured using a two-sample t-Test, $*=0.05>p \geq 0.01, * *=0.01>p \geq 0.001$ and $* * *=p<0.001$. 


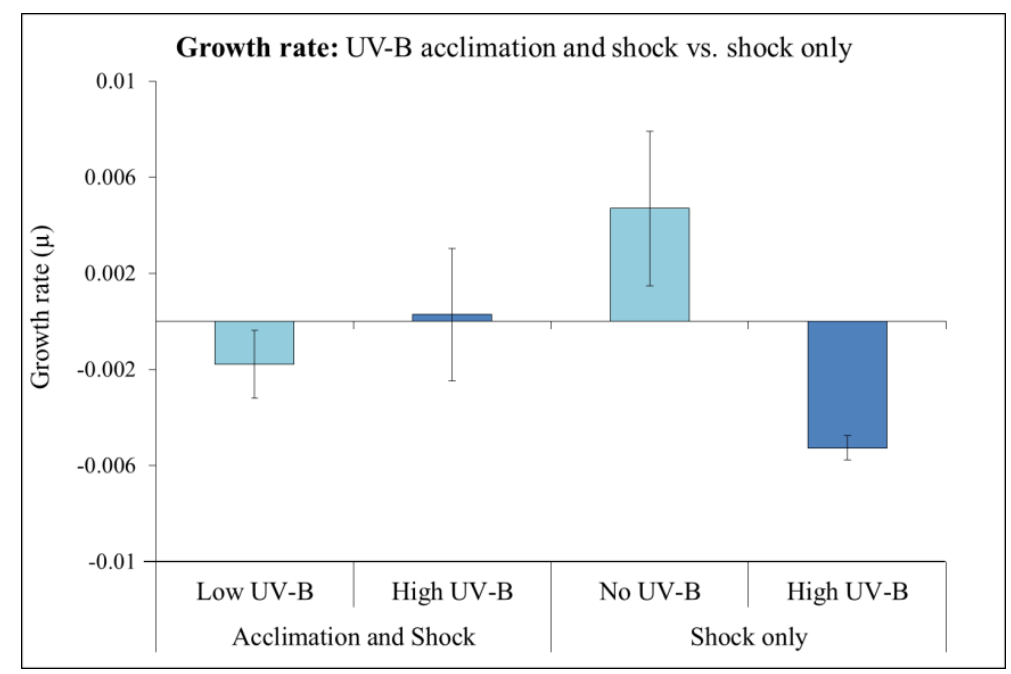

Figure 54: Growth rate $(\mu)$ of UV-B acclimation and shock vs. shock only of $C$. fritschii cultures. Low UV-B and no UV-B accounts for the growth rates between 0 and $24 \mathrm{~h}$ and high UV-B accounts for growth rate between 24 and $48 \mathrm{~h}$. Data represents mean growth rates of biological triplicates $\pm \mathrm{SE}$.

Pigment concentration showed an increase in levels of both carotenoid and chl $a$ content up to 1.5 and $4.7 \mu \mathrm{g} \mathrm{mg}^{-1}$ dry weight (DW) respectively at $25.5 \mathrm{~h}$ with a significant increase observed at $24.5 \mathrm{~h}$ (24 vs. $24.5 \mathrm{~h}, p<0.001)$ during acclimation and shock (Figure 55A). A statistical decrease in pigment was then observed between $25.5 \mathrm{~h}$ and $48 \mathrm{~h}$ during high UV-B (Figure 55A; 25.5 vs. $48 \mathrm{~h}, p<0.05$ ). During shock only UV-B exposure (Figure 55B) an initial increase in levels of chl $a$ and carotenoids was observed after $0.5 \mathrm{~h}$ of high UV-B to 2 and $6.5 \mu \mathrm{g} \mathrm{mg}^{-1} \mathrm{DW}$ respectively. A significant decrease in levels was then measured for both chl $a$ and carotenoids (24.5 vs. $48 \mathrm{~h}, p<0.05$ ) to 1.5 and $4.7 \mu \mathrm{g} \mathrm{mg}^{-1} \mathrm{DW}$. 


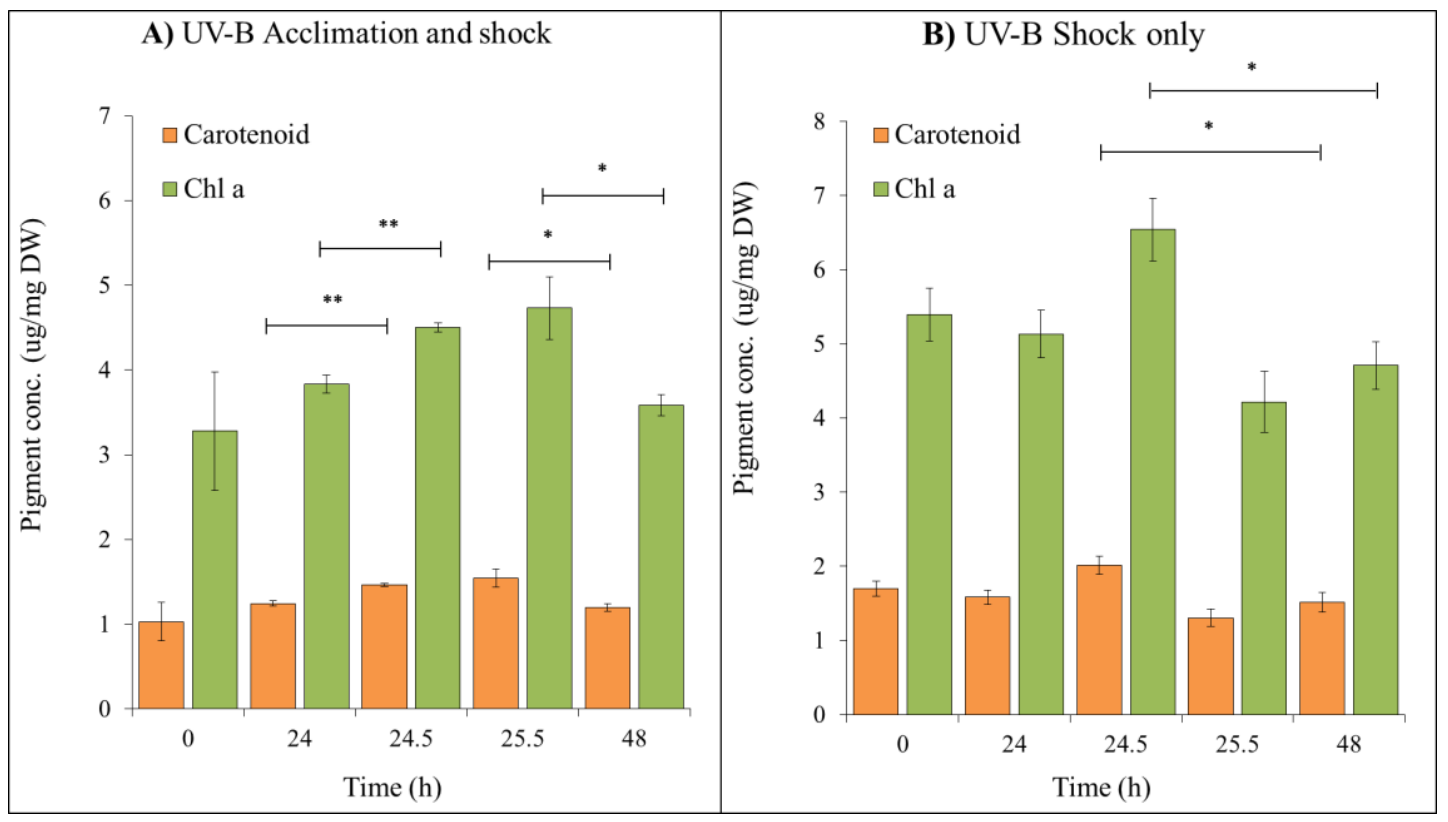

Figure 55: Pigment content ( $\mu \mathrm{g} / \mathrm{mg}$ dry weight) of $C$. fritschii cultures during A) UV-B acclimation and shock and B) UV-B shock only. Data points are mean values of biological triplicates \pm SE. Statistical significance was measured using a two-sample t-Test, $*=0.05>p \geq 0.01, * *=0.01>p \geq$ 0.001 and $* * *=p<0.001$.

An increase in both shinorine and m-gly ( $p<0.01,0$ vs. $24 \mathrm{~h}$ ) content was observed after $24 \mathrm{~h}$ of low UV-B exposure (Figure 56A) with further increases in content during high UV-B exposure (m-gly, 0 vs. 24.5 h, $p<0.001 ; 0$ vs. 48 h, $p<0.01$ ). Shinorine and m-gly were detected during high UV-B exposure only (Figure 56B) up to $48 \mathrm{~h}$ with a significant increase (m-gly, 0 vs. $25.5 \mathrm{~h}, p<0.01$; 0 vs. $48 \mathrm{~h}, p<0.01$ ). Comparing between treatments (acclimation and shock vs. shock only), significantly higher levels of m-gly were calculated at $24 \mathrm{~h}(p<0.01)$ and $24.5 \mathrm{~h}(p<0.01)$ in the acclimatised cultures. 


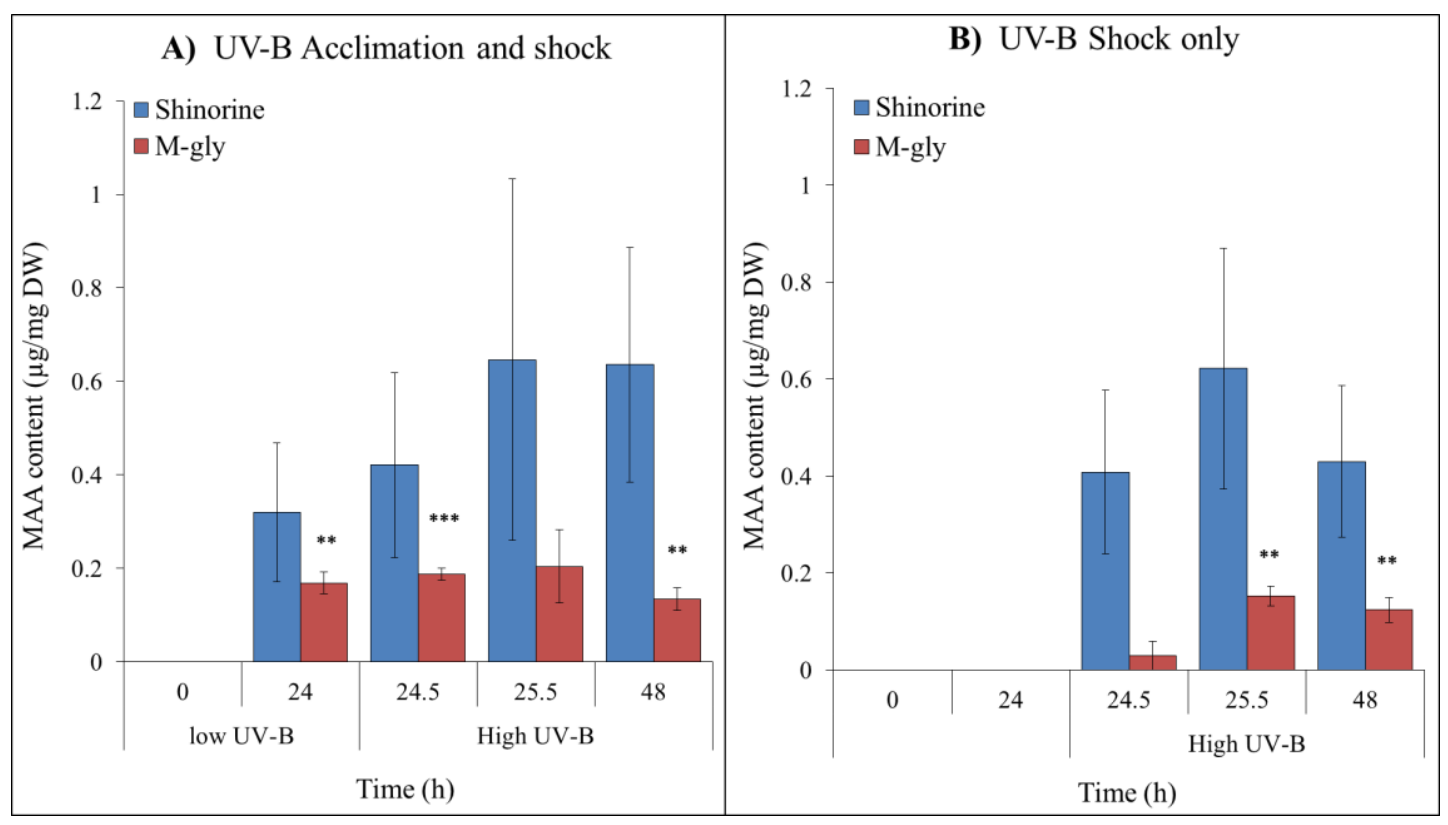

Figure 56: MAA content ( $\mu \mathrm{g} / \mathrm{mg}$ dry weight) of $C$. fritschii cultures during A) UV-B acclimation and shock and B) UV-B shock only. Data points are mean values \pm SE. Signifiacnt differences were measured using a two-sample t-Test comparing $0 \mathrm{~h}$ to each time point $(24,24.5,25.5$ and $48 \mathrm{~h})$, * = $0.05>p \geq 0.01, * *=0.01>p \geq 0.001$ and $* * *=p<0.001$.

\subsubsection{Intracellular metabolite levels}

\section{UV-B shock only}

During UV-B shock only, a total of 396 peaks were detected, 67 of those were identified and 329 were left unassigned. Initially, 171 significant peaks were detected using a two-sample t-Test with equal variance comparing 0 and $24 \mathrm{~h}$ (no UV-B). 43 of those peaks (11\% of total amount of peaks) significantly reduced in levels (negative $\log 2 \mathrm{FC}$ ) whereas 128 peaks (32\% of total) had significantly increased levels (positive $\log 2 \mathrm{FC})$. An increase in significant peaks (185 peaks) was observed by increasing the intensity of UV-B (high UV-B, 24 vs. 48 h). 53 (13\% of total) showed significantly elevated metabolite levels (positive $\log 2 \mathrm{FC}$ ) with 132 (33\% of total) showed a significant decresase in normalised abundances (negative $\log 2 \mathrm{FC}$ ) (Figure 57). 


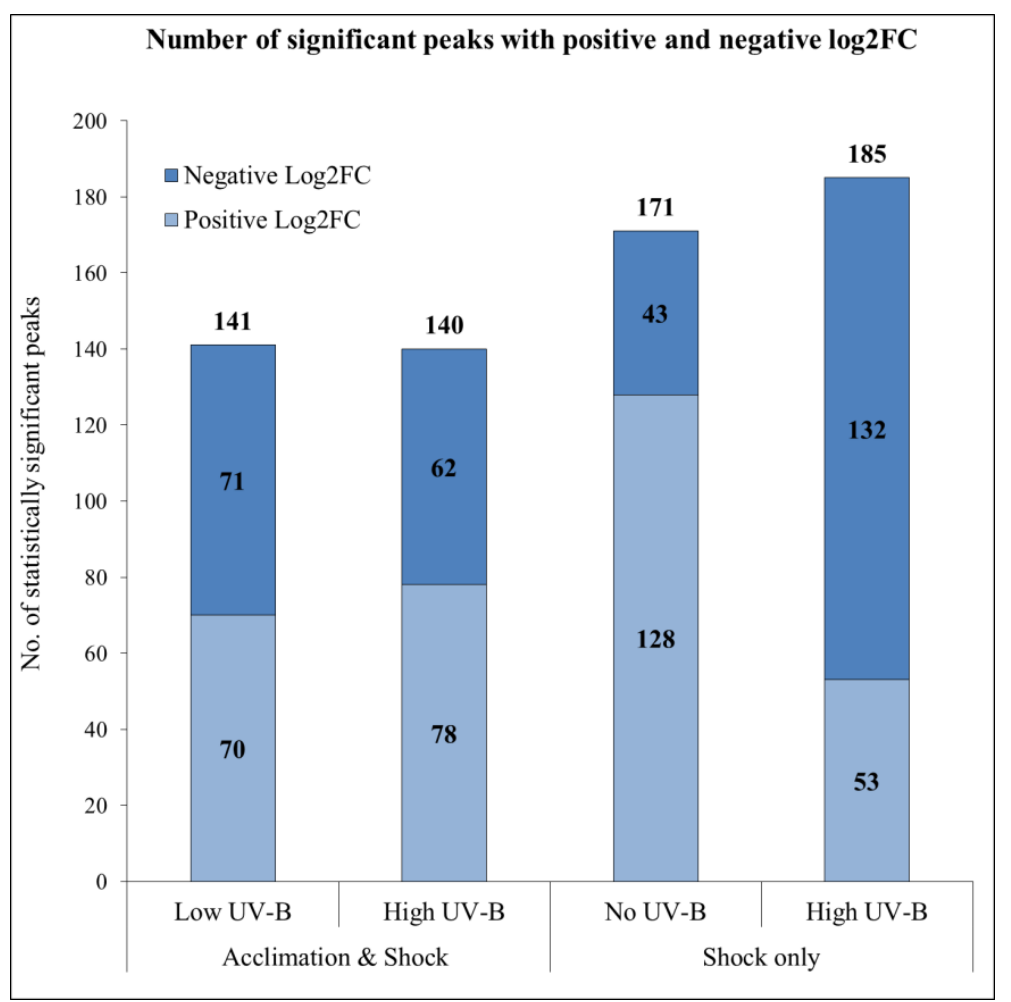

Figure 57: Number of detected peaks that show significant differences $(p<0.05)$ during acclimation and shock (total peaks $=428$ ) and shock only (total peaks $=396$ ) comparing 0 vs. $24 \mathrm{~h}$ (low UV-B or no UV-B) and 24 vs. $48 \mathrm{~h}$ (high UV-B) using a two-sample t-Test with equal variance. The percentage of significant peaks with positive and negative $\log 2 \mathrm{FC}$ comparing 0 vs. $24 \mathrm{~h}$ (low UV-B or no UV-B) and 24 vs. $48 \mathrm{~h}$ (high UV-B) is also presented.

\section{UV-B acclimation and shock}

During UV-B acclimation and shock a total of 428 peaks were detected, 101 of those were identified and 327 left unassigned. 141 significant peaks were observed during the acclimation phase of low UV-B exposure ( 0 vs. 24 h; $33 \%$ of total peaks). 70 peaks (17\% of total) showed increasing normalised abundances (positive log2FC) and 71 (16\% of total) showed a decrease in levels (negative $\log 2 \mathrm{FC}$ ). After shock UV-B exposure (high UV-B), the number of significant peaks ( 24 vs. 48 h) remained the same $(33 \%)$. An increase in peaks elevating in levels (18\%, 78 peaks) and 14\% decreasing in levels (62 peaks) was observed (Figure 57).

\section{UV-B acclimation and shock vs. shock only}

A greater number of significantly elevated metabolite levels were detected during the non-acclimation phase (UV-B shock only, 0 vs. $24 \mathrm{~h}$ ) compared to the UV-B acclimation phase ( 0 vs. 24 h). After 24 h of high UV-B, cultures not acclimatised 
previously to low levels of UV-B increased in significance (47\%) with a significant reduction in metabolite levels (negative $\log 2 \mathrm{FC}, 24$ vs. 48 h). Significant differences in cultures acclimatised to low UV-B followed by shock exposure did not change between the two phases (low UV-B 0 vs. 24 h, high UV-B 24 vs 48 h) demonstrating that cells can withstand the more damaging effects of higher intensity UV-B after acclimation phase. A greater level of significantly reduced abundances was observed during shock only high UV-B phase (24 vs. 48 h).

No metabolites relating to cyanobacterial metabolism were identified, with a match factor $>60 \%$, during the UV-B exposure experiment.

\subsubsection{UV-A acclimation and shock vs. shock only}

\subsubsection{Dry weight, pigment analysis and production of MAAs}

Similar dry weight patterns were observed for both acclimatised (Figure 58A) and non-acclimatised (Figure 58B) $C$. fritschii cultures during UV-A exposure with no significance comparing each time point (two-sample t-Test; acclimation and shock vs. shock only at $0,24,24.5,25.5$ and $48 \mathrm{~h}$ ). An increase in dry weight from $186.7 \mathrm{mg} \mathrm{L}$ ${ }^{1}$ to $196.7 \mathrm{mg} \mathrm{L}^{-1}$ was observed after $24 \mathrm{~h}$ of low UV-A with no significance $(p>0.05)$. A decrease during the first $24 \mathrm{~h}$ of non-acclimatisation (PAR only) was obsereved from $193.3 \mathrm{mg} \mathrm{L}^{-1}$ to $186.7 \mathrm{mg} \mathrm{L}^{-1}$ again, with no significance ( $\left.p>0.05\right)$. A higher significant increase in dry weight was observed between $24 \mathrm{~h}$ and $48 \mathrm{~h}$ of cultures acclimatised to low UV-A followed by shock with high UV-A ( 24 vs. $48 \mathrm{~h}, p<0.01$ ) compared to shock only cultures ( 24 vs. 48 h, $p<0.05$ ) but a slight increased growth rate (Figure 59) was measured during shock only exposure ( 24 to $48 \mathrm{~h}, \mu=0.03$ ) compared to acclimatised cultures ( 24 to $48 \mathrm{~h}, \mu=0.028$ ) with no significance calculated between the two (acclimation and shock vs. shock only; high UV-A, $p>0.05$ ). The growth rates (Figure 59) both showed significant increase comparing low UV-A $(\mu=0.002)$ vs. high UV-A $(\mu=0.028, p<0.01)$ and no UV-A $(\mu=-0.002)$ vs. high UV-A $(\mu=0.03$, $p<0.01)$ in both acclimatised and non-acclimatised cultures. 


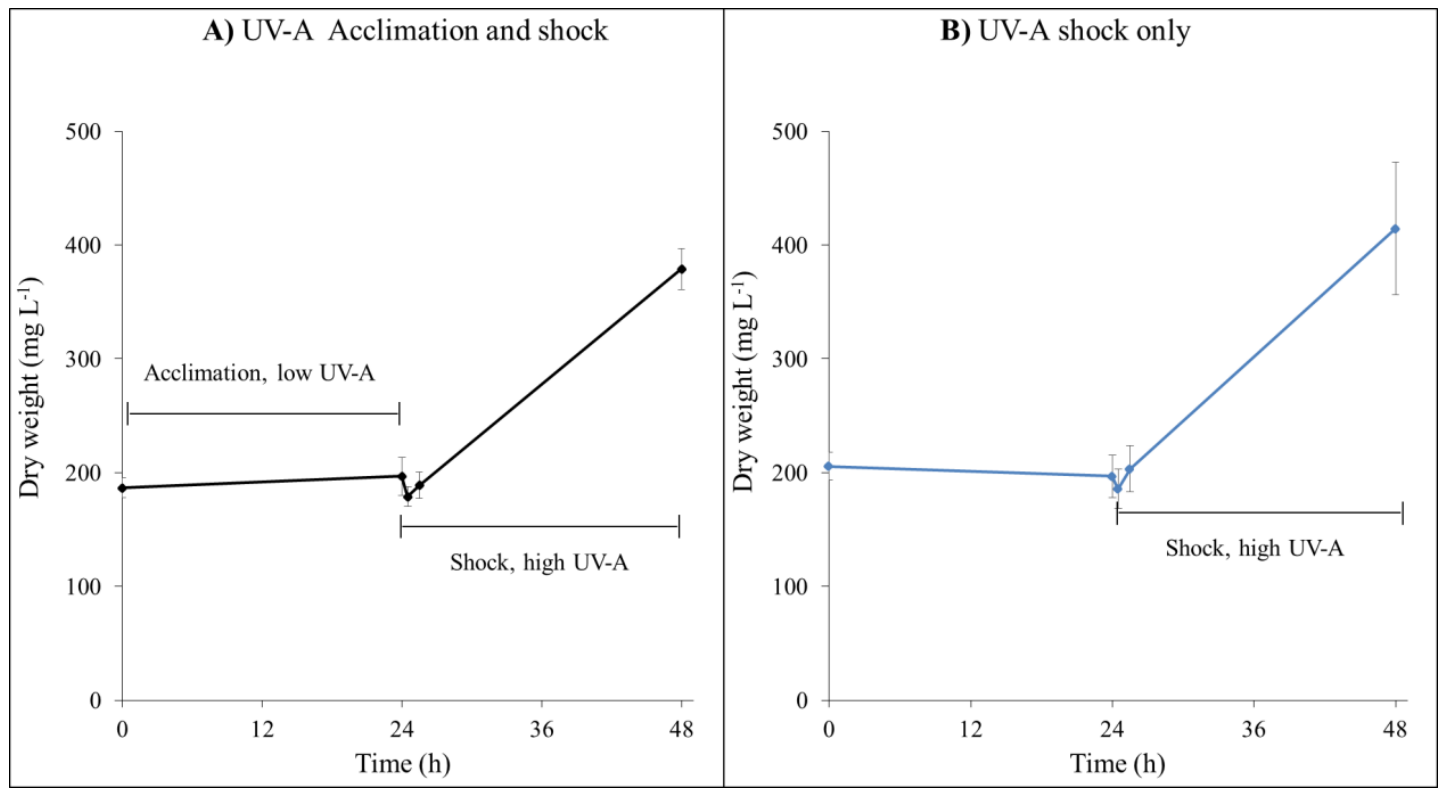

Figure 58: Dry weight analysis $\left(\mathrm{mg} \mathrm{L}^{-1}\right)$ of $C$. fritschii cultures during A) UV-A acclimation and shock and B) UV-A shock only. Data points are mean values of biological triplicates \pm SE. Statistical significance was measured using a two-sample t-Test, $*=0.05>p \geq 0.01$, $* *=0.01>p \geq 0.001$ and $* * *=p<0.001$

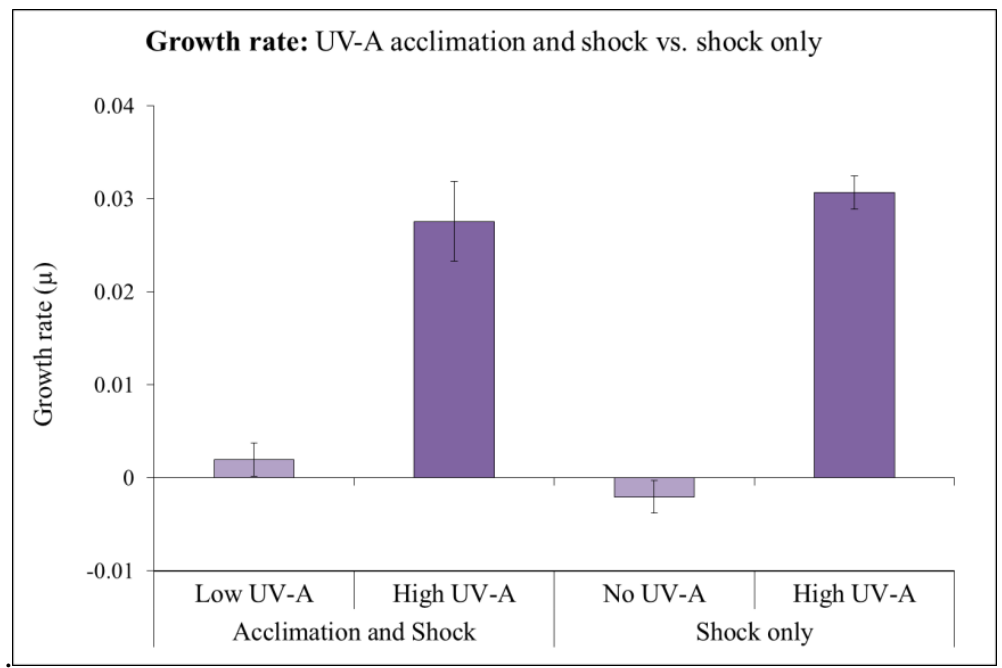

Figure 59: Growth rate $(\mu)$ of UV-A acclimation and shock vs. shock only of $C$. fritschii cultures. Low UV-A and no UV-A accounts for the growth rates between 0 and $24 \mathrm{~h}$ and high UV-A accounts for growth rate between 24 and 48 h. Data represents mean growth rates of biological triplicates \pm SE

Pigment concentration of $\operatorname{chl} a$ and carotenoids were measured during the time series. No significant difference in levels was observed during acclimation to low UV-A (0 vs. $24 \mathrm{~h}$ ) or during the shock, high UV-A, phase (24 vs. $48 \mathrm{~h}$ ) in both chl $a$ and carotenoid concentrations (Figure 60A). A significant increase in chl $a$ and carotenoid content was measured during the non-acclimation phase (Figure 60B; 0 vs. 24 h; chl 
$a, p<0.05$; carotenoid, $p<0.001$ ) as well as during the first $1.5 \mathrm{~h}$ of the shock phase (high UV-A: Carotenoids; 0 vs. 24.5 and 0 vs. 25.5, $p<0.01$ ).

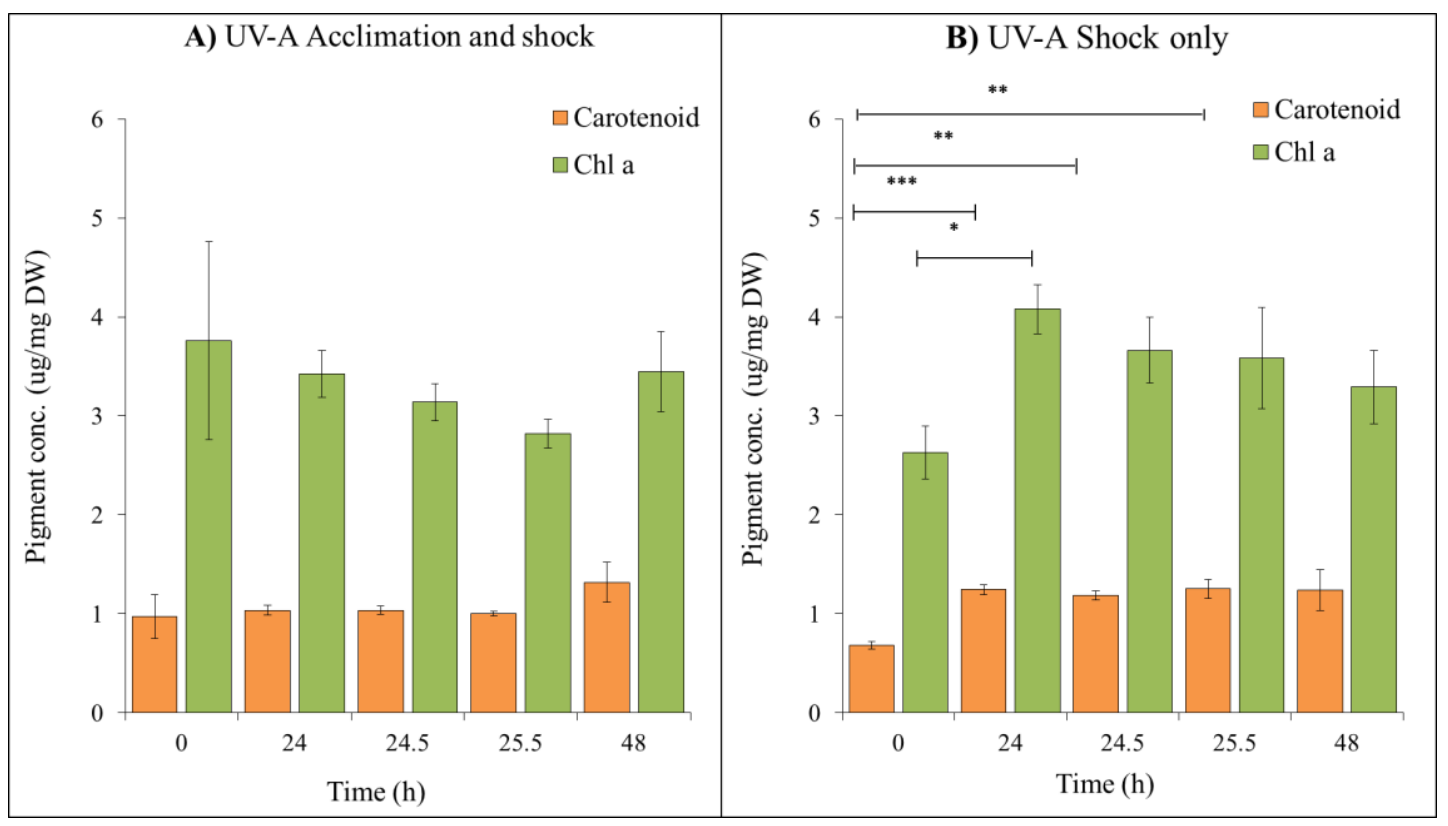

Figure 60: Pigment content ( $\mu \mathrm{g} / \mathrm{mg}$ dry weight) of $C$. fritschii cultures during A) UV-A acclimation and shock and B) UV-A shock only. Data points are mean values of biological triplicates \pm SE. Statistical significance was measured using a two-sample t-Test, $*=0.05>p \geq 0.01, * *=0.01>p \geq$ 0.001 and $* * *=p<0.001$.

Significant increases in shinorine content was measured after low UV-A ( 0 vs. 24 h, $p<0.01$ ), and during high UV-A exposure ( $p<0.05,0$ vs. 48 h). M-gly content also increased after $24 \mathrm{~h}$ of low UV-A followed by $24 \mathrm{~h}$ of high UV-A exposure ( 0 vs. 48 h, $p<0.05$ ) (Figure 61A). No MAAs were detected during PAR only exposure (shock only cultures, up to $24 \mathrm{~h}$ ), but significant increases in shinorine and m-gly was measured during high UV-A exposure (shinorine, 0 vs. $24.5,0$ vs. $25.5, p<0.001$; 0 vs. 48 h, $p<0.05$; m-gly, 0 vs. 48 h, $p<0.05$ ) (Figure 61B). Comparing between treatments (acclimation and shock vs. shock only), a significant increase in shinorine levels was calculated in acclimatised cultures after $24 \mathrm{~h}$ and $25.5 \mathrm{~h}$ acclimation and shock vs. shock only, $24 \mathrm{~h}$ and $25.5 \mathrm{~h} p<0.01$ ) 


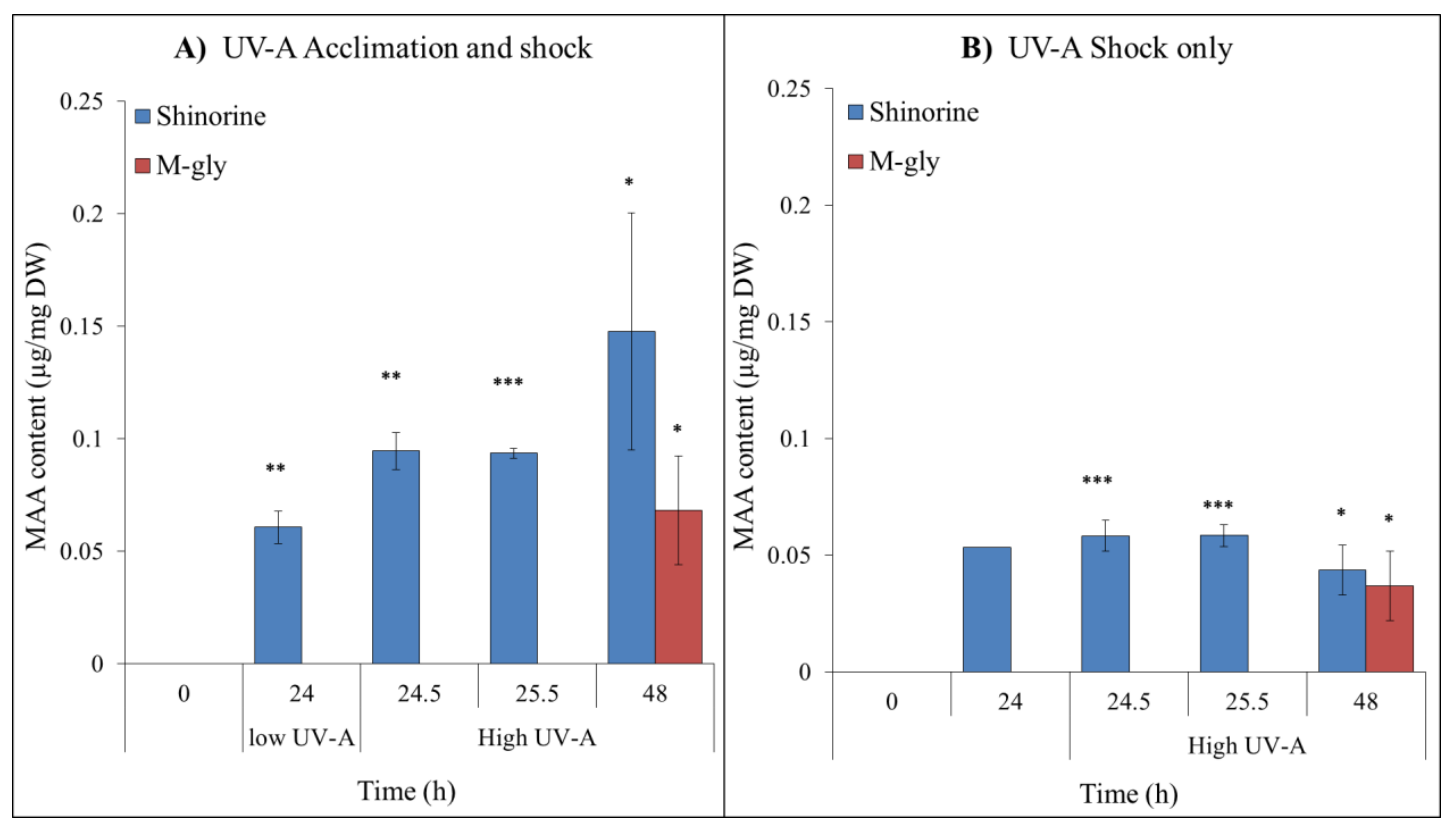

Figure 61: MAA content (pg/mg dry weight) of $C$. fritschii cultures during A) UV-A acclimation and shock and B) UV-A shock only. Data points are mean values \pm SE. Significant differences were measured using a two-sample t-Test comparing $0 \mathrm{~h}$ to each time point $(24,24.5,25.5$ and $48 \mathrm{~h}),{ }^{*}=$ $0.05>p \geq 0.01, * *=0.01>p \geq 0.001$ and $* * *=p<0.001$.

\subsubsection{Intracellular metabolite levels}

\section{UV-A shock only}

C. fritschii cultures were grown for $24 \mathrm{~h}$ under PAR only conditions (no UV-A) followed by $24 \mathrm{~h}$ of shock UV-A exposure (high UV-A). Samples for intracellular metabolite analysis were collected at 0,24 and $48 \mathrm{~h}$ for GC-MS analysis.

After analysis of the GC-MS data, a total of 332 peaks were detected. 127 of those were identified with a match factor $\geq 60 \%$ (level 2) and 205 remained unassigned where the acquired mass spectra had match factors $<60 \%$ (level 4 ).

Using a two-sample t-Test with equal variance comparing between time points $(0,24$ and $48 \mathrm{~h}$ ), 92 significant peaks ( $28 \%$ of total peaks, 332) were observed during the no UV-A phase ( 0 vs. 24 h). $15 \%$ of those increased significantly in levels (50 peaks with positive $\log 2 \mathrm{FC}$ ) and $13 \%$ decreased significantly (42 peaks with negative $\log 2 \mathrm{FC}$ ). 194 significant peaks were detected during the high UV-A phase (59\% of 332 total peaks; 24 vs. 48 h), with $16 \%$ elevating significantly in levels (52 peaks with positive $\log 2 \mathrm{FC}$ ) and 142 significantly reduced peaks ( $43 \%$ with negative $\log 2 \mathrm{FC}$, Figure 62). 


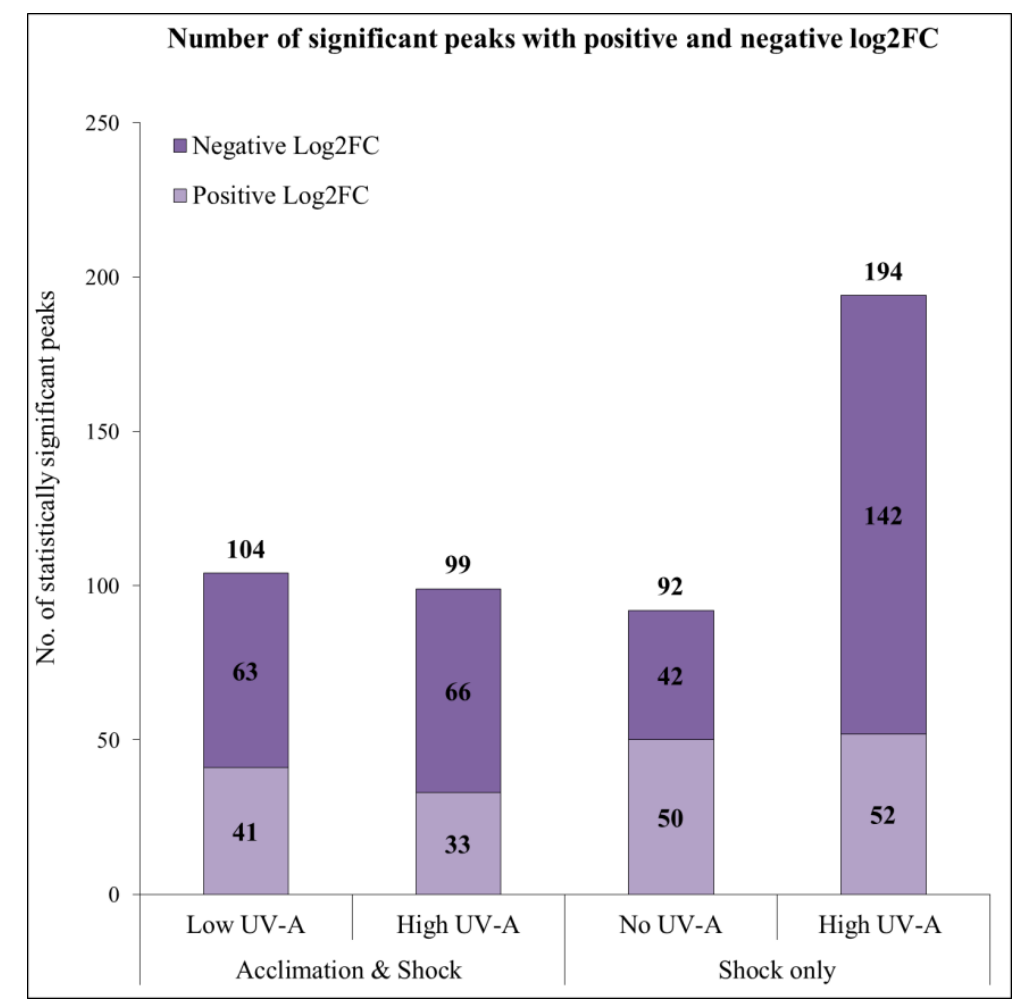

Figure 62: Total number of detected peaks that show significant differences $(p<0.05)$ during acclimation and shock (total peaks $=293$ ) and shock only (total peaks $=332$ ) comparing $0 \mathrm{vs.} 24 \mathrm{~h}$ (low UV-A or no UV-A) and 24 vs. 48 h (high UV-A) using a two-sample t-Test with equal variance. The number of significantly different peaks with positive and negative $\log 2$ FC comparing 0 vs. $24 \mathrm{~h}$ (low UV-A or no UV-A) and 24 vs. 48 h (high UV-A) is also presented.

33 identified peaks could be related back to pathways within cyanobacterial metabolism such as central carbon and nitrogen metabolism (Table S7).

The amino acids identified included; glu, val, pro, asp and ala. A significant decrease in glu ( $p<0.01,0$ to $24 \mathrm{~h}$ ) was observed after $24 \mathrm{~h}$ of PAR only. Val increased after 24 $\mathrm{h}$, followed by a decrease after $24 \mathrm{~h}$ of high UV-A exposure ( $24 \mathrm{vs.} 48$ h, $p<0.05$ ). Asp also increased after $24 \mathrm{~h}$ of PAR only ( 0 vs. $24 \mathrm{~h}, p<0.05)$ followed by a decrease after shock UV-A exposure ( 24 vs. 48 h, $p<0.05$ ), ala increased after 24 h of high UV-A exposure ( $p<0.05,24$ vs. 48 h). Pro increased after $24 \mathrm{~h}$ and decreased following high UV-A exposure with no significance $(p>0.05)$.

The 3 saturated fatty acids; mystiric, palmitic and stearic acid showed similar levels throughout the time series. All three increased after the first $24 \mathrm{~h}$ of PAR only (no UVA) with no significance followed by a decrease in levels after $24 \mathrm{~h}$ of shock (high UVA) showing significant difference in palmitic acid (24 vs. $48 \mathrm{~h}, p<0.05)$ and stearic 
acid (24 vs. 48 h, $p<0.05$ ) levels. Two mono-unsaturated fatty acids were detected; oleic acid showed an increase in levels after $24 \mathrm{~h}$ with a reduction after $24 \mathrm{~h}$ of high UV-A ( $p>0.05$ ), palmitoleic acid accumulation was observed after $24 \mathrm{~h}$ (no UV-A; 0 vs. $24 \mathrm{~h}, p<0.001)$ followed by a slight decrease in levels with no significance after high UV-A exposure.

Galactose, sucrose and raffinose levels accumulated after shock exposure to $24 \mathrm{~h}$ of high UV-A with significance observed in galactose levels (24 vs. 48 h, $p<0.001$ ). Glycerol levels decreased significantly after 24 and 48 h ( 0 vs. 24,0 vs. 48 h, $p<0.01)$ whereas glycerol-3-phosphate levels increased after $24 \mathrm{~h}$ of no UV-A ( 0 vs. $24 \mathrm{~h}$, $p<0.05)$ and after $24 \mathrm{~h}$ of shock UV-A (0 vs. $48 \mathrm{~h}, p<0.05)$.

Lactate levels increased significantly after the first $24 \mathrm{~h}(0$ vs. $24 \mathrm{~h}, p<0.05)$ with a decrease in levels after shock UV-A (no significance, $p>0.05$ ).

\section{UV-A acclimation and shock}

293 peaks were detected with 112 identified with a chemical name and class (level 2) with 181 left unassigned (level 4). 104 of those peaks showed significance comparing 0 and $24 \mathrm{~h}$ of low UV-A (36\% of total detected, 293). 14\% of those (41 peaks) increased significantly (41 peaks with positive $\log 2 \mathrm{FC}$ ) whilst $22 \%$ decreased significantly in levels (63 peaks with negative $\log 2 \mathrm{FC}$ ). 99 significant peaks were detected during high UV-A comparing 24 and 48 h (34\% of 293 total detected). 11\% of those had elevated levels by $48 \mathrm{~h}$ (33 peaks with positive $\log 2 \mathrm{FC}$ ) and $23 \%$ decreased significantly (66 peaks with negative $\log 2 \mathrm{FC}$, Figure 62).

27 of the identified peaks could be related back to central metabolism within cyanobacteria (Table S7). The three amino acid, glu, val and pro were identified. Glu and pro decreased in abundance during the time series with significance (glu, 0 vs. 48 h, $p<0.001$; pro, 24 vs. 48 h, $p<0.05)$. The fatty acids mystiric, palmitic and stearic acid showed no significant differences but levels decreased after $24 \mathrm{~h}$ of high UV-A. The sugars sucrose and trehalose increase in levels up to $48 \mathrm{~h}$ with no significance $(p>0.05)$. Phytol, a terpanoid involved in chlorophyll metabolism decreased in levels after $48 \mathrm{~h}$ ( $24 \mathrm{~h}$ of acclimation to low UV-A followed by $24 \mathrm{~h}$ of high UV-A) with significance ( $p<0.05,24$ vs. $48 \mathrm{~h})$. 


\section{UV-A acclimation and shock vs. shock only}

Despite very similar growth rates between UV-A acclimation and shock and shock only (Figure 59), metabolite profiles showed differences (Figure 62). A greater number of significantly elevated metabolites were observed in no UV-A phase compared to acclimation to low UV-A ( 0 vs. 24 h). By increasing the intensity of UVA, the increased total reduced metabolites showed significance after high UV-A with no previous acclimation compared to high UV-A with acclimation to low UV-A dose (24 vs. 48 h). Low UV-A has a lesser effect on C. fritschii as results indicate more similarity between low UV-A acclimation and no acclimation. Once the intensity of UV-A had increased, the cultures already acclimatised show lower significantly reduced levels whereas during high UV-A (without an acclimation phase), cultures show higher significant differences and an increase in reduced abundances as indicated by the negative $\log 2 \mathrm{FC}$ ( 24 vs. $48 \mathrm{~h}$ ).

24 common metabolites were identified within the two treatment groups (11 represented in Figure 63). Similar patterns were observed for mystic acid, stearic acid, palmitic acid, sucrose, trehalose, arabinose and glu. Differences in patterns were observed between pro, phytol, glycerol 3-phosphate raffinose and fructose. 

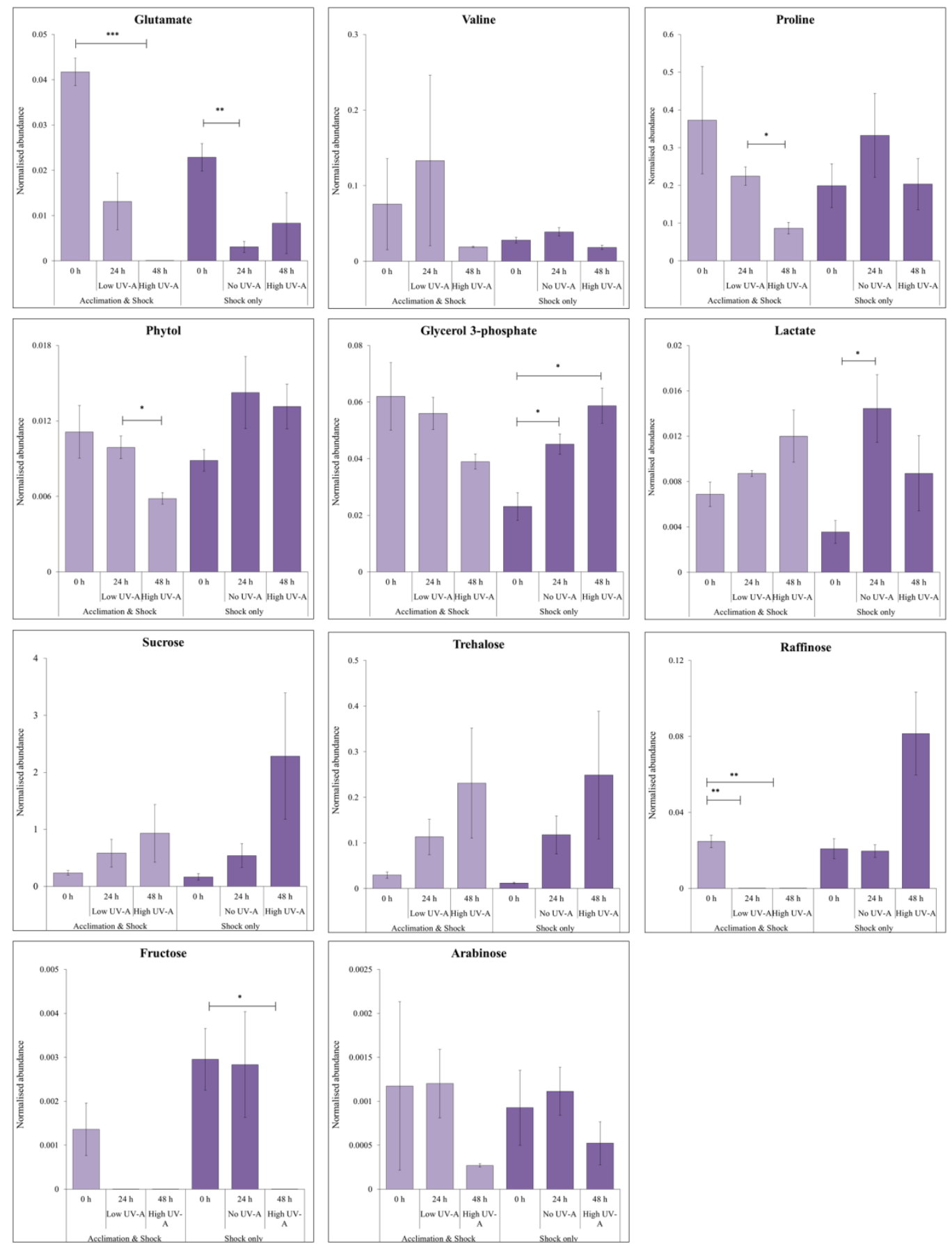

Figure 63: Common metabolites identified in both UV-A acclimation \& shock and shock only cultures. Data points are mean values of biological replicates \pm SE. Significant differences were measured using a two-sample t-Test, $*=0.05>p \geq 0.01, * *=0.01>p \geq 0.001$ and $* * *=p<0.001$. 


\subsection{Discussion}

The intensity of UV and the duration of the acclimation phase $(24 \mathrm{~h})$ were chosen from the dry weight curves from previous experiments (Chapter 3). After $24 \mathrm{~h}$ of UV-A (Figure 47) and UV-B (Error! Reference source not found.) exposure, the dry weight increased indicating the acclimation of cells to UV.

UV-A and UV-B affects cyanobacteria and other photosynthetic organisms in a variety of ways [24]. UV-B is more detrimental to cells as it causes direct and indirect damage whereas UV-A causes indirect damage by the production of ROS [27]. UV activates secondary metabolism resulting in the production of secondary metabolites such as MAAs and antioxidants that have potential industrial application [16,23].

C. fritschii is seen to withstand higher levels of UV-B irradiation after pre-treatment with low dose UV-B suggesting UV stress tolerance and adaptation. Growth was unaffected by UV-A acclimation and shock with increased dry weight at $48 \mathrm{~h}$ or both treatments. This is due to the less detrimental effect of UV-A on photosynthetic cells [27].

After low dose UV-B, an increase in pigment content (chl $a$ and carotenoid) was observed. This is the response of cells to ROS production and the antioxidant ability of carotenoids as previously described (Chapter 3). Both acclimatised and nonacclimatised cultures decreased in carotenoid content after $24 \mathrm{~h}$ of high levels of UVB. This could be due to the increased production of the sunscreen compounds MAAs which protect cells by absorbing excess radiation and dissipating as heat without the production of ROS [23]. Significant increases in pigment content in UV-A exposed cultures were observed during shock only again showing antioxidant abilities of carotenoids to ROS formation by indirect UV-A damage [138].

Increased MAAs were observed during UV-B low dose (acclimation) and high dose (shock). There were increased levels in acclimatised cultures with no significant differences to non-acclimatised cultures. Increased MAAs were also observed during low and high UV-A exposure, with again increased levels after acclimation. This again indicates the possible stress tolerance of $C$. fritschii to the increased levels of UV. Metabolism has already been rearranged for the production of MAAs thus preparing the cells for further UV protection. Further investigations are needed to assess if the 
concentration can be increased significantly with acclimatisation followed by shock exposure as a tool in industrial biotechnology to increase production of MAAs [16].

Overall a higher number of peaks with significantly reduced levels were detected during high UV-A and UV-B exposure only (negative log2FC). Although metabolite levels decreased overall in both UV-A and UV-B experiments, cultures acclimatised had a lower negative impact on metabolism. This also indicates the acclimation of cyanobacterial cultures to low dose UV, thus preparing cell metabolism for additional UV exposure as mentioned above.

During UV-B experiment, no biologically relevant metabolites relating to cyanobacterial metabolism were identified during the 48-h time-series. This could be due to a number of reasons including the identification process. For an identification to be made within these experiments, the mass spectrum of the sample must match with reference spectra at a threshold of $\geq 60 \%$ (level 2 identification [63]). Within these specific samples, no metabolites relating to cyanobacterial metabolites could be identified at this threshold. To overcome this in the future, standards of relevant metabolites should be run to confidently identify these metabolites (level 1 identification [63].

During the UV-A experiment, many identified metabolites had similar patterns as observed in the $48 \mathrm{~h} \mathrm{UV-A}$ experiment (Chapter 3). Fatty acids showed increases during low dose UV-A and PAR only, followed by a decrease in normalised abundance after $24 \mathrm{~h}$ of shock UV-A. This decrease could be due to the higher levels of UV-A. It is hypothesised that saturated fatty acids are desaturated during UV exposure to produce unsaturated fatty acids which have roles in UV protection. These unsaturated fatty acids possess double bonds which are throught to quench radicals produced by UV radiation. This is a controversial topic which needs further exploration [139]. Unsaturated fatty acids are also constituents of glycerolipid membranes and control their fluidity. Membrane fluidity is important for tolerance and acclimatisation to environmental stressors [140].

Raffinose and sucrose showed higher accumulation during UV-A shock, with only treatments with sucrose having a role in stress tolerance in cyanobacteria. Sucrose acts as a compatible solute (i.e, it does not interfere with cell metabolis) during salt stress $[99,127]$. Reduction in lactate levels during UV-A shock only could indicate the 
rearrangement of metabolism to prioritise mechanisms of secondary metabolite productions [120].

Glycerol 3-phosphate (G3P), involved in triacylglycerol (TAG) synthesis, has been seen to play a role in stress response in red algae [141]. Levels show accumulation in shock only cultures compared to decreasing levels in acclimatised cultures. TAG accumulation (via a decrease in G3P) can represent acclimation mechanisms [142].

Overall these results show that acclimatising cultures to lower dose UV (UV-A and UV-B) followed by high dose UV allows for better re-organisation of metabolism within $C$. fritschii cells as seen by the GC-MS results. Further investigations are needed to evaluate the changes at the metabolite levels during UV-B acclimation and shock.

\subsection{Conclusion}

In summary, similar dry weight was observed throughout the UV-A time-series showing $C$. fritschii's ability to adapt to UV-A stress over $48 \mathrm{~h}$. As this is a short-term study, evaluation of the long-term effects of UV-A at the metabolite level is needed as well as during a recovery period after UV-A exposure. An initial decrease in dry weight after $1.5 \mathrm{~h}$ of shock UV-B shows the initial direct damage to cells followed by further acclimation up to $24 \mathrm{~h}$ of shock exposure. UV-B induced production of higher levels of MAAs during shock exposure in both acclimatised and non-acclimatised cultures. A lesser negative impact on cell metabolism was observed after acclimation followed by shock UV-B exposure as seen in the GC-MS data indicating the stress tolerance and adaptation of $C$. fritschii. Further work is needed to identify these changes at the metabolite level in UV-B exposure acclimation and shock.

To my knowledge, this is the first UV acclimation followed by shock study to investigate the metabolite level changes in cyanobacteria, including $C$. fritschii. As mentioned previously, these experiments add to the knowledgebase of cyanobacterial adaptation to UV-A and UV-B exposure at the metabolite level as measured by GCMS. Manipulation of abiotic stress such as UV can further increase productivity of industrially relevant metabolites and improvement of UV tolerance in relevant species. 


\section{Chapter 5: Bioactivity Assessment of Chlorogloeopsis fritschii, PCC 6912 in in-vitro anti-infalmmatory and antibacterial assays}

\section{Aims}

- To cultivate $C$. fritschii under industrial conditions followed by sequential solvent extraction of biomass for bioactivity analysis.

- To develop an anti-inflammatory model using HaCaT cells.

- To use the developed method for the analysis of $C$. fritschii extracts.

- To test the effect of $C$. fritschii extracts on $\mathrm{HaCaT}$ proliferation using the real time iCELLigence $^{\mathrm{TM}}$ system.

- To test extracts for antibacterial activity against $S$. epidermis using the microdilution method.

- To analyse C. fritschii extracts using GC-MS to identify potentially active compounds. 


\section{Introduction}

Natural products from a variety of photosynthetic organisms such as; plants, macroalgae and microalgae have been widely investigated for their bioactive properties for uses in pharmaceuticals, cosmetics and nutraceuticals [143-146]. These provide sustainable alternatives to synthetic ingredients and are thought to be safer for human use and more environmentally friendly [147,148].

Cyanobacteria are promising sustainable alternatives to current sources of ingredients. They have simple growth needs, can utilise flue gas from industry as a source of $\mathrm{CO}_{2}$ and can be harvested throughout the year (non-seasonally). They have higher photosynthetic rates compared to plants and need less land area for cultivation. As prokaryotes they can be easily manipulated to form cell factories for higher production rates of valuable industrial compounds [1,16,149]. Cyanobacteria produce a vast array of bioactive metabolites with a variety of chemical structures. These compounds have potential applications as; anti-inflammatory, antimicrobial, photo-protective, moisturising and antioxidant ingredients for personal care products $[13,16,31]$.

The skin is the largest organ of the body. Functions include maintaining homeostasis in a variety of conditions and providing a protective barrier against the environment [150]. It is made up of 2 major layers; the epidermis and dermis comprising of a variety of cell types such as; keratinocytes and fibroblasts [150].

Inflammation is the response of the immune system to a variety of harmful stimuli involving immune cells, blood vessels and molecular mediators (such as cytokines and chemokines) [150]. Prolonged inflammation can result in many acute and chronic diseases such as cancer, diabetes, atherosclerosis, rheumatoid arthritis and Alzheimer's disease [151].

Inflammation of the skin occurs from infections and tissue damage from external factors such as microorganisms and UV exposure and from our own internal response to stress $[151,152]$. This can cause atopic dermatitis, acne vulgaris (acne), psoriasis and erythema (sunburn) to name a few. Increased ROS levels (from UV exposure) are associated with oxidative stress and protein oxidation. This can initiate inflammatory responses which are related to increased skin ageing [153,154]. 
Keratinocytes are the primary cell type and the major source of cytokines in the epidermis [155]. They can be used in in vitro skin models to evaluate proinflammation, anti-inflammation and proliferation [156]. HaCaT cells are a spontaneously immortalised keratinocyte cell line which have many similarities to primary keratinocytes [157]. They can be used as an alternative as they have a longer culture lifetime and do not vary between passages [156].

Cyanobacterial extracts and pure compounds have been evaluated for antiinflammatory, antioxidant and antibacterial properties. Examples include; Cphycocyanin from Spirulina platensis showed anti-inflammatory activity in lipopolysaccharide (LPS)-treated RAW 264.7 macrophages [158]. Nostoc commune and Spirulina platensis lipid extracts also showed anti-inflammatory activity in LPStreated RAW 264.7 macrophages by reducing pro-inflammatory cytokines (TNF- $\alpha$, IL-1 $\beta$ and IL-6) [159]. MeOH extracts from Nostoc commune Vauch showed antimicrobial activity against Bacillus subtilis, Staphylococcus aureus and Candida albicans using the agar diffusion assay [160].

This chapter aims to assess extracts from the cyanobacterium C. fritschii in in-vitro assays to evaluate anti-inflammatory, proliferation and antibacterial activity. To then use complementary GC-MS analysis of extracts for further elucidation of active ingredients with relevance to personal care products. 


\subsection{Material and methods}

\subsubsection{Anti-inflammatory model development}

\subsubsection{Seeding density experiment}

For the $\mathrm{HaCaT}$ cell seeding density experiment, a dilution series of cell concentrations was prepared (Table 7). Each dilution was added to a 24 microplate $\left(1 \mathrm{~mL}\right.$ well $\left.{ }^{-1}\right)$ and an E8 iCELLigence plate $\left(320 \mu \mathrm{L}\right.$ well $\left.{ }^{-1}\right)$ to determine the concentration of cells required for pro-/anti-inflammatory and proliferation assays (Section 1.8.3.3.1.).

\subsubsection{Pro-inflammatory dose response in HaCaT cells}

For pro-inflammatory dose response in HaCaT cells, TNF- $\alpha$ and IL-1 $\beta$ at final concentrations of 10, 5, $1 \mathrm{ng} \mathrm{mL}^{-1}$ and 1, 0.5, $0.1 \mathrm{ng} \mathrm{mL}^{-1}$ respectively (Table 8) were investigated using two concentrations of FBS in DMEM (10\% FBS; plate 1 and $1 \%$ FBS; plate 2) to determine the best mediator to induce the secretion of IL-6 and IL-8 in $\mathrm{HaCaT}$ cells (Section 1.8.3.3.3).

\subsubsection{Pro-inflammatory dose response of TNF- $\alpha$ (10\% FBS in DMEM)}

The procedure above (Section 5.2.1.2.) was repeated with an increased range of TNF$\alpha$ at final concentrations of; 10, 5, 1, 0.5, 0.1, 0.01 and $0 \mathrm{ng} \mathrm{mL}^{-1}$ (Table 9) in DMEM supplemented with $10 \%$ FBS only to determine the lowest concentration needed to stimulate IL-6 and IL-8 at sufficient quantities for anti-inflammatory experiments (Section 1.8.3.3.4.).

\subsubsection{Anti-inflammatory dose response of hydrocortisone in TNF- $\alpha$ induced inflammation in HaCaT cells.}

Anti-inflammatory dose response of hydrocortisone (HC) was investigated to determine the dose of $\mathrm{HC}$ required to inhibit TNF- $\alpha$ induced inflammation in $\mathrm{HaCaT}$ cells. Final concentrations of $10,1,0.1,0.01,0.001,0.0001$ and $0 \mu \mathrm{M}$ (Table 10) were

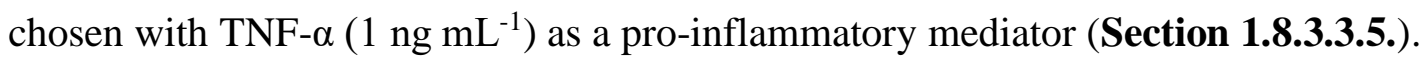

\subsubsection{Anti-inflammatory activity of pooled $C$. fritschii extracts in TNF-a induced inflammation of HaCaT cells}

Anti-inflammatory dose response of pooled C. fritschii UV-B and control extracts (Section 1.8.1.3. and 1.8.1.4) were investigated at final concentrations of $10,5,1,0.5$, 
$0.1,0.01$ and $0 \mu \mathrm{g} \mathrm{mL}^{-1}$ with $\mathrm{HC}$ as a control $(10 \mu \mathrm{M})$ and TNF- $\alpha\left(1 \mathrm{ng} \mathrm{mL}^{-1}\right)$ as a proinflammatory mediator (Section 1.8.3.3.6.).

\subsubsection{Anti-inflammatory activity of individual $C$. fritschii extracts in TNF-a induced inflammation of $\mathrm{HaCaT}$ cells}

Anti-inflammatory dose response of individual $C$. fritschii UV-B and control extracts (Section 1.8.1.3. and 1.8.1.4) were investigated at final concentrations of $1,0.5,0.1$ and $0 \mu \mathrm{g} \mathrm{mL}^{-1}$ with $\mathrm{HC}$ as a control $(10 \mu \mathrm{M})$ and TNF- $\alpha\left(1 \mathrm{ng} \mathrm{mL}^{-1}\right)$ as a proinflammatory mediator (Section 1.8.3.3.7.).

\subsubsection{Proliferation assay using iCELLigence real time analysis}

Cell proliferation assay was conducted using real time iCELLigence system. C.fritschii pooled control extracts $10,5,1,0.5,0.1$ and $0.05 \mu \mathrm{g} \mathrm{mL} \mathrm{L}^{-1}(\mathrm{n}=2)$ and control and UV-B pooled extracts at concentrations of $0.5,0.25$ and $0.1 \mu \mathrm{g} \mathrm{mL}-1(\mathrm{n}=2)$ were investigated (Section 1.8.4.)

\subsubsection{Anti-inflammatory activity of m-gly}

Anti-inflammatory dose response of m-gly (Section 1.8.2.) was investigated at final concentrations of $50,25,12.5,6.25,3.125$ and $0 \mu \mathrm{M}$ with $\mathrm{HC}$ as a control $(10 \mu \mathrm{M})$ and TNF- $\alpha\left(1 \mathrm{ng} \mathrm{mL}^{-1}\right)$ as a pro-inflammatory mediator (Section 1.8.3.3.8.).

\subsubsection{Antibacterial activity of $C$. frtischii extracts vs. S. epidermis}

Antibacterial activity of pooled and individual UV-B and control extracts were investigated against the test bacterium $S$. epidermis at concentrations ranging from 0.5 - 0.0156 $\mathrm{mg} \mathrm{mL}^{-1}$ and $0.5-0.03125 \mathrm{mg} \mathrm{mL}^{-1}$ respectively (Section 1.8.5.).

\subsubsection{GC-MS analysis of $C$. fritschii extracts}

$200 \mu \mathrm{L}$ of each extract was dried in a vacuum concentrator (Section 1.8.1.3.) and derivatised as in Section 1.3.3. GC-MS analysis was carried out using a PerkinElmer Turbo Mass ${ }^{\mathrm{TM}}$ (.raw files) with the same parameters and analysis as in Section 1.3.4. - 1.3.7. Statistical analysis was carried out on the identified biologically relevant metabolites only using the statistical analysis module in MetaboAnalyst. Statistical analysis was carried out using each column as a different extract $(n=3)$ and each row representing a metabolite (data type $=$ peak intensity table; data format $=$ samples in columns, unpaired). Missing data was replaced with half of the minimum integrated 
signal prior to analysis. Peaks were normalised to total sum of peaks, log transformed and mean centred prior to statistical analysis. PCA, a one-way ANOVA $(p<0.05)$ with post-hoc Tukey's honestly significant difference (HSD) and hierachial heat map clustering was used to evaluate the data. A two sample t-Test with equal variance was also used as a univariate statistical tool to evaluate data comparing each extract in Excel. 


\subsection{Results}

\subsubsection{Model development of an anti-inflammatory assay using HaCaT cells}

\subsubsection{Cell seeding density}

To begin model development, the optimum density of HaCaT cells was evaluated for use in anti-inflammatory assays. Cells were seeded at a variety of concentrations in a 24 well plate and growth was assessed using microscopic analysis after 24 and $48 \mathrm{~h}$ (Figure 64). Concentrations of $1 \times 10^{3}$ and $5 \times 10^{3}$ cells $\mathrm{mL}^{-1}$ were too dilute and the large spaces between cells prevented proliferation. Cells at a concentration of $1 \times 10^{5}$ cell $\mathrm{mL}^{-1}$ were able to reach a confluent monolayer after $24 \mathrm{~h}$ and therefore chosen for subsequent anti-inflammatory models. Inflammation can then be induced evenly across the monolayer of cells. 


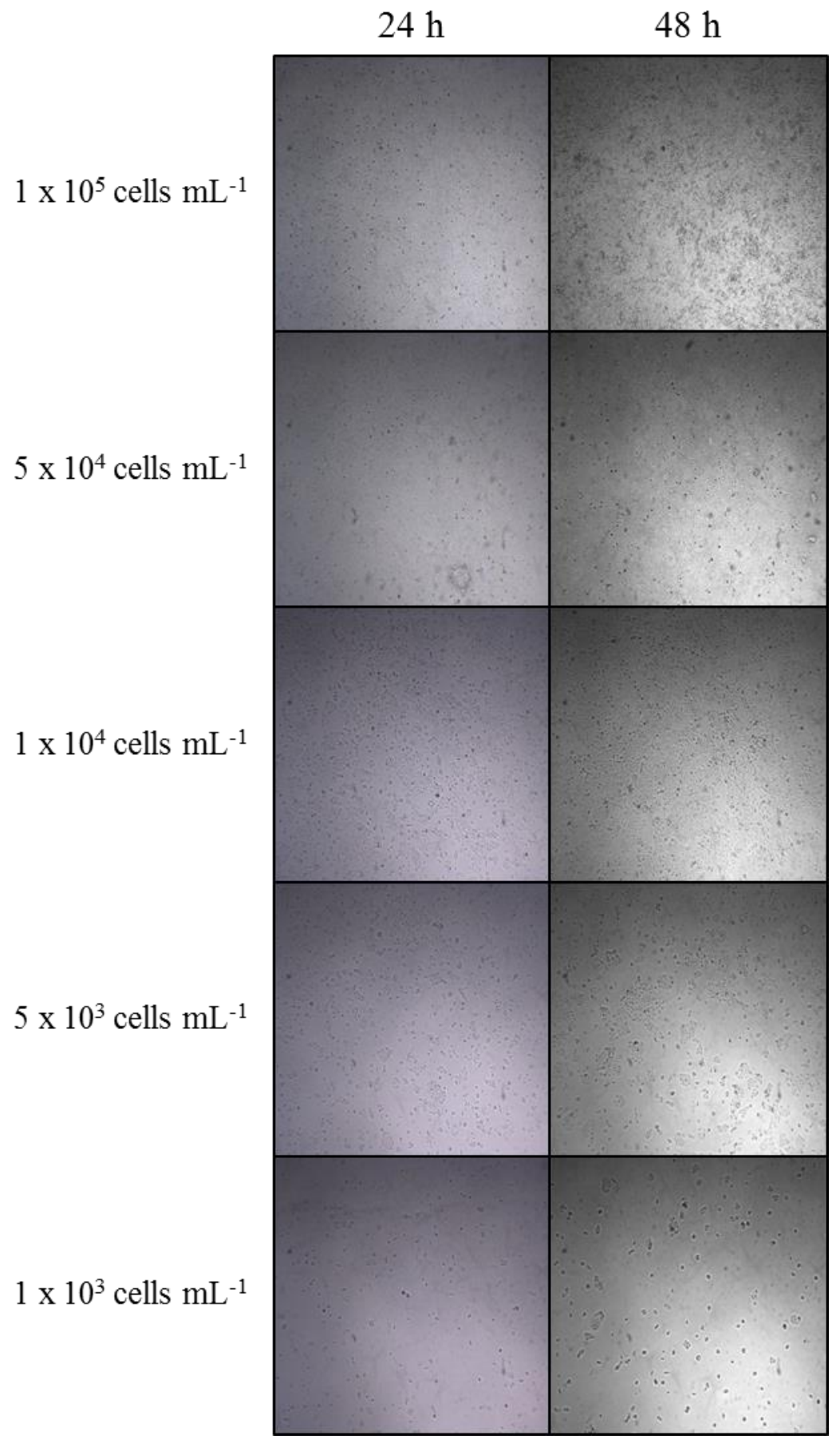

Figure 64: Microscope images (x4 lens) after $24 \mathrm{~h}$ and $48 \mathrm{~h}$ of growth of different cell densities of $\mathrm{HaCaT}$ cells in a 24 well plate. 


\subsubsection{Pro-inflammatory mediators}

Once the correct cell seeding density was chosen $\left(1 \times 10^{5}\right.$ cells $\left.\mathrm{mL}^{-1}\right)$ an appropriate pro-inflammatory mediator was needed to induce inflammation in monolayers of HaCaT cells. These are signalling molecules that are secreted from immune defence cells to promote inflammation [161] and include tumor necrosis factor alpha (TNF- $\alpha$ ) and interleukin-1 $\beta$ (IL-1 $\beta)$ [162].

HaCaT cells were firstly exposed to varying concentrations of TNF- $\alpha(1,5$ and $10 \mathrm{ng}$ $\left.\mathrm{mL}^{-1}\right)$ and IL-1 $\beta\left(0.1,0.5,1 \mathrm{ng} \mathrm{mL}^{-1}\right)$ for $24 \mathrm{~h}$ to stimulate the secretion of the proinflammatory cytokines; IL-6 and IL-8. This was tested using media supplemented with $1 \%$ fetal bovine serum (FBS) and 10\% FBS to see the effects of media supplementation on the induction of inflammation. The secretion of IL-6 and IL-8 was measured using an enzyme-linked immunosorbent assay (ELISA). Results showed an increased secretion of IL-6 and IL- 8 with TNF- $\alpha$ treatments (Figure 65A and B). Significance in IL-8 secretion was observed comparing vehicle control $\left(\mathrm{H}_{2} \mathrm{O} ; 0 \mathrm{ng} \mathrm{mL}\right.$ $\left.{ }^{1}\right)$ and $5 \mathrm{ng} \mathrm{mL}^{-1}(p<0.01)$ and $10 \mathrm{ng} \mathrm{mL}^{-1}(p<0.001)$ of TNF- $\alpha$ in 10\% FBS (Figure 65A) and at $10 \mathrm{ng} \mathrm{mL}^{-1}$ of TNF- $\alpha$ in $1 \%$ FBS (IL-6; $p<0.05$, IL-8; $p<0.05$ ) (Figure 65B). For both TNF- $\alpha$ and IL-1 $\beta$, an increased secretion in both IL-6 and IL- 8 was observed with 10\% FBS supplementation (Figure 65A and C). From these results, TNF- $\alpha$ was therefore chosen to stimulate the secretion of IL-6 and IL-8 in HaCaT cells cultured in $10 \%$ DMEM. 


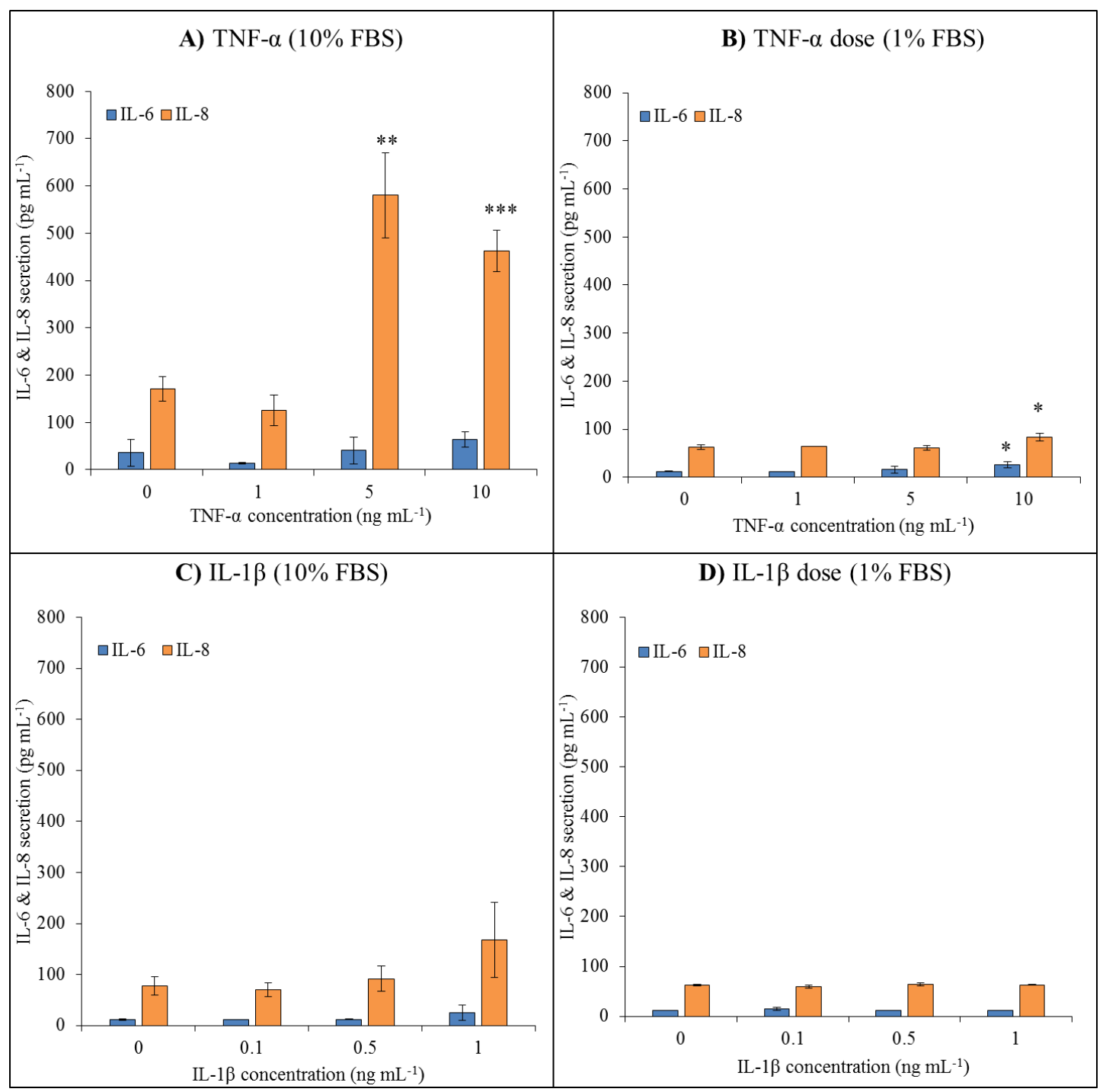

Figure 65: Evaluation of IL-6 and IL-8 secretion in A \& B) TNF- $\alpha$ and C \& D) IL- $1 \beta$ stimulated HaCaT cells. HaCaT's were exposed to concentrations of TNF- $\alpha\left(1,5\right.$ and $\left.10 \mathrm{ng} \mathrm{mL}^{-1}\right)$ and IL-1 $\beta(0.1,0.5$ and $\left.1 \mathrm{ng} \mathrm{mL}^{-1}\right)$ and vehicle control $\left(\mathrm{H}_{2} \mathrm{O}, 0 \mathrm{ng} \mathrm{mL}^{-1}\right)$ for $24 \mathrm{~h}$. Cells were cultured in A \& C) $10 \%$ FBS in DMEM and B \& D) $1 \%$ FBS in DMEM. Data represents mean values $(n=3) \pm$ standard deviation (SD). Significance was calculated comparing TNF- $\alpha$ or IL-1 $\beta$ concentrations to the vehicle control using a two-sample t-Test. $*=0.05>p \geq 0.01, * *=0.01>p \geq 0.001$ and $* * *=p<0.001$.

\subsubsection{TNF- $\alpha$ dose response}

A dose dependant response using varying concentrations of TNF- $\alpha$ in $10 \%$ FBS was evaluated for IL-6 and IL-8 cytokine secretion (Figure 66). Data was normalised to protein content to remove variations in cell growth. A significant increase in IL-6 secretion was observed after $24 \mathrm{~h}$ of treatment with $0.5(p<0.05), 5(p<0.001)$ and 10 $(p<0.05) \mathrm{ng} \mathrm{mL} \mathrm{mL}^{-1}$. Overall, a higher amount of IL-8 was secreted from HaCaT cells with significance at $0.5 \mathrm{ng} \mathrm{mL}^{-1}(p<0.01), 1,5$ and $10 \mathrm{ng} \mathrm{mL}^{-1}(p<0.001)$. 


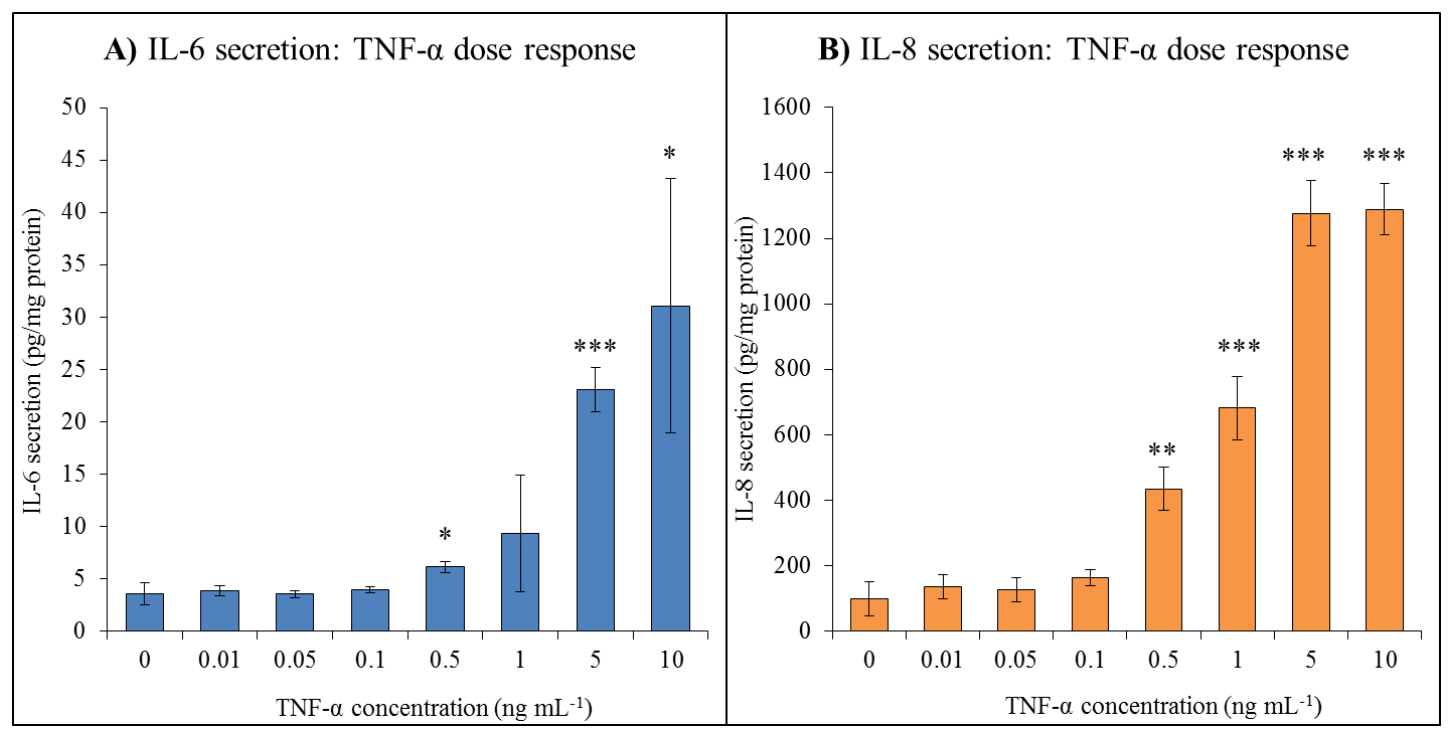

Figure 66: Evaluation of A) IL-6 and B) IL-8 secretion in TNF- $\alpha$ stimulated HaCaT cells. HaCaT's were exposed to varying concentrations of TNF- $\alpha\left(0.01-10 \mathrm{ng} \mathrm{mL}^{-1}\right)$ and vehicle control $\left(\mathrm{H}_{2} \mathrm{O}\right)$ for 24 h. Data represents mean values $(n=3) \pm S$. Significance was calculated comparing TNF- $\alpha$ concentrations and vehicle control using a two-sample t-Test. $*=0.05>p \geq 0.01, * *=0.01>p \geq 0.001$ and $* * *=p<0.001$.

\subsubsection{Hydrocortisone dose dependant anti-inflammatory activity}

Hydrocortisone (HC) was chosen as a positive control as a known anti-inflammatory agent. From the previous experiment $10 \mathrm{ng} \mathrm{mL}{ }^{-1}$ of TNF- $\alpha$ was chosen to ensure maximum induction of inflammation in the HaCaT cells. Dose dependant antiinflammatory activity was evaluated. Results showed reduced IL-6 and IL-8 secretions with higher HC concentrations (Figure 67) (IL-6; 0.1, 1 and $10 \mu \mathrm{M}, p<0.001$ and IL8; $0.1 \mu \mathrm{M} p<0.05,1 \mu \mathrm{M} p<0.01$ and $10 \mu \mathrm{M} p<0.001)$. HC at a concentration of $10 \mu \mathrm{M}$ was therefore chosen to ensure high anti-inflammatory response. 


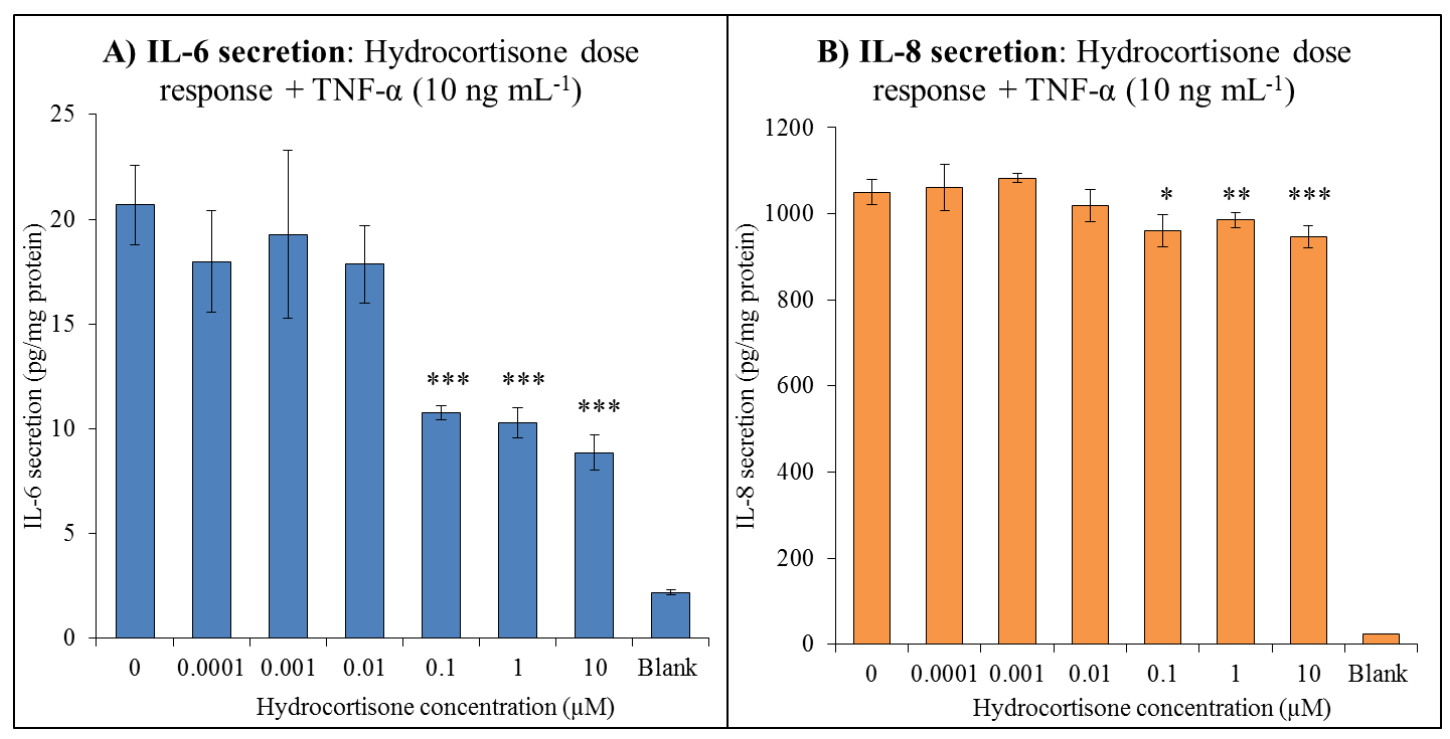

Figure 67: Hydrocortisone (HC) dose response of A) IL-6 and B) IL-8 secretion in TNF- $\alpha$ stimulated $\mathrm{HaCaT}$ cells. HaCaT's were exposed to varying concentrations of hydrocortisone $(10-0.0001 \mu \mathrm{M})$ or vehicle control (DMSO, $0 \mu \mathrm{M}$ ) for $24 \mathrm{~h}$ followed by incubation with TNF- $\alpha\left(10 \mathrm{ng} \mathrm{mL}^{-1}\right)$ and $\mathrm{HC}$ or vehicle control for a further $24 \mathrm{~h}$. Data represents mean values $(\mathrm{n}=3) \pm \mathrm{SD}$. Significance was calculated comparing $\mathrm{HC}$ vehicle control to $\mathrm{HC}$ doses using a two-sample t-Test* $=0.05>p \geq 0.01,{ }^{* *}=$ $0.01>p \geq 0.001$ and $* * *=p<0.001$.

Induction of HaCaT cells with TNF- $\alpha$ at a dose of $10 \mathrm{ng} \mathrm{mL}^{-1}$ induced higher levels of IL-8 secretions. A lower dose of $1 \mathrm{ng} \mathrm{mL}{ }^{-1}$ of TNF- $\alpha$ was therefore chosen for further evaluations of $C$. fritschii extracts to ensure activity can be quantified. Due to the secretion of IL-6 below detection limit of ELISA kit with $1 \mathrm{ng} \mathrm{mL} \mathrm{mL}^{-1}$ of TNF- $\alpha$, IL-8 only was investigated in further experiments.

Final workflow can be seen in Figure 68. 


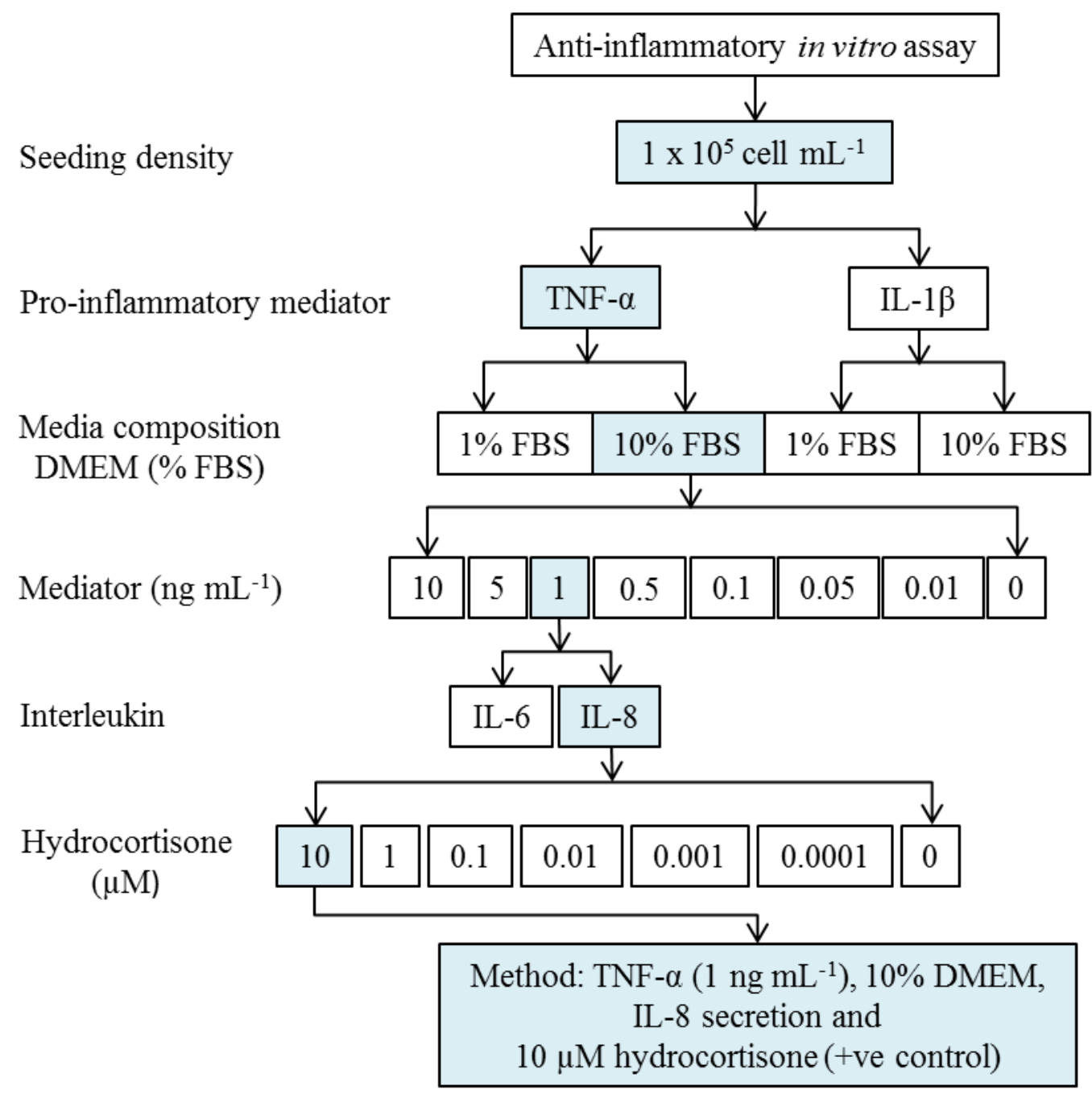

Figure 68: Anti-inflammatory method development workflow schematic.

\subsubsection{Analysis of $C$. fritschii extracts for anti-inflammatory activity}

C. fritschii metabolites were extracted sequentially with 4 solvents of differing polarity (Section 1.8.1). The hex, EtOAc, EtOH and $\mathrm{MeOH}$ extracts $\left(10 \mathrm{mg} \mathrm{mL}^{-1}\right.$ in DMSO) were pooled for assessment of anti-inflammatory activity using the developed model above (Figure 68).

A dose response of the pooled control and UV-B extracts was investigated by monitoring IL-8 secretion after incubation with extracts $\left(0.1-10 \mu \mathrm{g} \mathrm{mL}^{-1}\right)$ and TNF- $\alpha$

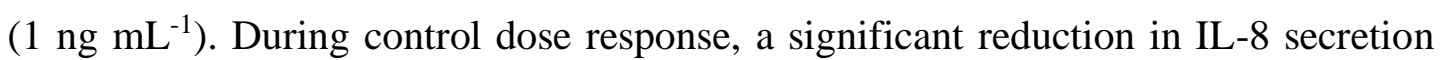
was observed after the addition of $0.1 \mu \mathrm{g} \mathrm{mL}^{-1}(p<0.01)$ and $1 \mu \mathrm{g} \mathrm{mL}^{-1}(p<0.01)$ of extract (Figure 69A). Similarly, UV-B exposed pooled extracts reduced IL-8 levels at concentrations of $0.1 \mu \mathrm{g} \mathrm{mL}^{-1}(p<0.05)$ and $0.5 \mu \mathrm{g} \mathrm{mL}^{-1}$ (UVB, $p<0.05$, Figure 69B). 


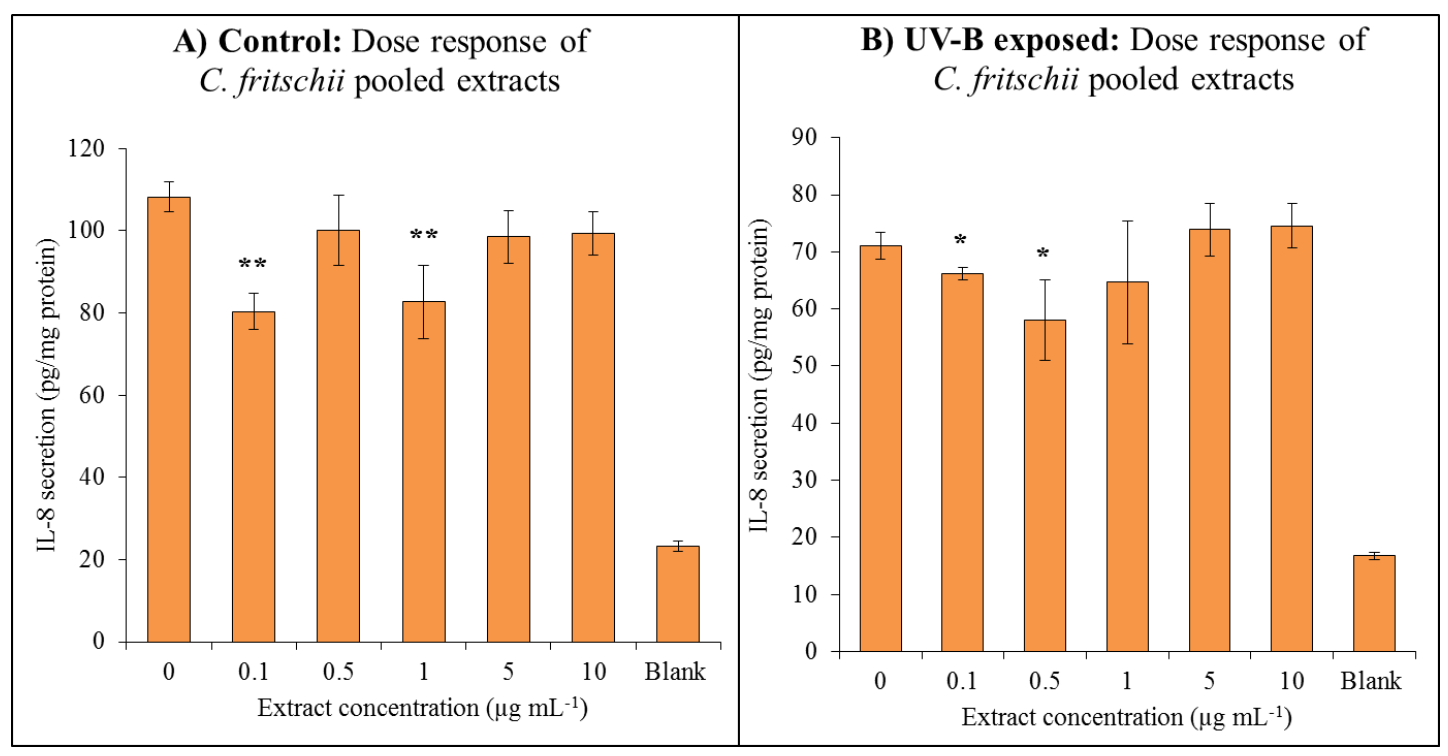

Figure 69: Dose response of A) control $C$. fritschii pooled extracts and B) UV-B exposed $C$. fritschii extracts on IL-8 secretion in TNF- $\alpha$ stimulated HaCaT cells. HaCaT's were exposed to extracts or vehicle control (DMSO) for $24 \mathrm{~h}$ followed by incubation with TNF- $\alpha(1 \mathrm{ng} \mathrm{mL}-1)$ and extracts for a further $24 \mathrm{~h}$. Data represents mean values $(n=3) \pm$ SD. Significance was calculated comparing extract vehicle control and extract doses using a two-sample t-Test. $*=0.05>p \geq 0.01, * *=0.01>p \geq 0.001$ and $* * *=p<0.001$.

In both experiments the highest concentrations (5 and $10 \mu \mathrm{g} \mathrm{mL}^{-1}$ ) show no significance (Figure 69). This could be due to the purity of the samples due to vast number of metabolites extracted from the $C$. fritschii cells that could inhibit any antiinflammatory activity of specific metabolites. There is no significant increase in IL-8 secretion which suggests that the pooled extracts do not induce inflammation in $\mathrm{HaCaT}$ cells.

After pooled extracts were assessed, the individual solvent extracts were analysed for activity at concentrations of $1,0.5$ and $0.1 \mu \mathrm{g} \mathrm{mL}-1$ compared to the positive control (HC, $10 \mu \mathrm{M}$ ) for both control and UV-B extracts. These low concentrations had the highest significance in IL-8 reduction from the pooled extract results (Figure 69). An extract with the highest response at the lowest concentration is most desirable when looking for new active ingredients as it decreases the risk of any adverse effects.

In the individual control extracts (Figure 70), EtOAc showed a significant reduction in IL-8 secretion at $0.1,0.5$ and $1 \mu \mathrm{g} \mathrm{mL}^{-1}(p<0.05)$. Other significant decreases were observed with $\operatorname{EtOH}\left(0.5 \mu \mathrm{g} \mathrm{mL}^{-1}, p<0.05\right)$ and $\mathrm{MeOH}\left(0.1 \mu \mathrm{g} \mathrm{mL}^{-1}, p<0.05\right)$ extracts. All extracts showed some reduction in IL-8 secretion and therefore anti-inflammatory 
activity except for hex. None of the individual extracts increased IL-8 secretion significantly above that of TNF- $\alpha$ control $\left(0 \mu \mathrm{g} \mathrm{mL}^{-1}\right)$ which suggest no proinflammatory activity against $\mathrm{HaCaT}$ cells.

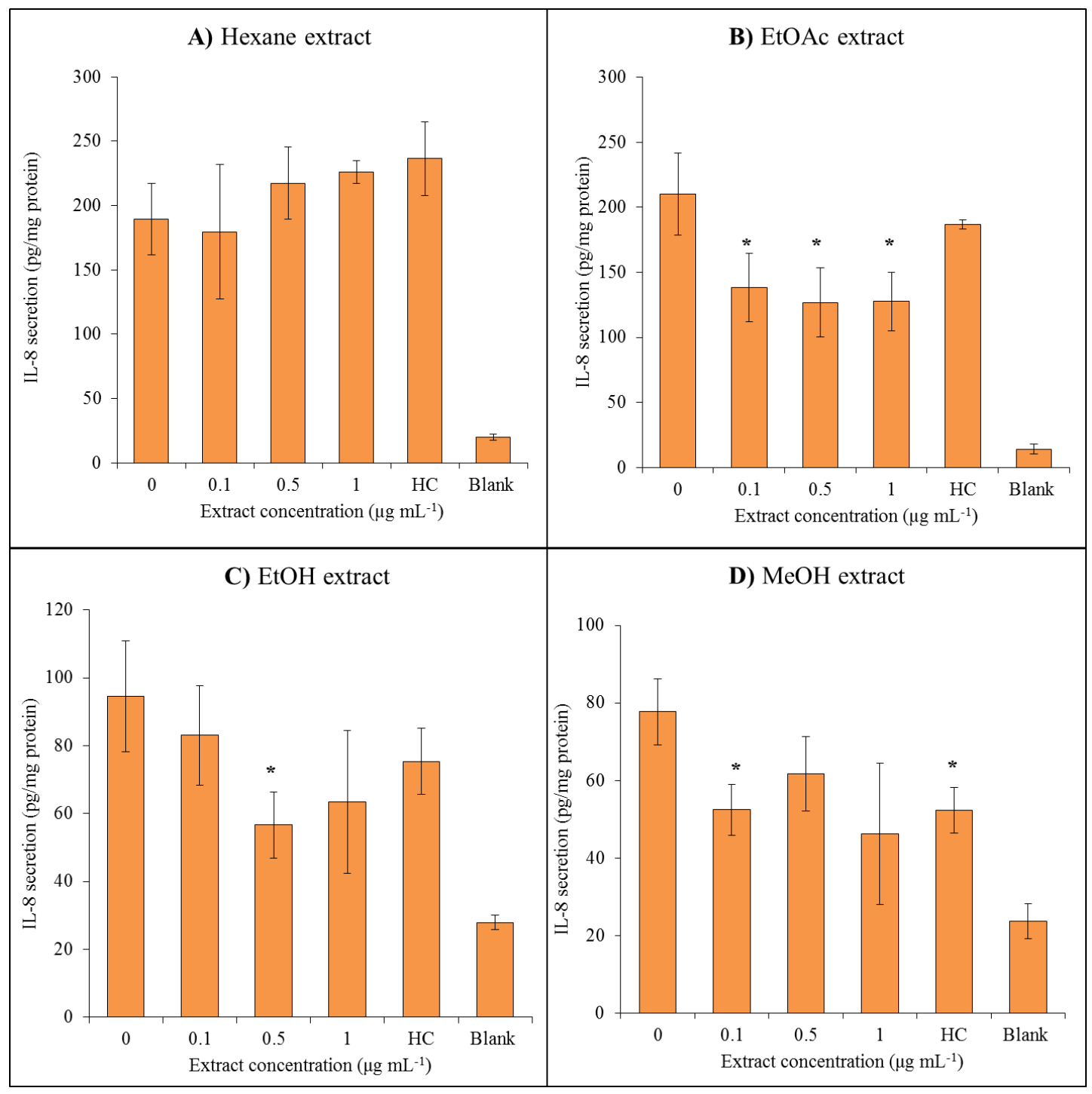

Figure 70: Effects of individual control C. fritschii extracts A) Hex, B) EtOAc, C) EtOH and D) $\mathrm{MeOH}$ on IL-8 secretion in TNF- $\alpha$ stimulated HaCaT cells. HaCaT's were exposed to extracts or vehicle control (DMSO) for $24 \mathrm{~h}$ followed by incubation with TNF- $\alpha\left(1 \mathrm{ng} \mathrm{mL}^{-1}\right)$ and extracts for a further 24 h. Data represents mean values $(n=3) \pm S D$. Significance was calculated comparing extract vehicle control and extract doses using a two-sample t-Test. $*=0.05>p \geq 0.01, * *=0.01>p \geq 0.001$ and $* * *=$ $p<0.001$. 
Individual UV-B extracts showed little differences in IL-8 secretion (Figure 71). TNF$\alpha$ has not been effective in increasing IL-8 levels. No significance between TNF- $\alpha$ and blank values were observed. Although this assay has been unsuccessful in analysing anti-inflammatory effect of the extracts it does show that these extracts are not proinflammatory. There was no significant increase in IL-8 secretion $(p>0.05)$.

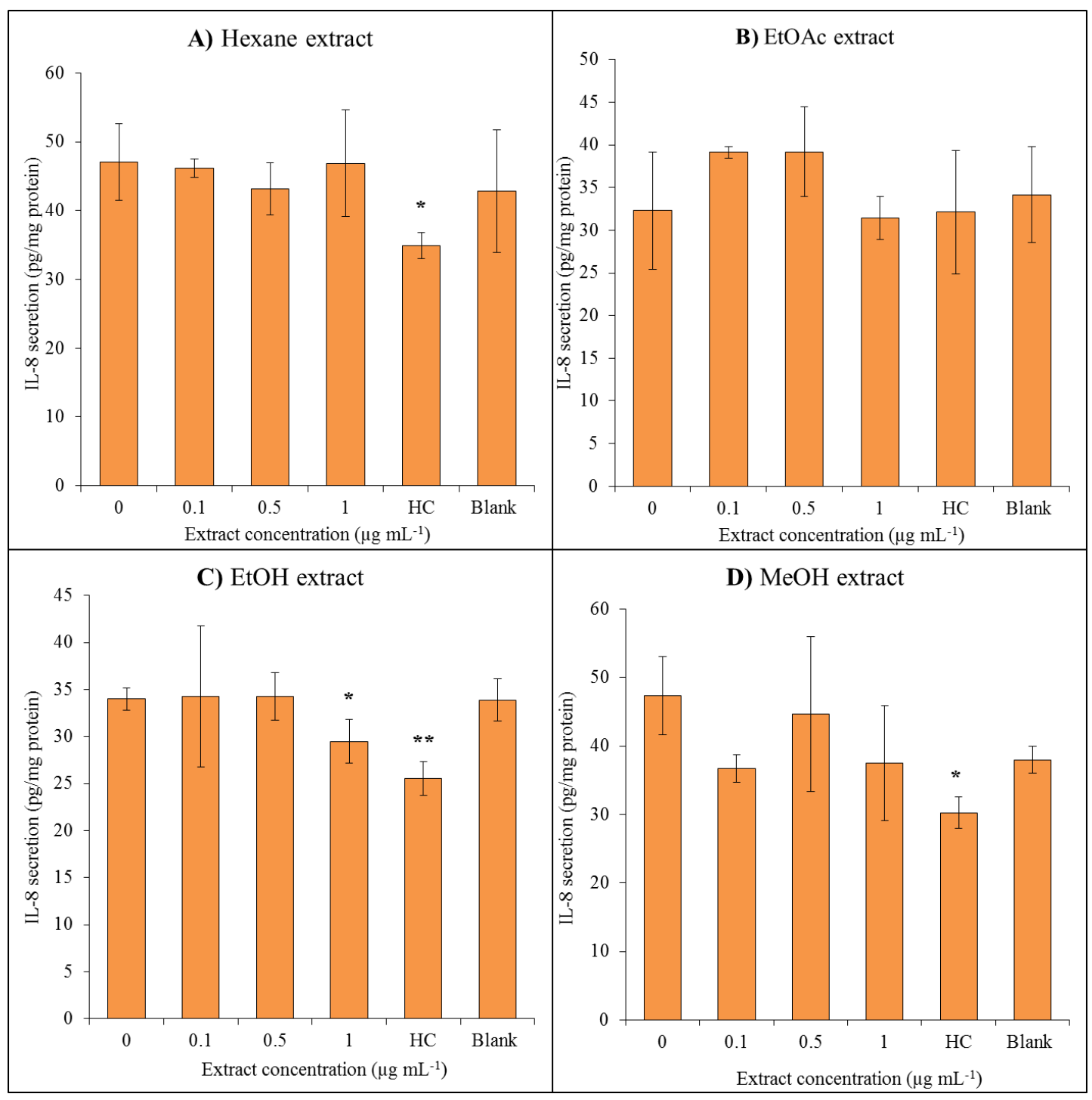

Figure 71: Effects of individual UV-B exposed $C$. fritschii extracts A) Hex, B) EtOAc, C) EtOH and D) $\mathrm{MeOH}$ on IL-8 secretion in TNF- $\alpha$ stimulated HaCaT cells. HaCaT's were exposed to extracts or vehicle control (DMSO) for $24 \mathrm{~h}$ followed by incubation with TNF- $\alpha\left(1 \mathrm{ng} \mathrm{mL}^{-1}\right)$ and extracts for a further $24 \mathrm{~h}$. Data represents mean values $(\mathrm{n}=3) \pm \mathrm{SD}$. Significance was calculated comparing extract vehicle control and extract doses using a two-sample t-Test. $*=0.05>p \geq 0.01, * *=0.01>p \geq 0.001$ and $* * *=p<0.001$. 


\subsubsection{Anti-inflammatory activity of mycosporine-glycine}

M-gly is one of two MAAs produced by $C$. fritschii (other being shinorie). Chapters 3 and 4 show that upregulation of MAA synthesis is seen during UV exposure. Although there is an increase in m-gly production during UV-B stress, the amount produced per gram of biomass is not enough to extract and use in anti-inflammatory models effectively. Due to this, heterologous expression in E. coli was used to produce a higher yield of m-gly to test in the developed model. This is an excellent prospect for industrial biotechnology by adding biosynthetic gene clusters to fast growing species such as E. coli to combat the low production yields of high value products.

A promising dose response (Figure 72) was observed with the lower concentrations reducing IL-8 secretion the most (no significance observed, $p>0.05$ ). IL-8 increased with the higher concentrations of m-gly $(25$ and $50 \mu \mathrm{M})$ which could be due to the purity of the extract. As the m-gly had a purity of $16 \%$ many different compounds could also be present within the extract which could increase inflammation as discussed above.

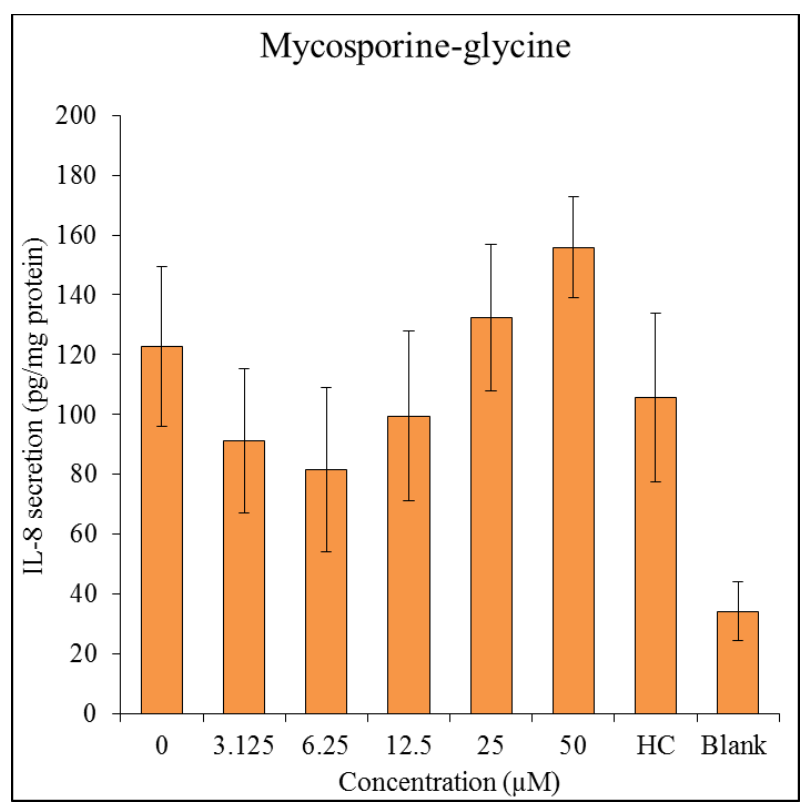

Figure 72: Effect of m-gly on IL-8 secretion in TNF- $\alpha$ stimulated HaCaT cells. HaCaT's were exposed to m-gly $(3.124-50 \mu \mathrm{M}), \mathrm{HC}(10 \mu \mathrm{M})$ or vehicle control $\left(\mathrm{H}_{2} \mathrm{O}\right)$ for $24 \mathrm{~h}$ followed by incubation with TNF- $\alpha\left(1 \mathrm{ng} \mathrm{mL}^{-1}\right)$ and $\mathrm{m}$-gly for a further $24 \mathrm{~h}$. Data represents mean values $(\mathrm{n}=3) \pm \mathrm{SD}$ 


\subsubsection{Effect of $C$. fritschii extracts on HaCaT proliferation}

For proliferation assessment, real-time analysis was conducted using the RTCA iCELLigence $^{\mathrm{TM}}$ system where cell proliferation can be monitored label-free [163]. This gives advantages over the traditional end-point labelled methods such as WST-1 and MTT assays [164,165]. Increased cell index (CI) shows the increased level of cell adherence to the bottom of the wells. To begin, Seeding density was optimised by seeding HaCaT cells at varying concentrations and measuring cell index over 48 h. 5 x $10^{4}$ cells $\mathrm{mL}^{-1}$ were confluent by $24 \mathrm{~h}$ whereas $1 \times 10^{4}$ cells $\mathrm{mL}^{-1}$ showed cell proliferation up to $48 \mathrm{~h}$ (Figure 73). A cell concentration between these two values, at $2 \times 10^{4}$ cells $\mathrm{mL}^{-1}$, was therefore chosen as cells need to be in a growth phase during treatment to evaluate how extracts affect cell proliferation (Figure 73).

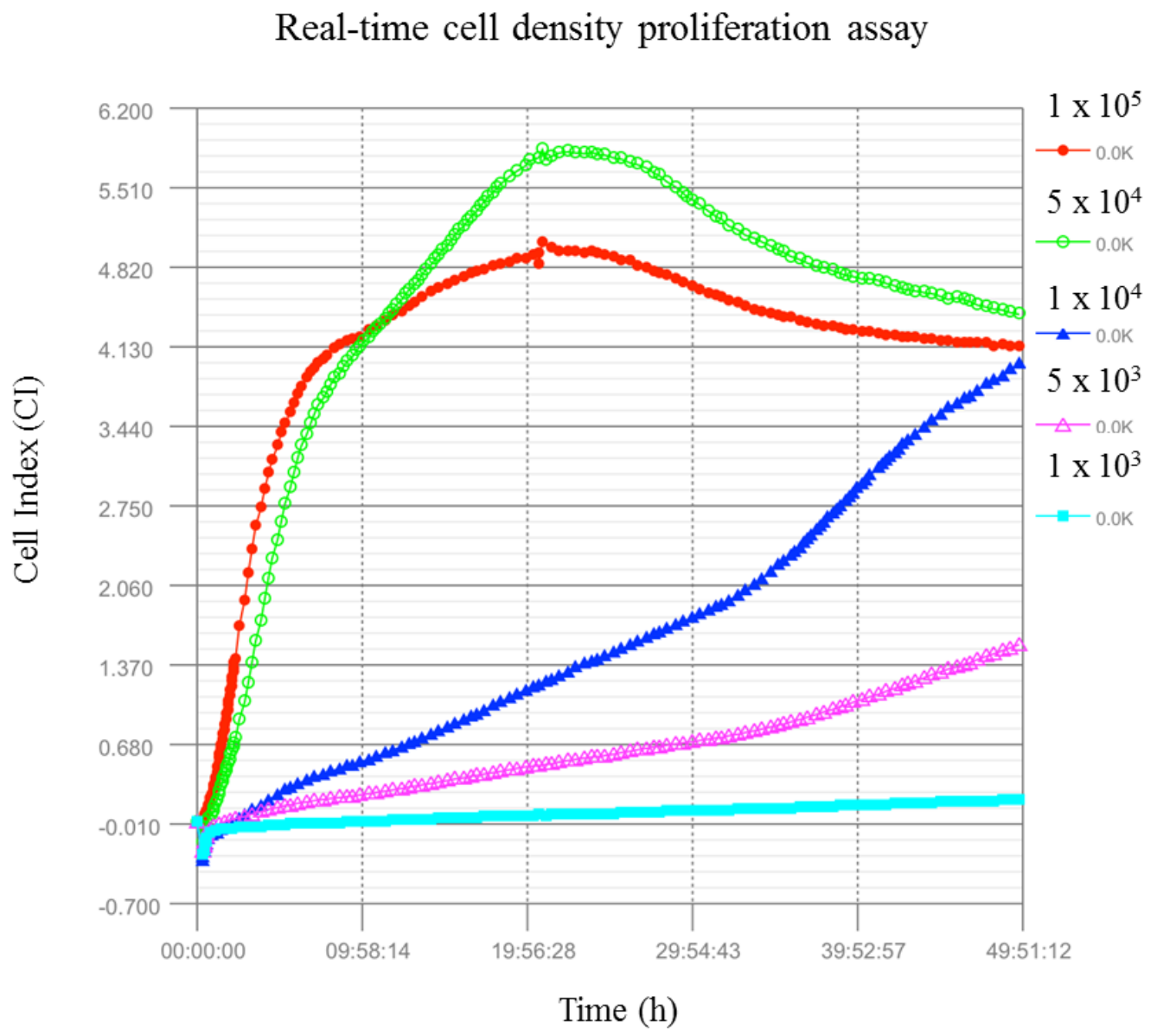

Figure 73: iCELLigence data of cell density experiment over approx. $48 \mathrm{~h}$. Data points are mean values of 2 replicates $(n=2)$. 
Proliferation experiments were then conducted using pooled control and UV-B extracts. After dosing HaCaT cells with pooled extracts for $24 \mathrm{~h}$, a slight increase in proliferation was observed with the control extracts. A second experiment was conducted with an increase in proliferation time to $72 \mathrm{~h}$. Both control and UV-B exposed extracts were evaluated at concentrations of $0.1,0.25$ and $0.5 \mu \mathrm{g} \mathrm{mL} \mathrm{m}^{-1}$ with little difference compared to the extract free control.

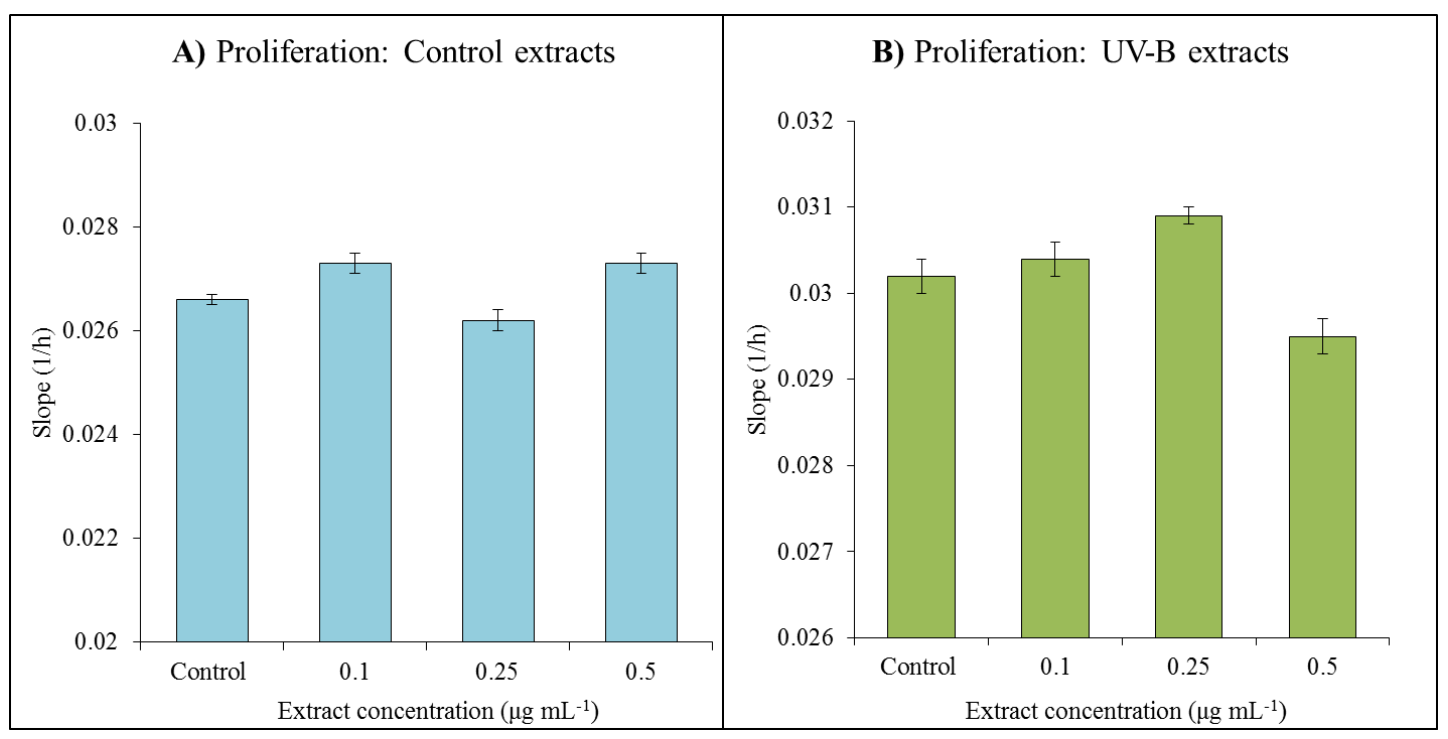

Figure 74: Rate of $\mathrm{HaCaT}$ cell proliferation with A) control extract treatment and B) UV-B extract treatment. Gradient of the slope between 24 and $72 \mathrm{~h}$ was investigated. Data represents mean values of 2 replicates $(n=2)$.

\subsubsection{Analysis of $C$. fritschii extracts for antibacterial activity}

S. epidermis is the most common bacterium found on the human skin. It is grampositive and aerobic. This test bacterium was chosen for initial screening of antibacterial activity of $C$. fritschii extracts. This was assessed using the microdilution method. The end point OD at $590 \mathrm{~nm}$ was measured after $24 \mathrm{~h}$ of incubation of extracts with bacterial culture.

An initial investigation of pooled extracts from both control and UV-B exposed cultures were assessed for antibacterial activity. One replicate was used due to the limited amount of extract available. Comparing DMSO controls with the $C$. fritschii extracts, a decrease in growth is observed (Figure 75). UV-B samples are seen to have lower end point OD readings than the control growth extracts. This could relate to antibacterial activity of extracts but further investigations are requires due to limited replication $(\mathrm{n}=1)$. 


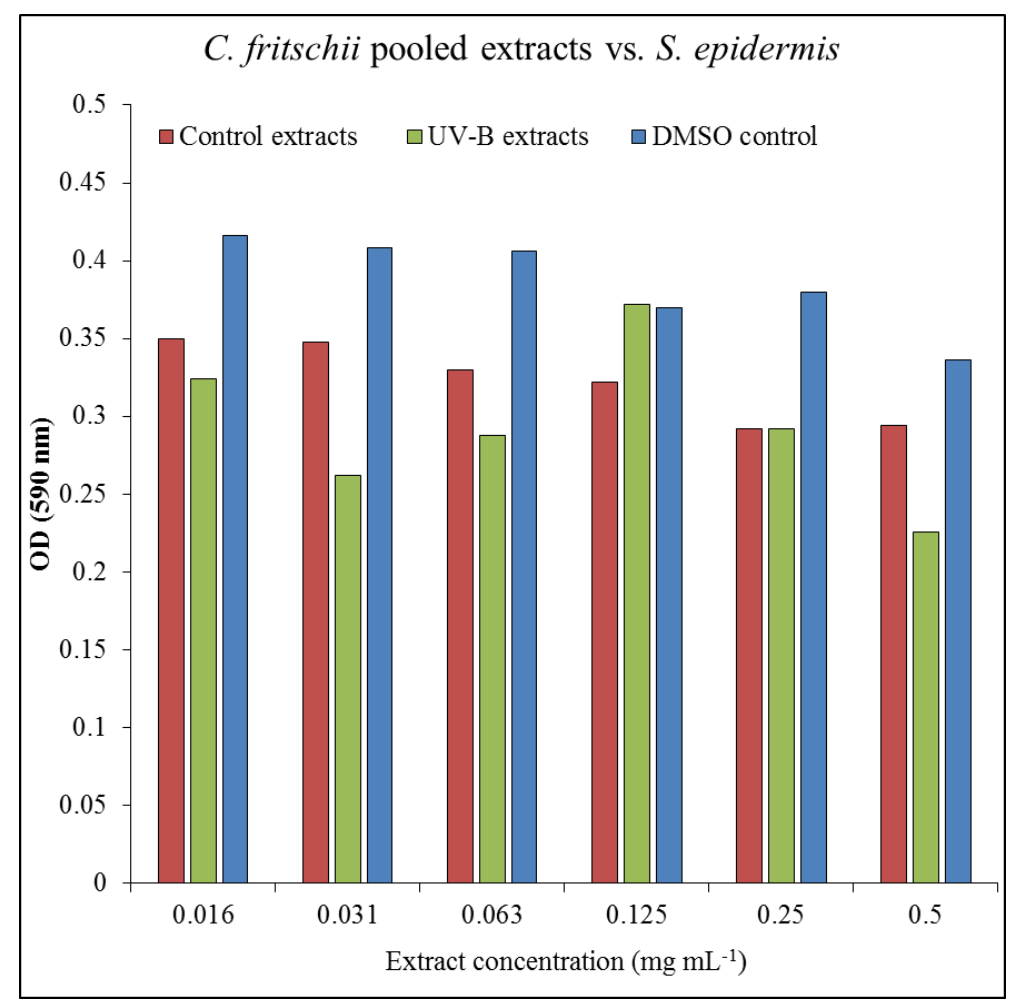

Figure 75: Antibacterial activity of pooled $C$. fritschii control and UV-B extracts at concentrations of $0.5,0.25,0.125,0.063,0.031$ and $0.016 \mathrm{mg} \mathrm{mL}^{-1}$ vs. S. epidermis. DMSO was used as a vehicle control. Data represents OD values from one reading $(\mathrm{n}=1)$.

This result was investigated further to assess the individual control and UV-B extracts (hex, EtOAc, EtOH and $\mathrm{MeOH}$ ) antibacterial properties against S. epidermis. These results are from two replicates again due to the limited amount of sample available.

The control hex and EtOAc extracts showed a similar range of OD end point readings to the DMSO vehicle control (Figure 76A and B). The EtOH fraction decreased the growth of $S$. epidermis the most at concentrations of 0.5 and $0.25 \mathrm{mg} \mathrm{mL}^{-1}$ (Figure 76C). $\mathrm{MeOH}$ extracts also decreased the growth of S. epidermis at a concentration of $0.25 \mathrm{mg} \mathrm{mL}^{-1}$ compared to the DMSO vehicle control but to a lower extrent than EtOH (Figure 76D). 


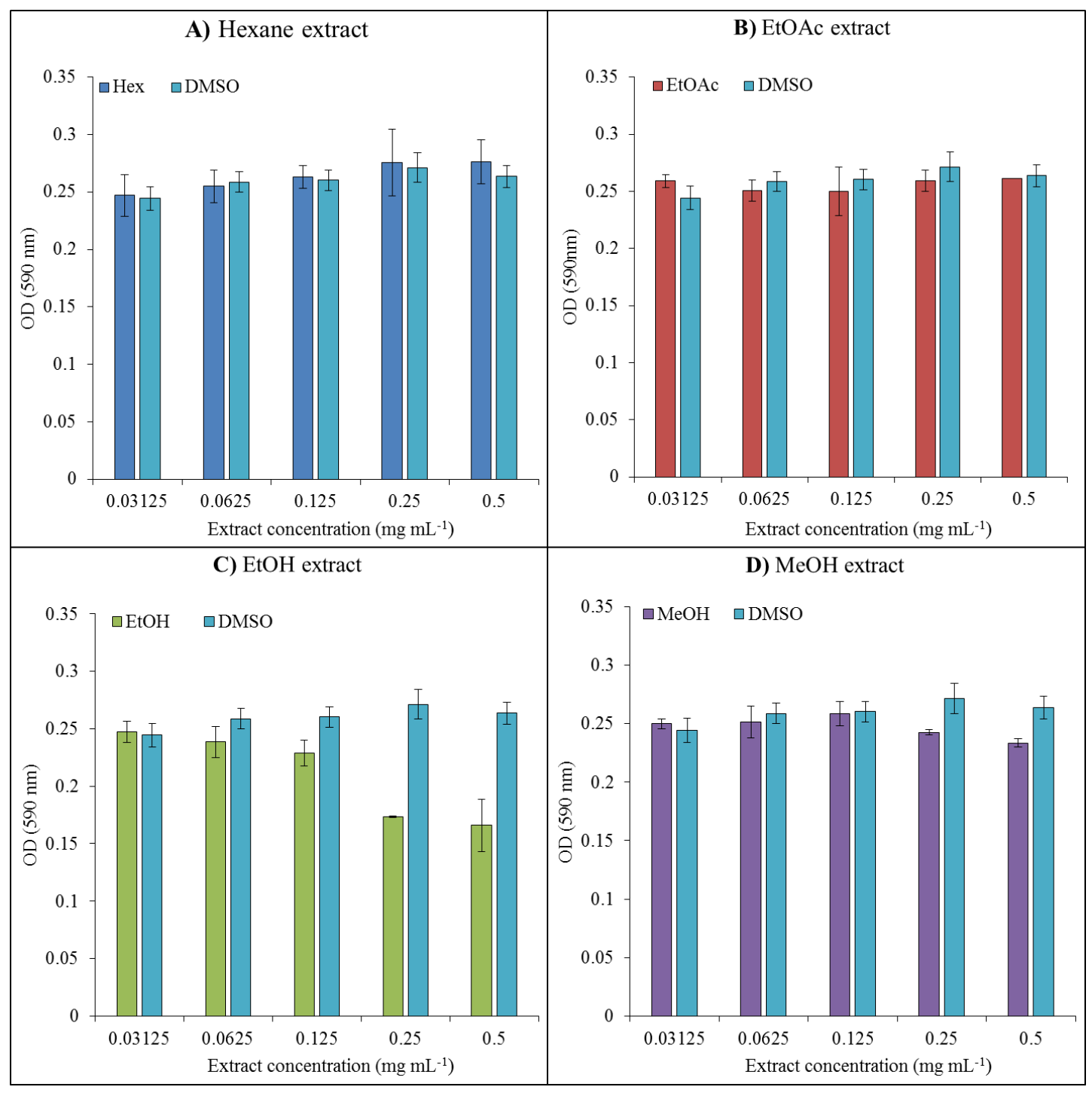

Figure 76: Antibacterial activity of $C$. fritschii control extracts: A) Hex, B) EtOAc, C) EtOH and D) $\mathrm{MeOH}$ at concentrations of $0.03125,0.0625,0.125,0.25$ and $0.5 \mathrm{mg} \mathrm{mL}^{-1}$ with DMSO as a vehicle control against $S$. epidermis using the microdilution method. Values are mean \pm range of two replicates $(n=2)$.

After exposing S. epidermis to the extracts from UV-B exposed C. fritschii cultures, EtOAc and MeOH extracts (Figure 77B and D) decreased the growth of the bacterium the most at the lowest concentration of $0.25 \mathrm{mg} \mathrm{mL}^{-1}$. The hexane extract (Figure 77A) showed no difference between the DMSO control whereas EtOH extract (Figure 77C) showed a decrease in end point OD at a concentration of $0.125 \mathrm{mg} \mathrm{mL}^{-1}$. 


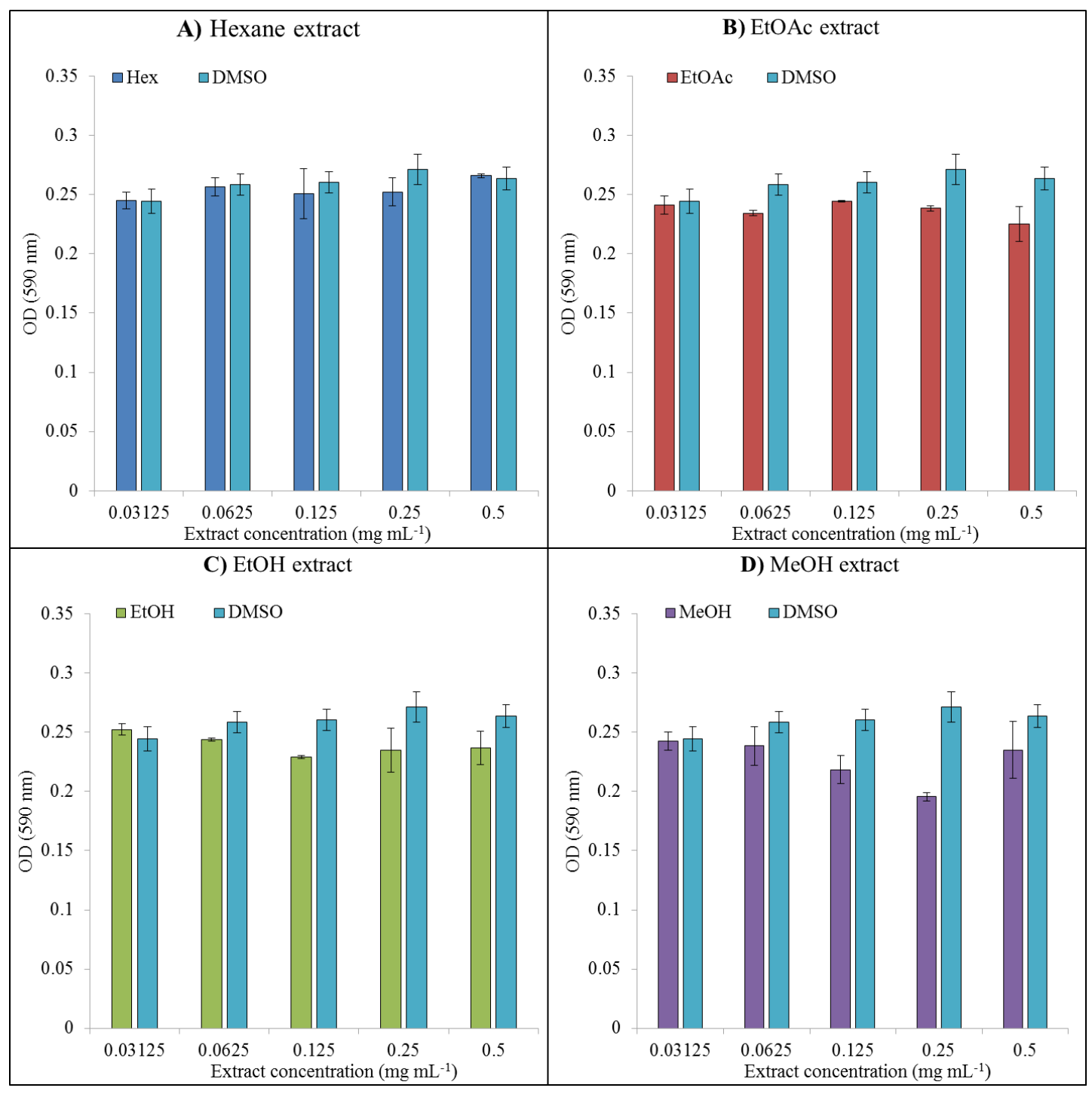

Figure 77: Antibacterial activity of $C$. fritschii UV-B extracts: A) Hex, B) EtOAc, C) EtOH and D) $\mathrm{MeOH}$ at concentrations of $0.03125,0.0625,0.125,0.25$ and $0.5 \mathrm{mg} \mathrm{mL}^{-1}$ with DMSO as a vehicle control against S. epidermis using the microdilution method. Values are mean \pm range of two replicates $(\mathrm{n}=2)$.

From the results obtained above, the extracts with the lowest OD end-point compared to the DMSO control were analysed again to see if the results were repeatable (Figure 78). Control EtOH and $\mathrm{MeOH}$ (Figure 78A) as well as UV-B EtOAc, EtOH and $\mathrm{MeOH}$ (Figure 78B) were investigated at concentrations of $0.0625-0.25 \mathrm{mg} \mathrm{mL}^{-1}$. A $50 \%$ reduction in bacterial growth was measured with control $\mathrm{EtOH}$ and $\mathrm{MeOH}$ extracts at $0.25 \mathrm{mg} \mathrm{mL}^{-1}$ as well as at $0.125 \mathrm{mg} \mathrm{mL}^{-1}$. A decrease in bacterial growth was also observed with UV-B EtOH extracts at $0.25 \mathrm{mg} \mathrm{mL}^{-1}$ and $0.125 \mathrm{mg} \mathrm{mL}^{-1}$ and $\mathrm{MeOH}$ extracts at 0.0625 and $0.125 \mathrm{mg} \mathrm{mL}^{-1}$. 


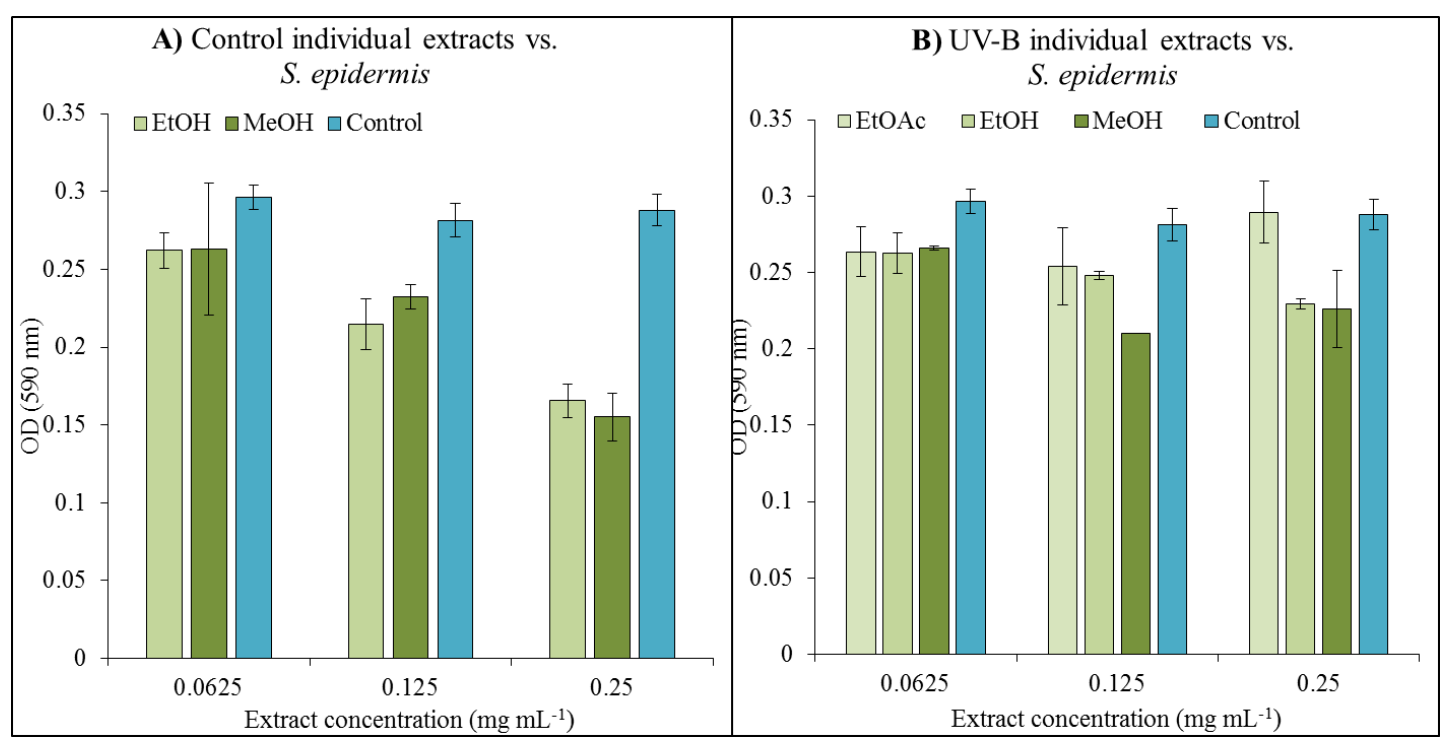

Figure 78: Antibacterial activity of A) control (EtOH and $\mathrm{MeOH})$ and $\mathrm{B}) \mathrm{UV}-\mathrm{B}(\mathrm{EtOAc}, \mathrm{EtOH}$ and $\mathrm{MeOH})$. fritschii extracts at concentrations of $0.0625,0.125$ and $0.25 \mathrm{mg} \mathrm{mL}^{-1}$ against $S$. epidermis with DMSO as a vehicle control. Data represents mean \pm range of two replicates $(n=2)$.

Overall, potential antibacterial activity against S. epidermis was observed with the EtOH and $\mathrm{MeOH}$ extracts as measured by an end-point OD microdilution method. Due to the nature of the highly pigmented extracts another type of assay would need to be used to reinforce this data. A non-colourimetric assay such as the disc diffusion or colony counting method could be investigated to complement this work [166]. More extract replicates are needed for statistical analysis of potential activity.

\subsubsection{GC-MS analysis of $C$. fritschii extracts}

Sequential solvent extraction was used to extract a variety of metabolites from $C$. fritschii biomass for bioactivity analysis. These extracts were also analysed using GCMS to assess metabolites for use in industrial biotechnology. Control extracts were evaluated only.

A total of 146 peaks were identified (level 2, match factor $\geq 60 \%$ ) across the four extraction solvents after conservative peak alignment in SpectConnect (control extracts only). Identified peaks were classified according to their chemical class including; acids, alcohols, amino acids, aldehyde, ether and ketones, aromatics, fatty acids/alcohols, heterocycles, hydrocarbons, N-compounds, S-compounds, sterols, sugar (and derivatives) and other. A greater number of peaks were detected and identified from the EtOAc extract (100) with the lowest peak identification from the hexane extract (74, Figure 79). EtOAc extracted higher numbers of acids, aromatics, 
fatty acids, heterocycles and sterols. A higher number of sugars (and derivatives) were identified in the $\mathrm{MeOH}$ extract.

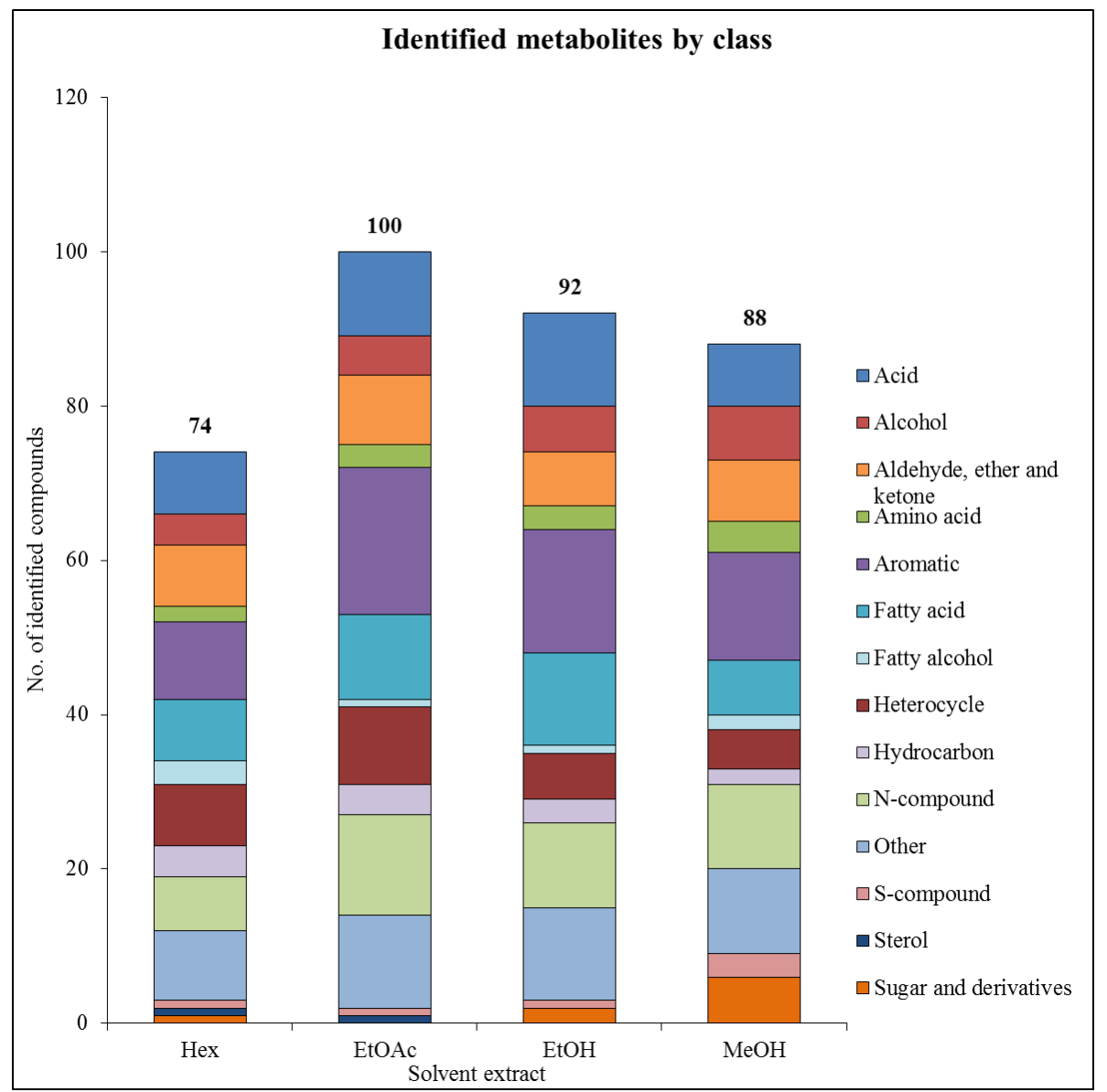

Figure 79: Number of identified peaks extracted from $C$. fritschii biomass by chemical class using hexane, EtOAc, EtOH and $\mathrm{MeOH}$.

Overall, 46 metabolites relating to cyanobacterial metabolism were identified in the extracts (Table 26). A PCA 2D-scores plot was used to visualise the variation between solvent extracts and revealed a total variation of $64.9 \%$ across two principle components $(\mathrm{PC} 1=46.6 \%$ and $\mathrm{PC} 2=17.9 \%$, Figure 80A $)$. Clear separation is observed between MeOH extract and Hex, EtOAc and EtOH.

One-way ANOVA revealed 16 metabolites with overall significance. Post-hoc analysis using Tukey's honestly significant difference (HSD) revealed that $\mathrm{MeOH}$ extract contributed mostly to the significance (Table S8) as seen in the PCA 2D score plot and the Hierarchial heat map (Figure 80B). 
A)

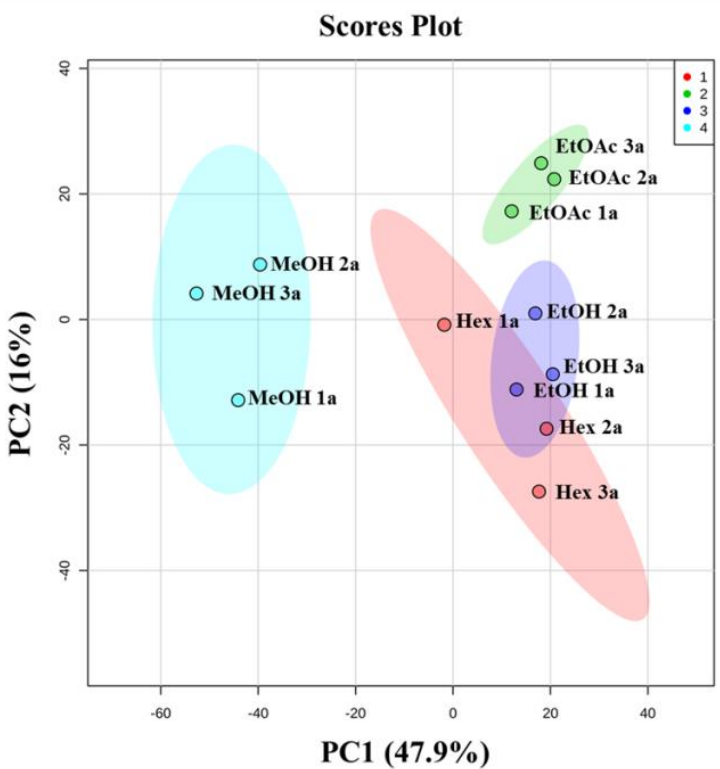

B)

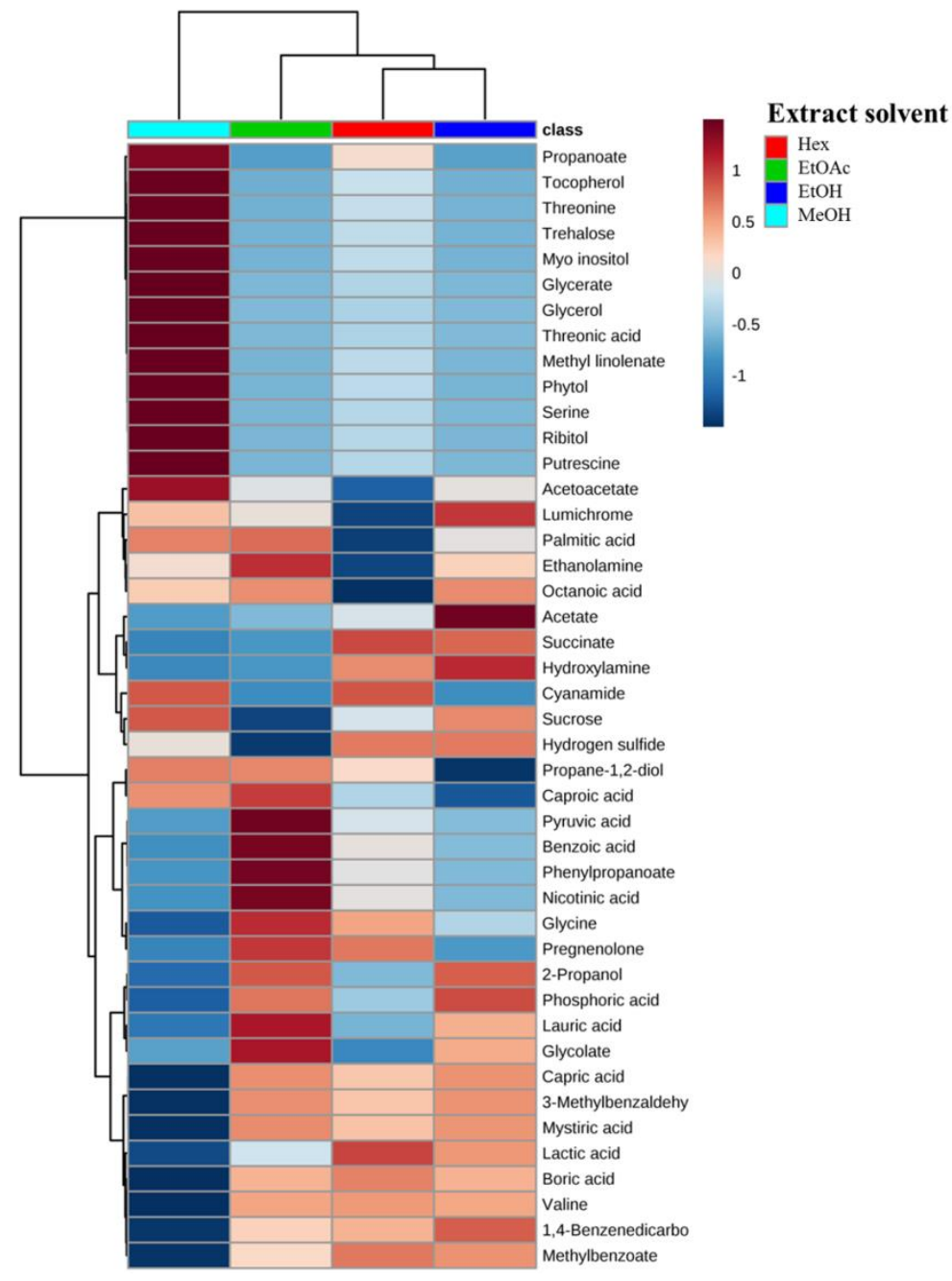

Figure 80: Visualisation of the biologically relevant metabolites identified in each solvent extracts using A) PCA, 2D scores plot showing 95\% confidence regions and B) Hierarchial heatmap visualisation; data is of the mean values of each extraction solvent; $\mathrm{MeOH}, \mathrm{Hex}$, EtOAc and $\mathrm{EtOH}$ from left to right. 
Table 26: Identified metabolites in control C. fritschii extracts (hex, EtOAc, EtOH and MeOH) including chemical class, KEGG pathway and \% normalised abundance across all 4 extracts.

\begin{tabular}{|c|c|c|c|c|c|c|}
\hline Name & Class & Pathway & $\begin{array}{l}\text { Hex } \\
(\%)\end{array}$ & $\begin{array}{l}\text { EtOAc } \\
(\%)\end{array}$ & $\begin{array}{c}\text { EtOH } \\
(\%)\end{array}$ & $\begin{array}{l}\mathrm{MeOH} \\
(\%)\end{array}$ \\
\hline Acetoacetate & Acid & AA degradation & 0 & 23 & 3 & 75 \\
\hline Phenylpropanoate & Acid & Biosynthesis of AA; phe metabolism & 0 & 100 & 0 & 0 \\
\hline Methylbenzoate & Acid & Degradation of aromatics & 2 & 79 & 7 & 11 \\
\hline Boric acid & Acid & $\begin{array}{l}\text { Aminobenzoate degradation, microbial metabolism in diverse } \\
\text { environments }\end{array}$ & 2 & 84 & 6 & 7 \\
\hline Acetate & Acid & Glycolysis, Acetyl-CoA biosynthesis & 0 & 0 & 100 & 0 \\
\hline Pyruvic acid & Acid & Glycolysis, TCA, PPP, AA metabolism & 0 & 100 & 0 & 0 \\
\hline Lactic acid & Acid & Glycolysis/fructose and mannose metabolism, pyruvate metabolism. & 2 & 79 & 6 & 14 \\
\hline Glycolate & Acid & Glyoxylate metabolism, biosynthesis of secondary metabolites & 5 & 86 & 3 & 6 \\
\hline 3-Methylbenzaldehyde & Acid & Microbial metabolism in diverse environments & 7 & 87 & 6 & 0 \\
\hline Phosphoric acid & Acid & PS/oxidative phosphorylation & 3 & 88 & 6 & 3 \\
\hline 2-Propanol & Acid & Propanoate metabolism & 0 & 95 & 5 & 0 \\
\hline Succinate & Acid & $\begin{array}{l}\text { TCA, Pyruvate metabolism, glyoxylate metabolism, biosynthesis of } \\
\text { secondary metabolites }\end{array}$ & 28 & 0 & 72 & 0 \\
\hline Lumichrome & Alcohol & Riboflavin metabolism & 0 & 75 & 21 & 4 \\
\hline Phytol & Alcohol & Chl metabolism & 0 & 0 & 2 & 98 \\
\hline Glycerol & Alcohol & Pentose and glucuronate interconversion & 0 & 0 & 0 & 100 \\
\hline Propane-1,2-diol & Alcohol & Propanoate metabolism & 1 & 86 & 0 & 13 \\
\hline Propanoate & Alcohol & Propanoate metabolism & 0 & 0 & 0 & 100 \\
\hline Threonine & Amino acid & $\begin{array}{l}\text { Biosynthesis of AA; gly, ser, val, leu and ile metabolism, biosynthesis } \\
\text { of secondary metabolites, porphyrin and chl metabolism }\end{array}$ & 0 & 0 & 0 & 100 \\
\hline
\end{tabular}




\begin{tabular}{|c|c|c|c|c|c|c|}
\hline Name & Class & Pathway & $\begin{array}{l}\text { Hex } \\
(\%)\end{array}$ & $\begin{array}{c}\text { EtOAc } \\
(\%)\end{array}$ & $\begin{array}{c}\text { EtOH } \\
(\%)\end{array}$ & $\begin{array}{c}\mathrm{MeOH} \\
(\%)\end{array}$ \\
\hline Valine & Amino acid & $\begin{array}{l}\text { Biosynthesis of AA; val, leu and ile metabolism, biosynthesis of } \\
\text { alkaloids/secondary metabolites }\end{array}$ & 3 & 91 & 6 & 0 \\
\hline Serine & Amino acid & $\begin{array}{l}\text { Biosynthesis of AA; gly, ser and thr metabolism, glyoxylate } \\
\text { metabolism and biosynthesis of secondary metabolites. Chl } \\
\text { biosynthesis, MAA biosynthesis. }\end{array}$ & 0 & 0 & 0 & 100 \\
\hline Glycine & Amino acid & $\begin{array}{l}\text { Biosynthesis of AA; gly, ser and thr metabolism, glyoxylate } \\
\text { metabolism and biosynthesis of secondary metabolites. Chl } \\
\text { biosynthesis, MAA biosynthesis. }\end{array}$ & 4 & 78 & 9 & 9 \\
\hline Nicotinic acid & Aromatic & $\begin{array}{l}\text { Biosynthesis of alkaloids derived from ornithine, lys and nicotinic } \\
\text { acid }\end{array}$ & 0 & 100 & 0 & 0 \\
\hline $\begin{array}{l}1,4- \\
\text { Benzenedicarboxylic } \\
\text { acid }\end{array}$ & Aromatic & Microbial metabolism in diverse environments & 4 & 92 & 4 & 0 \\
\hline Benzoic acid & Aromatic & Phe metabolism and alkaloids derived from shikimate pathway & 2 & 91 & 2 & 5 \\
\hline Ribitol & Aromatic & Riboflavin metabolism & 0 & 0 & 0 & 100 \\
\hline Tocopherol & Aromatic & Ubiquinone and other terpenoid-quinone biosynthesis & 0 & 0 & 0 & 100 \\
\hline Methyl linolenate & Fatty acid & Biosynthesis of unsaturated FA & 0 & 0 & 0 & 100 \\
\hline Caproic acid & Fatty acid & FA biosynthesis & 5 & 78 & 6 & 10 \\
\hline Lauric acid & Fatty acid & FA biosynthesis & 0 & 95 & 5 & 0 \\
\hline Octanoic acid & Fatty acid & $\begin{array}{l}\text { FA biosynthesis, biosynthesis of alkaloids from terpenoid and } \\
\text { polyketides }\end{array}$ & 6 & 77 & 6 & 12 \\
\hline Stearic acid & Fatty acid & FA biosynthesis, biosynthesis of unsaturated FA & 25 & 25 & 25 & 25 \\
\hline Mystiric acid & Fatty acid & FA biosynthesis, biosynthesis of unsaturated FA & 12 & 83 & 5 & 0 \\
\hline Capric acid & Fatty acid & FA biosynthesis, biosynthesis of unsaturated FA & 9 & 85 & 6 & 0 \\
\hline Palmitic acid & Fatty acid & FA biosynthesis, biosynthesis of unsaturated FA & 0 & 81 & 7 & 11 \\
\hline
\end{tabular}




\begin{tabular}{|c|c|c|c|c|c|c|}
\hline Name & Class & Pathway & $\begin{array}{l}\text { Hex } \\
(\%)\end{array}$ & $\begin{array}{c}\text { EtOAc } \\
(\%)\end{array}$ & $\begin{array}{c}\text { EtOH } \\
(\%)\end{array}$ & $\underset{(\%)}{\mathrm{MeOH}}$ \\
\hline Cyanamide & N-compound & Atrazine degradation & 2 & 62 & 8 & 28 \\
\hline Putrescine & $\mathrm{N}$-compound & $\begin{array}{l}\text { Biosynthesis of AA; Arg and pro metabolism, glutathione } \\
\text { metabolism, biosynthesis of alkaloids from ornithine }\end{array}$ & 0 & 0 & 0 & 100 \\
\hline Ethanolamine & N-compound & Glycerophosphlipid metabolism, pyruvate metabolism & 0 & 87 & 4 & 8 \\
\hline Hydroxylamine & N-compound & Nitrogen metabolism & 26 & 0 & 74 & 0 \\
\hline Hydrogen sulfide & S-compound & Sulfur metabolism, microbial metabolism in diverse environments & 3 & 89 & 4 & 4 \\
\hline Pregnenolone & Sterol & Steroid biosynthesis & 5 & 95 & 0 & 0 \\
\hline Myo inositol & Sugar & Galactose metabolism & 0 & 0 & 0 & 100 \\
\hline Sucrose & Sugar & Galactose metabolism, starch and sucrose metabolism & 0 & 0 & 5 & 95 \\
\hline Trehalose & Sugar & Starch and sucrose metabolism & 0 & 0 & 0 & 100 \\
\hline Threonic acid & Sugar acid & Ascorbic acid metabolism & 0 & 0 & 0 & 100 \\
\hline Glycerate & Sugar acid & PPP, gly, ser and thr metabolism, glyoxylate metabolism & 0 & 0 & 0 & 100 \\
\hline
\end{tabular}

*AA $=$ amino acid, $\mathrm{FA}=$ fatty acid, $\mathrm{PPP}=$ pentose phosphate pathway, $\mathrm{TCA}=$ tricarboxylic acid cycle 
From the previous anti-inflammatory experiments, EtOAc, EtOH and $\mathrm{MeOH}$ extracts showed activity by reducing IL-8 secretion in TNF- $\alpha$ stimulated HaCaT cells. In the antibacterial activity assays, $\mathrm{EtOH}$, and $\mathrm{MeOH}$ extracts reduced the growth of $S$. epidermis. 13 metabolites were found in all 4 extracts including; glycine, lactate, glycolate and stearic acid with varying levels. Overal, hex had the lowest normalised abundances across the identified metabolites with the highest abundances found in the EtOAc extract followed by $\mathrm{MeOH}$ and EtOH (Table 26).

8 potentially active metabolites were identified that have uses in beauty personal care (Table 27). The majority of these were found in highest abundances in the EtOAc extract including; palmitic acid, lauric acid, nicotinic acid, glycolic acid and lactic acid. Methyl linoleate, phytol and $\alpha$-tocopherol had the highest abundances in the $\mathrm{MeOH}$ extract.

Table 27: Metabolites identified in $C$. fritschii extracts with potential activity with relevance to beauty personal care.

\begin{tabular}{|c|c|c|}
\hline Metabolite & Potential application/activity & Ref \\
\hline Methyl linoleate & Anti-inflammatory & {$[167]$} \\
\hline Palmitic acid & Anti-inflammatory & {$[168,169]$} \\
\hline Lauric acid & Antibacterial & {$[170]$} \\
\hline Phytol & Anti-inflammatory, antibacterial & {$[171,172]$} \\
\hline$\alpha$-Tocopherol & Antioxidant, anti-inflammatory & {$[173]$} \\
\hline Nicotinic acid & Anti-inflammatory & {$[175]$} \\
\hline Glycolic acid & $\begin{array}{c}\text { Treatment of photo-damaged skin, acne } \\
\text { scars and hyperpigmentation }\end{array}$ \\
\hline Lactic acid & $\begin{array}{c}\text { Treatment of photo-damaged skin, acne } \\
\text { scars and hyperpigmentation }\end{array}$ & {$[175]$} \\
\hline M-gly & Sunscreen, antioxidant & {$[32,175,176]$} \\
\hline
\end{tabular}




\subsection{Discussion}

\subsubsection{Bioactivity assessment of $C$. fritschii for use in beauty personal care}

An anti-inflammatory model using the HaCaT cell line was developed to test activity of extracts from the cyanobacterium, C. fritschii. Initial results show promising activity. Decreased levels in IL-8 secretion were observed with lower concentrations of pooled $C$. fritschii extracts. As many different metabolites are present in the extracts, higher concentrations could mask the anti-inflammatory activity of the sample. No significant increase in IL-8 secretion was observed suggesting that the pooled extracts do not induce inflammation in HaCaT cells.

Individual extracts were then investigated to see which extract was contributing to the reduced IL-8 levels. The lower concentrations were chosen as these showed the most significant decrease in IL-8 secretion (Figure 69). The control EtOAc extract showed activity at all concentrations as well as the $\mathrm{EtOH}$ and $\mathrm{MeOH}$ extracts at 0.5 and $0.1 \mu \mathrm{g}$ $\mathrm{mL}^{-1}$ respectively. No pro-inflammatory response or cell proliferation was observed with the addition of the extracts. Positive antibacterial activity of EtOH and $\mathrm{MeOH}$ extracts from control and UV-B exposed cultures against S. epidermis was also detected.

All four individual control extracts were analysed by GC-MS. Based on the observed results of the in vitro anti-inflammatory (Figure 70) and antibacterial assay (Figure 78). Bioactive metabolites were evaluated for differences comparing EtOAc, EtOH and $\mathrm{MeOH}$ to the hex extract (no activity). Overall, the EtOAc, EtOH and $\mathrm{MeOH}$ extracts had higher normalised abundances than the hex extract. The increased antiinflammatory and antibacterial activity could be due higher levels of potentially active metabolites (Table 27).

Many of the metabolites detected have previously shown bioactivity. Methyl linoleate, present in the $\mathrm{MeOH}$ extract, is a derivative of linoleic acid (omega-6 polyunsaturated fatty acid) and has been seen to have a role in anti-inflammation [167]. The saturated fatty acid, palmitic acid, has been seen to possess anti-inflammatory properties by inhibiting phospholipase $\mathrm{A}_{2}[168,169]$. Lauric acid has demonstrated activity against gram positive bacteria [170]. Phytol, a diterpene member of the acyclic alcohols has been widely studied for bioactivity [171]. This includes anti-inflammatory activity [171] and antimicrobial activity against a variety of bacterial and fungal strains [172]. 
It is also a precursor to $\alpha$-tocopherol (vitamin E). $\alpha$-Tocopherol was found in $\mathrm{MeOH}$ extract and is known to be a free-radical scavenger and also has anti-inflammatory properties [173]. Nicotinic acid (vitamin B3) was found in the EtOAc extract and has been shown to reduce secretion of pro-inflammatory mediators in human monocytes [174]. Other potentially relevant metabolites identified are the $\alpha$-hydroxy acids, lactic acid and glycolic acid. These can be used in formulations to treat photo-damaged skin, to treat acne scars and hyperpigmentation [175].

The drawback with GC-MS is that it is used to identify low molecular weight metabolites. Other high molecular weight metabolites with potential bioactivity, such as polyphenols [178], could be investigated using LC-MS [43]. Combining analytical techniques would increase the pool of identified metabolites.

One of the major bottlenecks of the use of cyanobacteria in industrial biotechnology is the consistent ability to extract high value metabolites at high concentrations for commercial use. Heterologous expression in fast growing E. coli was used to produce m-gly at high enough concentration for bioactivity testing; due to the limited concentration produced by $C$. fritschii. The partially pure m-gly reduced IL-8 secretion within the developed model and although a high error within the experiment was observed, a dose dependent trend was seen. M-gly and other MAAs have been extensively researched for their use as natural sunscreen ingredients due to their strong photoprotective abilities [31], as seen in human skin fibroblast cells [176]. MAAs are known to be multifunctional and also act as antioxidants by quenching radicals [119]. Antioxidant activity of m-gly was shown using the Trolox equivalent antioxidant capacity (TEAC) as well as anti-inflammatory activity in UV exposed HaCaT cells [177].

Results obtained from this chapter give an insight into the potential of $C$. fritschii metabolites as anti-inflammatory and antibacterial agents. 


\subsection{Conclusion}

The development of an anti-inflammatory model was achieved. This model can be used to test a variety of extracts from cyanobacteria and other microorganisms. Combining both bioactivity and metabolic analysis gives an insight into the activity of fractions and the potential metabolites associated with the observed activity. Overall these results show potential for further investigation of anti-inflammatory and antibacterial activity of $C$. fritschii. Further work is needed to verify the results presented in this chapter including the fractionation of active extracts for potential identification of specific active metabolites using a variety of analytical techniques. 


\section{Chapter 6: General discussion}

This chapter discusses the main findings within this thesis (Chapters 2-5) with focus on the relevance to the field, challenges and future work to build on knowledge of the use of cyanobacteria as a sustainable source of industrially relevant ingredients.

Cyanobacterial biotechnology has the potential to solve the demand for naturally sourced environmentally friendly resources as an alternative to fossil fuel derived ingredients for use in industrial biotechnology $[16,21,179]$. The research presented within this thesis adds considerably to current literature on cyanobacterial research at the metabolite level. Investigating at the metabolite level closely relates to the physiology of an organism and is important in determining the adaptation to abiotic stress and production of useful industrially relevant metabolites [43]. The overall aim of this work was to evaluate the metabolite profile of the less investigated cyanobacterium $C$. fritschii as a potential candidate for use in industrial biotechnology with the goal of identifying commonly used and novel metabolites with commercial potential as sustainable ingredients in consumer goods. This was achieved by constructing a GC-MS workflow to evaluate the changes in low molecular weight metabolite levels over varying time-series under standard growth conditions (Chapter 2), between axenic and xenic cultures (Chapter 2) and during UV exposure (UV-A and UV-B, Chapter 3 and 4). Manipulation of abiotic stress such as UV can further increase productivity of industrially relevant metabolites such as MAAs and improve UV tolerance in relevant species $[16,21,22]$ which was also investigated in this thesis (Chapter 4). C. fritschii extracts were also assessed using in-vitro anti-inflammatory, proliferation and antibacterial assays with complementary GC-MS analysis for further elucidation of active ingredients with relevance to personal care products (Chapter 5). The focus was on identifying biologically relevant metabolites with roles in cyanobacterial metabolism for evaluation of growth phases, adaptation to UV stress and applications for use in consumer goods. It is important to investigate these changes in primary metabolites in order to understand secondary metabolite production. Overall, this work builds on the knowledge-base of $C$. fritschii with to my knowledge the first metabolite profiling of $C$. fritschii as measured by GC-MS. 
Cyanobacteria have received much attention for their potential uses in industrial biotechnology [16]. Due to their long evolutionary history they have adaptive capabilities to survive in many extreme environments [6,9]. These adaptations include the production of metabolites that can be exploited in industry as sustainable sources of natural ingredients [16].

Throughout this work, $C$. fritschii has demonstrated the production of metabolites with potential industrial relevance (Chapter 2-5) including active metabolites with relevance to consumer goods (Chapter 5). C. fritschii extracts showed promising activity in in-vitro anti-inflammatory and antibacterial assays in Chapter 5. Combining both bioactivity assessments and GC-MS gave an insight into the potentially active metabolites within each active extract. Metabolites including methyl linoleate, palmitic acid, lauric acid, phytol, $\alpha$-tocopherol, nicotinic acid, glycolic acid and lactic acid have shown previous anti-inflammatory, antibacterial, antioxidant and activity in the treatment of photo-damaged skin which have potential uses in beauty personal care [167-175]. Some of these metabolites also have been identified throughout the experimental analysis of $C$. fritschii. Metabolites such as succinate, fumarate, malate, lactic acid, glycolic acid and pyruvic acid were identified throughout the thesis in various experiments. These can also be exploited in industrial biotechnology as platform chemicals [110]. The photoprotective compounds MAAs were chosen as targeted metabolites with potential applications in industrial biotechnology as natural sunscreens and antioxidants [31]. The MAAs shinorine and m-gly were detected during UV-B exposure in Chapter 3 and during UV-A and UVB exposure in Chapter 4. A major challenge in industrial biotechnology is the ability to produce target valuable compounds in sufficient quantities under repeatable conditions [180]. Different approaches can be considered for increasing cyanobacterial productivity and yield of useful metabolites [132]. This includes the manipulation of abiotic stressors such as UV to increase production of specific metabolites and enhance stress tolerance of specific strains [128]. This was explored in Chapter $\mathbf{4}$ where $C$. fritschii was seen to withstand higher levels of UV-B irradiation after pre-treatment with low dose UV-B suggesting UV stress tolerance and adaptation. Cultures acclimatised to low dose UV (both UV-A and UV-B treatments) had a lower negative impact on metabolism. This indicates the acclimation of cyanobacterial cultures to low dose UV, thus preparing cell metabolism for additional UV exposure. Increased MAA 
levels were observed during UV low dose acclimation followed by shock UV exposure Chapter 4. Other approaches include the genetic manipulation of cyanobacteria to upregulate the production of specific metabolites forming cyanobacterial cell factories [181] and genetic modification of fast-growing microorganisms to include specific biosynthetic gene clusters from cyanobacterial pathways. An example includes the heterologous expression of m-gly gene cluster in E. coil as seen in Chapter 5 [182]. Sufficient yield of the partially pure m-gly extract from $E$. coli was used to test potential anti-inflammatory activity. This showed a promising observational dose response which warrants the further exploration of the bioactive potential of MAAs.

Metabolite profiling can be used to view the physiological changes at the metabolite level in response to changing environments and is a useful tool in cyanobacterial research [43]. The effect of UV-B on cyanobacteria and other photosynthetic organisms has been widely researched with less focus on UV-A exposure. UV-A and UV-B have different effects on photosynthetic organisms and mechanisms of protection vary [27,126]. Within Chapter 3 a significantly higher proportion of metabolites increased in levels during UV-A exposure compared the UV-B study thus showing that supplemented UV-A is less detrimental to $C$. fritschii cells compared to UV-B. Many identified metabolite level changes could be related to stress response as seen in other photosynthetic organisms such as the accumulation of pro which is thought to have a role in providing additional defense as a ROS scavenger and molecular chaperone [122-124]. Accumulation of proline has been observed in Nostoc punctiforme during $24 \mathrm{~h}$ of UV-A stress [52], in the model plant organism Arabidopsis after $24 \mathrm{~h}$ of UV-B treatment [96], and also in C. fritschii after $24 \mathrm{~h}$ of UV-B exposure in Chapter 3. With the depletion of the stratospheric ozone layer much research has been conducted on the effect of increased UV-B levels on photosynthetic organisms $[96,97,183]$. The effect of UV on $C$. fritschii within this work also has relevance within this field by providing an insight into the adaptive response to UV.

Combining intracellular and extracellular analysis is useful in the study of cyanobacteria and other microorganisms providing a more holistic picture of metabolite production during growth and its response to different environmental conditions $[69,70]$. Little work has been undertaken on monitoring both intracellular and extracellular metabolites in cyanobacteria as seen in the UV-B experiment within 
this thesis (Chapter 3). Further experimental analysis would combine both intracellular and extracellular assessment which could be used to monitor and optimise the release of industrially relevant metabolites $[68,69,184]$.

The main bottleneck of this research was the slow growth of axenic $C$. fritschii cultures reducing the amount of biomass available for analysis. Xenic cultures were observed to grow at faster rates and to higher OD. This resulted in the comparison of axenic and xenic cultures. A more realistic approach was taken to use xenic cultures (unialgal) in subsequent experiments. The presence of other microorganisms had a limited effect on the metabolites produced as seen in Chapter $\mathbf{2}$ and would not produce the chosen targeted metabolites MAAs for analysis during UV exposure Chapters 3 and 4. Future research would include the characterisation of the specific microorganisms present within $C$. fritschii cultures, using a combination of metabolomics, metagenomics and transcriptomics [4] followed by controlled co-culture experiments to determine contributions to the metabolites detected [104]. In terms of industrial biotechnology controlled addition of microorganisms, to promote microbial consortia and symbiotic relationships, could results in higher productivity and yield [104].

The limited biomass from cultivation of $C$. fritschii reduced the number of experimental analyses. GC-MS was therefore chosen due to its high sensitivity. By increasing the amount of biomass, more analytical techniques can be utilised to extend the pool of metabolites identified such as LC-MS and NMR.

Time-course metabolomics is becoming a more common approach in evaluating metabolite levels to allow better insight into the physiology of the cells [111]. This approach was used throughout the research to evaluate the changes in metabolite levels over growth and increasing length of UV exposure. As seen in Chapters 2-4 many metabolites were not identified within all time-points due to the high turnover rates of intracellular processes. Growth profiles and metabolite level changes in Chapter 2 (120 d experiment) showed patterns consistent with growth phases as seen in Skeletonema marinoi [94], Chlorella sorokiniana [106] Pharobacter gallaeciensis DSM 17395 [107] and Synechococcus elongates PCC 7942 [108]. With a gap of 8 days between each sampling point much biological data is lost and difficulties are presented in biological interpretation due to a high turnover of intracellular metabolites as previously mentioned. Combination of long-term analysis with shorter term 
sampling points at each growth phase would give a better insight in the changes in metabolite levels.

Missing values in GC-MS analysis is a common drawback [103], here missing values are assumed to be below detection limit and replaced with half of the minimum integrated signal. Evaluation of time-series data provided many challenges. Many aspects of GC-MS data analysis requires manual evaluation but with the availability of AMDIS for spectral deconvolution, NIST and GMD for identification as well as online tools such as SpectConnect [81] for conservative peak alignment and MetaboAnalyst [82] for data visualisation and statistical analysis, data can be analysed with automation from freely available software. The same analysis approach was used throughout for consistency of work in all chapters. Challenges still remain with correct identification of peaks. As an untargeted approach was chosen to evaluate as many metabolites as possible, a match factor of $>60 \%$ was chosen comparing experimental mass spectra to reference library entries. This method successfully identified many chemical compounds with a sub-section relating to cyanobacterial metabolism (level 2 identification [63]), many remained as unknowns (level 4 [63]). During the UV-B experiment (Chapter 4), no biologically relevant metabolites were identified. This could be due to a number of reasons including the identification process, where detected mass spectra were detected with a match factor less than the threshold of $60 \%$ comparing to references. Further investigations of this work would rely on analysis of standards to improve confidence of identifications (level 1 identification [63]). By using a more targeted approach, the analysis of the specific biologically and industrially relevant metabolites identified within this thesis (Chapters 2-5) can be analysed.

Cultivation up to $20 \mathrm{~L}$ under industrial conditions was achieved in Chapter 5. Further work in upscaling to higher volumes is needed to assess metabolite levels and growth parameters for feasibility of industrial scale cultivation. 


\section{Conclusion}

These studies present the first metabolomic data set for $C$. fritschii as measured by GC-MS providing a good base platform for further systems biology. This study is important to build on experimental data already available for cyanobacteria and other photosynthetic organisms and microorganisms during standard growth and UV exposure. The metabolites identified over the time-courses within this thesis represent only a minuscule proportion of the metabolome of $C$. fritschii. By expanding the analytical platforms used and by taking a systems biology approach, through integration of -omics data (metabolomics, transcriptomics and genomics) and in-vitro bioactivity assessment, potential useful pathways and metabolites can be identified. Potential uses of $C$. fritschii in industrial biotechnology can then be further investigated. 


\section{Appendices: Supplementary Tables}

Table S1: Identified intracellular metabolites detected during $28 \mathrm{~d}$ experiment (axenic) including chemical class and possible biosynthetic pathways (KEGG).

\begin{tabular}{|c|c|c|}
\hline Name & Class & Pathway \\
\hline Methylbenzoate & Acid & Degradation of aromatics \\
\hline Glyoxylate & Acid & Glyoxylate metabolism, AA metabolism \\
\hline Boric acid & Acid & Aminobenzoate degradation, microbial metabolism in diverse environments \\
\hline $\begin{array}{l}\text { 3-Hydroxy-L-1-pyrroline-5- } \\
\text { carboxylate }\end{array}$ & Acid & Arg and pro metabolism \\
\hline 3-Hydroxybutanoic acid & Acid & Butanoate metabolism \\
\hline Lactic acid & Acid & Glycolysis/fructose and mannose metabolism, pyruvate metabolism. \\
\hline Glycolate & Acid & Glyoxylate metabolism, biosynthesis of secondary metabolites \\
\hline Oxalate & Acid & Glyoxylate metabolism, biosynthesis of secondary metabolites \\
\hline Phosphoric acid & Acid & PS/oxidative phosphorylation \\
\hline Propanoate & Acid & Propanoate metabolism \\
\hline Fumarate & Acid & TCA, Pyruvate metabolism, glyoxylate metabolism, secondary metabolites \\
\hline Malate & Acid & TCA, Pyruvate metabolism, glyoxylate metabolism, biosynthesis of secondary metabolites \\
\hline Succinate & Acid & TCA, Pyruvate metabolism, glyoxylate metabolism, biosynthesis of secondary metabolites \\
\hline 1-Butanol & Alcohol & Butanoate metabolism, microbial metabolism, degradation of aromatic compounds \\
\hline Phytol & Alcohol & Chl metabolism \\
\hline Propane-1,3-diol & Alcohol & Glycerolipid metabolism \\
\hline Ethane-1,2-diol & Alcohol & Glyoxylate and dicarboxylate metabolism \\
\hline Glycerol & Alcohol & Pentose and glucuronate interconversion \\
\hline 2-Propanol & Alcohol & Propanoate metabolism \\
\hline Propane-1,2-diol & Alcohol & Propanoate metabolism \\
\hline
\end{tabular}




\begin{tabular}{|c|c|c|}
\hline Name & Class & Pathway \\
\hline Ornithine & Amino acid & $\begin{array}{l}\text { Biosynthesis of AA and seconary metabolites. Arg and pro metabolism. Glutathione } \\
\text { metabolism }\end{array}$ \\
\hline Proline & Amino acid & Biosynthesis of AA; Arg and pro biosynthesis, secondary metabolite biosynthesis \\
\hline Tyrosine & Amino acid & $\begin{array}{l}\text { Biosynthesis of AA and alkaloids derived from shikimate pathway, terpernoid-quinone } \\
\text { biosynthesis }\end{array}$ \\
\hline Glutamate & Amino acid & $\begin{array}{l}\text { Biosynthesis of AA; arg, ala, asp and glu, arg and pro metabolism. Forms L-ornithine/L- } \\
\text { glutamine }\end{array}$ \\
\hline Lysine & Amino acid & Biosynthesis of AA and biosynthesis of alkaloids \\
\hline Threonine & Amino acid & $\begin{array}{l}\text { Biosynthesis of AA; gly, ser, val, leu and ile metabolism, biosynthesis of secondary } \\
\text { metabolites, porphyrin and chl metabolism }\end{array}$ \\
\hline Isoleucine & Amino acid & $\begin{array}{l}\text { Biosynthesis of AA; gly, ser, val, leu and ile metabolism, biosynthesis of } \\
\text { alkaloids/secondary metabolites }\end{array}$ \\
\hline Valine & Amino acid & $\begin{array}{l}\text { Biosynthesis of AA; val, leu and ile metabolism, biosynthesis of alkaloids/secondary } \\
\text { metabolites }\end{array}$ \\
\hline Aspartate & Amino acid & $\begin{array}{l}\text { Biosynthesis of AA; ala, asp and glu metabolism. Arg, gly, ser and thr metabolism, cys } \\
\text { and met metabolism. Carbon fixation }\end{array}$ \\
\hline Arginine & Amino acid & Biosynthesis of AA; Arg and pro biosynthesis, secondary metabolite biosynthesis \\
\hline Serine & Amino acid & $\begin{array}{l}\text { Biosynthesis of AA; gly, ser and thr metabolism, glyoxylate metabolism and biosynthesis } \\
\text { of secondary metabolites. Chl biosynthesis, MAA biosynthesis. }\end{array}$ \\
\hline Glycine & Amino acid & $\begin{array}{l}\text { Biosynthesis of AA; gly, ser and thr metabolism, glyoxylate metabolism and biosynthesis } \\
\text { of secondary metabolites. Chl biosynthesis, MAA biosynthesis. }\end{array}$ \\
\hline Phenylalanine & Amino acid & $\begin{array}{l}\text { Biosynthesis of AA. Phe metabolism via shikimate pathway, Tyr metabolism, biosynthesis } \\
\text { of alkaloids derived from shikimate pathway and ornithine. }\end{array}$ \\
\hline 5-Oxoproline & Amino acid & Glutathione metabolism \\
\hline Cyanuric acid & Aromatic & Atrazine degredation \\
\hline Histamine & Aromatic & Biosynthesis of alkaloids derived from histidine and pyrine, secondary metabolites \\
\hline Benzoic acid & Aromatic & Phe metabolism and alkaloids derived from shikimate pathway \\
\hline Lumichrome & Aromatic & Riboflavin metabolism \\
\hline
\end{tabular}




\begin{tabular}{|c|c|c|}
\hline Name & Class & Pathway \\
\hline Tocopherol & Aromatic & Ubiquinone and other terpenoid-quinone biosynthesis \\
\hline 4-Methylbenzaldehyde & Aromatic & Xylene degredation \\
\hline Octanoic acid & Ester & FA biosynthesis, biosynthesis of alkaloids from terpenoid and polyketides \\
\hline Lauric acid & Fatty acid & FA biosynthesis \\
\hline Mystiric acid & Fatty acid & FA biosynthesis, biosynthesis of unsaturated FA \\
\hline Palmitic acid & Fatty acid & FA biosynthesis, biosynthesis of unsaturated FA \\
\hline Stearic acid & Fatty acid & FA biosynthesis, biosynthesis of unsaturated FA \\
\hline Eicosanoic acid & Fatty acid & FA biosynthesis, biosynthesis of unsaturated FA \\
\hline Ethanolamine & N-compound & Glycerophosphlipid metabolism, pyruvate metabolism \\
\hline Hydroxylamine & N-compound & Nitrogen metabolism \\
\hline Sperimine & N-compound & Arg and pro metabolism, glutathione metabolism \\
\hline Sperimidine & N-compound & Arg and pro metabolism, glutathione metabolism, phenlpropanoid metabolism \\
\hline Adenosine & Nucleoside & Purine metabolism \\
\hline Cyanamide & Other & Atrazine degradation \\
\hline Hydrogen sulfide & S-compound & Sulfur metabolism, microbial metabolism in diverse environments \\
\hline Rhamnose & Sugar & Fructose and mannose metabolism \\
\hline Mannose & Sugar & $\begin{array}{l}\text { Fructose and mannose metabolism, amino sugar and nucleotide metabolism, galactose } \\
\text { metabolism }\end{array}$ \\
\hline Fructose & Sugar & Fructose and mannose metabolism, galactose metabolism, starch and sucrose metabolism \\
\hline Galactose & Sugar & Galactose metabolism \\
\hline Raffinose & Sugar & Galactose metabolism \\
\hline Sucrose & Sugar & Galactose metabolism, starch and sucrose metabolism \\
\hline Lyxose & Sugar & Pentose and glucuronate interconversion \\
\hline Trehalose & Sugar & Starch and sucrose metabolism \\
\hline Xylulose-5-phosphate & Sugar phosphate & Pentose phosphate pathway \\
\hline
\end{tabular}

*AA = amino acid, FA = fatty acid, $\mathrm{PPP}=$ pentose phosphate pathway, $\mathrm{TCA}=$ tricarboxylic acid cycle 
Table S2: Identified intracellular metabolites detected during $28 \mathrm{~d}$ experiment (xenic) including chemical class and possible biosynthetic pathways (KEGG).

\begin{tabular}{|c|c|c|}
\hline Name & Class & Pathway \\
\hline Methylbenzoate & Acid & Degradation of aromatics \\
\hline Boric acid & Acid & Aminobenzoate degradation, microbial metabolism in diverse environments \\
\hline $\begin{array}{l}\text { 3-Hydroxy-L-1-pyrroline-5- } \\
\text { carboxylate }\end{array}$ & Acid & Arg and pro metabolism \\
\hline 2-Oxobutanoate & Acid & $\begin{array}{l}\text { Biosynthesis of AA; cys and met metabolism, thr metabolism. Val, leu and ile } \\
\text { biosynthesis }\end{array}$ \\
\hline 3-Hydroxybutanoic acid & Acid & Butanoate metabolism \\
\hline Malonate & Acid & FA biosynthesis \\
\hline 5-Oxoproline & Acid & Glutathione metabolism \\
\hline Lactic acid & Acid & Glycolysis/fructose and mannose metabolism, pyruvate metabolism. \\
\hline Oxalate & Acid & Glyoxylate metabolism, biosynthesis of secondary metabolites \\
\hline Phosphoric acid & Acid & PS/oxidative phosphorylation \\
\hline Propanoate & Acid & Propanoate metabolism \\
\hline Fumarate & Acid & TCA, Pyruvate metabolism, glyoxylate metabolism, secondary metabiltes \\
\hline Malate & Acid & TCA, Pyruvate metabolism, glyoxylate metabolism, biosynthesis of secondary metabolites \\
\hline Succinate & Acid & TCA, Pyruvate metabolism, glyoxylate metabolism, biosynthesis of secondary metabolites \\
\hline 1-Butanol & Alcohol & Butanoate metabolism, microbial metabolism, degradation of aromatic compounds \\
\hline Phytol & Alcohol & Chl metabolism \\
\hline Propane-1,3-diol & Alcohol & Glycerolipid metabolism \\
\hline Ethane-1,2-diol & Alcohol & Glyoxylate and dicarboxylate metabolism \\
\hline Glycerol & Alcohol & Pentose and glucuronate interconversion \\
\hline 2-Propanol & Alcohol & Propanoate metabolism \\
\hline Propane-1,2-diol & Alcohol & Propanoate metabolism \\
\hline
\end{tabular}




\begin{tabular}{|c|c|c|}
\hline Name & Class & Pathway \\
\hline Ornithine & Amino acid & $\begin{array}{l}\text { Biosynthesis of AA and seconary metabolites. Arg and pro metabolism. Glutathione } \\
\text { metabolism }\end{array}$ \\
\hline Alanine & Amino acid & $\begin{array}{l}\text { Biosynthesis of AA; ala, asp and glu metabolism, cys and met metabolism, carbon fixation } \\
\text { and biosynthesis of secondary metabolites }\end{array}$ \\
\hline Glutamate & Amino acid & $\begin{array}{l}\text { Biosynthesis of AA; arg, ala, asp and glu, arg and pro metabolism. Forms L-ornithine/L- } \\
\text { glutamine }\end{array}$ \\
\hline Lysine & Amino acid & Biosynthesis of AA and biosynthesis of alkaloids \\
\hline Threonine & Amino acid & $\begin{array}{l}\text { Biosynthesis of AA; gly, ser, val, leu and ile metabolism, biosynthesis of secondary } \\
\text { metabolites, porphyrin and chl metabolism }\end{array}$ \\
\hline Valine & Amino acid & $\begin{array}{l}\text { Biosynthesis of AA; val, leu and ile metabolism, biosynthesis of alkaloids/secondary } \\
\text { metabolites }\end{array}$ \\
\hline Aspartate & Amino acid & $\begin{array}{l}\text { Biosynthesis of AA; ala, asp and glu metabolism. Arg, gly, ser and thr metabolism, cys } \\
\text { and met metabolism. Carbon fixation }\end{array}$ \\
\hline Serine & Amino acid & $\begin{array}{l}\text { Biosynthesis of AA; gly, ser and thr metabolism, glyoxylate metabolism and biosynthesis } \\
\text { of secondary metabolites. Chl biosynthesis, MAA biosynthesis. }\end{array}$ \\
\hline Glycine & Amino acid & $\begin{array}{l}\text { Biosynthesis of AA; gly, ser and thr metabolism, glyoxylate metabolism and biosynthesis } \\
\text { of secondary metabolites. Chl biosynthesis, MAA biosynthesis. }\end{array}$ \\
\hline Phenylalanine & Amino acid & $\begin{array}{l}\text { Biosynthesis of AA. Phe metabolism via shikimate pathway, Tyr metabolism, biosynthesis } \\
\text { of alkaloids derived from shikimate pathway and ornithine. }\end{array}$ \\
\hline Arginine & Amino acid & Biosynthesis of AA; Arg and pro biosynthesis, secondary metabolite biosynthesis \\
\hline Proline & Amino acid & Biosynthesis of AA; Arg and pro biosynthesis, secondary metabolite biosynthesis \\
\hline Histamine & Aromatic & Biosynthesis of alkaloids derived from histidine and pyrine, secondary metabolites \\
\hline Benzoic acid & Aromatic & Phe metabolism and alkaloids derived from shikimate pathway \\
\hline Lumichrome & Aromatic & Riboflavin metabolism \\
\hline 3-Hydroxyphenylacetate & Aromatic & Tyr and phe metabolism \\
\hline Tocopherol & Aromatic & Ubiquinone and other terpenoid-quinone biosynthesis \\
\hline Octanoic acid & Ester & FA biosynthesis, biosynthesis of alkaloids from terpenoid and polyketides \\
\hline Eicosanoic acid & Fatty acid & FA biosynthesis, biosynthesis of unsaturated FA \\
\hline
\end{tabular}




\begin{tabular}{lll}
\hline Name & Class & Pathway \\
\hline Lauric acid & Fatty acid & FA biosynthesis \\
Capric acid & Fatty acid & FA biosynthesis, biosynthesis of unsaturated FA \\
Mystiric acid & Fatty acid & FA biosynthesis, biosynthesis of unsaturated FA \\
Palmitic acid & Fatty acid & FA biosynthesis, biosynthesis of unsaturated FA \\
Stearic acid & Fatty acid & FA biosynthesis, biosynthesis of unsaturated FA \\
Putrescine & N-compound & Biosynthesis of AA; Arg and pro metabolism, glutathione metabolism, biosynthesis of \\
Ethanolamine & N-compound & alkaloids from ornithine \\
Urea & N-compound & Metabolic pathways, microbial metabolism \\
Hydroxylamine & N-compound & Nitrogen metabolism \\
Sperimidine & N-compound & Arg and pro metabolism, glutathione metabolism, phenlpropanoid metabolism \\
Adenosine & Nucleoside & Purine metabolism \\
Hydrogen sulfide & S-compound & Sulfur metabolism, microbial metabolism in diverse environments \\
Rhamnose & Sugar & Fructose and mannose metabolism \\
Glucose & Sugar & Glycolysis, PPP, biosynthesis of secondary metabolites. \\
Raffinose & Sugar & Galactose metabolism \\
Sucrose & Sugar & Galactose metabolism, starch and sucrose metabolism \\
Lyxose & Sugar & Pentose and glucuronate interconversion \\
Trehalose & Sugar & Starch and sucrose metabolism \\
Xylulose-5-phosphate & Sugar phosphate & Pentose phosphate pathway \\
\hline$*$ AA = amino acid, FA = fatty acid, PPP = pentose phosphate pathway, TCA=tricarboxylic acid cycle &
\end{tabular}


Table S3: Identified intracellular metabolites detected during $120 \mathrm{~d}$ experiment including chemical class and possible biosynthetic pathways (KEGG).

\begin{tabular}{|c|c|c|}
\hline Name & Class & Pathway \\
\hline Methylbenzoate & Acid & Degradation of aromatics \\
\hline Lactic acid & Acid & Glycolysis/fructose and mannose metabolism, pyruvate metabolism. \\
\hline $\begin{array}{l}\text { 3-Hydroxy-L-1-pyrroline-5- } \\
\text { carboxylate }\end{array}$ & Acid & Arg and pro metabolism \\
\hline Adipic acid 2TMS & Acid & Biosynthesis of AA \\
\hline 4-Hydroxybutyric acid & Acid & Carbon fixation pathways in prokaryotes \\
\hline Oxalate & Acid & Glyoxylate metabolism, biosynthesis of secondary metabolites \\
\hline Glycolate & Acid & Glyoxylate metabolism, biosynthesis of secondary metabolites \\
\hline 4-Methoxycinnamic acid & Acid & Phenylpropanoid biosynthesis \\
\hline Phosphoric acid & Acid & PS/oxidative phosphorylation \\
\hline Propanoate & Acid & Propanoate metabolism \\
\hline Fumarate & Acid & TCA, Pyruvate metabolism, glyoxylate metabolism, secondary metabiltes \\
\hline Malate & Acid & TCA, Pyruvate metabolism, glyoxylate metabolism, biosynthesis of secondary metabolites \\
\hline Citrate & Acid & TCA, Pyruvate metabolism, glyoxylate metabolism, biosynthesis of secondary metabolites \\
\hline Succinate & Acid & TCA, Pyruvate metabolism, glyoxylate metabolism, biosynthesis of secondary metabolites \\
\hline 1-Butanol & Alcohol & Butanoate metabolism, microbial metabolism, degradation of aromatic compounds \\
\hline Phytol & Alcohol & Chl metabolism \\
\hline Glycerol & Alcohol & Pentose and glucuronate interconversion \\
\hline Ornithine & Amino acid & $\begin{array}{l}\text { Biosynthesis of AA and seconary metabolites. Arg and pro metabolism. Glutathione } \\
\text { metabolism }\end{array}$ \\
\hline Glutamate & Amino acid & $\begin{array}{l}\text { Biosynthesis of AA; arg, ala, asp and glu, arg and pro metabolism. Forms L-ornithine/L- } \\
\text { glutamine }\end{array}$ \\
\hline Lysine & Amino acid & Biosynthesis of AA and biosynthesis of alkaloids \\
\hline Valine & Amino acid & Biosynthesis of AA; val, leu and ile metabolism, biosynthesis of alkaloids \\
\hline
\end{tabular}




\begin{tabular}{|c|c|c|}
\hline Name & Class & Pathway \\
\hline Aspartate & Amino acid & $\begin{array}{l}\text { Biosynthesis of AA; ala, asp and glu metabolism. Arg, gly, ser and thr metabolism, cys } \\
\text { and met metabolism. Carbon fixation }\end{array}$ \\
\hline Glycine & Amino acid & $\begin{array}{l}\text { Biosynthesis of AA; gly, ser and thr metabolism, glyoxylate metabolism and biosynthesis } \\
\text { of secondary metabolites. Chl biosynthesis, MAA biosynthesis. }\end{array}$ \\
\hline Phenylalanine & Amino acid & $\begin{array}{l}\text { Biosynthesis of AA. Phe metabolism via shikimate pathway, Tyr metabolism, biosynthesis } \\
\text { of alkaloids derived from shikimate pathway and ornithine. }\end{array}$ \\
\hline Proline & Amino acid & Biosynthesis of AA; Arg and pro biosynthesis, secondary metabolite biosynthesis \\
\hline Cyanuric acid & Aromatic & Atrazine degredation \\
\hline Hydroxyquinol & Aromatic & Benzoate degradation \\
\hline Benzoic acid & Aromatic & Phe metabolism and alkaloids derived from shikimate pathway \\
\hline $\begin{array}{l}\text { 1-Hydroxy-3-methoxy-6- } \\
\text { methylanthraquinone }\end{array}$ & Aromatic & Polyketide synthesis \\
\hline $\begin{array}{l}\text { 4-Hydroxyanthraquinone-2- } \\
\text { carboxylic acid }\end{array}$ & Aromatic & Polyketide synthesis \\
\hline Adenine & Aromatic & Purine metabolism \\
\hline lumichrome & Aromatic & Riboflavin metabolism \\
\hline Lauric acid & Fatty acid & FA biosynthesis \\
\hline Caprylic acid & Fatty acid & FA biosynthesis, biosynthesis of alkaloids from terpenoid and polyketide \\
\hline Eicosanoic acid & Fatty acid & FA biosynthesis, biosynthesis of unsaturated FA \\
\hline Capric acid & Fatty acid & FA biosynthesis, biosynthesis of unsaturated FA \\
\hline Mystiric acid & Fatty acid & FA biosynthesis, biosynthesis of unsaturated FA \\
\hline Palmitoleic acid & Fatty acid & FA biosynthesis, biosynthesis of unsaturated FA \\
\hline Palmitic acid & Fatty acid & FA biosynthesis, biosynthesis of unsaturated FA \\
\hline Oleic acid & Fatty acid & FA biosynthesis, biosynthesis of unsaturated FA \\
\hline Stearic acid & Fatty acid & FA biosynthesis, biosynthesis of unsaturated FA \\
\hline Ethanolamine & N-compound & Glycerophosphlipid metabolism, pyruvate metabolism \\
\hline Urea & N-compound & Metabolic pathways, microbial metabolism \\
\hline
\end{tabular}




\begin{tabular}{lll}
\hline Name & Class & Pathway \\
\hline Hydroxylamine & N-compound & Nitrogen metabolism \\
Adenosine & Nucleoside & Purine metabololism \\
Hydrogen sulfide & S-compound & Sulfur metabolism, microbial metabolism in diverse environments \\
Sorbose & Sugar & Fructose and mannose metabolism \\
Fructose & Sugar & Fructose and mannose metabolism, galactose metabolism, starch and sucrose metabolism \\
Galactose & Sugar & Galactose metabolism \\
Raffinose & Sugar & Galactose metabolism \\
Sucrose & Sugar & Galactose metabolism, starch and sucrose metabolism \\
Glucose & Sugar & Glycolysis, PPP biosynthesis of secondary metabolites \\
Trehalose & Sugar & Starch and sucrose metabolism \\
Glycerate & Sugar acid & PPP, gly, ser and thr metaboim, glyoxylate metabolism \\
Glycerol 3-phosphate & Sugar phosphate & Glycerolipid/glycerophospholipid metabolism, metabolism of secondary metabolites \\
\hline *AA =amino acid, FA = fatty acid, PPP = pentose phosphate pathway, TCA=tricarboxylic acid cycle \\
\hline
\end{tabular}


Table S4: Identified intracellular metabolites detected during $48 \mathrm{~h}$ of PAR only including chemical class and possible biosynthetic pathways (KEGG).

\begin{tabular}{|c|c|c|}
\hline Name & Class & Pathway \\
\hline Boric acid & Acid & Aminobenzoate degradation-microbial metabolism in diverse environments \\
\hline $\begin{array}{l}\text { 3-Hydroxy-L-1-pyrroline-5- } \\
\text { carboxylate }\end{array}$ & Acid & Arg and pro metabolism \\
\hline 5-Oxoproline & Acid & Glutathione metabolism \\
\hline Phosphoric acid & Acid & PS/oxidative phosphorylation \\
\hline Gluconic acid-1,4-lactone & Acid & PPP \\
\hline Glycerate & Acid & PPP, gly, ser and thr metaboim, glyoxylate metabolism \\
\hline Malate & Acid & TCA, Pyruvate metabolism, glyoxylate metabolism, biosynthesis of secondary metabolites \\
\hline Citrate & Acid & TCA, Pyruvate metabolism, glyoxylate metabolism, biosynthesis of secondary metabolites \\
\hline Glycerol & Alcohol & Pentose and glucuronate interconversion \\
\hline Propan-2-ol & Alcohol & Propanoate metabolism \\
\hline Ornithine & Amino acid & $\begin{array}{l}\text { Biosynthesis of AA and seconary metabolites. Arg and pro metabolism. Glutathione } \\
\text { metabolism }\end{array}$ \\
\hline Alanine & Amino acid & $\begin{array}{l}\text { Biosynthesis of AA; ala, asp and glu metabolism, cys and met metabolism, carbon fixation } \\
\text { and biosynthesis of secondary metabolites }\end{array}$ \\
\hline Tyrosine & Amino acid & $\begin{array}{l}\text { Biosynthesis of AA and alkaloids derived from shikimate pathway, terpernoid-quinone } \\
\text { biosynthesis }\end{array}$ \\
\hline Glutamate & Amino acid & $\begin{array}{l}\text { Biosynthesis of AA; arg, ala, asp and glu, arg and pro metabolism. Forms L-ornithine/L- } \\
\text { glutamine }\end{array}$ \\
\hline Lysine & Amino acid & Biosynthesis of AA and biosynthesis of alkaloids \\
\hline Methionine & Amino acid & Biosynthesis of AA; cys and met metabolism and secondary metabolism \\
\hline Threonine & Amino acid & $\begin{array}{l}\text { Biosynthesis of AA; gly, ser, val, leu and ile metabolism, biosynthesis of secondary } \\
\text { metabolites, porphyrin and chl metabolism }\end{array}$ \\
\hline Leucine & Amino acid & $\begin{array}{l}\text { Biosynthesis of AA; gly, ser, val, leu and ile metabolism, biosynthesis of } \\
\text { alkaloids/secondary metabolites }\end{array}$ \\
\hline
\end{tabular}




\begin{tabular}{|c|c|c|}
\hline Name & Class & Pathway \\
\hline Isoleucine & Amino acid & $\begin{array}{l}\text { Biosynthesis of AA; gly, ser, val, leu and ile metabolism, biosynthesis of } \\
\text { alkaloids/secondary metabolites }\end{array}$ \\
\hline Aspartate & Amino acid & $\begin{array}{l}\text { Biosynthesis of AA; ala, asp and glu metabolism. Arg, gly, ser and thr metabolism, cys } \\
\text { and met metabolism. Carbon fixation }\end{array}$ \\
\hline Serine & Amino acid & $\begin{array}{l}\text { Biosynthesis of AA; gly, ser and thr metabolism, glyoxylate metabolism and biosynthesis } \\
\text { of secondary metabolites. Chl biosynthesis, MAA biosynthesis. }\end{array}$ \\
\hline Glycine & Amino acid & $\begin{array}{l}\text { Biosynthesis of AA; gly, ser and thr metabolism, glyoxylate metabolism and biosynthesis } \\
\text { of secondary metabolites. Chl biosynthesis, MAA biosynthesis. }\end{array}$ \\
\hline Phenylalanine & Amino acid & $\begin{array}{l}\text { Biosynthesis of AA. Phe metabolism via shikimate pathway, Tyr metabolism, biosynthesis } \\
\text { of alkaloids derived from shikimate pathway and ornithine. }\end{array}$ \\
\hline Cyanuric acid & Aromatic & Atrazine degredation \\
\hline Palmitic acid & Fatty acid & FA biosynthesis, biosynthesis of unsaturated FA \\
\hline Stearic acid & Fatty acid & FA biosynthesis, biosynthesis of unsaturated FA \\
\hline Hydroxylamine & N-compound & Nitrogen metabolism \\
\hline Adenosine & Nucleoside & Purine metabolism \\
\hline Cyanamide & Other & Atrazine degradation \\
\hline Hydrogen sulfide & S-compound & Sulfur metabolism, microbial metabolism in diverse environments \\
\hline Sorbose & Sugar & Fructose and mannose metabolism \\
\hline Rhamnose & Sugar & Fructose and mannose metabolism \\
\hline Fructose & Sugar & Fructose and mannose metabolism, galactose metabolism, starch and sucrose metabolism \\
\hline Sucrose & Sugar & Galactose metabolism, starch and sucrose metabolism \\
\hline Glucose & Sugar & Glycolysis, PPP biosynthesis of secondary metabolites \\
\hline Trehalose & Sugar & Starch and sucrose metabolism \\
\hline Mannose 6-phosphate & Sugar phosphate & Fructose and mannose metabolism, synthesis of secondary metabolites \\
\hline Glycerol 3-phosphate & Sugar phosphate & Glycerolipid/glycerophospholipid metabolism, metabolism of secondary metabolites \\
\hline
\end{tabular}


Table S5: Identified intracellular and extracellular metabolites detected during UV-B + PAR including chemical class and possible biosynthetic pathways (KEGG).

\begin{tabular}{|c|c|c|c|}
\hline Name & Class & Pathway & $\begin{array}{l}\text { Intra/Extra- } \\
\text { cellular }\end{array}$ \\
\hline Boric acid & Acid & Aminobenzoate degradation, microbial metabolism in diverse environments & Intra \\
\hline Phosphoric acid & Acid & PS/oxidative phosphorylation & Intra \\
\hline 2-Oxobutanoate & Acid & $\begin{array}{l}\text { Biosynthesis of AA; cys and met metabolism, thr metabolism. Val, leu and } \\
\text { ile biosynthesis }\end{array}$ & Intra \\
\hline 4-Hydroxybutyric acid & Acid & Carbon fixation pathways in prokaryotes & Intra \\
\hline Methylbenzoate & Acid & Degradation of aromatics & Intra \\
\hline 5-Oxoproline & Acid & Glutathione metabolism & Intra \\
\hline Pyruvic acid & Acid & Glycolysis, TCA, PPP, AA metabolism & Intra \\
\hline Lactic acid & Acid & Glycolysis/fructose and mannose metabolism, pyruvate metabolism. & Intra \\
\hline Oxalate & Acid & Glyoxylate metabolism, biosynthesis of secondary metabolites & Intra \\
\hline Methylmalonate & Acid & Pyrimidine metabolism, AA degredation, propanoate metabolism. & Intra \\
\hline Butane-2,3-diol & Alcohol & Butanoate metabolism & Intra \\
\hline Ethane-1,2-diol & Alcohol & Glyoxylate and dicarboxylate metabolism & Intra \\
\hline 1-Propanol & Alcohol & Propanoate metabolism & Intra \\
\hline 2-Propanol & Alcohol & Propanoate metabolism & Intra \\
\hline Tyrosine & Amino acid & $\begin{array}{l}\text { Biosynthesis of AA and alkaloids derived from shikimate pathway, } \\
\text { terpernoid-quinone biosynthesis }\end{array}$ & Intra \\
\hline Serine & Amino acid & $\begin{array}{l}\text { Biosynthesis of AA; gly, ser and thr metabolism, glyoxylate metabolism } \\
\text { and biosynthesis of secondary metabolites. Chl biosynthesis, MAA } \\
\text { biosynthesis. }\end{array}$ & Intra \\
\hline Glycine & Amino acid & $\begin{array}{l}\text { Biosynthesis of AA; gly, ser and thr metabolism, glyoxylate metabolism } \\
\text { and biosynthesis of secondary metabolites. Chl biosynthesis, MAA } \\
\text { biosynthesis. }\end{array}$ & Intra \\
\hline
\end{tabular}




\begin{tabular}{|c|c|c|c|}
\hline Name & Class & Pathway & $\begin{array}{l}\text { Intra/Extra- } \\
\text { cellular }\end{array}$ \\
\hline Phenylalanine & Amino acid & $\begin{array}{l}\text { Biosynthesis of AA. Phe metabolism via shikimate pathway, Tyr } \\
\text { metabolism, biosynthesis of alkaloids derived from shikimate pathway and } \\
\text { ornithine. }\end{array}$ & Intra \\
\hline Proline & Amino acid & $\begin{array}{l}\text { Biosynthesis of AA; Arg and pro biosynthesis, secondary metabolite } \\
\text { biosynthesis }\end{array}$ & Intra \\
\hline Glutamate & Amino acid & $\begin{array}{l}\text { Biosynthesis of AA; arg, ala, asp and glu, arg and pro metabolism. Forms } \\
\text { L-ornithine/L-glutamine }\end{array}$ & Intra \\
\hline 4-Methylbenzaldehyde & Aromatic & Xylene degredation & Intra \\
\hline Mystiric acid & Fatty acid & FA biosynthesis, biosynthesis of unsaturated FA & Intra \\
\hline Palmitic acid & Fatty acid & FA biosynthesis, biosynthesis of unsaturated FA & Intra \\
\hline Stearic acid & Fatty acid & FA biosynthesis, biosynthesis of unsaturated FA & Intra \\
\hline Ethanolamine & N-compound & Glycerophosphlipid metabolism, pyruvate metabolism & Intra \\
\hline Hydroxylamine & N-compound & Nitrogen metabolism & Intra \\
\hline Mannose & Sugar & $\begin{array}{l}\text { Fructose and mannose metabolism, amino sugar and nucleotide metabolism, } \\
\text { galactose metabolism }\end{array}$ & Intra \\
\hline D-Glucose & Sugar & Glycolysis, PPP biosynthesis of secondary metabolites. & Intra \\
\hline 2-Oxobutanoate & Acid & $\begin{array}{l}\text { Biosynthesis of AA; cys and met metabolism, thr metabolism. Val, leu and } \\
\text { ile biosynthesis }\end{array}$ & Extra \\
\hline Glycolate & Acid & Glyoxylate metabolism, biosynthesis of secondary metabolites & Extra \\
\hline Citrate & Acid & $\begin{array}{l}\text { TCA, Pyruvate metabolism, glyoxylate metabolism, biosynthesis of } \\
\text { secondary metabolites }\end{array}$ & Extra \\
\hline Fumarate & Acid & TCA, Pyruvate metabolism, glyoxylate metabolism, secondary metabiltes & Extra \\
\hline Malate & Acid & $\begin{array}{l}\text { TCA, Pyruvate metabolism, glyoxylate metabolism, biosynthesis of } \\
\text { secondary metabolites }\end{array}$ & Extra \\
\hline Succinate & Acid & $\begin{array}{l}\text { TCA, Pyruvate metabolism, glyoxylate metabolism, biosynthesis of } \\
\text { secondary metabolites }\end{array}$ & Extra \\
\hline Oxalate & Acid & Glyoxylate metabolism, biosynthesis of secondary metabolites & Extra \\
\hline
\end{tabular}




\begin{tabular}{|c|c|c|c|}
\hline Name & Class & Pathway & $\begin{array}{l}\text { Intra/Extra- } \\
\text { cellular }\end{array}$ \\
\hline Benzoic acid & Acid & Phe metabolism and alkaloids derived from shikimate pathway & Extra \\
\hline Gluconic lactone & Acid & PPP & Extra \\
\hline Arabitol & Alcohol & Pentose and glucuronate interconversion & Extra \\
\hline Glycerol & Alcohol & Pentose and glucuronate interconversion & Extra \\
\hline Leucine & Amino acid & $\begin{array}{l}\text { Biosynthesis of AA; gly, ser, val, leu and ile metabolism, biosynthesis of } \\
\text { alkaloids/secondary metabolites }\end{array}$ & Extra \\
\hline Ethanolamine & Amino alcohol & Glycerophosphlipid metabolism, pyruvate metabolism & Extra \\
\hline Tocopherol & Aromatic & Ubiquinone and other terpenoid-quinone biosynthesis & Extra \\
\hline Lauric acid & Fatty acid & FA biosynthesis & Extra \\
\hline Octanoic acid & Fatty acid & FA biosynthesis, biosynthesis of alkaloids from terpenoid and polyketides & Extra \\
\hline Capric acid & Fatty acid & FA biosynthesis, biosynthesis of unsaturated FA & Extra \\
\hline Mystiric acid & Fatty acid & FA biosynthesis, biosynthesis of unsaturated FA & Extra \\
\hline Palmitic acid & Fatty acid & FA biosynthesis, biosynthesis of unsaturated FA & Extra \\
\hline Stearic acid & Fatty acid & FA biosynthesis, biosynthesis of unsaturated FA & Extra \\
\hline Putrescine & N-compound & $\begin{array}{l}\text { Biosynthesis of AA; Arg and pro metabolism, glutathione metabolism, } \\
\text { biosynthesis of alkaloids from ornithine }\end{array}$ & Extra \\
\hline Hydroxylamine & N-compound & Nitrogen metabolism & Extra \\
\hline Fructose & Sugar & $\begin{array}{l}\text { Fructose and mannose metabolism, galactose metabolism, starch and } \\
\text { sucrose metabolism }\end{array}$ & Extra \\
\hline Galactose & Sugar & Galactose metabolism & Extra \\
\hline Arabinose & Sugar & Pentose and glucuronate interconversion & Extra \\
\hline Lyxose & Sugar & Pentose and glucuronate interconversion & Extra \\
\hline Xylose & Sugar & Pentose and glucuronate interconversion & Extra \\
\hline Trehalose & Sugar & Starch and sucrose metabolism & Extra \\
\hline Glycerate & Sugar acid & PPP, gly, ser and thr metaboim, glyoxylate metabolism & Extra \\
\hline
\end{tabular}

*AA $=$ amino acid, $\mathrm{FA}=$ fatty acid, $\mathrm{PPP}=$ pentose phosphate pathway, TCA=tricarboxylic acid cycle 
Table S6: Identified intracellular metabolites detected during UV-A + PAR including chemical class and possible biosynthetic pathways (KEGG).

\begin{tabular}{|c|c|c|}
\hline Name & Class & Pathway \\
\hline Glyoxylate & Acid & Glyoxylate metabolism, AA metabolism \\
\hline 2-Oxobutanoate & Acid & Biosynthesis of AA; cys and met metabolism, thr metabolism. Val, leu and ile biosynthesis \\
\hline 4-Hydroxybutyric acid & Acid & Carbon fixation pathways in prokaryotes \\
\hline 5-Oxoproline & Acid & Glutathione metabolism \\
\hline Boric acid & Acid & Aminobenzoate degradation, microbial metabolism in diverse environments \\
\hline Hydroxyisovalerate & Acid & Val, leu and ile degradation \\
\hline Lactic acid & Acid & Glycolysis/fructose and mannose metabolism, pyruvate metabolism. \\
\hline Methylbenzoate & Acid & Degradation of aromatics \\
\hline Oxalate & Acid & Glyoxylate metabolism, biosynthesis of secondary metabolites \\
\hline Phosphoric acid & Acid & PS/oxidative phosphorylation \\
\hline Propan-2-ol & Alcohol & Propanoate metabolism \\
\hline 1-Butanol & Alcohol & Butanoate metabolism, microbial metabolism, degradation of aromatic compounds \\
\hline Ethane-1,2-diol & Alcohol & Glyoxylate and dicarboxylate metabolism \\
\hline Propane-1,3-diol & Alcohol & Glycerolipid metabolism \\
\hline Glutamate & Amino acid & $\begin{array}{l}\text { Biosynthesis of AA; arg, ala, asp and glu, arg and pro metabolism. Forms L-ornithine/L- } \\
\text { glutamine }\end{array}$ \\
\hline Leucine & Amino acid & $\begin{array}{l}\text { Biosynthesis of AA; gly, ser, val, leu and ile metabolism, biosynthesis of } \\
\text { alkaloids/secondary metabolites }\end{array}$ \\
\hline Phenylalanine & Amino acid & $\begin{array}{l}\text { Biosynthesis of AA. Phe metabolism via shikimate pathway, Tyr metabolism, biosynthesis } \\
\text { of alkaloids derived from shikimate pathway and ornithine. }\end{array}$ \\
\hline Serine & Amino acid & $\begin{array}{l}\text { Biosynthesis of AA; gly, ser and thr metabolism, glyoxylate metabolism and biosynthesis of } \\
\text { secondary metabolites. Chl biosynthesis, MAA biosynthesis. }\end{array}$ \\
\hline Histamine & Aromatic & Biosynthesis of alkaloids derived from histidine and pyrine, secondary metabolites \\
\hline Lumichrome & Aromatic & Riboflavin metabolism \\
\hline
\end{tabular}




\begin{tabular}{lll}
\hline Name & Class & Pathway \\
\hline Eicosanoic acid & Fatty acid & FA biosynthesis, biosynthesis of unsaturated FA \\
Mystiric acid & Fatty acid & FA biosynthesis, biosynthesis of unsaturated FA \\
Palmitic acid & Fatty acid & FA biosynthesis, biosynthesis of unsaturated FA \\
Stearic acid & Fatty acid & FA biosynthesis, biosynthesis of unsaturated FA \\
Ethanolamine & N-compound & Glycerophosphlipid metabolism, pyruvate metabolism \\
Cyanamide & Other & Atrazine degradation \\
Hydrogen sulfide & S-compound & Sulfur metabolism, microbial metabolism in diverse environments \\
Mannose & Sugar & Fructose and mannose metabolism, amino sugar and nucleotide metabolism, galactose \\
Sucrose & Sugar & metabolism \\
$*$ AA = amino acid, FA = fatty acid, PPP = pentose phosphate pathway, TCA=tricarboxylic acid cycle
\end{tabular}

*AA $=$ amino acid, $\mathrm{FA}=$ fatty acid, $\mathrm{PPP}=$ pentose phosphate pathway, $\mathrm{TCA}=$ tricarboxylic acid cycle 
Table S7: Identified intracellular metabolites detected during acclimation \& shock and shock only UV-A + PAR experiments including chemical class and possible biosynthetic pathways (KEGG).

\begin{tabular}{|c|c|c|c|}
\hline Name & Class & Pathway & Acc\&Shock/Shock \\
\hline Methylbenzoate & Acid & Degradation of aromatics & Acc\&Shock \\
\hline Boric acid & Acid & $\begin{array}{l}\text { Aminobenzoate degradation, microbial metabolism in diverse } \\
\text { environments }\end{array}$ & Acc\&Shock \\
\hline $\begin{array}{l}\text { 3-Hydroxy-L-1-pyrroline-5- } \\
\text { carboxylate }\end{array}$ & Acid & Arg and pro metabolism & Acc\&Shock \\
\hline Lactic acid & Acid & Glycolysis/fructose and mannose metabolism, pyruvate metabolism. & Acc\&Shock \\
\hline Phosphoric acid & Acid & PS/oxidative phosphorylation & Acc\&Shock \\
\hline Malate & Acid & $\begin{array}{l}\text { TCA, Pyruvate metabolism, glyoxylate metabolism, biosynthesis of } \\
\text { secondary metabolites }\end{array}$ & Acc\&Shock \\
\hline Phytol & Alcohol & Chl metabolism & Acc\&Shock \\
\hline Glutamate & Amino acid & $\begin{array}{l}\text { Biosynthesis of AA; arg, ala, asp and glu, arg and pro metabolism. } \\
\text { Forms L-ornithine/L-glutamine }\end{array}$ & Acc\&Shock \\
\hline Valine & Amino acid & $\begin{array}{l}\text { Biosynthesis of AA; val, leu and ile metabolism, biosynthesis of } \\
\text { alkaloids/secondary metabolites }\end{array}$ & Acc\&Shock \\
\hline Proline & Amino acid & $\begin{array}{l}\text { Biosynthesis of AA; Arg and pro biosynthesis, secondary metabolite } \\
\text { biosynthesis }\end{array}$ & Acc\&Shock \\
\hline Cyanuric acid & Aromatic & Atrazine degredation & Acc\&Shock \\
\hline $\begin{array}{l}\text { 1-Hydroxy-3-methoxy-6- } \\
\text { methylanthraquinone }\end{array}$ & Aromatic & Polyketide synthesis & Acc\&Shock \\
\hline $\begin{array}{l}\text { 4-Hydroxyanthraquinone-2- } \\
\text { carboxylic acid }\end{array}$ & Aromatic & Polyketide synthesis & Acc\&Shock \\
\hline Adenine & Aromatic & Purine metabolism & Acc\&Shock \\
\hline Lumichrome & Aromatic & Riboflavin metabolism & Acc\&Shock \\
\hline Mystiric acid & Fatty acid & FA biosynthesis, biosynthesis of unsaturated FA & Acc\&Shock \\
\hline Palmitic acid & Fatty acid & FA biosynthesis, biosynthesis of unsaturated FA & Acc\&Shock \\
\hline
\end{tabular}




\begin{tabular}{lllc}
\hline Name & Class & Pathway & Acc\&Shock/Shock \\
\hline Stearic acid & Fatty acid & FA biosynthesis, biosynthesis of unsaturated FA & Acc\&Shock \\
Ethanolamine & N-compound & Glycerophosphlipid metabolism, pyruvate metabolism & Acc\&Shock \\
Cyanamide & Other & Atrazine degradation & Acc\&Shock \\
Hydrogen sulfide & S-compound & Sulfur metabolism, microbial metabolism in diverse environments & Acc\&Shock \\
Fructose & Sugar & Fructose and mannose metabolism, galactose metabolism, starch and & Acc\&Shock \\
Arabinose & Sucrose metabolism & Acc\&Shock \\
Raffinose & Sugar & Pentose and glucuronate interconversion & Acc\&Shock \\
Sucrose & Sugar & Galactose metabolism & Acc\&Shock \\
Trehalose & Sugar & Starch and sucrose metabolism & Acc\&Shock \\
Glycerol 3-phosphate & Sugar & Glycerolipid/glycerophospholipid metabolism, metabolism of & Acc\&Shock \\
Methylbenzoate & phosphate & secondary metabolites & Shock \\
Boric acid & Acid & Degradation of aromatics & Shinobenzoate degradation, microbial metabolism in diverse \\
3-Hydroxy-L-1-pyrroline-5- & Acid & environments & Shock \\
carboxylate & Acid & Arg and pro metabolism & Shock \\
Lactic acid & Acid & Glycolysis/fructose and mannose metabolism, pyruvate metabolism. & Shock \\
Phosphoric acid & Acid & PS/oxidative phosphorylation & Shock \\
Fumarate & Acid & TCA, Pyruvate metabolism, glyoxylate metabolism, secondary & Shetabiltes \\
Phytol & Alcohol & Chl metabolism & Shock \\
Glycerol & Alcohol & Pentose and glucuronate interconversion & Shock \\
Glutamate & Amino acid & Biosynthesis of AA; arg, ala, asp and glu, arg and pro metabolism. & Forms L-ornithine/L-glutamine \\
Valine & Amino acid & Biosynthesis of AA; val, leu and ile metabolism, biosynthesis of & alkaloids/secondary metabolites \\
\hline
\end{tabular}




\begin{tabular}{|c|c|c|c|}
\hline Name & Class & Pathway & Acc\&Shock/Shock \\
\hline Aspartate & Amino acid & $\begin{array}{l}\text { Biosynthesis of AA; ala, asp and glu metabolism. Arg, gly, ser and } \\
\text { thr metabolism, cys and met metabolism. Carbon fixation }\end{array}$ & Shock \\
\hline Alanine & Amino acid & $\begin{array}{l}\text { Biosynthesis of AA; ala, asp and glu metabolism, cys and met } \\
\text { metabolism, carbon fixation and biosynthesis of secondary } \\
\text { metabolites }\end{array}$ & Shock \\
\hline Proline & Amino acid & $\begin{array}{l}\text { Biosynthesis of AA; Arg and pro biosynthesis, secondary metabolite } \\
\text { biosynthesis }\end{array}$ & Shock \\
\hline 5-Oxoproline & Amino acid & Glutathione metabolism & Shock \\
\hline $\begin{array}{l}\text { 1-Hydroxy-3-methoxy-6- } \\
\text { methylanthraquinone }\end{array}$ & Aromatic & Polyketide synthesis & Shock \\
\hline $\begin{array}{l}\text { 4-Hydroxyanthraquinone-2- } \\
\text { carboxylic acid }\end{array}$ & Aromatic & Polyketide synthesis & Shock \\
\hline Lumichrome & Aromatic & Riboflavin metabolism & Shock \\
\hline Mystiric acid & Fatty acid & FA biosynthesis, biosynthesis of unsaturated FA & Shock \\
\hline Palmitoleic acid & Fatty acid & FA biosynthesis, biosynthesis of unsaturated FA & Shock \\
\hline Palmitic acid & Fatty acid & FA biosynthesis, biosynthesis of unsaturated FA & Shock \\
\hline Oleic acid & Fatty acid & FA biosynthesis, biosynthesis of unsaturated FA & Shock \\
\hline Stearic acid & Fatty acid & FA biosynthesis, biosynthesis of unsaturated FA & Shock \\
\hline Ethanolamine & N-compound & Glycerophosphlipid metabolism, pyruvate metabolism & Shock \\
\hline Cyanamide & Other & Atrazine degradation & Shock \\
\hline Hydrogen sulfide & S-compound & Sulfur metabolism, microbial metabolism in diverse environments & Shock \\
\hline $\begin{array}{l}\text { Cholest-2-eno[2,3-b]indole, 1'- } \\
\text { acetyl-6'-methoxy- }\end{array}$ & Sterol & Sterol synthesis & Shock \\
\hline Fructose & Sugar & $\begin{array}{l}\text { Fructose and mannose metabolism, galactose metabolism, starch and } \\
\text { sucrose metabolism }\end{array}$ & Shock \\
\hline Galactose & Sugar & Galactose metabolism & Shock \\
\hline Arabinose & Sugar & Pentose and glucuronate interconversion & Shock \\
\hline
\end{tabular}




\begin{tabular}{lllc}
\hline Name & Class & Pathway & Acc\&Shock/Shock \\
\hline Raffinose & Sugar & Galactose metabolism & Shock \\
Sucrose & Sugar & Galactose metabolism, starch and sucrose metabolism & Shock \\
Trehalose & Sugar & Starch and sucrose metabolism & Shock \\
\multirow{2}{*}{ Glycerol 3-phosphate } & Sugar & Glycerolipid/glycerophospholipid metabolism, metabolism of & Shock \\
\hline \multirow{2}{*}{ *AA = amino acid, FA = fatty acid, PPP = pentose phosphate pathway, TCA=tricarboxylic acid cycle } & secondary metabolites
\end{tabular}


Table S8: One-way ANOVA results showing metabolites with overall significance $(p<0.05)$ including Tukey's Honestly Significant difference (HSD) post-hoc test results of significant pairs of solvent extracts. Data was normalised by sum, log transformed and mean centred prior to analysis. Missing data points were assumed to be lower than detection limit and replaced with half of the minimum integrated signal for statistical analysis.

\begin{tabular}{|c|c|c|c|c|c|}
\hline \multirow[b]{2}{*}{ Metabolite } & \multicolumn{2}{|l|}{ ANOVA } & \multicolumn{2}{|c|}{ Tukey's HSD } & \multirow[b]{2}{*}{ Tukey's HSD 4} \\
\hline & $p$-value & Tukey's HSD 1 & Tukey's HSD 2 & Tukey's HSD 3 & \\
\hline Valine & $2.17 \mathrm{E}-13$ & MeOH vs Hex & $\mathrm{MeOH}$ vs EtOAc & $\mathrm{MeOH}$ vs EtOH & \\
\hline Glycerol & $1.35 \mathrm{E}-07$ & $\mathrm{MeOH}$ vs Hex & $\mathrm{MeOH}$ vs EtOAc & $\mathrm{MeOH}$ vs EtOH & \\
\hline Threonic acid & $2.42 \mathrm{E}-07$ & $\mathrm{MeOH}$ vs Hex & $\mathrm{MeOH}$ vs EtOAc & $\mathrm{MeOH}$ vs $\mathrm{EtOH}$ & \\
\hline Glycerate & $5.01 \mathrm{E}-07$ & $\mathrm{MeOH}$ vs Hex & $\mathrm{MeOH}$ vs EtOAc & $\mathrm{MeOH}$ vs $\mathrm{EtOH}$ & \\
\hline Putrescine & $9.54 \mathrm{E}-07$ & $\mathrm{MeOH}$ vs Hex & $\mathrm{MeOH}$ vs EtOAc & $\mathrm{MeOH}$ vs $\mathrm{EtOH}$ & \\
\hline Ribitol & $1.18 \mathrm{E}-06$ & $\mathrm{MeOH}$ vs Hex & $\mathrm{MeOH}$ vs EtOAc & $\mathrm{MeOH}$ vs $\mathrm{EtOH}$ & \\
\hline Serine & 4.79E-06 & $\mathrm{MeOH}$ vs Hex & $\mathrm{MeOH}$ vs EtOAc & $\mathrm{MeOH}$ vs EtOH & \\
\hline Pregnenolone & 0.000209 & EtOH vs Hex & $\mathrm{MeOH}$ vs Hex & EtOH vs EtOAc & $\mathrm{MeOH}$ vs EtOAc \\
\hline Sucrose & 0.000232 & EtOAc vs Hex & $\mathrm{MeOH}$ vs Hex & EtOH vs EtOAc & $\mathrm{MeOH}$ vs EtOAc \\
\hline Hydroxylamine & 0.000365 & EtOAc vs Hex & $\mathrm{MeOH}$ vs Hex & EtOH vs EtOAc & $\mathrm{MeOH}$ vs EtOH \\
\hline Propane-1,2-diol & 0.000927 & EtOH vs Hex & EtOH vs EtOAc & $\mathrm{MeOH}$ vs EtOH & \\
\hline 3-Methylbenzaldehyde & 0.001185 & $\mathrm{MeOH}$ vs Hex & $\mathrm{MeOH}$ vs EtOAc & $\mathrm{MeOH}$ vs $\mathrm{EtOH}$ & \\
\hline Capric acid & 0.001594 & $\mathrm{MeOH}$ vs Hex & $\mathrm{MeOH}$ vs EtOAc & $\mathrm{MeOH}$ vs EtOH & \\
\hline Mystric acid & 0.002547 & $\mathrm{MeOH}$ vs Hex & $\mathrm{MeOH}$ vs EtOAc & $\mathrm{MeOH}$ vs EtOH & \\
\hline Lauric acid & 0.007996 & EtOAc vs Hex & $\mathrm{MeOH}$ vs EtOAc & & \\
\hline Methylbenzoate & 0.016976 & $\mathrm{MeOH}$ vs Hex & $\mathrm{MeOH}$ vs EtOH & & \\
\hline
\end{tabular}




\section{References}

[1] Lau, N.-S.; Matsui, M.; Abdullah, A. A. Cyanobacteria : Photoautotrophic Microbial Factories for the Sustainable Synthesis of Industrial Products. Biomed Res. Int. 2015, 2015. https://doi.org/10.1155/2015/754934.

[2] Latifi, A.; Ruiz, M.; Zhang, C. C. Oxidative Stress in Cyanobacteria. FEMS Microbiol. Rev. 2009, 33 (2), 258-278. https://doi.org/10.1111/j.15746976.2008.00134.x.

[3] Field, C. B. Primary Production of the Biosphere: Integrating Terrestrial and Oceanic Components. Science (80-. ). 1998, 281 (5374), 237-240. https://doi.org/10.1126/science.281.5374.237.

[4] Ramanan, R.; Kim, B. H.; Cho, D. H.; Oh, H. M.; Kim, H. S. Algae-Bacteria Interactions: Evolution, Ecology and Emerging Applications. Biotechnol. Adv. 2016, 34 (1), 14-29. https://doi.org/10.1016/j.biotechadv.2015.12.003.

[5] Stanier, R. Y.; Deruelles, J.; Rippka, R.; Herdman, M.; Waterbury, J. B. Generic Assignments, Strain Histories and Properties of Pure Cultures of Cyanobacteria. Microbiology 1979, 111 (1), 1-61. https://doi.org/10.1099/00221287-111-1-1.

[6] Kultschar, B.; Llewellyn, C. Secondary Metabolites in Cyanobacteria. In Secondary Metabolites - Sources and Applications; InTech, 2018; Vol. 2, p 64. https://doi.org/10.5772/intechopen.75648.

[7] Palinska, K. a.; Surosz, W. Taxonomy of Cyanobacteria: A Contribution to Consensus Approach. Hydrobiologia 2014, 740 (1), 1-11. https://doi.org/10.1007/s10750-014-1971-9.

[8] Komárek, J.; Kaštovský, J.; Mareš, J.; Johansen, J. R. Taxonomic Classification of Cyanoprokaryotes (Cyanobacterial Genera) 2014, Using a Polyphasic Approach. Preslia 2014, 86 (4), 295-335.

[9] Seckbach, J. Algae and Cyanobacteria in Extreme Environments, Illustrate; Seckbach, J., Ed.; Cellular Origin, Life in Extreme Habitats and Astrobiology; Springer Netherlands: Dordrecht, 2007; Vol. 11. https://doi.org/10.1007/9781-4020-6112-7.

[10] Hartmann, A.; Albert, A.; Ganzera, M. Effects of Elevated Ultraviolet Radiation on Primary Metabolites in Selected Alpine Algae and Cyanobacteria. J. Photochem. Photobiol. B Biol. 2015, 149, 149-155. https://doi.org/10.1016/j.jphotobiol.2015.05.016.

[11] Chrismas, N. A. M.; Anesio, A. M.; Sánchez-Baracaldo, P. Multiple Adaptations to Polar and Alpine Environments within Cyanobacteria: A Phylogenomic and Bayesian Approach. Front. Microbiol. 2015, 6 (OCT), 110. https://doi.org/10.3389/fmicb.2015.01070.

[12] Berman-Frank, I.; Lundgren, P.; Falkowski, P. Nitrogen Fixation and Photosynthetic Oxygen Evolution in Cyanobacteria. Res. Microbiol. 2003, 154 
(3), 157-164. https://doi.org/10.1016/S0923-2508(03)00029-9.

[13] Burja, A. M.; Banaigs, B.; Abou-Mansour, E.; Grant Burgess, J.; Wright, P. C. Marine Cyanobacteria - a Prolific Source of Natural Products. Tetrahedron 2001, 57 (46), 9347-9377. https://doi.org/10.1016/S0040-4020(01)00931-0.

[14] Burja, A. M.; Dhamwichukorn, S.; Wright, P. C. Cyanobacterial Postgenomic Research and Systems Biology. Trends Biotechnol. 2003, 21 (11), 504-511. https://doi.org/10.1016/j.tibtech.2003.08.008.

[15] Kehr, J. C.; Picchi, D. G.; Dittmann, E. Natural Product Biosyntheses in Cyanobacteria: A Treasure Trove of Unique Enzymes. Beilstein Journal of Organic Chemistry. 2011, pp 1622-1635. https://doi.org/10.3762/bjoc.7.191.

[16] Abed, R. M. M.; Dobretsov, S.; Sudesh, K. Applications of Cyanobacteria in Biotechnology. J. Appl. Microbiol. 2009, 106 (1), 1-12. https://doi.org/10.1111/j.1365-2672.2008.03918.x.

[17] S. Rai, S. Pandey, A. K. Shrivastava, P. K. Singh, C. A. and L. C. R. Understanding of Abiotic Stress Tolerance in Cyanobacterium Using Proteomics. In Stress Biology of Cyanobacteria; 2015; pp 93-107.

[18] Sakamoto, T.; Yoshida, T.; Arima, H.; Hatanaka, Y.; Takani, Y.; Tamaru, Y. Accumulation of Trehalose in Response to Desiccation and Salt Stress in the Terrestrial Cyanobacterium Nostoc Commune. Phycol. Res. 2009, 57 (1), 6673. https://doi.org/10.1111/j.1440-1835.2008.00522.x.

[19] Surosz, W.; Palinska, K. A. Effects of Heavy-Metal Stress on Cyanobacterium Anabaena Flos-Aquae. Arch. Environ. Contam. Toxicol. 2004, 48 (1), 40-48. https://doi.org/10.1007/s00244-004-0163-4.

[20] Portwich, A.; Garcia-Pichel, F. Ultraviolet and Osmotic Stresses Induce and Regulate the Synthesis of Mycosporines in the Cyanobacterium Chlorogloeopsis PCC 6912. Arch. Microbiol. 1999, 172 (4), 187-192. https://doi.org/10.1007/s002030050759.

[21] Rastogi, R. P.; Sinha, R. P. Biotechnological and Industrial Significance of Cyanobacterial Secondary Metabolites. Biotechnol. Adv. 2009, 27 (4), 521539. https://doi.org/10.1016/j.biotechadv.2009.04.009.

[22] Singh, R.; Parihar, P.; Singh, M.; Bajguz, A.; Kumar, J.; Singh, S.; Singh, V. P.; Prasad, S. M. Uncovering Potential Applications of Cyanobacteria and Algal Metabolites in Biology, Agriculture and Medicine: Current Status and Future Prospects. Front. Microbiol. 2017, 8 (APR), 1-37. https://doi.org/10.3389/fmicb.2017.00515.

[23] Sinha, R. P.; Häder, D. P. UV-Protectants in Cyanobacteria. Plant Sci. 2008, 174 (3), 278-289. https://doi.org/10.1016/j.plantsci.2007.12.004.

[24] Rastogi, R. P.; Sinha, R. P.; Moh, S. H.; Lee, T. K.; Kottuparambil, S.; Kim, Y. J.; Rhee, J. S.; Choi, E. M.; Brown, M. T.; Häder, D. P.; et al. Ultraviolet Radiation and Cyanobacteria. J. Photochem. Photobiol. B Biol. 2014, 141, 154-169. https://doi.org/10.1016/j.jphotobiol.2014.09.020. 
[25] Rastogi, R. P. UV-Induced Oxidative Stress in Cyanobacteria: How Life Is Able to Survive? Biochem. Anal. Biochem. 2015, 04 (02), 2-5. https://doi.org/10.4172/2161-1009.1000173.

[26] Richa; Sinha, R. P. Biochemical Characterization of Sunscreening Mycosporine-like Amino Acids from Two Nostoc Species Inhabiting Diverse Habitats. Protoplasma 2014, 252 (1), 199-208. https://doi.org/10.1007/s00709-014-0674-4.

[27] Moon, Y.-J.; Kim, S. Il; Chung, Y.-H. Sensing and Responding to UV-A in Cyanobacteria. Int. J. Mol. Sci. 2012, 13 (12), 16303-16332. https://doi.org/10.3390/ijms131216303.

[28] Wada, N.; Sakamoto, T.; Matsugo, S. Multiple Roles of Photosynthetic and Sunscreen Pigments in Cyanobacteria Focusing on the Oxidative Stress. Metabolites 2013, 3 (2), 463-483. https://doi.org/10.3390/metabo3020463.

[29] Sinha, R. P.; Singh, S. P.; Häder, D.-P. Database on Mycosporines and Mycosporine-like Amino Acids (MAAs) in Fungi, Cyanobacteria, Macroalgae, Phytoplankton and Animals. J. Photochem. Photobiol. B Biol. 2007, 89 (1), 29-35. https://doi.org/10.1016/j.jphotobiol.2007.07.006.

[30] Gupta, V.; Ratha, S. K.; Sood, A.; Chaudhary, V.; Prasanna, R. New Insights into the Biodiversity and Applications of Cyanobacteria (Blue-Green Algae)—Prospects and Challenges. Algal Res. 2013, 2 (2), 79-97. https://doi.org/10.1016/j.algal.2013.01.006.

[31] Derikvand, P.; Llewellyn, C. a.; Purton, S. Cyanobacterial Metabolites as a Source of Sunscreens and Moisturizers: A Comparison with Current Synthetic Compounds. Eur. J. Phycol. 2017, 52 (1), 43-56. https://doi.org/10.1080/09670262.2016.1214882.

[32] Rastogi, R. P.; Sonani, R. R.; Madamwar, D.; Incharoensakdi, A. Characterization and Antioxidant Functions of Mycosporine-like Amino Acids in the Cyanobacterium Nostoc Sp. R76DM. Algal Res. 2016, 16, 110-118. https://doi.org/10.1016/j.algal.2016.03.009.

[33] Carreto, J. I.; Carignan, M. O.; Montoya, N. G. A High-Resolution ReversePhase Liquid Chromatography Method for the Analysis of Mycosporine-like Amino Acids (MAAs) in Marine Organisms. Mar. Biol. 2005, 146 (2), 237252. https://doi.org/10.1007/s00227-004-1447-y.

[34] Nazifi, E.; Wada, N.; Asano, T.; Nishiuchi, T.; Iwamuro, Y.; Chinaka, S.; Matsugo, S.; Sakamoto, T. Characterization of the Chemical Diversity of Glycosylated Mycosporine-like Amino Acids in the Terrestrial Cyanobacterium Nostoc Commune. J. Photochem. Photobiol. B. 2014, 142C, 154-168. https://doi.org/10.1016/j.jphotobiol.2014.12.008.

[35] Wang, Y.; Liu, S.; Hu, Y.; Li, P.; Wan, J. B. Current State of the Art of Mass Spectrometry-Based Metabolomics Studies - a Review Focusing on Wide Coverage, High Throughput and Easy Identification. RSC Adv. 2015, 5 (96), 78728-78737. https://doi.org/10.1039/c5ra14058g. 
[36] Fiehn, O. Metabolomics - The Link between Genotypes and Phenotypes. Plant Mol. Biol. 2002, 48 (1-2), 155-171.

https://doi.org/10.1023/A:1013713905833.

[37] Armitage, E. G.; Barbas, C. Metabolomics in Cancer Biomarker Discovery: Current Trends and Future Perspectives. J. Pharm. Biomed. Anal. 2014, 87, 111. https://doi.org/10.1016/j.jpba.2013.08.041.

[38] Rhee, E. P.; Gerszten, R. E. Metabolomics and Cardiovascular Biomarker Discovery. Clin. Chem. 2012, 58 (1), 139-147. https://doi.org/10.1373/clinchem.2011.169573.

[39] Kuhlisch, C.; Pohnert, G. Metabolomics in Chemical Ecology. Nat. Prod. Rep. 2015, 32 (7), 937-955. https://doi.org/10.1039/c5np00003c.

[40] Okazaki, Y.; Saito, K. Recent Advances of Metabolomics in Plant Biotechnology. Plant Biotechnol. Rep. 2012, 6 (1), 1-15. https://doi.org/10.1007/s11816-011-0191-2.

[41] Tang, J. Microbial Metabolomics. Curr. Genomics 2011, 12 (6), 391-403. https://doi.org/10.2174/138920211797248619.

[42] Dettmer, K.; Aronov, P. A.; Hammock, B. D. Mass Spectrometry-Based Metabolomics. Mass Spectrom. Rev. 2007, 26 (1), 51-78. https://doi.org/10.1002/mas.20108.

[43] Schwarz, D.; Orf, I.; Kopka, J.; Hagemann, M. Recent Applications of Metabolomics Toward Cyanobacteria. Metabolites 2013, 3 (4), 72-100. https://doi.org/10.3390/metabo3010072.

[44] Krall, L.; Huege, J.; Catchpole, G.; Steinhauser, D.; Willmitzer, L. Assessment of Sampling Strategies for Gas Chromatography-mass Spectrometry (GC-MS) Based Metabolomics of Cyanobacteria. $J$. Chromatogr. B 2009, 877 (27), 2952-2960. https://doi.org/10.1016/j.jchromb.2009.07.006.

[45] Veyel, D.; Erban, A.; Fehrle, I.; Kopka, J.; Schroda, M. Rationales and Approaches for Studying Metabolism in Eukaryotic Microalgae. Metabolites 2014, 4 (2), 184-217. https://doi.org/10.3390/metabo4020184.

[46] Bolten, C. J.; Kiefer, P.; Letisse, F.; Portais, J.-C.; Wittmann, C. Sampling for Metabolome Analysis of Microorganisms. Anal. Chem. 2007, 79 (10), 38433849. https://doi.org/10.1021/ac0623888.

[47] Mushtaq, M. Y.; Choi, Y. H.; Verpoorte, R.; Wilson, E. G. Extraction for Metabolomics: Access to The Metabolome. Phytochem. Anal. 2014, 25 (4), 291-306. https://doi.org/10.1002/pca.2505.

[48] Portwich, A.; Garcia-Pichel, F. A Novel Prokaryotic UVB Photoreceptor in the Cyanobacterium Chlorogloeopsis PCC 6912. Photochem. Photobiol. 2000, 71 (4), 493-498. https://doi.org/10.1562/00318655(2000)0710493ANPUPI2.0.CO2. 
[49] Rastogi, R. P.; Incharoensakdi, A. Characterization of UV-Screening Compounds, Mycosporine-like Amino Acids, and Scytonemin in the Cyanobacterium Lyngbya Sp. CU2555. FEMS Microbiol. Ecol. 2014, 87 (1), 244-256. https://doi.org/10.1111/1574-6941.12220.

[50] Joshi, D.; Mohandass, C.; Dhale, M. Effect of UV-B Radiation and Desiccation Stress on Photoprotective Compounds Accumulation in Marine Leptolyngbya Sp. Appl. Biochem. Biotechnol. 2017, 1-13. https://doi.org/10.1007/s12010-017-2523-3.

[51] Airs, R. L.; Temperton, B.; Sambles, C.; Farnham, G.; Skill, S. C.; Llewellyn, C. a. Chlorophyll $\mathrm{f}$ and Chlorophyll $\mathrm{d}$ Are Produced in the Cyanobacterium Chlorogloeopsis Fritschii When Cultured under Natural Light and NearInfrared Radiation. FEBS Lett. 2014, 588 (20), 3770-3777. https://doi.org/10.1016/j.febslet.2014.08.026.

[52] Wase, N.; Pham, T. K.; Ow, S. Y.; Wright, P. C. Quantitative Analysis of UVA Shock and Short Term Stress Using ITRAQ, Pseudo Selective Reaction Monitoring (PSRM) and GC-MS Based Metabolite Analysis of the Cyanobacterium Nostoc Punctiforme ATCC 29133. J. Proteomics 2014, 109, 332-355. https://doi.org/10.1016/j.jprot.2014.06.024.

[53] Eisenhut, M.; Huege, J.; Schwarz, D.; Bauwe, H.; Kopka, J.; Hagemann, M. Metabolome Phenotyping of Inorganic Carbon Limitation in Cells of the Wild Type and Photorespiratory Mutants of the Cyanobacterium Synechocystis Sp. Strain PCC 6803 . Plant Physiol. 2008, 148 (4), 2109-2120. https://doi.org/10.1104/pp.108.129403.

[54] Fiore, C. L.; Longnecker, K.; Kido Soule, M. C.; Kujawinski, E. B. Release of Ecologically Relevant Metabolites by the Cyanobacterium S Ynechococcus Elongatus CCMP 1631. Environ. Microbiol. 2015, 17 (10), 3949-3963. https://doi.org/10.1111/1462-2920.12899.

[55] Vilhauer, L.; Jervis, J.; Ray, W. K.; Helm, R. F. The Exo-Proteome and ExoMetabolome of Nostoc Punctiforme (Cyanobacteria) in the Presence and Absence of Nitrate. Arch. Microbiol. 2014, 196 (5), 357-367. https://doi.org/10.1007/s00203-014-0974-2.

[56] Markley, J. L.; Brüschweiler, R.; Edison, A. S.; Eghbalnia, H. R.; Powers, R.; Raftery, D.; Wishart, D. S. The Future of NMR-Based Metabolomics. Curr. Opin. Biotechnol. 2017, 43, 34-40. https://doi.org/10.1016/j.copbio.2016.08.001.

[57] Fan, T. W.-M.; Lane, A. N. Applications of NMR Spectroscopy to Systems Biochemistry. Prog. Nucl. Magn. Reson. Spectrosc. 2016, 92-93, 18-53. https://doi.org/10.1016/j.pnmrs.2016.01.005.

[58] Ren, J. L.; Zhang, A. H.; Kong, L.; Wang, X. J. Advances in Mass Spectrometry-Based Metabolomics for Investigation of Metabolites. RSC Adv. 2018, 8 (40), 22335-22350. https://doi.org/10.1039/c8ra01574k.

[59] Fiehn, O. Metabolomics by Gas Chromatography-Mass Spectrometry: 
Combined Targeted and Untargeted Profiling. In Current Protocols in Molecular Biology; John Wiley \& Sons, Inc.: Hoboken, NJ, USA, 2016; Vol. 131 A, p 30.4.1-30.4.32. https://doi.org/10.1002/0471142727.mb3004s114.

[60] Halket, J. M.; Waterman, D.; Przyborowska, A. M.; Patel, R. K. P.; Fraser, P. D.; Bramley, P. M. Chemical Derivatization and Mass Spectral Libraries in Metabolic Profiling by GC/MS and LC/MS/MS. J. Exp. Bot. 2005, 56 (410), 219-243. https://doi.org/10.1093/jxb/eri069.

[61] Schauer, N.; Steinhauser, D.; Strelkov, S.; Schomburg, D.; Allison, G.; Moritz, T.; Lundgren, K.; Roessner-Tunali, U.; Forbes, M. G.; Willmitzer, L.; et al. GC-MS Libraries for the Rapid Identification of Metabolites in Complex Biological Samples. FEBS Lett. 2005, 579 (6), 1332-1337. https://doi.org/10.1016/j.febslet.2005.01.029.

[62] Kopka, J.; Schauer, N.; Krueger, S.; Birkemeyer, C.; Usadel, B.; Bergmüller, E.; Dörmann, P.; Weckwerth, W.; Gibon, Y.; Stitt, M.; et al. GMD@CSB.DB: The Golm Metabolome Database. Bioinformatics 2005, 21 (8), 1635-1638. https://doi.org/10.1093/bioinformatics/bti236.

[63] Sumner, L. W.; Amberg, A.; Barrett, D.; Beale, M. H.; Beger, R.; Daykin, C. A.; Fan, T. W. M.; Fiehn, O.; Goodacre, R.; Griffin, J. L.; et al. Proposed Minimum Reporting Standards for Chemical Analysis. Metabolomics 2007, 3 (3), 211-221. https://doi.org/10.1007/s11306-007-0082-2.

[64] Ehling-Schulz, M.; Bilger, W.; Scherer, S. UV-B-Induced Synthesis of Photoprotective Pigments and Extracellular Polysaccharides in the Terrestrial Cyanobacterium Nostoc Commune. J. Bacteriol. 1997, 179 (6), 1940-1945. https://doi.org/10.1128/jb.179.6.1940-1945.1997.

[65] Singh, S. P.; Klisch, M.; Sinha, R. P.; Hader, D.-P. Effects of Abiotic Stressors on Synthesis of the Mycosporine-like Amino Acid Shinorine in the Cyanobacterium Anabaena Variabilis PCC 7937. Photochem. Photobiol. 2008, 84, 1500-1505. https://doi.org/10.1111/j.1751-1097.2008.00376.x.

[66] Dai, Y. J.; Li, J.; Wei, S. M.; Chen, N.; Xiao, Y. P.; Tan, Z. L.; Jia, S. R.; Yuan, N. N.; Tan, N.; Song, Y. J. Effect of Light with Different Wavelengths on Nostoc Flagelliforme Cells in Liquid Culture. J. Microbiol. Biotechnol. 2013, 23, 534-538. https://doi.org/10.4014/jmb.1205.05037.

[67] Dempo, Y.; Ohta, E.; Nakayama, Y.; Bamba, T.; Fukusaki, E. Molar-Based Targeted Metabolic Profiling of Cyanobacterial Strains with Potential for Biological Production. Metabolites 2014, 4 (2), 499-516. https://doi.org/10.3390/metabo4020499.

[68] Sue, T.; Obolonkin, V.; Griffiths, H.; Villas-Bôas, S. G. An Exometabolomics Approach to Monitoring Microbial Contamination in Microalgal Fermentation Processes by Using Metabolic Footprint Analysis. Appl. Environ. Microbiol. 2011, 77 (21), 7605-7610. https://doi.org/10.1128/AEM.00469-11.

[69] Pinu, F.; Villas-Boas, S. Extracellular Microbial Metabolomics: The State of the Art. Metabolites 2017, 7 (3), 43. https://doi.org/10.3390/metabo7030043. 
[70] Granucci, N.; Pinu, F. R.; Han, T.-L.; Villas-Boas, S. G. Can We Predict the Intracellular Metabolic State of a Cell Based on Extracellular Metabolite Data? Mol. Biosyst. 2015, 11 (12), 3297-3304. https://doi.org/10.1039/C5MB00292C.

[71] Wijffels, R. H.; Kruse, O.; Hellingwerf, K. J. Potential of Industrial Biotechnology with Cyanobacteria and Eukaryotic Microalgae. Current Opinion in Biotechnology. June 2013, pp 405-413. https://doi.org/10.1016/j.copbio.2013.04.004.

[72] Rosgaard, L.; de Porcellinis, A. J.; Jacobsen, J. H.; Frigaard, N. U.; Sakuragi, Y. Bioengineering of Carbon Fixation, Biofuels, and Biochemicals in Cyanobacteria and Plants. J. Biotechnol. 2012, 162 (1), 134-147. https://doi.org/10.1016/j.jbiotec.2012.05.006.

[73] Angermayr, S. A.; Hellingwerf, K. J.; Lindblad, P.; Teixeira de, M. J. Energy Biotechnology with Cyanobacteria. Curr. Opin. Biotechnol. 2009, 20 (3), 257-263. https://doi.org/10.1016/j.copbio.2009.05.011.

[74] Spolaore, P.; Joannis-Cassan, C.; Duran, E.; Isambert, A. Commercial Applications of Microalgae. J. Biosci. Bioeng. 2006, 101 (2), 87-96.

[75] Evans, E. H.; Foulds, I.; Carr, N. G. Environmental Conditions and Morphological Variation in the Blue-Green Alga Chlorogloea Fritschii. $J$. Gen. Microbiol. 1976, 92 (1), 147-155. https://doi.org/10.1099/00221287-921-147.

[76] Balasundaram, B.; Skill, S. C.; Llewellyn, C. a. A Low Energy Process for the Recovery of Bioproducts from Cyanobacteria Using a Ball Mill. Biochem. Eng. J. 2012, 69, 48-56. https://doi.org/10.1016/j.bej.2012.08.010.

[77] Chaiklahan, R.; Chirasuwan, N.; Loha, V.; Bunnag, B. Lipid and Fatty Acids Extraction from the Cyanobacterium Spirulina. ScienceAsia 2008, 34 (3), 299. https://doi.org/10.2306/scienceasia1513-1874.2008.34.299.

[78] Rastogi, R. P.; Madamwar, D.; Incharoensakdi, A. Sun-Screening Bioactive Compounds Mycosporine-like Amino Acids in Naturally Occurring Cyanobacterial Biofilms: Role in Photoprotection. J. Appl. Microbiol. 2015, 119 (3), 753-762. https://doi.org/10.1111/jam.12879.

[79] Rastogi, R. P.; Sonani, R. R.; Madamwar, D. Cyanobacterial Sunscreen Scytonemin: Role in Photoprotection and Biomedical Research. Appl. Biochem. Biotechnol. 2015, 176 (39), 1551-1563. https://doi.org/10.1007/s12010-015-1676-1.

[80] Krzemińska, I.; Pawlik-Skowrońska, B.; Trzcińska, M.; Tys, J. Influence of Photoperiods on the Growth Rate and Biomass Productivity of Green Microalgae. Bioprocess Biosyst. Eng. 2014, 37 (4), 735-741. https://doi.org/10.1007/s00449-013-1044-x.

[81] Styczynski, M. P.; Moxley, J. F.; Tong, L. V.; Walther, J. L.; Jensen, K. L.; Stephanopoulos, G. N. Systematic Identification of Conserved Metabolites in 
GC/MS Data for Metabolomics and Biomarker Discovery. Anal. Chem. 2007, 79 (3), 966-973. https://doi.org/10.1021/ac0614846.

[82] Chong, J.; Soufan, O.; Li, C.; Caraus, I.; Li, S.; Bourque, G.; Wishart, D. S.; Xia, J. MetaboAnalyst 4.0: Towards More Transparent and Integrative Metabolomics Analysis. Nucleic Acids Res. 2018, 46 (W1), W486-W494. https://doi.org/10.1093/nar/gky310.

[83] Xia, J.; Sinelnikov, I. V.; Wishart, D. S. MetATT: A Web-Based Metabolomics Tool for Analyzing Time-Series and Two-Factor Datasets. Bioinformatics 2011, 27 (17), 2455-2456. https://doi.org/10.1093/bioinformatics/btr392.

[84] Kanehisa, M. KEGG: Kyoto Encyclopedia of Genes and Genomes. Nucleic Acids Res. 2000, 28 (1), 27-30. https://doi.org/10.1093/nar/28.1.27.

[85] Ritchie, R. J. Consistent Sets of Spectrophotometric Chlorophyll Equations for Acetone, Methanol and Ethanol Solvents. Photosynth. Res. 2006, 89 (1), $27-$ 41. https://doi.org/10.1007/s11120-006-9065-9.

[86] Henriques, M.; Silva, a; Rocha, J. Extraction and Quantification of Pigments from a Marine Microalga: A Simple and Reproducible Method. Commun. Curr. Res. Educ. Top. Trends Appl. Microbiol. 2007, 586-593.

[87] O.H Lowry, N.J. Rosebrough, A. L. F. and R. J. R. Protein Measurement with the Folin Phenol Reagent. J. Biol. Chem. 1951, 193, 265-275.

[88] Slocombe, S. P.; Ross, M.; Thomas, N.; Mcneill, S.; Stanley, M. S. Bioresource Technology A Rapid and General Method for Measurement of Protein in Micro-Algal Biomass. Bioresour. Technol. 2013, 129, 51-57. https://doi.org/10.1016/j.biortech.2012.10.163.

[89] DuBois, M.; Gilles, K. a.; Hamilton, J. K.; Rebers, P. a.; Smith, F. Colorimetric Method for Determination of Sugars and Related Substances. Anal. Chem. 1956, 28 (3), 350-356. https://doi.org/10.1021/ac60111a017.

[90] Masuko, T.; Minami, A.; Iwasaki, N.; Majima, T.; Nishimura, S.-I.; Lee, Y. C. Carbohydrate Analysis by a Phenol-sulfuric Acid Method in Microplate Format. Anal. Biochem. 2005, 339 (1), 69-72. https://doi.org/10.1016/j.ab.2004.12.001.

[91] Cevallos-Cevallos, J. M.; Jines, C.; Maridueña-Zavala, M. G.; MolinaMiranda, M. J.; Ochoa, D. E.; Flores-Cedeno, J. A. GC-MS Metabolite Profiling for Specific Detection of Dwarf Somaclonal Variation in Banana Plants. Appl. Plant Sci. 2018, 6 (11), 1-9. https://doi.org/10.1002/aps3.1194.

[92] Rao, G.; Sui, J.; Zhang, J. Metabolomics Reveals Significant Variations in Metabolites and Correlations Regarding the Maturation of Walnuts ( Juglans Regia L.). Biol. Open 2016, 5 (6), 829-836. https://doi.org/10.1242/bio.017863.

[93] Willamme, R.; Alsafra, Z.; Arumugam, R.; Eppe, G.; Remacle, F.; Levine, R. D.; Remacle, C. Metabolomic Analysis of the Green Microalga 
Chlamydomonas Reinhardtii Cultivated under Day/Night Conditions. $J$. Biotechnol. 2015, 215, 20-26. https://doi.org/10.1016/j.jbiotec.2015.04.013.

[94] Vidoudez, C.; Pohnert, G. Comparative Metabolomics of the Diatom Skeletonema Marinoi in Different Growth Phases. Metabolomics 2011, 8 (4), 654-669. https://doi.org/10.1007/s11306-011-0356-6.

[95] Obata, T.; Schoenefeld, S.; Krahnert, I.; Bergmann, S.; Scheffel, A.; Fernie, A. R. Gas-Chromatography Mass-Spectrometry (GC-MS) Based Metabolite Profiling Reveals Mannitol as a Major Storage Carbohydrate in the Coccolithophorid Alga Emiliania Huxleyi. Metabolites 2013, 3 (1), 168-184. https://doi.org/10.3390/metabo3010168.

[96] Kusano, M.; Tohge, T.; Fukushima, A.; Kobayashi, M.; Hayashi, N.; Otsuki, H.; Kondou, Y.; Goto, H.; Kawashima, M.; Matsuda, F.; et al. Metabolomics Reveals Comprehensive Reprogramming Involving Two Independent Metabolic Responses of Arabidopsis to UV-B Light. Plant J. 2011, 67 (2), 354-369. https://doi.org/10.1111/j.1365-313X.2011.04599.x.

[97] Pascual, J.; Cañal, M. J.; Escandón, M.; Meijón, M.; Weckwerth, W.; Valledor, L. Integrated Physiological, Proteomic, and Metabolomic Analysis of Ultra Violet (UV) Stress Responses and Adaptation Mechanisms in Pinus Radiata. Mol. Cell. Proteomics 2017, 16 (3), 485-501. https://doi.org/10.1074/mcp.M116.059436.

[98] Bölling, C.; Fiehn, O. Metabolite Profiling of Chlamydomonas Reinhardtii under Nutrient Deprivation. Plant Physiol. 2005, 139 (December), 1995-2005. https://doi.org/10.1104/pp.105.071589.2.

[99] Shen, S. G.; Jia, S. R.; Yan, R. R.; Wu, Y. K.; Wang, H. Y.; Lin, Y. H.; Zhao, D. X.; Tan, Z. L.; Lv, H. X.; Han, P. P. The Physiological Responses of Terrestrial Cyanobacterium: Nostoc Flagelliforme to Different Intensities of Ultraviolet-B Radiation. RSC Adv. 2018, 8 (38), 21065-21074. https://doi.org/10.1039/c8ra04024a.

[100] Tambellini, N.; Zaremberg, V.; Turner, R.; Weljie, A. Evaluation of Extraction Protocols for Simultaneous Polar and Non-Polar Yeast Metabolite Analysis Using Multivariate Projection Methods. Metabolites 2013, 3 (3), 592-605. https://doi.org/10.3390/metabo3030592.

[101] Winder, C. L.; Dunn, W. B.; Schuler, S.; Broadhurst, D.; Jarvis, R.; Stephens, G. M.; Goodacre, R. Global Metabolic Profiling of Escherichia Coli Cultures: An Evaluation of Methods for Quenching and Extraction of Intracellular Metabolites. Anal. Chem. 2008, 80 (8), 2939-2948. https://doi.org/10.1021/ac7023409.

[102] Armstrong, R. A. When to Use the Bonferroni Correction. Ophthalmic Physiol. Opt. 2014, 34 (5), 502-508. https://doi.org/10.1111/opo.12131.

[103] Gromski, P.; Xu, Y.; Kotze, H.; Correa, E.; Ellis, D.; Armitage, E.; Turner, M.; Goodacre, R. Influence of Missing Values Substitutes on Multivariate Analysis of Metabolomics Data. Metabolites 2014, 4 (2), 433-452. 
https://doi.org/10.3390/metabo4020433.

[104] Padmaperuma, G.; Kapoore, R. V.; Gilmour, D. J.; Vaidyanathan, S. Microbial Consortia: A Critical Look at Microalgae Co-Cultures for Enhanced Biomanufacturing. Crit. Rev. Biotechnol. 2018, 38 (5), 690-703. https://doi.org/10.1080/07388551.2017.1390728.

[105] Navarro Llorens, J. M.; Tormo, A.; Martínez-García, E. Stationary Phase in Gram-Negative Bacteria. FEMS Microbiol. Rev. 2010, 34 (4), 476-495. https://doi.org/10.1111/j.1574-6976.2010.00213.x.

[106] Lu, S.; Wang, J.; Niu, Y.; Yang, J.; Zhou, J.; Yuan, Y. Metabolic Profiling Reveals Growth Related FAME Productivity and Quality of Chlorella Sorokiniana with Different Inoculum Sizes. Biotechnol. Bioeng. 2012, 109 (7), 1651-1662. https://doi.org/10.1002/bit.24447.

[107] Zech, H.; Thole, S.; Schreiber, K.; Kalhöfer, D.; Voget, S.; Brinkhoff, T.; Simon, M.; Schomburg, D.; Rabus, R. Growth Phase-Dependent Global Protein and Metabolite Profiles of Phaeobacter Gallaeciensis Strain DSM 17395, a Member of the Marine Roseobacter-Clade. Proteomics 2009, 9 (14), 3677-3697. https://doi.org/10.1002/pmic.200900120.

[108] Watanabe, S.; Ohbayashi, R.; Kanesaki, Y.; Saito, N.; Chibazakura, T.; Soga, T.; Yoshikawa, H. Intensive DNA Replication and Metabolism during the Lag Phase in Cyanobacteria. PLoS One 2015, 10 (9), 1-16. https://doi.org/10.1371/journal.pone.0136800.

[109] Widhalm, J. R.; Dudareva, N. A Familiar Ring to It: Biosynthesis of Plant Benzoic Acids. Mol. Plant 2015, 8 (1), 83-97. https://doi.org/10.1016/j.molp.2014.12.001.

[110] Jang, Y. S.; Kim, B.; Shin, J. H.; Choi, Y. J.; Choi, S.; Song, C. W.; Lee, J.; Park, H. G.; Lee, S. Y. Bio-Based Production of C2-C6 Platform Chemicals. Biotechnol. Bioeng. 2012, 109 (10), 2437-2459. https://doi.org/10.1002/bit.24599.

[111] Buchweitz, L. F.; Yurkovich, J. T.; Blessing, C.; Kohler, V.; Schwarzkopf, F.; King, Z. a; Yang, L.; Johannsson, F.; Sigurjonsson, O.; Rolfsson, O.; et al. Visualizing Metabolic Network Dynamics through Time-Series Metabolomics Data. bioRxiv 2018, 426106. https://doi.org/10.1101/426106.

[112] Marangoni, R.; Paris, D.; Melck, D.; Fulgentini, L.; Colombetti, G.; Motta, A. In Vivo NMR Metabolic Profiling of Fabrea Salina Reveals Sequential Defense Mechanisms against Ultraviolet Radiation. Biophys. J. 2011, 100 (1), 215-224. https://doi.org/10.1016/j.bpj.2010.10.050.

[113] Ehling-Schulz, M.; Schulz, S.; Wait, R.; Görg, A.; Scherer, S. The UV-B Stimulon of the Terrestrial Cyanobacterium Nostoc Commune Comprises Early Shock Proteins and Late Acclimation Proteins. Mol. Microbiol. 2002, 46 (3), 827-843. https://doi.org/10.1046/j.1365-2958.2002.03209.x.

[114] Shrivastava, A. K.; Chatterjee, A.; Yadav, S.; Singh, P. K.; Singh, S.; Rai, L. 
C. UV-B Stress Induced Metabolic Rearrangements Explored with

Comparative Proteomics in Three Anabaena Species tr. J. Proteomics 2015, 127, 122-133. https://doi.org/10.1016/j.jprot.2015.05.014.

[115] Stuart, R. K.; Mayali, X.; Lee, J. Z.; Craig Everroad, R.; Hwang, M.; Bebout, B. M.; Weber, P. K.; Pett-Ridge, J.; Thelen, M. P. Cyanobacterial Reuse of Extracellular Organic Carbon in Microbial Mats. ISME J. 2016, 10 (5), 12401251. https://doi.org/10.1038/ismej.2015.180.

[116] Kujawinski, E. B. The Impact of Microbial Metabolism on Marine Dissolved Organic Matter. Ann. Rev. Mar. Sci. 2011, 3 (1), 567-599. https://doi.org/10.1146/annurev-marine-120308-081003.

[117] Yadav, S.; Sinha, R. P.; Tyagi, M. B.; Kumar, A. Cyanobacterial Secondary Metabolites. Int. J. Pharma Bio Sci. 2011, 2 (2), 144-167.

[118] Mota, R.; Guimarães, R.; Büttel, Z.; Rossi, F.; Colica, G.; Silva, C. J.; Santos, C.; Gales, L.; Zille, A.; De Philippis, R.; et al. Production and Characterization of Extracellular Carbohydrate Polymer from Cyanothece Sp. CCY 0110.

Carbohydr. Polym. 2013, 92 (2), 1408-1415.

https://doi.org/10.1016/j.carbpol.2012.10.070.

[119] Singh, S. P.; Häder, D. P.; Sinha, R. P. Cyanobacteria and Ultraviolet Radiation (UVR) Stress: Mitigation Strategies. Ageing Res. Rev. 2010, 9 (2), 79-90. https://doi.org/10.1016/j.arr.2009.05.004.

[120] Kumar, A.; Sinha, R. P.; Häder, D.-P. Effect of UV-B on Enzymes of Nitrogen Metabolism in the Cyanobacterium Nostoc Calcicola. J. Plant Physiol. 1996, 148 (1-2), 86-91. https://doi.org/10.1016/S01761617(96)80298-7.

[121] Cameron, J. C.; Pakrasi, H. B. Essential Role of Glutathione in Acclimation to Environmental and Redox Perturbations in the Cyanobacterium Synechocystis Sp. PCC 6803. Plant Physiol. 2010, 154 (4), 1672-1685. https://doi.org/10.1104/pp.110.162990.

[122] Liang, X.; Zhang, L.; Natarajan, S. K.; Becker, D. F. Proline Mechanisms of Stress Survival. Antioxid. Redox Signal. 2013, 19 (9), 998-1011. https://doi.org/10.1089/ars.2012.5074.

[123] Verbruggen, N.; Hermans, C. Proline Accumulation in Plants: A Review. Amino Acids 2008, 35 (4), 753-759. https://doi.org/10.1007/s00726-008-00616.

[124] Chris, A.; Zeeshan, M.; Abraham, G.; Prasad, S. M. Proline Accumulation in Cylindrospermum Sp. Environ. Exp. Bot. 2006, 57 (1-2), 154-159. https://doi.org/10.1016/j.envexpbot.2005.05.008.

[125] Pinu, F. R.; Granucci, N.; Daniell, J.; Han, T.-L.; Carneiro, S.; Rocha, I.; Nielsen, J.; Villas-Boas, S. G. Metabolite Secretion in Microorganisms: The Theory of Metabolic Overflow Put to the Test. Metabolomics 2018, 14 (4), 43. https://doi.org/10.1007/s11306-018-1339-7. 
[126] Ehling-Schulz, M.; Scherer, S. UV Protection in Cyanobacteria. Eur. J. Phycol. 1999, 34 (4), 329-338.

https://doi.org/10.1080/09670269910001736392.

[127] Kolman, M.; Nishi, C.; Perez-Cenci, M.; Salerno, G. Sucrose in Cyanobacteria: From a Salt-Response Molecule to Play a Key Role in Nitrogen Fixation. Life 2015, 5 (1), 102-126.

https://doi.org/10.3390/life5010102.

[128] Chen, B.; Wan, C.; Mehmood, M. A.; Chang, J. S.; Bai, F.; Zhao, X. Manipulating Environmental Stresses and Stress Tolerance of Microalgae for Enhanced Production of Lipids and Value-Added Products-A Review. Bioresour. Technol. 2017, 244, 1198-1206. https://doi.org/10.1016/j.biortech.2017.05.170.

[129] Pade, N.; Hagemann, M. Salt Acclimation of Cyanobacteria and Their Application in Biotechnology. Life 2015, 5 (1), 25-49. https://doi.org/10.3390/life5010025.

[130] Muramatsu, M.; Hihara, Y. Acclimation to High-Light Conditions in Cyanobacteria: From Gene Expression to Physiological Responses. J. Plant Res. 2012, 125 (1), 11-39. https://doi.org/10.1007/s10265-011-0454-6.

[131] Ho, M. Y.; Gan, F.; Shen, G.; Zhao, C.; Bryant, D. A. Far-Red Light Photoacclimation (FaRLiP) in Synechococcus Sp. PCC 7335: I. Regulation of FaRLiP Gene Expression. Photosynth. Res. 2017, 131 (2), 173-186. https://doi.org/10.1007/s11120-016-0309-z.

[132] Kitchener, R. L.; Grunden, A. M. Methods for Enhancing Cyanobacterial Stress Tolerance to Enable Improved Production of Biofuels and Industrially Relevant Chemicals. Appl. Microbiol. Biotechnol. 2018, 102 (4), 1617-1628. https://doi.org/10.1007/s00253-018-8755-5.

[133] Coker, J. A. Extremophiles and Biotechnology: Current Uses and Prospects. F1000Research 2016, 5, 396. https://doi.org/10.12688/f1000research.7432.1.

[134] Su, H. Y.; Chou, H. H.; Chow, T. J.; Lee, T. M.; Chang, J. S.; Huang, W. L.; Chen, H. J. Improvement of Outdoor Culture Efficiency of Cyanobacteria by Over-Expression of Stress Tolerance Genes and Its Implication as BioRefinery Feedstock. Bioresour. Technol. 2017, 244, 1294-1303. https://doi.org/10.1016/j.biortech.2017.04.074.

[135] He, Y. Y.; Häder, D. P. UV-B-Induced Formation of Reactive Oxygen Species and Oxidative Damage of the Cyanobacterium Anabaena Sp.: Protective Effects of Ascorbic Acid and N-Acetyl-L-Cysteine. J. Photochem. Photobiol. B Biol. 2002, 66 (2), 115-124. https://doi.org/10.1016/S1011-1344(02)002312.

[136] Djanaguiraman, M.; Sheeba, J. A.; Shanker, A. K.; Devi, D. D.; Bangarusamy, U. Rice Can Acclimate to Lethal Level of Salinity by Pretreatment with Sublethal Level of Salinity through Osmotic Adjustment. Plant Soil 2006, 284 (1-2), 363-373. https://doi.org/10.1007/s11104-006-0043-y. 
[137] Mishra, Y.; Chaurasia, N.; Rai, L. C. Heat Pretreatment Alleviates UV-B Toxicity in the Cyanobacterium Anabaena Doliolum: A Proteomic Analysis of Cross Tolerance. Photochem. Photobiol. 2009, 85 (3), 824-833. https://doi.org/10.1111/j.1751-1097.2008.00469.x.

[138] Zakar, T.; Laczko-Dobos, H.; Toth, T. N.; Gombos, Z. Carotenoids Assist in Cyanobacterial Photosystem II Assembly and Function. Front. Plant Sci. 2016, 7 (March), 1-7. https://doi.org/10.3389/fpls.2016.00295.

[139] Huang, J. J. H.; Cheung, P. C. K. +UVA Treatment Increases the Degree of Unsaturation in Microalgal Fatty Acids and Total Carotenoid Content in Nitzschia Closterium (Bacillariophyceae) and Isochrysis Zhangjiangensis (Chrysophyceae). Food Chem. 2011, 129 (3), 783-791. https://doi.org/10.1016/j.foodchem.2011.05.021.

[140] Xiaoyuan, C.; Yang, Q.; Zhao, F.; Qin, S.; Yang, Y.; Shen, J.; Lin, H. Comparative Analysis of Fatty Acid Desaturases in Cyanobacterial Genomes. Comp. Funct. Genomics 2008, 2008. https://doi.org/10.1155/2008/284508.

[141] Lai, X. J.; Yang, R.; Luo, Q. J.; Chen, J. J.; Chen, H. M.; Yan, X. J. Glycerol3-Phosphate Metabolism Plays a Role in Stress Response in the Red Alga Pyropia Haitanensis. J. Phycol. 2015, 51 (2), 321-331. https://doi.org/10.1111/jpy.12276.

[142] Merchant, S. S.; Kropat, J.; Liu, B.; Shaw, J.; Warakanont, J. TAG, You're It! Chlamydomonas as a Reference Organism for Understanding Algal Triacylglycerol Accumulation. Curr. Opin. Biotechnol. 2012, 23 (3), 352-363. https://doi.org/10.1016/j.copbio.2011.12.001.

[143] González-Minero, F.; Bravo-Díaz, L. The Use of Plants in Skin-Care Products, Cosmetics and Fragrances: Past and Present. Cosmetics 2018, 5 (3), 50. https://doi.org/10.3390/cosmetics5030050.

[144] Che, C. T.; Zhang, H. Plant Natural Products for Human Health. Int. J. Mol. Sci. 2019, 20 (4), 2-5. https://doi.org/10.3390/ijms20040830.

[145] Berthon, J. Y.; Nachat-Kappes, R.; Bey, M.; Cadoret, J. P.; Renimel, I.; Filaire, E. Marine Algae as Attractive Source to Skin Care. Free Radic. Res. 2017, 51 (6), 555-567. https://doi.org/10.1080/10715762.2017.1355550.

[146] Sathasivam, R.; Radhakrishnan, R.; Hashem, A.; Abd_Allah, E. F. Microalgae Metabolites: A Rich Source for Food and Medicine. Saudi J. Biol. Sci. 2019, 26 (4), 709-722. https://doi.org/10.1016/j.sjbs.2017.11.003.

[147] Morone, J.; Alfeus, A.; Vasconcelos, V.; Martins, R. Revealing the Potential of Cyanobacteria in Cosmetics and Cosmeceuticals - A New Bioactive Approach. Algal Res. 2019, 41 (May), 101541. https://doi.org/10.1016/j.algal.2019.101541.

[148] Brausch, J. M.; Rand, G. M. A Review of Personal Care Products in the Aquatic Environment: Environmental Concentrations and Toxicity. Chemosphere 2011, 82 (11), 1518-1532. 
https://doi.org/10.1016/j.chemosphere.2010.11.018.

[149] Al-Haj, L.; Lui, Y.; Abed, R.; Gomaa, M.; Purton, S. Cyanobacteria as Chassis for Industrial Biotechnology: Progress and Prospects. Life 2016, 6 (4), 42. https://doi.org/10.3390/life6040042.

[150] Richmond, J. M.; Harris, J. E. Immunology and Skin in Health and Disease. Cold Spring Harb. Perspect. Med. 2014, 4 (12), 1-20. https://doi.org/10.1101/cshperspect.a015339.

[151] Rea, I. M.; Gibson, D. S.; McGilligan, V.; McNerlan, S. E.; Denis Alexander, H.; Ross, O. A. Age and Age-Related Diseases: Role of Inflammation Triggers and Cytokines. Front. Immunol. 2018, 9 (APR), 1-28. https://doi.org/10.3389/fimmu.2018.00586.

[152] Chen, Y.; Lyga, J. Brain-Skin Connection: Stress, Inflammation and Skin Aging. Inflamm. Allergy-Drug Targets 2014, 13 (3), 177-190. https://doi.org/10.2174/1871528113666140522104422.

[153] Rinnerthaler, M.; Bischof, J.; Streubel, M. K.; Trost, A.; Richter, K. Oxidative Stress in Aging Human Skin. Biomolecules 2015, 5 (2), 545-589. https://doi.org/10.3390/biom5020545.

[154] Zhang, S.; Duan, E. Fighting against Skin Aging: The Way from Bench to Bedside. Cell Transplant. 2018, 27 (5), 729-738.

https://doi.org/10.1177/0963689717725755.

[155] Ansel, J.; Perry, P.; Brown, J.; Damm, D.; Phan, T.; Hart, C.; Luger, T.; Hefeneider, S. Cytokine Modulation of Keratinocyte Cytokines. J. Invest. Dermatol. 1990, 94 (6), 101S-107S. https://doi.org/10.1111/15231747.ep12876053.

[156] Colombo, I.; Sangiovanni, E.; Maggio, R.; Mattozzi, C.; Zava, S.; Corbett, Y.; Fumagalli, M.; Carlino, C.; Corsetto, P. A.; Scaccabarozzi, D.; et al. HaCaT Cells as a Reliable in Vitro Differentiation Model to Dissect the Inflammatory/Repair Response of Human Keratinocytes. Mediators Inflamm. 2017, 2017. https://doi.org/10.1155/2017/7435621.

[157] Zampetti, a.; Mastrofrancesco, a.; Flori, E.; Maresca, V.; Picardo, M.; Amerio, P.; Feliciani, C. Proinflammatory Cytokine Production in HaCaT Cells Treated by Eosin: Implications for the Topical Treatment of Psoriasis. Int. J. Immunopathol. Pharmacol. 2009, 22 (4), 1067-1075. https://doi.org/10.1177/039463200902200423.

[158] Cherng, S. C.; Cheng, S. N.; Tarn, A.; Chou, T. C. Anti-Inflammatory Activity of c-Phycocyanin in Lipopolysaccharide-Stimulated RAW 264.7

Macrophages. Life Sci. 2007, 81 (19-20), 1431-1435. https://doi.org/10.1016/j.lfs.2007.09.009.

[159] Ku, C. S.; Pham, T. X.; Park, Y.; Kim, B.; Shin, M. S.; Kang, I.; Lee, J. Edible Blue-Green Algae Reduce the Production of pro-Inflammatory Cytokines by Inhibiting NF-KB Pathway in Macrophages and Splenocytes. Biochim. 
Biophys. Acta - Gen. Subj. 2013, 1830 (4), 2981-2988.

https://doi.org/10.1016/j.bbagen.2013.01.018.

[160] Hameed, M. S. a; Hassan, S. H.; Mohammed, R.; Gamal, R. Isolation and Characterization of Antimicrobial Active Compounds from the Cyanobacterium Nostoc Commune Vauch. J. Pure Appl. Microbiol. 2013, 7 (1), 109-116.

[161] Abdulkhaleq, L. A.; Assi, M. A.; Abdullah, R.; Zamri-Saad, M.; Taufiq-Yap, Y. H.; Hezmee, M. N. M. The Crucial Roles of Inflammatory Mediators in Inflammation: A Review. Vet. World 2018, 11 (5), 627-635. https://doi.org/10.14202/vetworld.2018.627-635.

[162] Richmond, J. M.; Harris, J. E. Immunology and Skin in Health and Disease. Cold Spring Harb Perspect Med 2014, 4 (12), a015339. https://doi.org/10.1101/cshperspect.a015339.

[163] Şener, L. T.; Albeniz, G.; Dinç, B.; Albeniz, I. ICELLigence Real-Time Cell Analysis System for Examining the Cytotoxicity of Drugs to Cancer Cell Lines. Exp. Ther. Med. 2017, 14 (3), 1866-1870. https://doi.org/10.3892/etm.2017.4781.

[164] Garcia, S. N.; Gutierrez, L.; McNulty, A. Real-Time Cellular Analysis as a Novel Approach for in Vitro Cytotoxicity Testing of Medical Device Extracts. J. Biomed. Mater. Res. - Part A 2013, 101 A (7), 2097-2106. https://doi.org/10.1002/jbm.a.34507.

[165] Denizot, F.; Lang, R. Rapid Colorimetric Assay for Cell Growth and Survival. J. Immunol. Methods 1986, 89 (2), 271-277. https://doi.org/10.1016/00221759(86)90368-6.

[166] Balouiri, M.; Sadiki, M.; Ibnsouda, S. K. Methods for in Vitro Evaluating Antimicrobial Activity: A Review. J. Pharm. Anal. 2016, 6 (2), 71-79. https://doi.org/10.1016/j.jpha.2015.11.005.

[167] Adnan, M.; Nazim Uddin Chy, M.; Mostafa Kamal, A. T. M.; Azad, M. O. K.; Paul, A.; Uddin, S. B.; Barlow, J. W.; Faruque, M. O.; Park, C. H.; Cho, D. H. Investigation of the Biological Activities and Characterization of Bioactive Constituents of Ophiorrhiza Rugosa Var. Prostrata (D.Don) \& Mondal Leaves through in Vivo, in Vitro, and in Silico Approaches. Molecules 2019. https://doi.org/10.3390/molecules24071367.

[168] Aparna, V.; Dileep, K. V.; Mandal, P. K.; Karthe, P.; Sadasivan, C.; Haridas, M. Anti-Inflammatory Property of n-Hexadecanoic Acid: Structural Evidence and Kinetic Assessment. Chem. Biol. Drug Des. 2012, 80 (3), 434-439. https://doi.org/10.1111/j.1747-0285.2012.01418.x.

[169] Bose, B.; Choudhury, H.; Tandon, P.; Kumaria, S. Studies on Secondary Metabolite Profiling, Anti-Inflammatory Potential, in Vitro Photoprotective and Skin-Aging Related Enzyme Inhibitory Activities of Malaxis Acuminata, a Threatened Orchid of Nutraceutical Importance. J. Photochem. Photobiol. B Biol. 2017, 173 (July), 686-695. 
https://doi.org/10.1016/j.jphotobiol.2017.07.010.

[170] Kabara, J. J.; Swieczkowski, D. M.; Conley, A. J.; Truant, J. P. Fatty Acids and Derivatives as Antimicrobial Agents. Antimicrob. Agents Chemother. 1972, 2 (1), 23-28. https://doi.org/10.1128/AAC.2.1.23.

[171] Islam, M. T.; Ali, E. S.; Uddin, S. J.; Shaw, S.; Islam, M. A.; Ahmed, M. I.; Chandra Shill, M.; Karmakar, U. K.; Yarla, N. S.; Khan, I. N.; et al. Phytol: A Review of Biomedical Activities. Food Chem. Toxicol. 2018, 121 (May), 8294. https://doi.org/10.1016/j.fct.2018.08.032.

[172] Pejin, B.; Savic, A.; Sokovic, M.; Glamoclija, J.; Ciric, A.; Nikolic, M.; Radotic, K.; Mojovic, M. Further in Vitro Evaluation of Antiradical and Antimicrobial Activities of Phytol. Nat. Prod. Res. 2014, 28 (6), 372-376. https://doi.org/10.1080/14786419.2013.869692.

[173] Singh, U.; Jialal, I. Anti-Inflammatory Effects of $\alpha$-Tocopherol. Ann. N. Y. Acad. Sci. 2004, 1031 (Cvd), 195-203. https://doi.org/10.1196/annals.1331.019.

[174] Digby, J. E.; Martinez, F.; Jefferson, A.; Ruparelia, N.; Chai, J.; Wamil, M.; Greaves, D. R.; Choudhury, R. P. Anti-Inflammatory Effects of Nicotinic Acid in Human Monocytes Are Mediated by GPR109A Dependent Mechanisms. Arterioscler. Thromb. Vasc. Biol. 2012, 32 (3), 669-676. https://doi.org/10.1161/ATVBAHA.111.241836.

[175] Lorencini, M.; Brohem, C. A.; Dieamant, G. C.; Zanchin, N. I. T.; Maibach, H. I. Active Ingredients against Human Epidermal Aging. Ageing Res. Rev. 2014, 15 (1), 100-115. https://doi.org/10.1016/j.arr.2014.03.002.

[176] Oyamada, C.; Kaneniwa, M.; Ebitani, K.; Murata, M.; Ishihara, K. Mycosporine-like Amino Acids Extracted from Scallop (Patinopecten Yessoensis) Ovaries: UV Protection and Growth Stimulation Activities on Human Cells. Mar. Biotechnol. 2008, 10 (2), 141-150. https://doi.org/10.1007/s10126-007-9043-Z.

[177] Suh, S. S.; Hwang, J.; Park, M.; Seo, H. H.; Kim, H. S.; Lee, J. H.; Moh, S. H.; Lee, T. K. Anti-Inflammation Activities of Mycosporine-like Amino Acids (MAAs) in Response to UV Radiation Suggest Potential Anti-Skin Aging Activity. Mar. Drugs 2014, 12 (10), 5174-5187. https://doi.org/10.3390/md12105174.

[178] Yahfoufi, N.; Alsadi, N.; Jambi, M.; Matar, C. The Immunomodulatory and Anti-Inflammatory Role of Polyphenols. Nutrients 2018, 10 (11), 1-23. https://doi.org/10.3390/nu10111618.

[179] Spolaore, P.; Joannis-Cassan, C.; Duran, E.; Isambert, A. Commercial Applications of Microalgae. J. Biosci. Bioeng. 2006, 101 (2), 87-96. https://doi.org/10.1263/jbb.101.87.

[180] Wijffels, R. H.; Kruse, O.; Hellingwerf, K. J. Potential of Industrial Biotechnology with Cyanobacteria and Eukaryotic Microalgae. Curr. Opin. 
Biotechnol. 2013, 24 (3), 405-413.

https://doi.org/10.1016/j.copbio.2013.04.004.

[181] Angermayr, S. A.; Gorchs Rovira, A.; Hellingwerf, K. J. Metabolic Engineering of Cyanobacteria for the Synthesis of Commodity Products.

Trends Biotechnol. 2015, 33 (6), 352-361.

https://doi.org/10.1016/j.tibtech.2015.03.009.

[182] Katoch, M.; Mazmouz, R.; Chau, R.; Pearson, L. a.; Pickford, R.; Neilan, B. a. Heterologous Production of Cyanobacterial Mycosporine-like Amino Acids Mycosporine-Ornithine and Mycosporine-Lysine in Escherichia Coli. Appl. Environ. Microbiol. 2016, 82 (20), 6167-6173. https://doi.org/10.1128/AEM.01632-16.

[183] Wu, H.; Gao, K.; Villafañe, V. E.; Watanabe, T.; Helbling, E. W. Effects of Solar UV Radiation on Morphology and Photosynthesis of Filamentous Cyanobacterium Arthrospira Platensis. Appl. Environ. Microbiol. 2005, 71 (9), 5004-5013. https://doi.org/10.1128/AEM.71.9.5004-5013.2005.

[184] Liu, L.; Pohnert, G.; Wei, D. Extracellular Metabolites from Industrial Microalgae and Their Biotechnological Potential. Mar. Drugs 2016, 14 (10), 1-19. https://doi.org/10.3390/md14100191. 